Supporting Information

for

\title{
Chemodivergent Synthesis of Oxazoles and Oxime Ethers Initiated by Selective C-N/C-O Formation of Oximes and Diazo Esters
}

\author{
Zhenjie Qi and Shaozhong Wang* \\ State Key Laboratory of Coordination Chemistry, Jiangsu Key Laboratory of Advanced Organic \\ Materials, School of Chemistry and Chemical Engineering, Nanjing University, 163 Xianlin \\ avenue, Nanjing 210023, China. \\ E-mail: wangsz@nju.edu.cn
}

\section{Contents}

1. General informations

SI-2

2. General procedure for the synthesis of oxazoles

SI-2

3. Characterization data of oxazoles $\mathbf{3 a - 3 z}$

SI-2

4. General procedure for the synthesis of oxime ethers

5. Characterization data of oxime ethers $4 \mathbf{a}-\mathbf{4 z}$

6. Gram scale reactions

7. Control experiment

8. X-Ray crystallographic data of compounds $\mathbf{3 b}$ and $\mathbf{4 b}$

9. References

10. Copies of NMR spectra

SI-30

11. 2D NMR spectra of $\mathbf{3 v}$ 


\section{Genernal informations}

${ }^{1} \mathrm{H}$ NMR were recorded at $400 \mathrm{MHz},{ }^{13} \mathrm{C}$ NMR spectra were recorded at $100 \mathrm{MHz}$. Spectra were recorded in a $\mathrm{CDCl}_{3}, \mathrm{DMSO}-d_{6}$ solution using the residual protonated solvent as the internal standard, $J$ values are given in hertz. IR spectra were recorded on Fourier Transform infrared spectrometer and listed in $\mathrm{cm}^{-1}$. High-resolution mass spectral analyses (HRMS) were performed on a Q-TOF-MS spectrometer. Flash column chromatography was performed over silica gel (300-400 mesh). The 12V2835 blue LED light strip $(1.5 \mathrm{~cm} \Phi, 200 \mathrm{~cm}$ in length, purchased from Lingke Lighting Enterprise Store (China) with spectral range of 435-445 nm). The crystallizing dish (15 $\mathrm{cm} \Phi ; 7.5 \mathrm{~cm} \mathrm{~h}$, borosilicate glass) Shanghai Leigu Instrument Co., Ltd.), and the distance from the light source to the irradiation vessel $7 \mathrm{~cm}$ approximately. All commercially available reagents were used without further purification. Oximes were prepared according to the literature procedures. ${ }^{1}$ Diazo esters were prepared according to the literature procedures. ${ }^{2-4}$

\section{General procedure for the synthesis of oxazoles (Procedure A)}

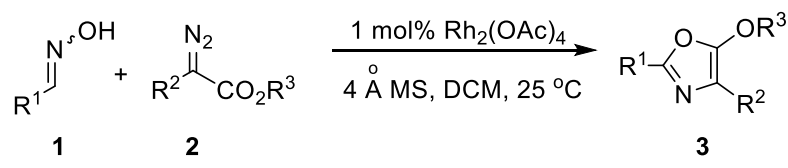

Under Ar atmosphere, to a $10 \mathrm{~mL}$ over-dried Schlenk tube charged with a stir bar, oxime $1(0.2$ mmol, 1.0 equiv.) and $\alpha$-diazoester 2 ( 0.4 mmol, 2.0 equiv.) and $4 \AA$ molecular sieves (20 mg), was added $\mathrm{Rh}_{2}(\mathrm{OAc})_{4}(0.002 \mathrm{mmol}, 1 \mathrm{~mol} \%)$. The resultant solution was stirred at $\mathrm{rt}$ for 8 hours (monitored by TLC). After completion, a saturated aqueous $\mathrm{NH}_{4} \mathrm{Cl}$ solution $(5 \mathrm{~mL})$ was added and the reaction mixture was diluted with ethyl acetate $(5 \mathrm{~mL})$. The organic layer was separated and the aqueous layer was extracted with EtOAc $(5 \mathrm{~mL} \times 3)$. The combined extracts were washed with brine $(10 \mathrm{~mL})$, dried over anhydrous $\mathrm{Na}_{2} \mathrm{SO}_{4}$. After filtration, the filtrate was concentrated under vacuum and the obtained residue was purified by flash column chromatography on silica gel (petroleum ether and ethyl acetate as eluents, the ratio is from 10:1 to 4:1) to afford oxazole 3 .

\section{Characterization data of oxazoles $3 a-3 z$}




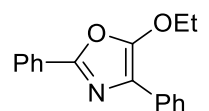

5-Ethoxy-2,4-diphenyloxazole (3a). According to procedure A, 3a was obtained from benzaldehyde oxime (24.2 mg, $0.2 \mathrm{mmol})$ and ethyl 2-diazo-2-phenylacetate (76.5 mg, $0.4 \mathrm{mmol})$ as light yellow oil $(37.1 \mathrm{mg}, 71 \%$ yield, eluent: petroleum ether/ethyl acetate $=20: 1) .{ }^{1} \mathrm{H}$ NMR $\left(400 \mathrm{MHz}, \mathrm{CDCl}_{3}\right) \delta$ 8.02-8.00 (m, 2H), 7.94-7.91 (m, 2H), 7.47-7.40 (m, 5H), 7.26-7.22 (m, $\left.1 \mathrm{H}\right)$, $4.47-4.42(\mathrm{q}, J=7.1 \mathrm{~Hz}, 2 \mathrm{H}), 1.53-1.50(\mathrm{t}, J=7.1 \mathrm{~Hz}, 3 \mathrm{H}) \mathrm{ppm} ;{ }^{13} \mathrm{C} \mathrm{NMR}\left(100 \mathrm{MHz}, \mathrm{CDCl}_{3}\right) \delta$ $153.9,152.0,131.5,129.7,128.7,128.5,127.7,126.5,125.6,125.1,116.9,69.8,15.2 \mathrm{ppm} ; \mathrm{IR}$ (Neat) $v_{\max } 3062,2982,2929,2108,1738,1632,1497,1243,1021,770,691 \mathrm{~cm}^{-1}$; HRMS (ESI) $m / z:[\mathrm{M}+\mathrm{H}]^{+}$Calcd for $\mathrm{C}_{17} \mathrm{H}_{16} \mathrm{NO}_{2} 266.1176$; Found 266.1175 .

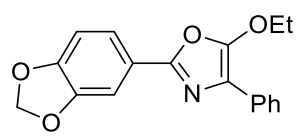

2-(Benzo $[d][1,3]$ dioxol-5-yl)-5-ethoxy-4-phenyloxazole (3b). According to procedure A, $3 \mathrm{~b}$ was obtained from benzo $[d][1,3]$ dioxole-5-carbaldehyde oxime $(33.1 \mathrm{mg}, 0.2 \mathrm{mmol})$ and ethyl 2-diazo-2-phenylacetate $(76.5 \mathrm{mg}, 0.4 \mathrm{mmol})$ as light yellow solid $(43.5 \mathrm{mg}, 70 \%$ yield, eluent: petroleum ether/ethyl acetate $=15: 1) .{ }^{1} \mathrm{H}$ NMR $\left(400 \mathrm{MHz}, \mathrm{CDCl}_{3}\right) \delta$ 7.91-7.89 $(\mathrm{dd}, J=8.4,1.2$ $\mathrm{Hz}, 2 \mathrm{H}), 7.55-7.52(\mathrm{dd}, J=8.2,1.7 \mathrm{~Hz}, 1 \mathrm{H}), 7.48-7.47$ (d, $J=1.6 \mathrm{~Hz}, 1 \mathrm{H}), 7.42-7.38(\mathrm{~m}, 2 \mathrm{H})$, 7.22-7.21 (m, 1H), 6.88-6.86 (d, $J=8.1 \mathrm{~Hz}, 1 \mathrm{H}), 6.02(\mathrm{~s}, 2 \mathrm{H}), 4.43-4.38(\mathrm{q}, J=7.1 \mathrm{~Hz}, 2 \mathrm{H})$, $1.52-1.48(\mathrm{t}, J=7.1 \mathrm{~Hz}, 3 \mathrm{H}) \mathrm{ppm} ;{ }^{13} \mathrm{C}$ NMR $\left(100 \mathrm{MHz}, \mathrm{CDCl}_{3}\right) \delta 153.6,151.9,149.0,148.0$, $131.5,128.4,126.4,125.1,121.9,120.2,116.8,108.5,106.1,101.5,69.9,15.2$ ppm; IR (Neat) $v_{\max } 2983,2900,2158,2024,1967,1734,1636,1502,1485,1381,1238,1201,1038,935,812$, $696 \mathrm{~cm}^{-1}$; HRMS (ESI) $\mathrm{m} / z$ : $[\mathrm{M}+\mathrm{H}]^{+}$Calcd for $\mathrm{C}_{18} \mathrm{H}_{16} \mathrm{NO}_{4} 310.1074$; Found 310.1074 .

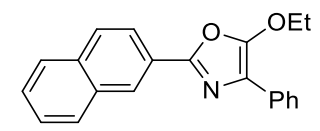

5-Ethoxy-2-(naphthalen-2-yl)-4-phenyloxazole (3c). According to procedure A, 3c was obtained from 2-naphthaldehyde oxime (34.3 mg, $0.2 \mathrm{mmol})$ and ethyl 2-diazo-2-phenylacetate $(76.2 \mathrm{mg}$, $0.4 \mathrm{mmol})$ as light white solid $(45.3 \mathrm{mg}, 72 \%$ yield, eluent: petroleum ether/ethyl acetate $=20: 1)$. ${ }^{1} \mathrm{H}$ NMR (400 MHz, DMSO- $\left.d_{6}\right) \delta 8.53(\mathrm{~d}, J=0.6 \mathrm{~Hz}, 1 \mathrm{H}), 8.12-8.08(\mathrm{~m}, 3 \mathrm{H}), 8.00-7.99(\mathrm{~m}, 1 \mathrm{H})$, 7.89-7.87 (m, 2H), 7.63-7.60 (m, 2H), 7.49-7.45 (m, 2H), 7.31-7.27 (m, 1H), 4.59-4.54 (q, $J=7.1$ 
$\mathrm{Hz}, 2 \mathrm{H}), 1.50-1.47(\mathrm{t}, J=7.1 \mathrm{~Hz}, 3 \mathrm{H}) \mathrm{ppm} ;{ }^{13} \mathrm{C}$ NMR (100 MHz, DMSO-d 6 ) $\delta 154.4,151.9$, 133.9, 133.2, 131.5, 129.3, 129.2, 129.0, 128.3, 127.8, 127.6, 127.1, 125.2, 125.0, 124.7, 122.9, 116.4, 70.6, 15.5 ppm; IR (Neat) $v_{\max } 3056,2982,2930,1734,1686,1632,1498,1381,1353$, 1197, 1019, 819, 750, 695, $474 \mathrm{~cm}^{-1}$; HRMS (ESI) $\mathrm{m} / z$ : $[\mathrm{M}+\mathrm{H}]^{+}$Calcd for $\mathrm{C}_{21} \mathrm{H}_{18} \mathrm{NO}_{2} 316.1332$; Found 316.1334 .

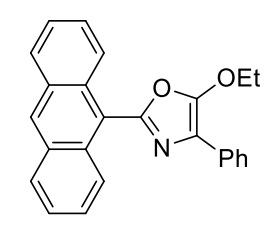

2-(Anthracen-9-yl)-5-ethoxy-4-phenyloxazole (3d). According to procedure A, 3d was obtained from anthracene-9-carbaldehyde oxime $(44.2 \mathrm{mg}, 0.2 \mathrm{mmol})$ and ethyl 2-diazo-2-phenylacetate (76.5 mg, $0.4 \mathrm{mmol}$ ) as light yellow solid $(46.9 \mathrm{mg}, 64 \%$ yield, eluent: petroleum ether/ethyl acetate $=20: 1) .{ }^{1} \mathrm{H}$ NMR $\left(400 \mathrm{MHz}, \mathrm{DMSO}-d_{6}\right) \delta 8.90(\mathrm{~s}, 1 \mathrm{H}), 8.24-8.22(\mathrm{~m}, 2 \mathrm{H}), 8.10-8.08(\mathrm{~m}$, 2H), 7.93-7.91 (m, 2H), 7.65-7.61 (m, 4H), 7.52-7.48 (m, 2H), 7.33-7.29 (m, 1H), 4.54-4.49 (q, $J$ $=7.1 \mathrm{~Hz}, 2 \mathrm{H}), 1.49-1.45(\mathrm{t}, J=7.1 \mathrm{~Hz}, 3 \mathrm{H}) \mathrm{ppm} ;{ }^{13} \mathrm{C} \mathrm{NMR}\left(100 \mathrm{MHz}, \mathrm{DMSO}-d_{6}\right) \delta 155.1,149.9$, 131.6, 131.2, 131.1, 130.7, 129.3, 129.2, 128.2, 127.1, 126.3, 125.6, 125.1, 121.4, 115.7, 70.5, 15.5 ppm; IR (Neat) $v_{\max } 3060,3053,2921,1750,1498,1454,1138,1263,1206,1178,1095$, 1046, 912, 732, $696 \mathrm{~cm}^{-1}$; HRMS (ESI) $\mathrm{m} / \mathrm{z}:[\mathrm{M}+\mathrm{H}]^{+}$Calcd for $\mathrm{C}_{25} \mathrm{H}_{20} \mathrm{NO}_{2}$ 366.1489; Found 366.1483 .

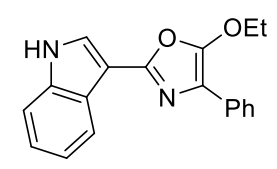

5-Ethoxy-2-(1H-indol-3-yl)-4-phenyloxazole (3e). According to procedure A, 3e was obtained from $1 H$-indole-3-carbaldehyde oxime $(32.3 \mathrm{mg}, 0.2 \mathrm{mmol})$ and ethyl 2-diazo-2-phenylacetate (76.5 mg, $0.4 \mathrm{mmol})$ as light yellow oil (26.3 mg, 42\% yield, eluent: petroleum ether/ethyl acetate $=5: 1) .{ }^{1} \mathrm{H}$ NMR $\left(400 \mathrm{MHz}, \mathrm{CDCl}_{3}\right) \delta 8.47$ (brs, $\left.1 \mathrm{H}\right), 8.40-8.36(\mathrm{~m}, 1 \mathrm{H}), 7.98-7.96(\mathrm{~m}, 2 \mathrm{H})$, 7.81-7.80 (d, $J=2.8 \mathrm{~Hz}, 1 \mathrm{H}), 7.44-7.40$ (m, 3H), 7.32-7.27 (m, 2H), 7.24-7.22 (m, 1H), 4.46-4.41 (q, $J=7.1 \mathrm{~Hz}, 2 \mathrm{H}), 1.54-1.51(\mathrm{t}, J=7.1 \mathrm{~Hz}, 3 \mathrm{H}) \mathrm{ppm} ;{ }^{13} \mathrm{C}$ NMR $\left(100 \mathrm{MHz}, \mathrm{CDCl}_{3}\right) \delta 152.7$, $150.6,136.1,131.9,128.5,126.2,125.1,124.7,124.3,123.2,121.4,121.3,116.1,111.4,106.2$, 70.0, 15.3 ppm; IR (Neat) $v_{\max } 3396,2981,2927,2157,1735,1643,1496,1448,1199,1018,746$, $696 \mathrm{~cm}^{-1}$; HRMS (ESI) $\mathrm{m} / z$ : $[\mathrm{M}+\mathrm{H}]^{+}$Calcd for $\mathrm{C}_{19} \mathrm{H}_{17} \mathrm{~N}_{2} \mathrm{O}_{2}$ 305.1285; Found 305.1287. 


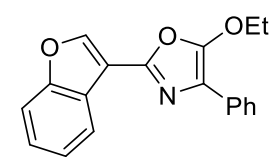

2-(Benzofuran-3-yl)-5-ethoxy-4-phenyloxazole (3f). According to procedure A, $3 \mathrm{f}$ was obtained from benzofuran-2-carbaldehyde oxime $(32.2 \mathrm{mg}, 0.2 \mathrm{mmol})$ and ethyl 2-diazo-2-phenylacetate (76.5 mg, $0.4 \mathrm{mmol}$ ) as light yellow oil (42.1 mg, 69\% yield, eluent: petroleum ether/ethyl acetate $=10: 1) .{ }^{1} \mathrm{H}$ NMR (400 MHz, $\left.\mathrm{CDCl}_{3}\right) \delta$ 7.96-7.94 (m, 2H), 7.65-7.60 (m, 2H), 7.44-7.40 (m, 2H), 7.39-7.35 (m, 1H), 7.31-7.24 (m, 3H), 4.51-4.45 (q, $J=7.2 \mathrm{~Hz}, 2 \mathrm{H}), 1.55-1.51(\mathrm{t}, J=7.2 \mathrm{~Hz}, 3 \mathrm{H})$ ppm; ${ }^{13} \mathrm{C}$ NMR (100 MHz, $\left.\mathrm{CDCl}_{3}\right) \delta 155.2,154.0,145.0,144.0,130.9,128.5,127.9,126.8,125.8$, 125.2, 123.6, 121.7, 117.3, 111.8, 106.1, 70.1, 15.2 ppm; IR (Neat) $v_{\max } 3060,2983,2925,1626$, 1497, 1446, 1382, 1353, 1310, 1258, 1172, 1076, 1018, 882, 749, $695 \mathrm{~cm}^{-1}$; HRMS (ESI) $\mathrm{m} / \mathrm{z}$ : $[\mathrm{M}+\mathrm{H}]^{+}$Calcd for $\mathrm{C}_{19} \mathrm{H}_{16} \mathrm{NO}_{3} 306.1125$; Found 306.1127 .

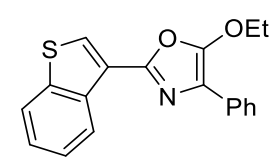

2-(Benzo[b]thiophen-3-yl)-5-ethoxy-4-phenyloxazole (3g). According to procedure A, 3g was obtained from benzo[b]thiophene-3-carbaldehyde oxime (35.5 $\mathrm{mg}, 0.2 \mathrm{mmol})$ and ethyl 2-diazo-2-phenylacetate $(76.5 \mathrm{mg}, 0.4 \mathrm{mmol})$ as light yellow oil $(41.2 \mathrm{mg}, 65 \%$ yield, eluent: petroleum ether/ethyl acetate $=20: 1) .{ }^{1} \mathrm{H}$ NMR $\left(400 \mathrm{MHz} \mathrm{CDCl}_{3}\right) \delta 8.94-8.92(\mathrm{~d}, J=8.1 \mathrm{~Hz}, 1 \mathrm{H})$, 8.01-7.98 (m, 3H), 7.91-7.89 (d, $J=8.1 \mathrm{~Hz}, 1 \mathrm{H})$, 7.56-7.52 (m, 1H), 7.46-7.42 (m, 3H), 7.28-7.24 $(\mathrm{m}, 1 \mathrm{H}), 4.49-4.44(\mathrm{q}, J=7.1 \mathrm{~Hz}, 2 \mathrm{H}), 1.55-1.51(\mathrm{t}, J=7.1 \mathrm{~Hz}, 3 \mathrm{H}) \mathrm{ppm} ;{ }^{13} \mathrm{C}$ NMR $(100 \mathrm{MHz}$, $\left.\mathrm{CDCl}_{3}\right) \delta 153.3,149.0,140.1,135.9,131.6,128.5,126.6,126.4,125.1,125.1,125.1,124.9,123.8$, 122.5, 116.3, 69.9, 15.2 ppm; IR (Neat) $v_{\max }$ 3102, 3061, 2981, 2929, 1735, 1631, 1603, 1497, 1376, 1352, 1260, 1199, 1018, 992, 839, 756, 735, 694, $514 \mathrm{~cm}^{-1}$; HRMS (ESI) $\mathrm{m} / z:[\mathrm{M}+\mathrm{H}]^{+}$ Calcd for $\mathrm{C}_{19} \mathrm{H}_{16} \mathrm{NO}_{2} \mathrm{~S}$ 322.0896; Found 322.0897.

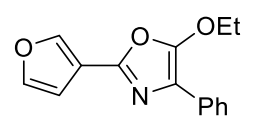

5-Ethoxy-2-(furan-3-yl)-4-phenyloxazole (3h). According to the procedure A, $3 \mathbf{h}$ was obtained from furan-2-carbaldehyde oxime $(22.2 \mathrm{mg}, 0.2 \mathrm{mmol})$ and ethyl 2-diazo-2-phenylacetate $(76.5$ $\mathrm{mg}, 0.4 \mathrm{mmol})$ as light yellow oil $(23.4 \mathrm{mg}, 45 \%$ yield, eluent: petroleum ether/ethyl acetate $=$ 15:1). ${ }^{1} \mathrm{H}$ NMR $\left(400 \mathrm{MHz}, \mathrm{CDCl}_{3}\right) \delta$ 7.92-7.89 (m, 2H), 7.55-7.54 (dd, $\left.J=1.7,0.7 \mathrm{~Hz}, 1 \mathrm{H}\right)$, 
7.42-7.38 (m, 2H), 7.26-7.22 (m, 1H), 6.94-6.93 (dd, $J=3.4,0.7 \mathrm{~Hz}, 1 \mathrm{H}), 6.54-6.52$ (dd, $J=3.4$, $1.8 \mathrm{~Hz}, 1 \mathrm{H}), 4.45-4.39(\mathrm{q}, J=7.1 \mathrm{~Hz}, 2 \mathrm{H}), 1.52-1.48(\mathrm{t}, J=7.1 \mathrm{~Hz}, 3 \mathrm{H}) \mathrm{ppm} ;{ }^{13} \mathrm{C}$ NMR $(100$ $\left.\mathrm{MHz}, \mathrm{CDCl}_{3}\right) \delta 153.4,145.3,143.9,142.9,131.0,128.4,126.6,125.2,116.7,111.7,110.1,70.1$, 15.2 ppm; IR (Neat) $v_{\max } 3355,3129,2983,2930,1736,1685,1633,1498,1382,1354,1314$, 1201, 1178, 1089, 1014, 884, 765, $696 \mathrm{~cm}^{-1}$; HRMS (ESI) $\mathrm{m} / z:[\mathrm{M}+\mathrm{H}]^{+}$Calcd for $\mathrm{C}_{15} \mathrm{H}_{14} \mathrm{NO}_{3}$ 256.0968; Found 256.0968.

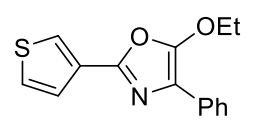

5-Ethoxy-4-phenyl-2-(thiophen-3-yl)oxazole (3i). According to procedure A, 3i was obtained from thiophene-2-carbaldehyde oxime $(25.4 \mathrm{mg}, 0.2 \mathrm{mmol})$ and ethyl 2-diazo-2-phenylacetate (76.5 mg, $0.4 \mathrm{mmol}$ ) as light yellow oil (17.2 mg, 32\% yield, eluent: petroleum ether/ethyl acetate $=20: 1) .{ }^{1} \mathrm{H}$ NMR $\left(400 \mathrm{MHz}, \mathrm{CDCl}_{3}\right) \delta 7.90-7.88(\mathrm{~m}, 2 \mathrm{H}), 7.60-7.58(\mathrm{dd}, J=3.6,1.1 \mathrm{~Hz}, 1 \mathrm{H})$, 7.42-7.37 (m, 3H), 7.24-7.22 (m, 1H), 7.11-7.09 (dd, $J=5.0,3.7 \mathrm{~Hz}, 1 \mathrm{H}), 4.44-4.39$ (q, $J=7.1 \mathrm{~Hz}$, 2H), 1.52-1.49 (t, $J=7.1 \mathrm{~Hz}, 3 \mathrm{H}) \mathrm{ppm} ;{ }^{13} \mathrm{C}$ NMR $\left(100 \mathrm{MHz}, \mathrm{CDCl}_{3}\right) \delta 151.2,148.6,131.2,130.4$, 128.4, 127.8, 127.3, 126.5, 125.8, 125.2, 116.9, 70.1, 15.2 ppm; IR (Neat) $v_{\max } 2924,2851,1735$, 1633, 1498, 1448, 1381, 1354, 1201, 1017, 852, $695 \mathrm{~cm}^{-1}$; HRMS (ESI) $\mathrm{m} / z:[\mathrm{M}+\mathrm{H}]^{+}$Calcd for $\mathrm{C}_{15} \mathrm{H}_{14} \mathrm{NO}_{2} \mathrm{~S}$ 272.0740; Found 272.0743.

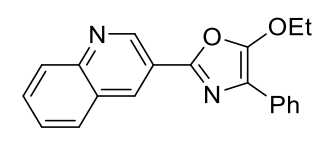

5-Ethoxy-4-phenyl-2-(quinolin-3-yl)oxazole (3j). According to procedure A, 3j was obtained from quinoline-3-carbaldehyde oxime $(34.5 \mathrm{mg}, 0.2 \mathrm{mmol})$ and ethyl 2-diazo-2-phenylacetate (76.5 mg, $0.4 \mathrm{mmol})$ as light yellow oil (24.6 mg, 39\% yield, eluent: petroleum ether/ethyl acetate $=5: 1) .{ }^{1} \mathrm{H}$ NMR $\left(400 \mathrm{MHz}, \mathrm{CDCl}_{3}\right) \delta 9.55-9.54(\mathrm{~d}, J=2.1 \mathrm{~Hz}, 1 \mathrm{H}), 8.70-8.69(\mathrm{~d}, J=1.8 \mathrm{~Hz}, 1 \mathrm{H})$, 8.16-8.14 (d, $J=8.5 \mathrm{~Hz}, 1 \mathrm{H}), 7.97-7.95(\mathrm{dd}, J=3.2,1.3 \mathrm{~Hz}, 2 \mathrm{H}), 7.93-7.91(\mathrm{~d}, J=7.9 \mathrm{~Hz}, 1 \mathrm{H})$, 7.79-7.74 (m, 1H), 7.63-7.59 (td, $J=12.0,1.0 \mathrm{~Hz}, 1 \mathrm{H}), 7.46-7.42(\mathrm{~m}, 2 \mathrm{H}), 7.30-7.28(\mathrm{~m}, 1 \mathrm{H})$, 4.54-4.49 (q, $J=7.1 \mathrm{~Hz}, 2 \mathrm{H}), 1.58-1.55(\mathrm{t}, J=7.1 \mathrm{~Hz}, 3 \mathrm{H}) \mathrm{ppm} ;{ }^{13} \mathrm{C}$ NMR $\left(100 \mathrm{MHz}, \mathrm{CDCl}_{3}\right) \delta$ $154.4,150.0,148.2,147.6,142.8,132.1,130.3,129.5,128.6,128.3,127.5,126.8,126.5,125.2$, 120.9, 117.5, 70.1, 15.2 ppm; IR (Neat) $v_{\max }$ 3061, 2981, 2926, 1738, 1683, 1630, 1602, 1497, 1382, 1354, 1198, 1019, 753, $696 \mathrm{~cm}^{-1}$; HRMS (ESI) $\mathrm{m} / z$ : $[\mathrm{M}+\mathrm{H}]^{+}$Calcd for $\mathrm{C}_{20} \mathrm{H}_{17} \mathrm{~N}_{2} \mathrm{O}_{2}$ : 
317.1285; Found 317.1287.

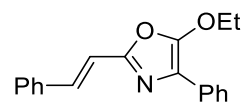

(E)-5-Ethoxy-4-phenyl-2-styryloxazole (3k). According to procedure A, 3k was obtained from (2E)-cinnamaldehyde oxime (29.4 mg, $0.2 \mathrm{mmol})$ and ethyl 2-diazo-2-phenylacetate (76.5 $\mathrm{mg}, 0.4$ mmol) as light yellow oil $(39.6 \mathrm{mg}, 68 \%$ yield, eluent: petroleum ether/ethyl acetate $=20: 1) .{ }^{1} \mathrm{H}$ NMR (400 MHz, $\left.\mathrm{CDCl}_{3}\right) \delta$ 7.88-7.85 (m, 2H), 7.54-7.51 (m, 2H), 7.42-7.37 (m, 5H), 7.34-7.30 (m, 1H), 7.25-7.22 (m, 1H), 6.89-6.85 (d, $J=16.3 \mathrm{~Hz}, 1 \mathrm{H}), 4.47-4.42(\mathrm{q}, J=7.1 \mathrm{~Hz}, 2 \mathrm{H})$, $1.53-1.50(\mathrm{t}, J=7.1 \mathrm{~Hz}, 3 \mathrm{H}) \mathrm{ppm} ;{ }^{13} \mathrm{C} \mathrm{NMR}\left(100 \mathrm{MHz}, \mathrm{CDCl}_{3}\right) \delta 153.6,152.1,135.8,133.9$, $131.4,128.9,128.8,128.5,126.9,126.5,125.0,116.9,114.1,69.7,15.2 \mathrm{ppm}$; IR (Neat) $v_{\max } 3344$, $3179,3059,2926,1735,1685,1628,1495,1448,1282,1353,1382,1199,1019,961,754,594$ $\mathrm{cm}^{-1}$; HRMS (ESI) $m / z$ : [M+H] $]^{+}$Calcd for $\mathrm{C}_{19} \mathrm{H}_{18} \mathrm{NO}_{2} 292.1332$; Found 292.1329 .

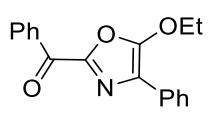

(5-Ethoxy-4-phenyloxazol-2-yl)(phenyl)methanone (3I). According to procedure A, 3I was obtained from 2-oxo-2-phenylacetaldehyde oxime $\left(\begin{array}{llll}30.0 & \mathrm{mg}, & 0.2 \mathrm{mmol}\end{array}\right)$ and ethyl 2-diazo-2-phenylacetate $(76.5 \mathrm{mg}, 0.4 \mathrm{mmol})$ as light yellow oil. $(32.8 \mathrm{mg}, 56 \%$ yield, eluent: petroleum ether/ethyl acetate $=15: 1) .{ }^{1} \mathrm{H}$ NMR $\left(400 \mathrm{MHz}, \mathrm{CDCl}_{3}\right) \delta$ 8.55-8.53 (m, 2H), 7.94-7.92 (m, 2H), 7.64-7.62 (t, $J=7.3 \mathrm{~Hz}, 1 \mathrm{H}), 7.56-7.52(\mathrm{t}, J=7.8 \mathrm{~Hz}, 2 \mathrm{H}), 7.45-7.42(\mathrm{t}, J=7.6 \mathrm{~Hz}, 2 \mathrm{H})$, 7.31-7.27 (t, $J=7.4 \mathrm{~Hz}, 1 \mathrm{H}), 4.68-4.62(\mathrm{q}, J=7.1 \mathrm{~Hz}, 2 \mathrm{H}), 1.59-1.55(\mathrm{t}, J=7.1 \mathrm{~Hz}, 3 \mathrm{H}) \mathrm{ppm} ;{ }^{13} \mathrm{C}$ $\operatorname{NMR}\left(100 \mathrm{MHz}, \mathrm{CDCl}_{3}\right) \delta 177.2,156.3,147.8,135.4,133.4,130.8,130.6,128.6,128.3,127.1$, 125.2, 117.1, 69.5, 15.1 ppm; IR (Neat) $v_{\max } 3063,2981,2933,2159,1974,1751,1496,1444$, 1368, 1262, 1204, 1177, 1061, 1027, 924, 761, $694 \mathrm{~cm}^{-1}$; HRMS (ESI) $\mathrm{m} / z:[\mathrm{M}+\mathrm{H}]^{+}$Calcd for $\mathrm{C}_{18} \mathrm{H}_{16} \mathrm{NO}_{3} 294.1125$; Found 294.1128.

$$
\mathrm{EtO}_{2} \mathrm{C}-\mathrm{C}_{\mathrm{N}}^{\mathrm{O}} \mathrm{I}_{\mathrm{Ph}}^{\mathrm{OEt}}
$$

Ethyl 5-ethoxy-4-phenyloxazole-2-carboxylate $(3 \mathrm{~m})$. According to procedure A, 3m was obtained from ethyl 2-(hydroxyimino)acetate $\quad\left(\begin{array}{lllll}23.4 & \mathrm{mg}, & 0.2 & \mathrm{mmol}\end{array}\right)$ and ethyl 2-diazo-2-phenylacetate $(76.5 \mathrm{mg}, 0.4 \mathrm{mmol})$ as light yellow oil $(31.8 \mathrm{mg}, 61 \%$ yield, eluent: petroleum ether/ethyl acetate $=10: 1) .{ }^{1} \mathrm{H}$ NMR $\left(400 \mathrm{MHz}, \mathrm{CDCl}_{3}\right) \delta$ 7.91-7.89 $(\mathrm{dd}, J=7.1,1.3$ $\mathrm{Hz}, 2 \mathrm{H}), 7.42-7.38(\mathrm{~m}, 2 \mathrm{H}), 7.29-7.24(\mathrm{~m}, 1 \mathrm{H}), 4.57-4.52(\mathrm{q}, J=7.1 \mathrm{~Hz}, 2 \mathrm{H}), 4.50-4.45(\mathrm{q}, J=7.0$ 
$\mathrm{Hz}, 2 \mathrm{H}), 1.54-1.50(\mathrm{t}, J=7.0 \mathrm{~Hz}, 3 \mathrm{H}), 1.46-1.43(\mathrm{t}, J=7.1 \mathrm{~Hz}, 3 \mathrm{H}) \mathrm{ppm} ;{ }^{13} \mathrm{C}$ NMR $(100 \mathrm{MHz}$ $\left.\mathrm{CDCl}_{3}\right) \delta 155.5,155.3,141.9,130.3,128.5,127.1,125.3,117.5,69.7,62.4,15.1,14.3$ ppm; IR (Neat) $v_{\max } 3331,3076,2985,2909,1717,1445,1305,1258,1205,1026,917,855,735 \mathrm{~cm}^{-1}$; HRMS (ESI) $m / z$ : [M+H $]^{+}$Calcd for $\mathrm{C}_{14} \mathrm{H}_{16} \mathrm{NO}_{4}$ 262.1074; Found 262.1079.

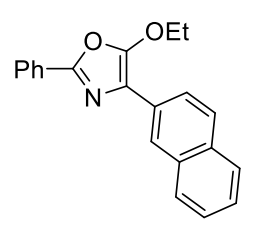

5-Ethoxy-4-(naphthalen-1-yl)-2-phenyloxazole (3n). According to procedure A, 3n was obtained from benzaldehyde oxime $(36.3 \mathrm{mg}, \quad 0.2 \mathrm{mmol})$ and ethyl 2-diazo-2-(naphthalen-1-yl)acetate (96.2 mg, $0.4 \mathrm{mmol}$ ) as light yellow oil (46.1 mg, 73\% yield, eluent: petroleum ether/ethyl acetate $=20: 1) .{ }^{1} \mathrm{H}$ NMR $\left(400 \mathrm{MHz}, \mathrm{CDCl}_{3}\right) \delta 8.40-8.38(\mathrm{~m}, 1 \mathrm{H})$, 8.07-8.05 (m, 2H), 7.89-7.84 (m, 2H), 7.72-7.70 (dd, $J=7.1,1.1 \mathrm{~Hz}, 1 \mathrm{H}), 7.54-7.42(\mathrm{~m}, 6 \mathrm{H})$, 4.27-4.21 (q, $J=7.1 \mathrm{~Hz}, 2 \mathrm{H}), 1.35-1.31(\mathrm{t}, J=7.1 \mathrm{~Hz}, 3 \mathrm{H}) \mathrm{ppm} ;{ }^{13} \mathrm{C}$ NMR $\left(100 \mathrm{MHz}, \mathrm{CDCl}_{3}\right) \delta$ 155.0, 152.2, 134.0, 131.6, 129.7, 128.8, 128.4, 128.4, 128.3, 127.8, 127.5, 126.2, 126.1, 125.8 , 125.6, 125.3, 116.7, 70.1, 15.1 ppm; IR (Neat) $v_{\max } 3046,2981,1736,1628,1484,1388,1325$, 1223, 1173, 1014, 960, 885, 800, 772, 690, $556 \mathrm{~cm}^{-1}$; HRMS (ESI) $m / z:[\mathrm{M}+\mathrm{H}]^{+}$Calcd for $\mathrm{C}_{21} \mathrm{H}_{18} \mathrm{NO}_{2}$ 316.1332; Found 316.1335.

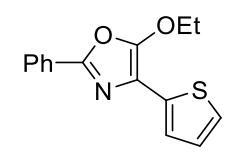

5-Ethoxy-2-phenyl-4-(thiophen-2-yl)oxazole (3o). According to procedure A, 3o was obtained from benzaldehyde oxime (24.2 mg, $0.2 \mathrm{mmol})$ and ethyl 2-diazo-2-(thiophen-2-yl)acetate (78.5 $\mathrm{mg}, 0.4 \mathrm{mmol})$ as light yellow oil $(33.9 \mathrm{mg}, 63 \%$ yield, eluent: petroleum ether/ethyl acetate $=$ 20:1). ${ }^{1} \mathrm{H}$ NMR $\left(400 \mathrm{MHz}, \mathrm{CDCl}_{3}\right) \delta 8.01-7.98(\mathrm{dd}, J=7.9,1.7 \mathrm{~Hz}, 2 \mathrm{H}), 7.47-7.41(\mathrm{~m}, 3 \mathrm{H})$, 7.39-7.38 (dd, $J=3.6,1.1 \mathrm{~Hz}, 1 \mathrm{H}), 7.26-7.24(\mathrm{dd}, J=5.1,1.1 \mathrm{~Hz}, 1 \mathrm{H}), 7.09-7.06$ (dd, $J=5.1,3.6$ $\mathrm{Hz}, 1 \mathrm{H}), 4.46-4.40(\mathrm{q}, J=7.1 \mathrm{~Hz}, 2 \mathrm{H}), 1.53-1.50(\mathrm{t}, J=7.1 \mathrm{~Hz}, 3 \mathrm{H}) \mathrm{ppm} ;{ }^{13} \mathrm{C}$ NMR $(100 \mathrm{MHz}$, $\left.\mathrm{CDCl}_{3}\right) \delta 152.6,152.3,133.9,129.8,128.7,127.4,127.4,125.7,123.6,122.8,114.3,70.2,15.2$ ppm; IR (Neat) $v_{\max } 2982,2927,1739,1645,1529,1448,1319,1236,1204,1015,847,690 \mathrm{~cm}^{-1}$; HRMS (ESI) $m / z$ : $[\mathrm{M}+\mathrm{H}]^{+}$Calcd for $\mathrm{C}_{15} \mathrm{H}_{14} \mathrm{NO}_{2} \mathrm{~S}$ 272.0740; Found 272.0741. 


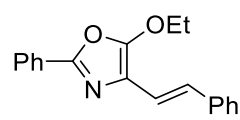

(E)-5-Ethoxy-2-phenyl-4-styryloxazole (3p). According to procedure A, 3p was obtained from benzaldehyde oxime (24.2 $\mathrm{mg}, 0.2 \mathrm{mmol})$ and ethyl $(E)$-2-diazo-4-phenylbut-3-enoate $(86.5 \mathrm{mg}$, $0.4 \mathrm{mmol})$ as light yellow oil $(37.1 \mathrm{mg}, 66 \%$ yield, eluent: petroleum ether/ethyl acetate $=20: 1)$. ${ }^{1} \mathrm{H}$ NMR (400 MHz, $\left.\mathrm{CDCl}_{3}\right) \delta$ 8.00-7.98 (m, 2H), 7.52-7.50 (m, 2H), 7.47-7.41 (m, 3H), 7.36-7.32 $(\mathrm{m}, 2 \mathrm{H}), 7.26-7.20(\mathrm{~m}, 2 \mathrm{H}), 6.93-6.89(\mathrm{~d}, J=16.1 \mathrm{~Hz}, 1 \mathrm{H}), 4.43-4.37(\mathrm{q}, J=7.0 \mathrm{~Hz}, 2 \mathrm{H})$, $1.51-1.48(\mathrm{t}, J=7.0 \mathrm{~Hz}, 3 \mathrm{H}) \mathrm{ppm} ;{ }^{13} \mathrm{C} \mathrm{NMR}\left(100 \mathrm{MHz}, \mathrm{CDCl}_{3}\right) \delta 154.5,152.7,137.6,129.8$, $128.7,128.6,127.5,127.4,127.1,126.2,125.7,117.2,116.0,70.2,15.1 \mathrm{ppm}$; IR (Neat) $v_{\max } 3063$, $2919,2850,1815,1651,1494,1449,1320,1287,1158,1049,998,947,888,778,755,697,662$ $\mathrm{cm}^{-1}$; HRMS (ESI) $m / z:[\mathrm{M}+\mathrm{H}]^{+}$Calcd for $\mathrm{C}_{19} \mathrm{H}_{18} \mathrm{NO}_{2}$ 292.1332; Found 292.1334.<smiles>CCOc1oc(-c2ccccc2)nc1N</smiles>

5-Ethoxy-4-methyl-2-phenyloxazole (3q). According to procedure A, 3q was obtained from benzaldehyde oxime (24.2 $\mathrm{mg}, 0.2 \mathrm{mmol})$ and ethyl 2-diazopropanoate $(51.3 \mathrm{mg}, 0.4 \mathrm{mmol})$ as light yellow oil (28.2 mg, 70\% yield, eluent: petroleum ether/ethyl acetate $=20: 1) .{ }^{1} \mathrm{H}$ NMR (400 $\left.\mathrm{MHz}, \mathrm{CDCl}_{3}\right) \delta$ 7.93-7.90 (m, 2H), 7.44-7.37 (m, 3H), 4.26-4.21 (q, J=7.1 Hz, 2H), 2.12 (s, 3H), $1.43-1.40(\mathrm{t}, J=7.1 \mathrm{~Hz}, 3 \mathrm{H}) \mathrm{ppm} ;{ }^{13} \mathrm{C} \mathrm{NMR}\left(100 \mathrm{MHz}, \mathrm{CDCl}_{3}\right) \delta 154.0,152.3,129.5,128.6$, $127.8,125.3,114.5,70.4,15.0,10.2$ ppm; IR (Neat) $v_{\max } 2983,2935,1727,1618,1577,1497$, 1438, 1138, 1014, 885, 767, $695 \mathrm{~cm}^{-1}$; HRMS (ESI) $m / z$ : $[\mathrm{M}+\mathrm{H}]^{+}$Calcd for $\mathrm{C}_{12} \mathrm{H}_{14} \mathrm{NO}_{2} 204.1019$; Found 204.1020.

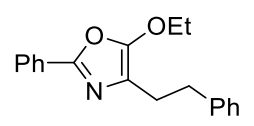

5-Ethoxy-4-phenethyl-2-phenyloxazole (3r). According to procedure A, 3r was obtained from benzaldehyde oxime (24.2 mg, $0.2 \mathrm{mmol})$ and ethyl 2-diazo-4-phenylbutanoate $(87.2 \mathrm{mg}, 0.4$ mmol) as light yellow oil (40.1 mg, 68\% yield, eluent: petroleum ether/ethyl acetate $=20: 1) .{ }^{1} \mathrm{H}$ NMR (400 MHz, $\left.\mathrm{CDCl}_{3}\right) \delta$ 7.95-7.93 (m, 2H), 7.45-7.39 (m, 3H), 7.29-7.25 (m, 2H), 7.22-7.17 (m, 3H), 4.00-3.94 (q, $J=7.1 \mathrm{~Hz}, 2 \mathrm{H}), 3.01-2.97$ (t, $J=7.3 \mathrm{~Hz}, 2 \mathrm{H}), 2.79-2.75(\mathrm{t}, J=7.3 \mathrm{~Hz}, 2 \mathrm{H})$, $1.30-1.27$ (t, $J=7.1 \mathrm{~Hz}, 3 \mathrm{H}) \mathrm{ppm} ;{ }^{13} \mathrm{C} \mathrm{NMR}\left(100 \mathrm{MHz}, \mathrm{CDCl}_{3}\right) \delta 154.3,152.5,141.7,129.5$, $128.6,128.6,128.3,127.9,125.9,125.4,117.9,70.5,34.8,26.9,15.0$ ppm; IR (Neat) $v_{\max } 3061$, 
2926, 2858, 1728, 1654, 1552, 1452, 1377, 1298, 1246, 1070, 1019, 883, 771, $691 \mathrm{~cm}^{-1}$; HRMS (ESI) $m / z:[\mathrm{M}+\mathrm{H}]^{+}$Calcd for $\mathrm{C}_{19} \mathrm{H}_{20} \mathrm{NO}_{2}$ 294.1489; Found 294.1490.<smiles>COC(=O)CCc1nc(-c2ccccc2)oc1OC</smiles>

Methyl 3-(5-methoxy-2-phenyloxazol-4-yl)propanoate (3s). According to procedure A, 3s was obtained from benzaldehyde oxime ( $24.2 \mathrm{mg}, 0.2 \mathrm{mmol})$ and dimethyl 2-diazopentanedioate (75.1 $\mathrm{mg}, 0.4 \mathrm{mmol})$ as light yellow oil $(27.6 \mathrm{mg}, 53 \%$ yield, eluent: petroleum ether/ethyl acetate $=$ 15:1). ${ }^{1} \mathrm{H}$ NMR (400 MHz, $\mathrm{CDCl}_{3}$ ) $\delta$ 7.91-7.89 (m, 2H), 7.42-7.39 (m, 3H), 4.00 (s, 3H), 3.69 (s, $3 \mathrm{H}), 2.83-2.79(\mathrm{~m}, 2 \mathrm{H}), 2.73-2.69(\mathrm{~m}, 2 \mathrm{H}) \mathrm{ppm} ;{ }^{13} \mathrm{C} \mathrm{NMR}\left(100 \mathrm{MHz}, \mathrm{CDCl}_{3}\right) \delta$ 173.3, 154.9, 152.2, 130.0, 129.6, 128.7, 125.4, 115.7, 61.2, 51.6, 32.7, $20.3 \mathrm{ppm}$; IR (Neat) $v_{\max } 3366,3008$, 2950, 2853, 2158, 1969, 1735, 1660, 1437, 1327, 1156, 983, $692 \mathrm{~cm}^{-1}$; HRMS (ESI) $\mathrm{m} / z:[\mathrm{M}+\mathrm{H}]^{+}$ Calcd for $\mathrm{C}_{14} \mathrm{H}_{16} \mathrm{NO}_{3} 262.1074$; Found 262.1076.

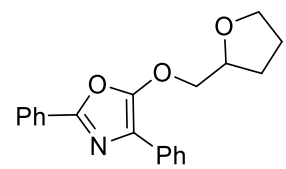

2,4-Diphenyl-5-((tetrahydrofuran-2-yl)methoxy)oxazole (3t). According to procedure A, 3t was obtained from benzaldehyde oxime $(24.2 \mathrm{mg}, 0.2 \mathrm{mmol})$ and (tetrahydrofuran-2-yl)methyl 2-diazo-2-phenylacetate $(98.6 \mathrm{mg}, 0.4 \mathrm{mmol})$ as light yellow oil $(38.2 \mathrm{mg}, 60 \%$ yield, eluent: petroleum ether/ethyl acetate $=20: 1) .{ }^{1} \mathrm{H}$ NMR $\left(400 \mathrm{MHz}, \mathrm{CDCl}_{3}\right) \delta$ 8.02-7.99 $(\mathrm{m}, 2 \mathrm{H})$, 7.94-7.91 (m, 2H), 7.47-7.39 (m, 5H), 7.26-7.22 (m, 1H), 4.37-4.33 (m, 3H), 3.98-3.92 (m, 1H), 3.89-3.83 (m, 1H), 2.14-2.07 (m, 1H), 2.02-1.92 (m, 2H), 1.88-1.80 (m, 1H) ppm; ${ }^{13} \mathrm{C}$ NMR (100 MHz, $\left.\mathrm{CDCl}_{3}\right) \delta 153.9,152.0,131.4,129.7,128.7,128.5,127.6,126.5,125.6,125.3,116.8,76.8,75.5$, 68.7, 28.0, 25.8 ppm; IR (Neat) $v_{\max } 3060,2952,2878,1739,1681,1632,1600,1497,1448,1360$, 1327, 1202, 1071, 1017, 770, $691 \mathrm{~cm}^{-1}$; HRMS (ESI) $m / z:[\mathrm{M}+\mathrm{H}]^{+}$Calcd for $\mathrm{C}_{20} \mathrm{H}_{20} \mathrm{NO}_{3} 322.1438$; Found 322.1440 .

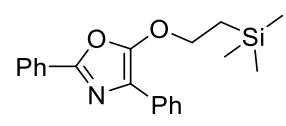

2,4-Diphenyl-5-(2-(trimethylsilyl)ethoxy)oxazole $(3 u)$. According to procedure A, 3u was obtained from benzaldehyde oxime $(24.2 \mathrm{mg}, 0.2 \mathrm{mmol})$ and 2-(trimethylsilyl)ethyl 2-diazo-2-phenylacetate $(105.3 \mathrm{mg}, 0.4 \mathrm{mmol})$ as light yellow oil $(43.1 \mathrm{mg}, 64 \%$ yield, eluent: 
petroleum ether/ethyl acetate $=20: 1) .{ }^{1} \mathrm{H}$ NMR $\left(400 \mathrm{MHz}, \mathrm{CDCl}_{3}\right) \delta 8.22-8.20(\mathrm{~m}, 2 \mathrm{H}), 8.14-8.12$ (m, 2H), 7.67-7.58 (m, 5H), 7.46-7.42 (m, 1H), 4.68-4.63 (m, 2H), 1.50-1.45 (m, 2H), 0.29 (s, 9H) ppm; ${ }^{13} \mathrm{C} \mathrm{NMR}\left(100 \mathrm{MHz}, \mathrm{CDCl}_{3}\right) \delta 153.9,152.0,131.6,129.6,128.7,128.4,127.7,126.4,125.5$, 125.1, 117.1, 72.3, 18.7, -1.51 ppm; IR (Neat) $v_{\max } 3388,3192,2953,2897,2107,1734,1688$, 1630, 1448, 1249, 1172, 918, 856, 835, 768, $689 \mathrm{~cm}^{-1}$; HRMS (ESI) $\mathrm{m} / z:[\mathrm{M}+\mathrm{H}]^{+}$Calcd for $\mathrm{C}_{20} \mathrm{H}_{24} \mathrm{NO}_{2} \mathrm{Si} 338.1571$; Found 338.1574.

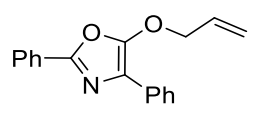

5-(Allyloxy)-2,4-diphenyloxazole (3v). According to procedure A, 3v was obtained from benzaldehyde oxime (24.2 $\mathrm{mg}, 0.2 \mathrm{mmol})$ and allyl 2-diazo-2-phenylacetate $(81.4 \mathrm{mg}, 0.4 \mathrm{mmol})$ as light yellow oil (38.7 mg, 70\% yield, eluent: petroleum ether/ethyl acetate $=20: 1)$. ${ }^{1} \mathrm{H}$ NMR (400 MHz, DMSO- $\left.d_{6}\right) \delta$ 8.06-8.03 (m, 2H), 7.74-7.70 (m, 1H), 7.64-7.60 (m, 4H), 7.52-7.42 (m, 2H), 7.40-7.36 (m, 1H), 5.67-5.56 (m, 1H), 5.21-5.11 (m, 2H), 3.03-2.95 (m, 2H) ppm; ${ }^{13} \mathrm{C}$ NMR $\left(100 \mathrm{MHz}, \mathrm{DMSO}-d_{6}\right) \delta 178.2,160.1,137.7,133.8,131.4,129.7,129.3,128.9,128.3,126.1$, 125.6, 121.4, 74.6, 43.6 ppm; IR (Neat) $v_{\max }$ 3359, 3061, 2982, 2931, 1726, 1659, 1604, 1575, 1448, 1373, 1258, 1079, 1024, 745, 694, $570 \mathrm{~cm}^{-1}$; HRMS (ESI) $m / z:[\mathrm{M}+\mathrm{H}]^{+}$Calcd for $\mathrm{C}_{18} \mathrm{H}_{16} \mathrm{NO}_{2} 278.1176$; Found 278.1175.

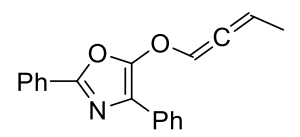

5-(Buta-1,2-dien-1-yloxy)-2,4-diphenyloxazole (3w). According to procedure A, 3w was obtained from benzaldehyde oxime $(24.2 \mathrm{mg}, \quad 0.2 \mathrm{mmol})$ and but-2-yn-1-yl 2-diazo-2-phenylacetate $(85.6 \mathrm{mg}, 0.4 \mathrm{mmol})$ as light yellow oil $(36.4 \mathrm{mg}, 63 \%$ yield, eluent: petroleum ether/ethyl acetate $=20: 1) .{ }^{1} \mathrm{H}$ NMR $\left(400 \mathrm{MHz}, \mathrm{DMSO}-d_{6}\right) \delta 8.08-8.06(\mathrm{~m}, 2 \mathrm{H})$, 7.75-7.71 (m, 1H), 7.65-7.61 (m, 2H), 7.56-7.54 (m, 2H), 7.48-7.39 (m, 3H), 5.11-5.01 (m, 2H), $1.66-1.65(\mathrm{t}, J=3.1 \mathrm{~Hz}, 3 \mathrm{H}) \mathrm{ppm} ;{ }^{13} \mathrm{C}$ NMR (100 MHz, DMSO- $\left.d_{6}\right) \delta 205.7,175.2,160.9,136.3$, 134.0, 129.8, 129.3, 129.2, 128.3, 126.8, 125.5, 99.3, 79.9, 76.4, 15.1 ppm; IR (Neat) $v_{\max } 3062$, 2925, 2856, 1958, 1826, 1648, 1600, 1492, 1449, 1320, 1291, 1134, 1062, 988, 932, 884, $689 \mathrm{~cm}^{-1}$; HRMS (ESI) $m / z$ : $[\mathrm{M}+\mathrm{H}]^{+}$Calcd for $\mathrm{C}_{19} \mathrm{H}_{16} \mathrm{NO}_{2}$ 290.1176; Found 290.1177 .

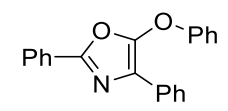

5-Phenoxy-2,4-diphenyloxazole (3x). According to procedure A, 3x was obtained from 
benzaldehyde oxime ( $24.2 \mathrm{mg}, 0.2 \mathrm{mmol})$ and phenyl 2-diazo-2-phenylacetate (95.2 $\mathrm{mg}, 0.4 \mathrm{mmol})$ as light yellow oil (32.5 mg, 52\% yield, eluent: petroleum ether/ethyl acetate $=20: 1) .{ }^{1} \mathrm{H}$ NMR $\left(400 \mathrm{MHz}, \mathrm{CDCl}_{3}\right) \delta$ 8.05-8.03 (m, 2H), 7.92-7.90 (m, 2H), 7.46-7.43 (m, 3H), 7.41-7.34 (m, 4H), 7.29-7.24 (m, 1H), 7.17-7.11 (m, 3H) ppm; ${ }^{13} \mathrm{C}$ NMR (100 MHz, $\left.\mathrm{CDCl}_{3}\right) \delta 156.3,154.3,149.3$, $130.3,130.3,130.0,128.7,128.6,127.4,127.3,126.0,125.6,124.2,122.2,116.1 \mathrm{ppm}$; IR (Neat) $v_{\max } 3061,2926,1965,1641,1589,1488,1448,1372,1327,1208,1023,773,746,711,688 \mathrm{~cm}^{-1}$; HRMS (ESI) $m / z$ : $[\mathrm{M}+\mathrm{H}]^{+}$Calcd for $\mathrm{C}_{21} \mathrm{H}_{16} \mathrm{NO}_{2}$ 314.1176; Found 314.1177.

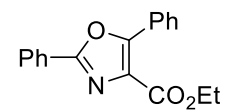

Ethyl 2,5-diphenyloxazole-4-carboxylate (3y). According to procedure A, 3y was obtained from benzaldehyde oxime (24.2 mg, $0.2 \mathrm{mmol})$ and ethyl 2-diazo-3-oxo-3-phenylpropanoate ( $87.3 \mathrm{mg}$, $0.4 \mathrm{mmol})$ as light yellow oil $(38.6 \mathrm{mg}, 66 \%$ yield, eluent: petroleum ether/ethyl acetate $=20: 1)$. ${ }^{1} \mathrm{H}$ NMR (400 MHz, $\left.\mathrm{CDCl}_{3}\right) \delta 8.18-8.16$ (m, 2H), 8.13-8.10 (m, 2H), 7.53-7.47 (m, 6H), 4.49-4.44 $(\mathrm{q}, J=7.2 \mathrm{~Hz}, 2 \mathrm{H}), 1.45-1.41(\mathrm{t}, J=7.2 \mathrm{~Hz}, 3 \mathrm{H}) \mathrm{ppm} ;{ }^{13} \mathrm{C}$ NMR $\left(100 \mathrm{MHz}, \mathrm{CDCl}_{3}\right) \delta 162.3$, $159.8,155.1,131.1,130.3,128.8,128.6,128.4,128.3,127.2,126.9,126.4,61.5,14.3$ ppm; IR (Neat) $v_{\max } 3059,2982,2930,1719,1561,1491,1448,1372,1322,1214,1096,1023,776,710$, $689 \mathrm{~cm}^{-1}$; HRMS (ESI) $\mathrm{m} / z$ : $[\mathrm{M}+\mathrm{H}]^{+}$Calcd for $\mathrm{C}_{18} \mathrm{H}_{16} \mathrm{NO}_{3}$ 294.1125; Found 294.1126 .

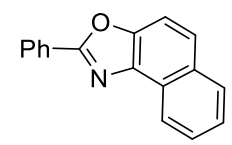

2-Phenylnaphtho[1,2-d]oxazole (3z). According to procedure A, 3z was obtained from benzaldehyde oxime (24.2 mg, $0.2 \mathrm{mmol})$ and 1-diazonaphthalen-2(1H)-one (68.1 mg, $0.4 \mathrm{mmol})$ as light yellow oil $(29.4 \mathrm{mg}, 60 \%$ yield, eluent: petroleum ether/ethyl acetate $=20: 1) .{ }^{1} \mathrm{H}$ NMR $\left(400 \mathrm{MHz}, \mathrm{CDCl}_{3}\right) \delta 8.61-8.58(\mathrm{~m}, 1 \mathrm{H}), 8.35-8.33(\mathrm{~m}, 2 \mathrm{H}), 7.99-7.97(\mathrm{~d}, J=8.2 \mathrm{~Hz}, 1 \mathrm{H})$, 7.83-7.73 (m, 2H), 7.70-7.66 (m, 1H), 7.58-7.53 (m, 4H) ppm; ${ }^{13} \mathrm{C}$ NMR $\left(100 \mathrm{MHz}, \mathrm{CDCl}_{3}\right) \delta$ $162.3,148.1,137.6,131.2,131.1,128.9,128.6,127.5,127.3,127.0,126.6,126.0,125.4,122.3$, 110.8 ppm; IR (Neat) $v_{\max } 3063,2924,2852,2161,2016,1640,1551,1486,1450,1373,1239$, 1089, 1007, 801, 775, 708, $687 \mathrm{~cm}^{-1}$; HRMS (ESI) $\mathrm{m} / z$ : $[\mathrm{M}+\mathrm{H}]^{+}$Calcd for $\mathrm{C}_{17} \mathrm{H}_{12} \mathrm{NO} 246.0913$; Found 246.0915. 


\section{General procedure for the synthesis of oxime ethers (Procedure B)}

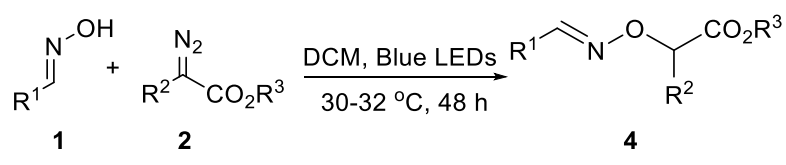

A $10 \mathrm{~mL}$ glass tube equipped with magnetic stir bar was charged with oxime $1(0.2 \mathrm{mmol}, 1.0$ equiv.), $\alpha$-diazoester 2 ( $0.24 \mathrm{mmol}, 1.2$ equiv) under argon atmosphere. The tube was evacuated and backfilled with argon atmosphere for 3 times. Degassed DCM $(2.5 \mathrm{~mL})$ was added by syringe under argon atmosphere. The mixture was then irradiated by $12 \mathrm{~W}$ blue LEDs (purchased from Lingke Lighting Enterprise Store) and stirred at room temperature for 48 hours. Upon completion, the reaction mixture was concentrated under vacuum and the residue was purified by column chromatography (petroleum ether and ethyl acetate as eluents, the ratio is from 15:1 to 4:1) to give the desired product to afford the 4 .
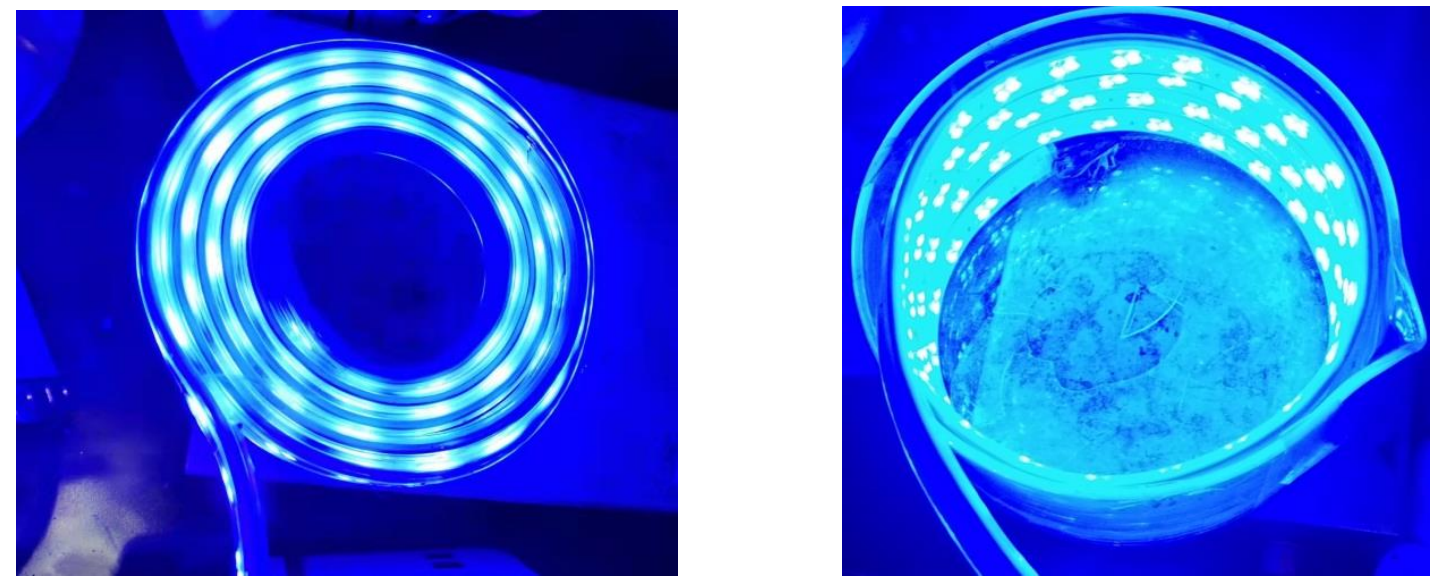

Figure S1. Photoreaction setup in our lab with $12 \mathrm{~W}$ blue LED light strips (spectral range of $435-445 \mathrm{~nm}$ ) taped on the inner wall of the glass cylinder.

\section{Characterization data of oxime ethers $4 a-4 z$}<smiles>CCOC(ON=Cc1ccccc1)c1ccccc1</smiles>

Ethyl (E)-2-((benzylideneamino)oxy)-2-phenylacetate (4a) $)^{5}$. According to procedure B, 4a was obtained from benzaldehyde oxime $(24.2 \mathrm{mg}, 0.2 \mathrm{mmol})$ and ethyl 2-diazo-2-phenylacetate (45.6 $\mathrm{mg}, 0.24 \mathrm{mmol})$ as light yellow oil $(48.2 \mathrm{mg}, 85 \%$ yield, eluent: petroleum ether/ethyl acetate $=$ 15:1). ${ }^{1} \mathrm{H}$ NMR $\left(400 \mathrm{MHz}, \mathrm{CDCl}_{3}\right) \delta 8.27(\mathrm{~s}, 1 \mathrm{H}), 7.59-7.57(\mathrm{~m}, 2 \mathrm{H}), 7.54-7.52(\mathrm{~m}, 2 \mathrm{H})$, 7.42-7.34 (m, 6H), $5.71(\mathrm{~s}, 1 \mathrm{H}), 4.28-4.18(\mathrm{~m}, 2 \mathrm{H}), 1.26-1.22(\mathrm{t}, J=7.1 \mathrm{~Hz}, 3 \mathrm{H}) \mathrm{ppm} ;{ }^{13} \mathrm{C}$ NMR $\left(100 \mathrm{MHz}, \mathrm{CDCl}_{3}\right) \delta 170.5,150.6,134.8,131.7,131.2,130.2,129.0,128.6,127.7,127.3,83.8$, 
61.2, $14.1 \mathrm{ppm}$; IR (Neat) $v_{\max } 2981,2930,1750,1606,1484,1450,1205,1064,903,857,752$, $692 \mathrm{~cm}^{-1}$; HRMS (ESI) m/z: [M+H] $]^{+}$Calcd for $\mathrm{C}_{17} \mathrm{H}_{18} \mathrm{NO}_{3}$ 284.1281; Found 284.1282.<smiles>CCOC(O/N=C/c1ccc2ccccc2c1)c1ccccc1</smiles>

Ethyl (E)-2-(((naphthalen-2-ylmethylene)amino)oxy)-2-phenylacetate (4b). According to procedure $\mathrm{B}, \mathbf{4} \mathbf{b}$ was obtained from 2-naphthaldehyde oxime $(34.3 \mathrm{mg}, 0.2 \mathrm{mmol})$ and ethyl 2-diazo-2-phenylacetate $(45.6 \mathrm{mg}, 0.24 \mathrm{mmol})$ as light yellow oil $(55.2 \mathrm{mg}, 83 \%$ yield, eluent: petroleum ether/ethyl acetate $=15: 1) .{ }^{1} \mathrm{H}$ NMR $\left(400 \mathrm{MHz}, \mathrm{CDCl}_{3}\right) \delta 8.41(\mathrm{~s}, 1 \mathrm{H}), 7.87-7.79(\mathrm{~m}$, 5H), 7.57-7.54 (m, 2H), 7.52-7.47 (m, 2H), 7.43-7.38 (m, 3H), $5.76(\mathrm{~s}, 1 \mathrm{H}), 4.32-4.19(\mathrm{~m}, 2 \mathrm{H})$, 1.27-1.24 (t, $J=7.1 \mathrm{~Hz}, 3 \mathrm{H}) \mathrm{ppm} ;{ }^{13} \mathrm{C} \mathrm{NMR}\left(100 \mathrm{MHz}, \mathrm{CDCl}_{3}\right) \delta 170.6,150.7,134.8,134.2$, $133.1,129.3,129.0,128.9,128.7,128.5,128.4,127.8,127.7,127.0,126.6,123.0,83.9,61.3,14.1$ ppm; IR (Neat) $v_{\max } 3061,2981,2158,2019,1750,1454,1267,1178,1066,945,747,697,475$ $\mathrm{cm}^{-1}$; HRMS (ESI) $m / z$ : $[\mathrm{M}+\mathrm{H}]^{+}$Calcd for $\mathrm{C}_{21} \mathrm{H}_{20} \mathrm{NO}_{3} 334.1438$; Found 334.1440 .<smiles>CCOC(=O)c1ccccc1O/N=C/c1c2ccccc2cc2ccccc12</smiles>

Ethyl (E)-2-(((anthracen-9-ylmethylene)amino)oxy)-2-phenylacetate (4c). According to procedure, $4 \mathbf{c}$ was obtained from anthracene-9-carbaldehyde oxime (44.4 mg, $0.2 \mathrm{mmol}$ ) and ethyl 2-diazo-2-phenylacetate $(45.6 \mathrm{mg}, 0.24 \mathrm{mmol})$ as light yellow oil $(53.6 \mathrm{mg}, 70 \%$ yield, eluent: petroleum ether/ethyl acetate $=15: 1) .{ }^{1} \mathrm{H}$ NMR $\left(400 \mathrm{MHz}\right.$, DMSO- $\left.d_{6}\right) \delta 8.70(\mathrm{~d}, J=2.0 \mathrm{~Hz}, 2 \mathrm{H})$, 8.14-8.12 (m, 4H), 7.63-7.55 (m, 4H), 7.22-7.12 (m, 5H), $5.77(\mathrm{~s}, 1 \mathrm{H}), 4.24-4.14(\mathrm{~m}, 2 \mathrm{H})$, $1.23-1.19(\mathrm{t}, J=7.1 \mathrm{~Hz}, 3 \mathrm{H}) \mathrm{ppm} ;{ }^{13} \mathrm{C}$ NMR $\left(100 \mathrm{MHz}, \mathrm{DMSO}-d_{6}\right) \delta 170.5,148.0,134.5,131.1$, 130.0, 129.2, 129.1, 128.8, 128.6, 128.0, 127.5, 126.9, 126.1, 125.4, 83.5, 61.4, 14.5 ppm; IR (Neat) $v_{\max } 3360,3053,2921,1750,1454,1263,1206,1178,1095,1046,912,732,696 \mathrm{~cm}^{-1}$; HRMS (ESI) $m / z$ : [M+H $]^{+}$Calcd for $\mathrm{C}_{25} \mathrm{H}_{22} \mathrm{NO}_{3}$ 384.1594; Found 384.1595.<smiles>CCOC(O/N=C/c1cnc2ccccc2c1)c1ccccc1</smiles>

Ethyl (E)-2-phenyl-2-(((quinolin-3-ylmethylene)amino)oxy)acetate (4d). According to procedure B, 4d was obtained from quinoline-3-carbaldehyde oxime (34.5 mg, $0.2 \mathrm{mmol})$ and ethyl 2-diazo-2-phenylacetate $(45.6 \mathrm{mg}, 0.24 \mathrm{mmol})$ as light yellow oil $(38.7 \mathrm{mg}, 58 \%$ yield, 
eluent: petroleum ether/ethyl acetate $=15: 1) .{ }^{1} \mathrm{H}$ NMR $\left(400 \mathrm{MHz}, \mathrm{CDCl}_{3}\right) \delta 9.20-9.19(\mathrm{~d}, J=2.0$ $\mathrm{Hz}, 1 \mathrm{H}), 8.43(\mathrm{~s}, 1 \mathrm{H}), 8.23(\mathrm{~d}, J=1.9 \mathrm{~Hz}, 1 \mathrm{H}), 8.12-8.10(\mathrm{~d}, J=8.2 \mathrm{~Hz}, 1 \mathrm{H}), 7.85$ (dd, $J=8.2,0.9$ Hz, 1H), 7.77-7.73 (m, 1H), 7.60-7.54 (m, 3H), 7.45-7.39 (m, 3H), $5.79(\mathrm{~s}, 1 \mathrm{H}), 4.33-4.19(\mathrm{~m}, 2 \mathrm{H})$, $1.28-1.25(\mathrm{t}, J=7.2 \mathrm{~Hz}, 3 \mathrm{H}) \mathrm{ppm} ;{ }^{13} \mathrm{C} \mathrm{NMR}\left(100 \mathrm{MHz}, \mathrm{CDCl}_{3}\right) \delta 170.3,148.6,148.5,148.1$, $134.9,134.5,130.5,129.5,129.2,128.7,128.2,127.8,127.5,127.3,124.9,84.1,61.4,14.1 \mathrm{ppm}$; IR (Neat) $v_{\max } 3064,2981,2932,1748,1492,1264,1206,1178,1065,952,786,752,697 \mathrm{~cm}^{-1}$; HRMS (ESI) $m / z$ : [M+H] $]^{+}$Calcd for $\mathrm{C}_{20} \mathrm{H}_{19} \mathrm{~N}_{2} \mathrm{O}_{3}$ 335.1390; Found 335.1393<smiles>CCOC(ON=Cc1ccco1)C(OCC)c1ccccc1</smiles>

Ethyl (E)-2-(((furan-2-ylmethylene)amino)oxy)-2-phenylacetate (4e). According to procedure B, 4e was obtained from furan-2-carbaldehyde oxime $(22.3 \mathrm{mg}, 0.2 \mathrm{mmol})$ and ethyl 2-diazo-2-phenylacetate $(45.6 \mathrm{mg}, 0.24 \mathrm{mmol})$ as light yellow oil $(42.0 \mathrm{mg}, 77 \%$ yield, eluent: petroleum ether/ethyl acetate $=15: 1) .{ }^{1} \mathrm{H}$ NMR $\left(400 \mathrm{MHz}, \mathrm{CDCl}_{3}\right) \delta 8.14(\mathrm{~s}, 1 \mathrm{H}), 7.51-7.49(\mathrm{~m}$, 3H), 7.39-7.37 (m, 3H), 6.66-6.65 (d, $J=3.2 \mathrm{~Hz}, 1 \mathrm{H}), 6.47-6.45$ (m, 1H), $5.71(\mathrm{~s}, 1 \mathrm{H}), 4.27-4.17$ (m, 2H), 1.25-1.21 (t, $J=7.2 \mathrm{~Hz}, 3 \mathrm{H}) \mathrm{ppm} ;{ }^{13} \mathrm{C} \mathrm{NMR}\left(100 \mathrm{MHz}, \mathrm{CDCl}_{3}\right) \delta 170.3,146.6,144.6$, 140.8, 134.7, 129.1, 128.7, 127.8, 113.8, 111.7, 83.9, 61.3, 14.1 ppm; IR (Neat) $v_{\max } 3126,2981$, 2922, 1749, 1482, 1266, 1206, 1178, 1065, 929, 745, $696 \mathrm{~cm}^{-1}$; HRMS (ESI) $\mathrm{m} / z$ : $[\mathrm{M}+\mathrm{H}]^{+} \mathrm{Calcd}$ for $\mathrm{C}_{15} \mathrm{H}_{16} \mathrm{NO}_{4} 274.1074$; Found 274.1076.

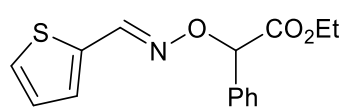

Ethyl (E)-2-phenyl-2-(((thiophen-2-ylmethylene)amino)oxy)acetate (4f). According to procedure B, $4 \mathbf{f}$ was obtained from thiophene-2-carbaldehyde oxime $(25.4 \mathrm{mg}, 0.2 \mathrm{mmol})$ and ethyl 2-diazo-2-phenylacetate $(45.6 \mathrm{mg}, 0.24 \mathrm{mmol})$ as light yellow oil $(42.9 \mathrm{mg}, 74 \%$ yield, eluent: petroleum ether/ethyl acetate $=15: 1) .{ }^{1} \mathrm{H} \mathrm{NMR}\left(400 \mathrm{MHz}, \mathrm{CDCl}_{3}\right) \delta 7.73(\mathrm{~d}, J=0.4 \mathrm{~Hz}$, 1H), 7.62-7.60 (m, 2H), 7.57-7.56 (td, $J=5.1,2.0 \mathrm{~Hz}, 1 \mathrm{H}), 7.44-7.38(\mathrm{~m}, 4 \mathrm{H}), 7.10-7.07(\mathrm{dd}, J=$ 8.8, $5.1 \mathrm{~Hz}, 1 \mathrm{H}), 5.76(\mathrm{~s}, 1 \mathrm{H}), 4.29-4.15(\mathrm{~m}, 2 \mathrm{H}), 1.25-1.21(\mathrm{t}, J=7.1 \mathrm{~Hz}, 3 \mathrm{H}) \mathrm{ppm} ;{ }^{13} \mathrm{C} \mathrm{NMR}$ $\left(100 \mathrm{MHz}, \mathrm{CDCl}_{3}\right) \delta 170.1,141.9,135.0,132.5,132.2,131.0,129.0,128.6,127.7,126.3,84.6$, 61.4, 14.1 ppm; IR (Neat) $v_{\max } 2981,2934,2159,1750,1613,1265,1208,1178,1093,1051,920$, 725, $696 \mathrm{~cm}^{-1}$; HRMS (ESI) m/z: [M+H] $]^{+}$Calcd for $\mathrm{C}_{15} \mathrm{H}_{16} \mathrm{NO}_{3} \mathrm{~S} 290.0845$; Found 290.0847. 


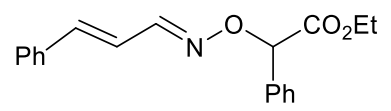

Ethyl 2-phenyl-2-((((1E,2E)-3-phenylallylidene)amino)oxy)acetate (4g). According to procedure B, $4 \mathbf{g}$ was obtained from (2E)-cinnamaldehyde oxime (29.5 $\mathrm{mg}, 0.2 \mathrm{mmol})$ and ethyl 2-diazo-2-phenylacetate $(45.6 \mathrm{mg}, 0.24 \mathrm{mmol})$ as light yellow oil $(40.1 \mathrm{mg}, 65 \%$ yield, eluent: petroleum ether/ethyl acetate $=15: 1) .{ }^{1} \mathrm{H}$ NMR $\left(400 \mathrm{MHz}, \mathrm{CDCl}_{3}\right) \delta$ 7.55-7.53 $(\mathrm{m}, 2 \mathrm{H}), 7.51-7.49$ (m, 2H), 7.45-7.32 (m, 6H), 7.29-7.27 (d, $J=8.0 \mathrm{~Hz}, 1 \mathrm{H}), 6.90-6.86(\mathrm{~d}, J=16.0 \mathrm{~Hz}, 1 \mathrm{H}), 5.65(\mathrm{~s}$, 1H), 4.27-4.16 (m, 2H), 1.26-1.23 (t, $J=7.1 \mathrm{~Hz}, 3 \mathrm{H}) \mathrm{ppm} ;{ }^{13} \mathrm{C}$ NMR (100 MHz, $\left.\mathrm{CDCl}_{3}\right) \delta 170.5$, $149.7,140.9,135.6,135.0,129.5,129.0,128.8,128.6,127.8,127.6,116.4,83.7,61.2,14.1 \mathrm{ppm}$; IR (Neat) $v_{\max }$ 3062, 2981, 2930, 1750, 1626, 1494, 1449, 1205, 1064, 903, 857, 752, $692 \mathrm{~cm}^{-1}$; HRMS (ESI) $m / z$ : [M+H] $]^{+}$Calcd for $\mathrm{C}_{19} \mathrm{H}_{20} \mathrm{NO}_{3}$ 310.1438; Found 310.1441.<smiles>CCOC(ON=CC(C)=O)c1ccccc1</smiles>

Ethyl (E)-2-(((2-oxopropylidene)amino)oxy)-2-phenylacetate (4h). According to procedure B, 4h was obtained from 2-oxopropanal oxime (17.4 $\mathrm{mg}, \quad 0.2 \mathrm{mmol})$ and ethyl 2-diazo-2-phenylacetate $(45.6 \mathrm{mg}, 0.24 \mathrm{mmol})$ as light yellow oil $(37.5 \mathrm{mg}, 75 \%$ yield, eluent: petroleum ether/ethyl acetate $=15: 1) .{ }^{1} \mathrm{H}$ NMR $\left(400 \mathrm{MHz}, \mathrm{CDCl}_{3}\right) \delta 7.87(\mathrm{~s}, 1 \mathrm{H}), 7.50-7.47(\mathrm{~m}$, 2H), 7.39-7.38 (m, 3H), $5.65(\mathrm{~s}, 1 \mathrm{H}), 4.28-4.18(\mathrm{~m}, 2 \mathrm{H}), 2.04(\mathrm{~s}, 3 \mathrm{H}), 1.26-1.22(\mathrm{t}, J=7.1 \mathrm{~Hz}, 3 \mathrm{H})$ ppm; ${ }^{13} \mathrm{C}$ NMR (100 MHz, $\left.\mathrm{CDCl}_{3}\right) \delta 170.3,153.8,149.2,134.4,129.1,128.7,127.7,84.1,61.4$, 14.1, 9.2 ppm; IR (Neat) $v_{\max } 3064,2981,2934,2160,1751,1455,1369,1264,1205,1178,1091$, 1066, 933, $695 \mathrm{~cm}^{-1}$; HRMS (ESI) $\mathrm{m} / z$ : $[\mathrm{M}+\mathrm{Na}]^{+}$Calcd for $\mathrm{C}_{13} \mathrm{H}_{15} \mathrm{NNaO}_{4}$ 272.0893; Found 272.0889 .<smiles>CCOC(=O)C=NOC(C(=O)OCC)c1ccccc1</smiles>

Ethyl (E)-2-(((2-ethoxy-2-oxoethylidene)amino)oxy)-2-phenylacetate (4i). According to procedure B, 4i was obtained from ethyl 2-(hydroxyimino)acetate (23.6 mg, $0.2 \mathrm{mmol}$ ) and ethyl 2-diazo-2-phenylacetate $(45.6 \mathrm{mg}, 0.24 \mathrm{mmol})$ as light yellow oil $(39.9 \mathrm{mg}, 70 \%$ yield, eluent: petroleum ether/ethyl acetate $=15: 1) .{ }^{1} \mathrm{H}$ NMR $\left(400 \mathrm{MHz}, \mathrm{CDCl}_{3}\right) \delta 7.67(\mathrm{~s}, 1 \mathrm{H}), 7.47-7.45(\mathrm{~m}$, 2H), 7.41-7.39 (m, 3H), 5.79 (s, 1H), 4.34-4.29 (q, $J=7.1 \mathrm{~Hz}, 2 \mathrm{H}), 4.27-4.15(\mathrm{~m}, 2 \mathrm{H}), 1.35-1.32$ (t, $J=7.2 \mathrm{~Hz}, 3 \mathrm{H}), 1.25-1.21(\mathrm{t}, J=7.1 \mathrm{~Hz}, 3 \mathrm{H}) \mathrm{ppm} ;{ }^{13} \mathrm{C} \mathrm{NMR}\left(100 \mathrm{MHz}, \mathrm{CDCl}_{3}\right) \delta 169.5,161.5$, $142.7,133.8,129.4,128.8,127.8,84.7,61.8,61.6,14.1,14.0 \mathrm{ppm}$; IR (Neat) $v_{\max } 2982,2938$, 
1743, 1606, 1455, 1370, 1323, 1263, 1200, 1063, 1028, 961, 854, 727, $697 \mathrm{~cm}^{-1}$; HRMS (ESI) $m / z$ : $[\mathrm{M}+\mathrm{H}]^{+}$Calcd for $\mathrm{C}_{14} \mathrm{H}_{18} \mathrm{NO}_{5} 280.1179$; Found 280.1180 .

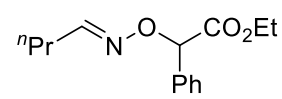

Ethyl (E)-2-((butylideneamino)oxy)-2-phenylacetate $(\mathbf{4 j})$. According to procedure B, $\mathbf{4 j}$ was obtained from butyraldehyde oxime $(20.2 \mathrm{mg}, 0.2 \mathrm{mmol})$ and ethyl 2-diazo-2-phenylacetate (45.6 $\mathrm{mg}, 0.24 \mathrm{mmol})$ as light yellow oil $(30.5 \mathrm{mg}, 61 \%$ yield, eluent: petroleum ether/ethyl acetate $=$ 15:1). ${ }^{1} \mathrm{H}$ NMR $\left(400 \mathrm{MHz}, \mathrm{CDCl}_{3}\right) \delta$ 7.59-7.56 (t, $\left.J=6.2 \mathrm{~Hz}, 1 \mathrm{H}\right), 7.48-7.46(\mathrm{~m}, 2 \mathrm{H}), 7.37-7.35$ $(\mathrm{m}, 3 \mathrm{H}), 5.55(\mathrm{~s}, 1 \mathrm{H}), 4.27-4.14(\mathrm{~m}, 2 \mathrm{H}), 2.21-2.16(\mathrm{~m}, 2 \mathrm{H}), 1.55-1.49(\mathrm{q}, J=7.4 \mathrm{~Hz}, 2 \mathrm{H})$, $1.25-1.21(\mathrm{t}, J=14.2 \mathrm{~Hz}, 3 \mathrm{H}), 0.96-0.92(\mathrm{t}, J=7.4 \mathrm{~Hz}, 3 \mathrm{H}) \mathrm{ppm} ;{ }^{13} \mathrm{C} \mathrm{NMR}\left(100 \mathrm{MHz}, \mathrm{CDCl}_{3}\right) \delta$ $170.7,153.1,135.0,128.9,128.6,127.6,83.0,61.2,31.3,19.9,14.1,13.5$ ppm; IR (Neat) $v_{\max }$ 2962, 2933, 1753, 1455, 1262, 1204, 1177, 1066, 922, $697 \mathrm{~cm}^{-1}$; HRMS (ESI) m/z: $[\mathrm{M}+\mathrm{H}]^{+}$Calcd for $\mathrm{C}_{14} \mathrm{H}_{20} \mathrm{NO}_{3} 250.1438$; Found 250.1439.

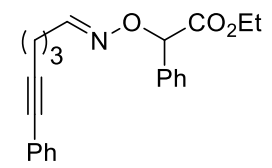

Ethyl (E)-2-phenyl-2-(((6-phenylhex-5-yn-1-ylidene)amino)oxy)acetate (4k). According to procedure B, 4k was obtained from 6-phenylhex-5-ynal oxime (37.4 mg, $0.2 \mathrm{mmol}$ ) and ethyl 2-diazo-2-phenylacetate (45.6 mg, $0.24 \mathrm{mmol})$ as light yellow oil (42.5 mg, 61\% yield, eluent: petroleum ether/ethyl acetate $=15: 1) .{ }^{1} \mathrm{H} \mathrm{NMR}\left(400 \mathrm{MHz}, \mathrm{CDCl}_{3}\right) \delta 7.65-7.62(\mathrm{t}, J=5.9 \mathrm{~Hz}, 1 \mathrm{H})$, 7.48-7.46 (m, 2H), 7.39-7.35 (m, 5H), 7.27-7.26 (m, 3H), $5.56(\mathrm{~s}, 1 \mathrm{H}), 4.28-4.14(\mathrm{~m}, 2 \mathrm{H})$, 2.48-2.45 (t, $J=7.0 \mathrm{~Hz}, 2 \mathrm{H}), 2.43-2.37(\mathrm{~m}, 2 \mathrm{H}), 1.85-1.78(\mathrm{~m}, 2 \mathrm{H}), 1.25-1.22(\mathrm{t}, J=7.1 \mathrm{~Hz}, 3 \mathrm{H})$ ppm; ${ }^{13} \mathrm{C} \mathrm{NMR}\left(100 \mathrm{MHz}, \mathrm{CDCl}_{3}\right) \delta 170.7,152.2,134.9,131.6,128.9,128.6,128.2,127.6,123.7$, 89.0, 83.1, 81.4, 61.2, 28.6, 25.5, 18.8, 14.1 ppm; IR (Neat) $v_{\max } 3063,2933,1984,1750,1489$, 1454, 1262, 1205, 1178, 1063, 1027, 916, 757, $693 \mathrm{~cm}^{-1}$; HRMS (ESI) m/z: $[\mathrm{M}+\mathrm{H}]^{+}$Calcd for $\mathrm{C}_{22} \mathrm{H}_{24} \mathrm{NO}_{3} 350.1751$; Found 350.1754 .<smiles>CCOC(=O)C(O/N=C/C(C)(C)C)c1ccccc1</smiles>

Ethyl (E)-2-(((2,2-dimethylpropylidene)amino)oxy)-2-phenylacetate (4I). According to procedure B, 41 was obtained from pivalaldehyde oxime $(20.4 \mathrm{mg}, 0.2 \mathrm{mmol})$ and ethyl 2-diazo-2-phenylacetate $(45.6 \mathrm{mg}, 0.24 \mathrm{mmol})$ as light yellow oil $(29.5 \mathrm{mg}, 56 \%$ yield, eluent: 
petroleum ether/ethyl acetate $=15: 1) .{ }^{1} \mathrm{H}$ NMR $\left(400 \mathrm{MHz}, \mathrm{CDCl}_{3}\right) \delta 7.50(\mathrm{~s}, 1 \mathrm{H}), 7.49-7.46(\mathrm{~m}$, 2H), 7.39-7.34 (m, 3H), $5.54(\mathrm{~s}, 1 \mathrm{H}), 4.24-4.18(\mathrm{~m}, 2 \mathrm{H}), 1.26-1.22(\mathrm{t}, J=7.1 \mathrm{~Hz}, 3 \mathrm{H}), 1.09(\mathrm{~s}, 9 \mathrm{H})$ ppm; ${ }^{13} \mathrm{C} \mathrm{NMR}\left(100 \mathrm{MHz}, \mathrm{CDCl}_{3}\right) \delta 170.8,160.2,135.0,128.8,128.6,127.6,83.1,61.1,33.8$, 27.5, 14.2 ppm; IR (Neat) $v_{\max } 2966,1826,1753,1455,1367,1259,1203,1177,1095,1028,906$, $697 \mathrm{~cm}^{-1}$; HRMS (ESI) m/z: [M+H] $]^{+}$Calcd for $\mathrm{C}_{15} \mathrm{H}_{22} \mathrm{NO}_{3}$ 264.1594; Found 264.1595.

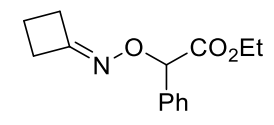

Ethyl 2-((cyclobutylideneamino)oxy)-2-phenylacetate (4m). According to procedure B, $4 \mathrm{~m}$ was obtained from cyclobutanone oxime $(17.1 \mathrm{mg}, 0.2 \mathrm{mmol})$ and ethyl 2-diazo-2-phenylacetate (45.6 $\mathrm{mg}, 0.24 \mathrm{mmol})$ as light yellow oil $(29.6 \mathrm{mg}, 60 \%$ yield, eluent: petroleum ether/ethyl acetate $=$ 15:1). ${ }^{1} \mathrm{H}$ NMR (400 MHz, $\left.\mathrm{CDCl}_{3}\right) \delta$ 7.56-7.54 (m, 2H), 7.46-7.39 (m, 3H), $5.57(\mathrm{~s}, 1 \mathrm{H})$, 4.32-4.21 (m, 2H), 3.17-3.13 (m, 1H), 3.09-2.98 (m, 3H), 2.13-2.05 (m, 2H), 1.32-1.29 (t, $J=7.1$ $\mathrm{Hz}, 3 \mathrm{H}) \mathrm{ppm} ;{ }^{13} \mathrm{C} \mathrm{NMR}\left(100 \mathrm{MHz}, \mathrm{CDCl}_{3}\right) \delta 170.9,161.5,135.2,128.8,128.5,127.6,83.0,61.1$, 31.7, 31.5, 14.5, 14.1 ppm; IR (Neat) $v_{\max } 2963,2929,1781,1750,1732,1454,1368,1260,1203$, 1176, 1062, 1024, 896, 797, 696, $622 \mathrm{~cm}^{-1}$; HRMS (ESI) $m / z:[\mathrm{M}+\mathrm{H}]^{+}$Calcd for $\mathrm{C}_{14} \mathrm{H}_{18} \mathrm{NO}_{3}$ 248.1281; Found 248.1284.

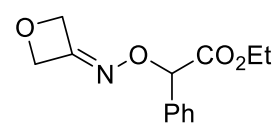

Ethyl 2-((oxetan-3-ylideneamino)oxy)-2-phenylacetate (4n). According to procedure B, 4n was obtained from oxetan-3-one oxime (17.4 mg, $0.2 \mathrm{mmol})$ and ethyl 2-diazo-2-phenylacetate (45.6 $\mathrm{mg}, 0.24 \mathrm{mmol})$ as light yellow oil $(27.5 \mathrm{mg}, 55 \%$ yield, eluent: petroleum ether/ethyl acetate $=$ 15:1). ${ }^{1} \mathrm{H}$ NMR (400 MHz, DMSO- $\left.d_{6}\right) \delta$ 7.44-7.39 (m, 5H), $5.60(\mathrm{~s}, 1 \mathrm{H}), 5.30-5.25(\mathrm{~m}, 4 \mathrm{H})$, 4.17-4.10 (m, 2H), 1.16-1.13 (t, $J=7.1 \mathrm{~Hz}, 3 \mathrm{H}) \mathrm{ppm} ;{ }^{13} \mathrm{C}$ NMR (100 MHz, DMSO-d 6 ) $\delta 170.2$, $156.3,134.8,129.7,129.2,128.2,83.1,78.6,78.2,61.4,14.4$ ppm; IR (Neat) $v_{\max } 2981,2930$, 2107, 1749, 1455, 1263, 1206, 1179, 1061, 955, 906, 859, 733, 697, $622 \mathrm{~cm}^{-1}$; HRMS (ESI) $\mathrm{m} / z$ : $[\mathrm{M}+\mathrm{Na}]^{+}$Calcd for $\mathrm{C}_{13} \mathrm{H}_{15} \mathrm{NNaO}_{4}$ 272.0893; Found 272.0893.<smiles>CCOC(ON=C1CCN(C(=O)c2ccccc2)CC1)c1ccccc1</smiles>

tert-Butyl 4-((2-ethoxy-2-oxo-1-phenylethoxy)imino)piperidine-1-carboxylate (40). According 
to procedure B, $4 \mathbf{0}$ was obtained from tert-butyl 4-(hydroxyimino)piperidine-1-carboxylate (42.8 $\mathrm{mg}, 0.2 \mathrm{mmol}$ ) and ethyl 2-diazo-2-phenylacetate $(45.6 \mathrm{mg}, 0.24 \mathrm{mmol})$ as light yellow oil (31.7 mg, $42 \%$ yield, eluent: petroleum ether/ethyl acetate $=10: 1) .{ }^{1} \mathrm{H}$ NMR $\left(400 \mathrm{MHz}, \mathrm{CDCl}_{3}\right) \delta$ 7.63-7.61 (m, 2H), 7.39-7.35 (m, 2H), 7.32-7.29 (m, 1H), 4.34-4.24 (m, 1H), 4.22-4.16 (m, 2H), $3.88(\mathrm{~s}, 2 \mathrm{H}), 3.52-3.47(\mathrm{~m}, 1 \mathrm{H}), 3.01-2.95(\mathrm{~m}, 2 \mathrm{H}), 2.54-2.41(\mathrm{~m}, 3 \mathrm{H}), 1.37(\mathrm{~s}, 9 \mathrm{H}), 1.27-1.23(\mathrm{t}, J$ $=7.1 \mathrm{~Hz}, 3 \mathrm{H}) \mathrm{ppm} ;{ }^{13} \mathrm{C} \mathrm{NMR}\left(100 \mathrm{MHz}, \mathrm{CDCl}_{3}\right) \delta 207.9,173.8,154.3,128.5,128.5,128.2$, 125.3, 80.5, 77.2, 62.5, 43.3, 41.4, 28.2, 13.9 ppm; IR (Neat) $v_{\max } 3492,2976,2929,1732,1697$, 1419, 1366, 1239, 1162, 1111, 732, $517 \mathrm{~cm}^{-1}$; HRMS (ESI) m/z: $[\mathrm{M}+\mathrm{Na}]^{+}$Calcd for $\mathrm{C}_{20} \mathrm{H}_{28} \mathrm{~N}_{2} \mathrm{NaO}_{5}$ 399.1890; Found 399.1894.

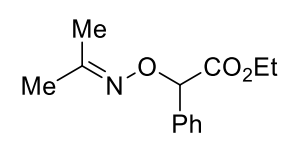

Ethyl 2-phenyl-2-((propan-2-ylideneamino)oxy)acetate (4p). According to procedure B, $4 p$ was obtained from propan-2-one oxime (15.3 mg, $0.2 \mathrm{mmol})$ and ethyl 2-diazo-2-phenylacetate (45.6 $\mathrm{mg}, 0.24 \mathrm{mmol})$ as light yellow oil $(24.4 \mathrm{mg}, 52 \%$ yield, eluent: petroleum ether/ethyl acetate $=$ 15:1). ${ }^{1} \mathrm{H}$ NMR (400 MHz, $\left.\mathrm{CDCl}_{3}\right) \delta$ 7.50-7.47 (m, 2H), 7.39-7.34 (m, 3H), $5.53(\mathrm{~s}, 1 \mathrm{H})$, 4.23-4.14 (m, 2H), 1.98 (s, 3H), $1.89(\mathrm{~s}, 3 \mathrm{H}), 1.24-1.20(\mathrm{t}, J=7.1 \mathrm{~Hz}, 3 \mathrm{H}) \mathrm{ppm} ;{ }^{13} \mathrm{C}$ NMR $(100$ $\left.\mathrm{MHz}, \mathrm{CDCl}_{3}\right) \delta 171.0,157.2,135.4,128.7,128.5,127.5,82.8,61.0,21.8,16.1,14.1 \mathrm{ppm}$; IR (Neat) $v_{\max } 2980,2924,1720,1445,1260,1218,1025,745,698 \mathrm{~cm}^{-1}$; HRMS (ESI) $m / z:[\mathrm{M}+\mathrm{H}]^{+}$ Calcd for $\mathrm{C}_{13} \mathrm{H}_{18} \mathrm{NO}_{3} 236.1281$; Found 236.1278 .<smiles>CCOC(ON=C(C)c1ccccc1)c1ccccc1</smiles>

Ethyl (E)-2-phenyl-2-(((1-phenylethylidene)amino)oxy)acetatee $(4 q)^{5}$. According to procedure B, 4q was obtained from 1-phenylethan-1-one oxime (27.3 $\mathrm{mg}, 0.2 \mathrm{mmol})$ and ethyl 2-diazo-2-phenylacetate $(45.6 \mathrm{mg}, 0.24 \mathrm{mmol})$ as light yellow oil $(46.9 \mathrm{mg}, 79 \%$ yield, eluent: petroleum ether/ethyl acetate $=15: 1) .{ }^{1} \mathrm{H}$ NMR $\left(400 \mathrm{MHz}, \mathrm{CDCl}_{3}\right) \delta$ 7.65-7.63 $(\mathrm{m}, 2 \mathrm{H})$, 7.56-7.53 (m, 2H), 7.42-7.34 (m, 6H), $5.72(\mathrm{~s}, 1 \mathrm{H}), 4.25-4.16(\mathrm{~m}, 2 \mathrm{H}), 2.36(\mathrm{~s}, 3 \mathrm{H}), 1.25-1.21$ (t, $J=7.1 \mathrm{~Hz}$, $3 \mathrm{H}) \mathrm{ppm} ;{ }^{13} \mathrm{C} \mathrm{NMR}\left(100 \mathrm{MHz}, \mathrm{CDCl}_{3}\right) \delta 170.8,156.8,136.1,135.2,129.3,128.8,128.6,128.3$, 127.6, 126.3, 83.6, 61.1, 14.1, 13.2 ppm; IR (Neat) $v_{\max } 3062,2981,2933,1751,1496,1454,1369$, 1262, 1204, 1177, 1061, 1027, 924, 761, 694, $657 \mathrm{~cm}^{-1}$; HRMS (ESI) $m / z:[\mathrm{M}+\mathrm{H}]^{+}$Calcd for 
$\mathrm{C}_{18} \mathrm{H}_{20} \mathrm{NO}_{3} 298.1438$; Found 298.1442.<smiles>CCCOC(=O)C(OC=NO)c1ccccc1</smiles>

2-(Trimethylsilyl)ethyl (E)-2-((benzylideneamino)oxy)-2-phenylacetate (4r). According to procedure B, 4r was obtained from benzaldehyde oxime $(24.2 \mathrm{mg}, 0.2 \mathrm{mmol})$ and 2-(trimethylsilyl)ethyl 2-diazo-2-phenylacetate $(63.3 \mathrm{mg}, 0.24 \mathrm{mmol})$ as light yellow oil (54.1 $\mathrm{mg}$, $76 \%$ yield, eluent: petroleum ether/ethyl acetate $=15: 1) .{ }^{1} \mathrm{H}$ NMR $\left(400 \mathrm{MHz}, \mathrm{CDCl}_{3}\right) \delta 8.29(\mathrm{~s}$, 1H), 7.62-7.60 (m, 2H), 7.57-7.55 (m, 2H), 7.43-7.39 (m, 6H), $5.72(\mathrm{~s}, 1 \mathrm{H}), 4.37-4.22(\mathrm{~m}, 2 \mathrm{H})$, 1.04-1.00 (m, 2H), 0.03 (s, 9H) ppm; ${ }^{13} \mathrm{C}$ NMR $\left(100 \mathrm{MHz}, \mathrm{CDCl}_{3}\right) \delta 170.7,150.5,134.8,131.7$, $130.1,129.0,128.6,128.6,127.7,127.3,83.9,63.7,17.2,-1.6$ ppm; IR (Neat) $v_{\max } 3064,2952$, $1749,1447,1249,1207,1168,1064,934,834,691,509 \mathrm{~cm}^{-1}$; HRMS (ESI) $m / z:[\mathrm{M}+\mathrm{Na}]^{+}$Calcd for $\mathrm{C}_{20} \mathrm{H}_{25} \mathrm{NNaO}_{3} \mathrm{Si}$ 378.1496; Found 378.1499.<smiles>CCCOC(=O)C(ON=Cc1ccccc1)c1ccccc1</smiles>

2-(Methylthio)ethyl (E)-2-((benzylideneamino)oxy)-2-phenylacetate (4s). According to procedure B, 4s was obtained from benzaldehyde oxime $(24.2 \mathrm{mg}, 0.2 \mathrm{mmol})$ and 2-(methylthio)ethyl 2-diazo-2-phenylacetate $(57.4 \mathrm{mg}, 0.24 \mathrm{mmol})$ as light yellow oil (44.9 $\mathrm{mg}$, $68 \%$ yield, eluent: petroleum ether/ethyl acetate $=15: 1) .{ }^{1} \mathrm{H} \mathrm{NMR}\left(400 \mathrm{MHz}, \mathrm{CDCl}_{3}\right) \delta 8.26(\mathrm{~s}$, 1H), 7.59-7.57 (m, 2H), 7.54-7.52 (m, 2H), 7.43-7.35 (m, 6H), $5.73(\mathrm{~s}, 1 \mathrm{H}), 4.39-4.29(\mathrm{~m}, 2 \mathrm{H})$, 2.71-2.67 (m, 2H), 2.08 (s, 3H) ppm; ${ }^{13} \mathrm{C}$ NMR (100 MHz, $\left.\mathrm{CDCl}_{3}\right) \delta 170.4,150.7,134.5,131.6$, 130.2, 129.1, 128.7, 128.7, 127.7, 127.4, 83.7, 64.0, 32.2, 15.7 ppm; IR (Neat) $v_{\max } 3062,2919$, 1982, 1751, 1447, 1260, 1203, 1169, 1066, 934, 755, 692, 655, $509 \mathrm{~cm}^{-1}$; HRMS (ESI) m/z: $[\mathrm{M}+\mathrm{Na}]^{+}$Calcd for $\mathrm{C}_{18} \mathrm{H}_{19} \mathrm{NNaO}_{3} \mathrm{~S}$ 352.0978; Found 352.0980.<smiles>O=C(OCC1CCCO1)C(ON=Cc1ccccc1)c1ccccc1</smiles>

(Tetrahydrofuran-2-yl)methyl

(E)-2-((benzylideneamino)oxy)-2-phenylacetate

(4t).

According to procedure B, $4 \mathbf{t}$ was obtained from benzaldehyde oxime $(24.2 \mathrm{mg}, 0.2 \mathrm{mmol})$ and 2-diazo-2-phenylacetate $(59.2 \mathrm{mg}, 0.24 \mathrm{mmol})$ as light yellow oil $(48.1 \mathrm{mg}, 71 \%$ yield, eluent: petroleum ether/ethyl acetate $=10: 1) .{ }^{1} \mathrm{H}$ NMR $\left(400 \mathrm{MHz}, \mathrm{CDCl}_{3}\right) \delta 8.26(\mathrm{~s}, 1 \mathrm{H}), 7.59-7.53(\mathrm{~m}$, 
4H), 7.42-7.34 (m, 6H), $5.76(\mathrm{~s}, 1 \mathrm{H}), 4.27-4.12(\mathrm{~m}, 2 \mathrm{H})$ 4.11-4.04 (m, 1H), 3.81-3.69 (m, 2H), $1.95-1.73(\mathrm{~m}, 3 \mathrm{H}), 1.61-1.48(\mathrm{~m}, 1 \mathrm{H}) \mathrm{ppm} ;{ }^{13} \mathrm{C} \mathrm{NMR}\left(100 \mathrm{MHz}, \mathrm{CDCl}_{3}\right) \delta 170.5,150.6,134.6$, 131.6, 130.2, 129.0, 128.6, 128.6, 127.7, 127.3, 83.6, 76.3, 68.4, 66.9, 27.9, 25.6 ppm; IR (Neat) $v_{\max } 3063,2951,2873,1753,1447,1268,1205,1173,1088,1067,940,756,694 \mathrm{~cm}^{-1}$; HRMS (ESI) $m / z:[\mathrm{M}+\mathrm{H}]^{+}$Calcd for $\mathrm{C}_{20} \mathrm{H}_{22} \mathrm{NO}_{4} 340.1543$; Found 340.1547 .

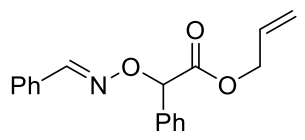

Allyl (E)-2-((benzylideneamino)oxy)-2-phenylacetate (4u). According to procedure B, $4 \mathbf{u}$ was obtained from benzaldehyde oxime $(24.2 \mathrm{mg}, 0.2 \mathrm{mmol})$ and allyl 2-diazo-2-phenylacetate (50.4 $\mathrm{mg}, 0.24 \mathrm{mmol})$ as light yellow oil $(47.7 \mathrm{mg}, 81 \%$ yield, eluent: petroleum ether/ethyl acetate $=$ 15:1). ${ }^{1} \mathrm{H}$ NMR $\left(400 \mathrm{MHz}, \mathrm{CDCl}_{3}\right) \delta 8.27(\mathrm{~s}, 1 \mathrm{H}), 7.59-7.57(\mathrm{~m}, 2 \mathrm{H}), 7.55-7.52(\mathrm{~m}, 2 \mathrm{H})$, 7.41-7.35 (m, 6H), 5.91-5.82 (m, 1H), $5.75(\mathrm{~s}, 1 \mathrm{H})$, 5.27-5.16 (m, 2H), 4.68-4.66 (m, 2H) ppm; ${ }^{13} \mathrm{C}$ NMR $\left(100 \mathrm{MHz}, \mathrm{CDCl}_{3}\right) \delta 170.2,150.7,134.7,131.6,130.2,129.1,128.7,128.6,128.6$, 127.7, 127.4, 118.3, 83.7, 65.6 ppm; IR (Neat) $v_{\max } 3064,2934,1751,1447,1268,1201,1170$, 1090, 1065, 938, 731, 691, $508 \mathrm{~cm}^{-1}$; HRMS (ESI) $\mathrm{m} / z$ : $[\mathrm{M}+\mathrm{H}]^{+}$Calcd for $\mathrm{C}_{18} \mathrm{H}_{18} \mathrm{NO}_{3} 296.1281$; Found 296.1285 .<smiles>CC#CCOC(=O)C(ON=Cc1ccccc1)c1ccccc1</smiles>

But-2-yn-1-yl (E)-2-((benzylideneamino)oxy)-2-phenylacetate (4v). According to procedure B, 4v was obtained from benzaldehyde oxime $(24.2 \mathrm{mg}, 0.2 \mathrm{mmol})$ and but-2-yn-1-yl 2-diazo-2-phenylacetate $(51.4 \mathrm{mg}, 0.24 \mathrm{mmol})$ as light yellow oil $(51.0 \mathrm{mg}, 83 \%$ yield, eluent: petroleum ether/ethyl acetate $=15: 1) .{ }^{1} \mathrm{H}$ NMR $\left(400 \mathrm{MHz}, \mathrm{CDCl}_{3}\right) \delta 8.26(\mathrm{~s}, 1 \mathrm{H}), 7.59-7.52(\mathrm{~m}$, 4H), 7.40-7.35 (m, 6H), $5.75(\mathrm{~s}, 1 \mathrm{H}), 4.80-4.66(\mathrm{~m}, 2 \mathrm{H}), 1.80-1.79(\mathrm{t}, J=2.4 \mathrm{~Hz}, 3 \mathrm{H}) \mathrm{ppm} ;{ }^{13} \mathrm{C}$ $\operatorname{NMR}\left(100 \mathrm{MHz}, \mathrm{CDCl}_{3}\right) \delta 170.0,150.7,134.3,131.6,130.2,129.1,128.7,128.6,127.8,127.4$ 83.7, 83.6, 72.6, 53.6, 3.6 ppm; IR (Neat) $v_{\max }$ 3063, 2920, 2242, 1986, 1758, 1447, 1265, 1166, 1067, 936, 756, 694, $510 \mathrm{~cm}^{-1}$; HRMS (ESI) $\mathrm{m} / \mathrm{z}$ : $[\mathrm{M}+\mathrm{H}]^{+}$Calcd for $\mathrm{C}_{19} \mathrm{H}_{18} \mathrm{NO}_{3}$ 308.1281; Found 308.1286 . 
<smiles>O=C(OCc1ccccc1)C(OC=Nc1ccccc1)c1ccccc1</smiles>

Benzyl (E)-2-((benzylideneamino)oxy)-2-phenylacetate (4w). According to procedure B, 4 w was obtained from benzaldehyde oxime (24.2 $\mathrm{mg}, 0.2 \mathrm{mmol})$ and allyl benzyl 2-diazo-2-phenylacetate $(60.5 \mathrm{mg}, 0.24 \mathrm{mmol})$ as light yellow oil $(55.2 \mathrm{mg}, 80 \%$ yield, eluent: petroleum ether/ethyl acetate $=15: 1) .{ }^{1} \mathrm{H}$ NMR $\left(400 \mathrm{MHz}, \mathrm{CDCl}_{3}\right) \delta 8.25(\mathrm{~s}, 1 \mathrm{H}), 7.56-7.51(\mathrm{~m}$, 4H), 7.38-7.33 (m, 6H), 7.26-7.25 (m, 5H), 5.78 (s, 1H), 5.24-5.17 (m, 2H) ppm; ${ }^{13} \mathrm{C}$ NMR (100 $\left.\mathrm{MHz}, \mathrm{CDCl}_{3}\right) \delta 170.4,150.6,135.5,134.5,131.5,130.2,129.1,128.6,128.6,128.4,128.1,127.8$, 127.7, 127.3, 83.7, 66.7 ppm; IR (Neat) $v_{\max }$ 3031, 2928, 1802, 1773, 1591, 1491, 1462, 1232 , 1193, 1162, 1066, 935, 754, $691 \mathrm{~cm}^{-1}$; HRMS (ESI) $\mathrm{m} / z$ : $[\mathrm{M}+\mathrm{H}]^{+}$Calcd for $\mathrm{C}_{22} \mathrm{H}_{20} \mathrm{NO}_{3} 346.1438$; Found 346.1447.

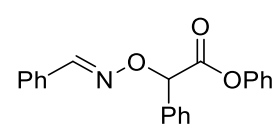

Phenyl (E)-2-((benzylideneamino)oxy)-2-phenylacetate (4x). According to procedure B, $4 x$ was obtained from benzaldehyde oxime $(24.2 \mathrm{mg}, 0.2 \mathrm{mmol})$ and allyl phenyl 2-diazo-2-phenylacetate (57.1 mg, $0.24 \mathrm{mmol})$ as light yellow oil $(50.9 \mathrm{mg}, 77 \%$ yield, eluent: petroleum ether/ethyl acetate $=15: 1) .{ }^{1} \mathrm{H}$ NMR $\left(400 \mathrm{MHz}, \mathrm{CDCl}_{3}\right) \delta 8.31(\mathrm{~s}, 1 \mathrm{H}), 7.65-7.61(\mathrm{~m}, 4 \mathrm{H}), 7.45-7.32(\mathrm{~m}, 8 \mathrm{H})$, 7.22-7.17 (m, 2H), 7.06-7.03 (m, 1H), $5.94(\mathrm{~s}, 1 \mathrm{H}) \mathrm{ppm} ;{ }^{13} \mathrm{C} \mathrm{NMR}\left(100 \mathrm{MHz}, \mathrm{CDCl}_{3}\right) \delta 169.1$, $150.9,134.2,131.5,130.3,129.4,129.3,128.9,128.7,127.9,127.4,126.0,121.3,83.8$ ppm; IR (Neat) $v_{\max } 3063,2929,1773,1618,1592,1491,1455,1232,1193,1162,1066,1025,935,901$, 754, 692, $523 \mathrm{~cm}^{-1}$; HRMS (ESI) m/z: [M+H] $]^{+}$Calcd for $\mathrm{C}_{21} \mathrm{H}_{18} \mathrm{NO}_{3}$ 332.1281; Found 332.1282.

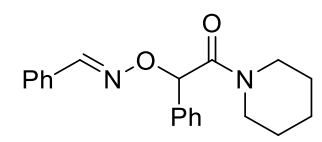

(E)-Benzaldehyde $O$-(2-oxo-1-phenyl-2-(piperidin-1-yl)ethyl) oxime (4y). According to procedure B, 4y was obtained from benzaldehyde oxime $(24.2 \mathrm{mg}, 0.2 \mathrm{mmol})$ and 2-diazo-2-phenyl-1-(piperidin-1-yl)ethan-1-one $(55.1 \mathrm{mg}, 0.24 \mathrm{mmol})$ as light yellow oil (51.5 mg, $80 \%$ yield, eluent: petroleum ether/ethyl acetate $=15: 1) .{ }^{1} \mathrm{H} \mathrm{NMR}\left(400 \mathrm{MHz}, \mathrm{CDCl}_{3}\right) \delta 8.26(\mathrm{~s}$, 1H), 7.59-7.57 (m, 2H), 7.52-7.50 (m, 2H), 7.42-7.33 (m, 6H), $6.04(\mathrm{~s}, 1 \mathrm{H}), 3.72-3.67(\mathrm{~m}, 1 \mathrm{H})$, 3.54-3.48 (m, 1H), 3.40-3.38 (m, 2H), 1.55-1.53 (m, 3H), 1.49-1.46 (m, 1H), 1.41-1.35 (m, 1H), 
$1.20-1.15(\mathrm{~m}, 1 \mathrm{H}) \mathrm{ppm} ;{ }^{13} \mathrm{C} \mathrm{NMR}\left(100 \mathrm{MHz}, \mathrm{CDCl}_{3}\right) \delta 167.8,150.2,135.5,131.8,130.0,128.7$, $128.7,128.6,127.8,127.3,83.5,46.2,43.3,25.8,25.5,24.4$ ppm; IR (Neat) $v_{\max } 3478,2935,2855$, $1745,1651,1444,1269,1060,1009,947,756,694,509 \mathrm{~cm}^{-1}$; HRMS (ESI) $m / z:[\mathrm{M}+\mathrm{H}]^{+}$Calcd for $\mathrm{C}_{20} \mathrm{H}_{23} \mathrm{~N}_{2} \mathrm{O}_{2}$ 323.1754; Found 323.1757.

$$
\mathrm{Ph} \widehat{\mathrm{N}^{-} \mathrm{O}} \underset{\text { 2-naphthyl }}{\mathrm{CO}_{2} \mathrm{Et}}
$$

Ethyl (E)-2-((benzylideneamino)oxy)-2-(naphthalen-1-yl)acetate (4z). According to procedure B, $4 \mathbf{z}$ was obtained from benzaldehyde oxime $(24.2 \mathrm{mg}, 0.2 \mathrm{mmol})$ and ethyl 2-diazo-2-(naphthalen-1-yl)acetate (57.6 mg, $0.24 \mathrm{mmol})$ as light yellow oil $(49.5 \mathrm{mg}, 74 \%$ yield, eluent: petroleum ether/ethyl acetate $=15: 1) .{ }^{1} \mathrm{H}$ NMR $\left(400 \mathrm{MHz}, \mathrm{CDCl}_{3}\right) \delta 8.31-8.29(\mathrm{~d}, J=8.6$ $\mathrm{Hz}, 1 \mathrm{H}), 8.28(\mathrm{~s}, 1 \mathrm{H}), 7.89-7.88(\mathrm{~d}, J=8.4 \mathrm{~Hz}, 2 \mathrm{H}), 7.66-7.65(\mathrm{~m}, 1 \mathrm{H}), 7.61-7.47(\mathrm{~m}, 5 \mathrm{H})$, 7.39-7.36 (m, 3H), $6.42(\mathrm{~s}, 1 \mathrm{H}), 4.31-4.18(\mathrm{~m}, 2 \mathrm{H}), 1.23-1.20(\mathrm{t}, J=7.1 \mathrm{~Hz}, 3 \mathrm{H}) \mathrm{ppm} ;{ }^{13} \mathrm{C}$ NMR $\left(100 \mathrm{MHz}, \mathrm{CDCl}_{3}\right) \delta 170.8,150.6,133.9,131.7,131.3,130.9,130.2,129.8,128.7,128.7,127.4$, 126.7, 126.6, 125.9, 125.2, 124.0, 81.7, 61.4, 14.1ppm; IR (Neat) $v_{\max } 3052,2980,2930,1817$, 1749, 1446, 1194, 1092, 941, 776, $693 \mathrm{~cm}^{-1}$; HRMS (ESI) $\mathrm{m} / \mathrm{z}$ : $[\mathrm{M}+\mathrm{Na}]^{+}$Calcd for $\mathrm{C}_{21} \mathrm{H}_{19} \mathrm{NNaO}_{3}$ 356.1257; Found 356.1259.

\section{Gram scale reactions}

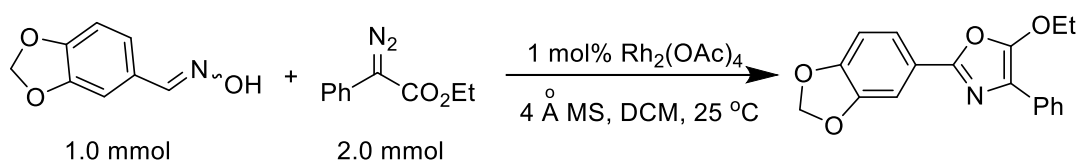

1b

2a

$3 \mathbf{b}$

Under $\mathrm{Ar}$ armosphere, to a $25 \mathrm{~mL}$ over-dried Schlenk tube charged with a stir bar, benzo $[d][1,3]$ dioxole-5-carbaldehyde oxime $\quad(165 \mathrm{mg}, \quad 1.0 \mathrm{mmol}, \quad 1.0 \quad$ equiv. $)$ ethyl 2-diazo-2-phenylacetate (380 mg, $2.0 \mathrm{mmol}, 2.0$ equiv.) and 4 Å molecular sieves (100 mg), was added $\mathrm{Rh}_{2}(\mathrm{OAc})_{4}(0.01 \mathrm{mmol}, 1 \mathrm{mmol} \%)$. The resultant solution was stirred at $\mathrm{rt}$ for 8 hours (monitored by TLC). After completion, a saturated aqueous $\mathrm{NH}_{4} \mathrm{Cl}$ solution $(15 \mathrm{~mL})$ was added and the reaction mixture was diluted with ethyl acetate $(15 \mathrm{~mL})$. The organic layer was separated and the aqueous layer was extracted with EtOAc $(15 \mathrm{~mL} \times 3)$. The combined extracts were washed with brine $(30 \mathrm{~mL})$, dried over anhydrous $\mathrm{Na}_{2} \mathrm{SO}_{4}$. After filtration, the filtrate was concentrated under vacuum and the obtained residue was purified by flash column chromatography on silica gel 
(petroleum ether and ethyl acetate as eluents, the ratio is 15:1) to afford the $\mathbf{3 b}$ as light yellow solid (210 mg, 68\% yield).

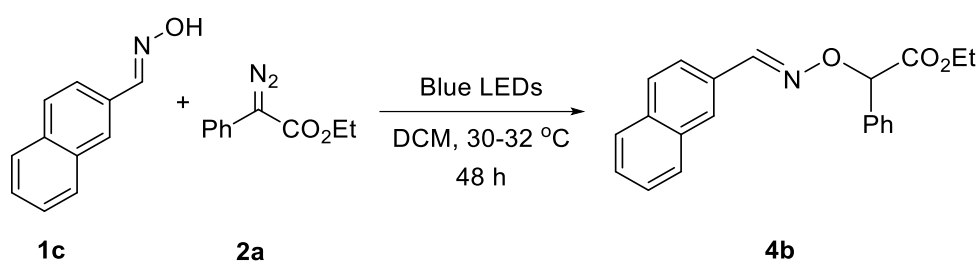

A $25 \mathrm{~mL}$ glass tube equipped with magnetic stir bar was charged with 2-naphthaldehyde oxime (171 mg, $1.0 \mathrm{mmol}, 1.0$ equiv.) ethyl 2-diazo-2-phenylacetate (206 mg, $1.2 \mathrm{mmol}, 1.2$ equiv) under argon atmosphere. The tube was evacuated and backfilled with argon atmosphere for 3 times. Degassed DCM $(10 \mathrm{~mL})$ was added by syringe under argon atmosphere. The mixture was then irradiated by $12 \mathrm{~W}$ blue LEDs and stirred at room temperature for 48 hours. Upon completion, the reaction mixture was concentrated under vacuum and the residue was purified by column chromatography (petroleum ether and ethyl acetate as eluents, the ratio is $15: 1$ ) to afford the $\mathbf{4 b}$ as light yellow solid (253 mg, 76\% yield).

\section{Control experiment}

\section{1 [3+2] Annulation of benzonitrile and 2a}

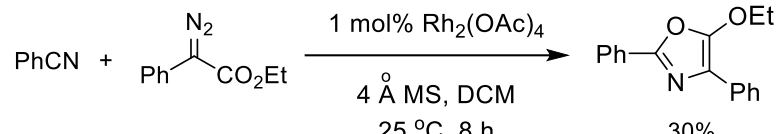

Under Ar atmosphere, to a $10 \mathrm{~mL}$ over-dried Schlenk tube charged with a stir bar, benzonitrile $(0.2$ mmol, 1.0 equiv.) $\alpha$-diazoester $\mathbf{2 a}(0.4$ mmol, 2.0 equiv.) and 4 Å molecular sieves (20 mg), was added $\mathrm{Rh}_{2}(\mathrm{OAc})_{4}(0.002 \mathrm{mmol}, 1 \mathrm{~mol} \%)$. The resultant solution was stirred at $\mathrm{rt}$ for 8 hours (monitored by TLC). After completion, a saturated aqueous $\mathrm{NH}_{4} \mathrm{Cl}$ solution $(5 \mathrm{~mL})$ was added and the reaction mixture was diluted with ethyl acetate $(5 \mathrm{~mL})$. The organic layer was separated and the aqueous layer was extracted with EtOAc $(5 \mathrm{~mL} \times 3)$. The combined extracts were washed with brine $(10 \mathrm{~mL})$, dried over anhydrous $\mathrm{Na}_{2} \mathrm{SO}_{4}$. After filtration, the filtrate was concentrated under vacuum and the obtained residue was purified by flash column chromatography on silica gel (petroleum ether and ethyl acetate as eluents, the ratio is from 10:1 to 4:1) to afford the oxazole.

\subsection{HRMS analyses of the reaction mixture}



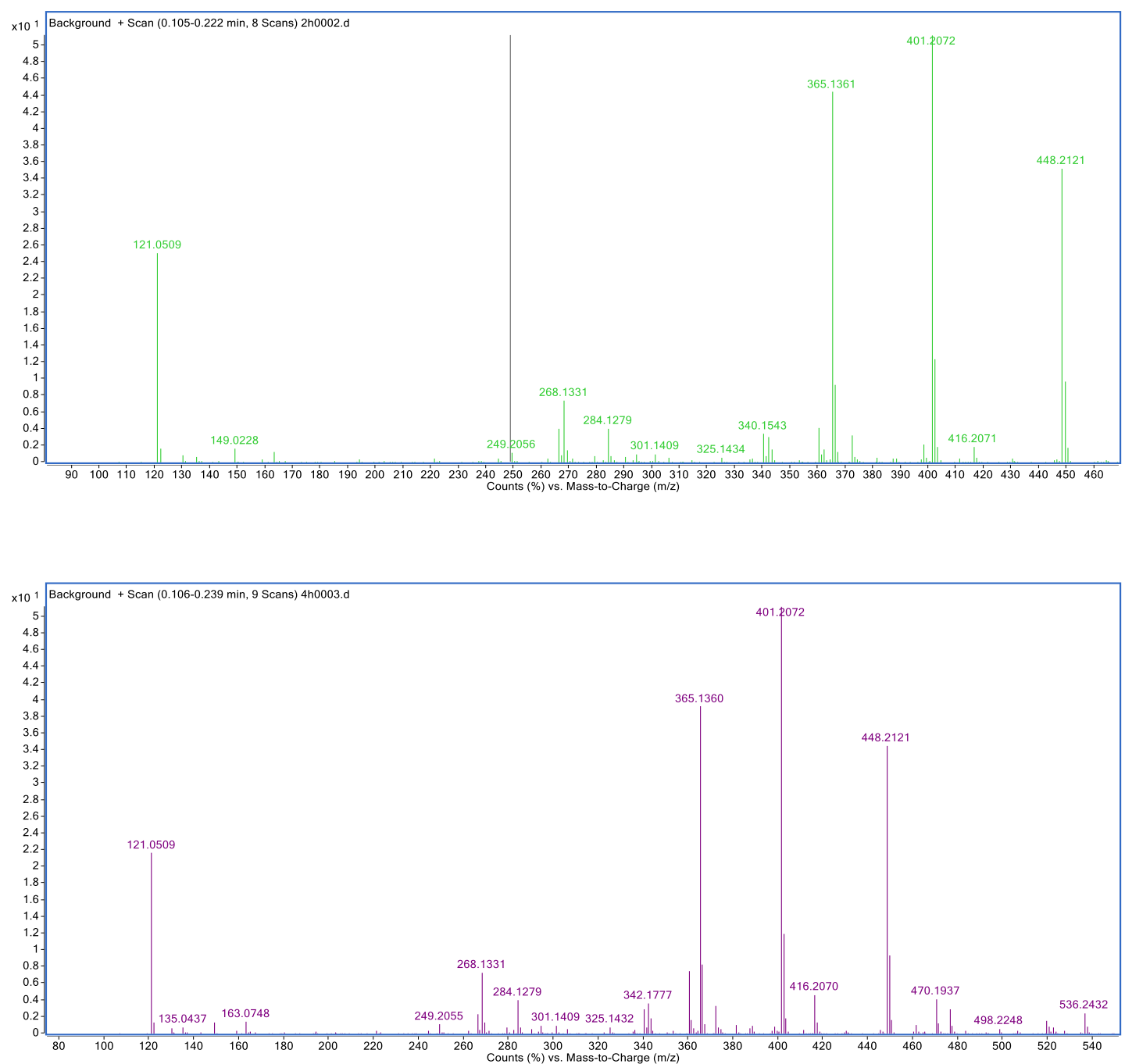

Figure S2. HRMS analyses of the reaction mixture (after $2 \mathrm{~h}$, top; after $4 \mathrm{~h}$, bottom)

\section{X-Ray crystallographic data of compounds $3 \mathrm{~b}$ and $4 \mathrm{~b}$}

Crystal 3a or 4a with the Volatilization Method: An amount of $15 \mathrm{mg}$ 3a or 4a was dissolved in DCM $(1 \mathrm{~mL})$ on the bright small reagent bottle $(5 \mathrm{~mL})$, which acted as good solvent, and a layer of $n$-hexane (1:1) was injected on the surface of DCM, and the cap is covered with a thin film, white crystals will be presented after several days. A suitable crystal was selected and mounted on a X-ray with Mo radiation $(\mathrm{a}=10.3244(13), 11.4076(15) \mathrm{c}=16.633(2))$ for cell determination and subsequent data collection at $296 \mathrm{~K}$. Using SHELXL-2014, the structurwas solved with the ShelXT $11^{6}$ structure solution program using Intrinsic Phasing and refined with the ShelXL12 refinemee package using Least Squares minimisation. The solved structures of $\mathbf{3 b}$ and $\mathbf{4 b}$ have 
been deposited in The Cambridge Crystallographic Data Centre (CCDC 2071710 for 3b and CCDC 2089154 for 4b). The single crystal analysis was carried out using Bruker Smart instrument.
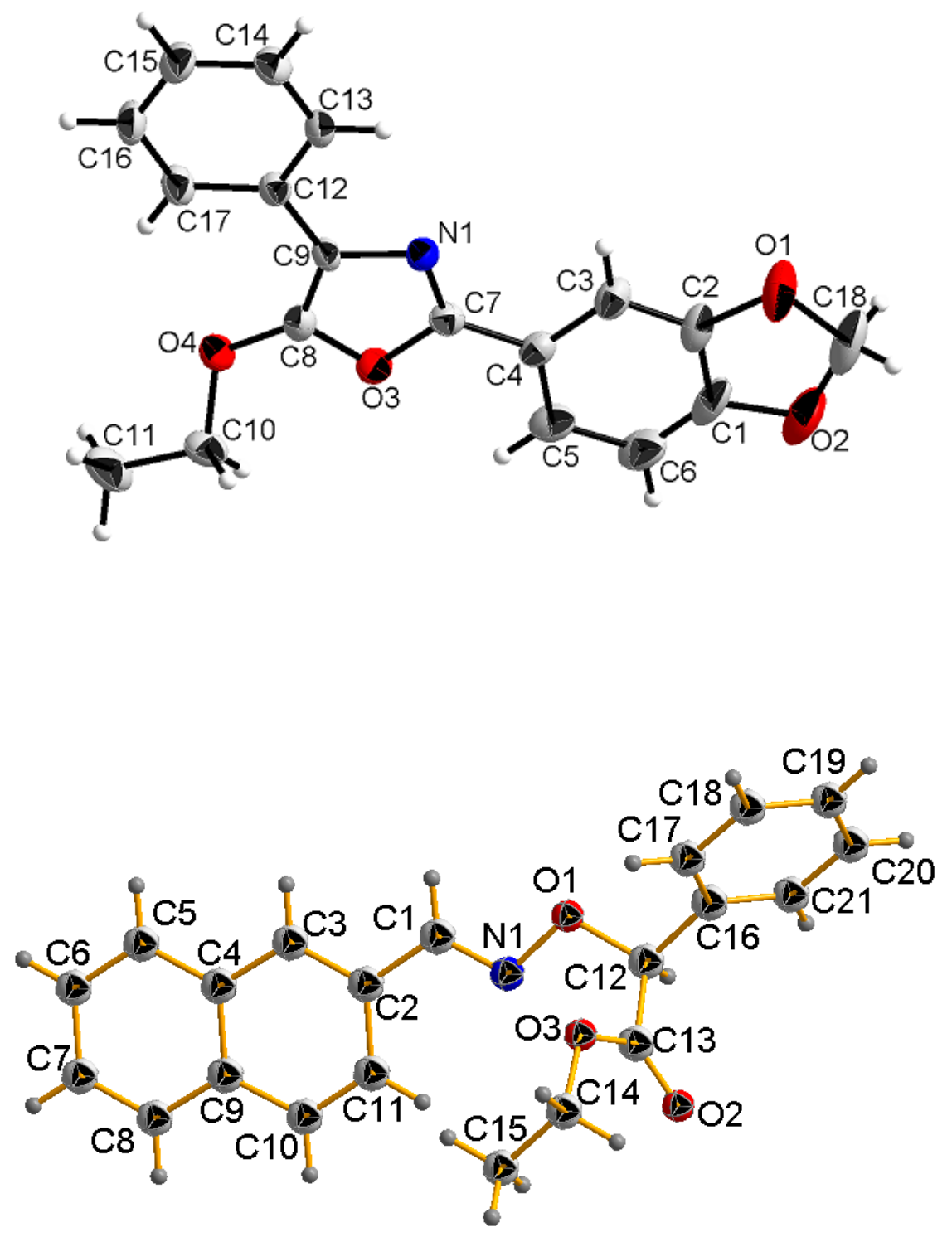

Figure S3. ORTEP diagram of compound $\mathbf{3 b}$ with ellipsoid shown at the $50 \%$ contour percent probability level (top) and ORTEP diagram of compound $\mathbf{4 b}$ with ellipsoid shown at the $50 \%$ contour percent probability level (bottom). 
Table S1. Crystal data and structure refinement for $3 \mathrm{~b}$.

Identification code

Empirical formula

Formula weight

Temperature

Wavelength

Crystal system

Space group

Unit cell dimensions

Volume

Z

Density (calculated)

Absorption coefficient

$\mathrm{F}(000)$

Crystal size

Theta range for data collection

Index ranges

Reflections collected

Independent reflections

Completeness to theta $=25.242^{\circ}$

Absorption correction

Refinement method

Data / restraints / parameters

Goodness-of-fit on $\mathrm{F}^{2}$

Final R indices [I $>2 \operatorname{sigma}(\mathrm{I})]$

$\mathrm{R}$ indices (all data)

Extinction coefficient

Largest diff. peak and hole 3b

$\mathrm{C}_{18} \mathrm{H}_{15} \mathrm{NO}_{4}$

309.31

273(2) K

$0.71073 \AA$

Triclinic

P-1

$\mathrm{a}=8.241(8) \AA$

$\alpha=78.183(14)^{\circ}$.

$\mathrm{b}=9.111(8) \AA$

$\beta=74.755(13)^{\circ}$.

$\mathrm{c}=10.887(10) \AA$

$\gamma=69.238(14)^{\circ}$.

$731.8(12) \AA^{3}$

2

$1.404 \mathrm{Mg} / \mathrm{m}^{3}$

$0.100 \mathrm{~mm}^{-1}$

330

$0.4 \times 0.35 \times 0.2 \mathrm{~mm}^{3}$

2.409 to $27.366^{\circ}$.

$-10<=\mathrm{h}<=10,-11<=\mathrm{k}<=10,-13<=\mathrm{l}<=14$

6316

$3324[\mathrm{R}($ int $)=0.0451]$

$97.9 \%$

None

Full-matrix least-squares on $\mathrm{F}^{2}$

3192 / 0 / 209

1.093

$\mathrm{R} 1=0.0635, \mathrm{wR} 2=0.1702$

$\mathrm{R} 1=0.1329, \mathrm{wR} 2=0.2230$

$\mathrm{n} / \mathrm{a}$

0.369 and -0.394 e. $\AA^{-3}$ 
Table S2. Crystal data and structure refinement for $4 \mathrm{~b}$

Identification code

Empirical formula

Formula weight

Temperature

Wavelength

Crystal system

Space group

Unit cell dimensions

Volume

Z

Density (calculated)

Absorption coefficient

$\mathrm{F}(000)$

Crystal size

Theta range for data collection

Index ranges

Reflections collected

Independent reflections

Completeness to theta $=25.242^{\circ}$

Absorption correction

Refinement method

Data / restraints / parameters

Goodness-of-fit on $\mathrm{F}^{2}$

Final R indices [I $>2 \operatorname{sigma}(\mathrm{I})]$

$\mathrm{R}$ indices (all data)

Extinction coefficient

Largest diff. peak and hole 4b

$\mathrm{C}_{21} \mathrm{H}_{19} \mathrm{NO}_{3}$

333.37

296(2) K

$0.71073 \AA$

Monoclinic

$\mathrm{P} 21 / \mathrm{c}$

$\mathrm{a}=13.371(12) \AA$

$\alpha=90^{\circ}$.

$\mathrm{b}=17.608(15) \AA$

$\beta=102.646(13)^{\circ}$.

$c=7.456(6) \AA$

$\gamma=90^{\circ}$.
4

$1.293 \mathrm{Mg} / \mathrm{m}^{3}$

$0.087 \mathrm{~mm}^{-1}$

704

$0.35 \times 0.33 \times 0.25 \mathrm{~mm}^{3}$

1.561 to $26.710^{\circ}$.

$-16<=\mathrm{h}<=16,-18<=\mathrm{k}<=22,-9<=1<=9$

14429

$3632[\mathrm{R}($ int $)=0.0627]$

$99.6 \%$

None

Full-matrix least-squares on $\mathrm{F}^{2}$

3579 / 0 / 227

1.049

$\mathrm{R} 1=0.0629, \mathrm{wR} 2=0.2032$

$\mathrm{R} 1=0.0869, \mathrm{wR} 2=0.2430$

$\mathrm{n} / \mathrm{a}$

0.420 and -0.389 e. $\AA^{-3}$ 


\section{References}

1. Qi, Z.; Wang, S. Copper-Catalyzed $\beta$-Lactam Formation Initiated by 1,3-Azaprotio Transfer of Oximes and Methyl Propiolate. Org. Lett. 2019, 23, 5777-5781.

2. Chan, C.-M.; Xing, Q.; Chow, Y.-C.; Hung, S.-F.; Yu, W.-Y. Photoredox Decarboxylative $\mathrm{C}\left(\mathrm{sp}^{3}\right)$-N Coupling of $\alpha$-Diazoacetates with Alkyl $N$-Hydroxyphthalimide Esters for Diversified Synthesis of Functionalized N-Alkyl Hydrazones. Org. Lett. 2019, 21, 8037-8043.

3. Guptill, D. M.; Cohen, C. M.; Davies, H. M. L. Rhodium(II)-Catalyzed Stereoselective Synthesis of Allylsilanes. Org. Lett. 2013, 15, 6120-6123.

4. Hari, D. P.; Waser, J. Copper-Catalyzed Oxy-Alkynylation of Diazo Compounds with Hypervalent Iodine Reagents. J. Am. Chem. Soc. 2016, 138, 2190-2193.

5. Zhang, Z.; He, Z.; Xie, Y.; He, T.; Fu, Y.; Yu, Y.; Huang, F. Brønsted Acid-Catalyzed Homogeneous O-H and S-H Insertion Reactions under Metal- and Ligandfree Conditions. Org. Chem. Front. 2021, 8, 1233-1242.

6. Sheldrick, G. M. Crystal Structure Refinement with SHELXL. Acta Cryst. Acta Cryst. 2015, A71, 3-8.

7. Sheldrick, G. M. Crystal Structure Refinement with SHELXL. Acta Cryst. 2015, C71, 3-8. 


\section{Copies of NMR spectra}

${ }^{1} \mathrm{H}$ NMR of 3a $\left(400 \mathrm{MHz}, \mathrm{CDCl}_{3}\right)$
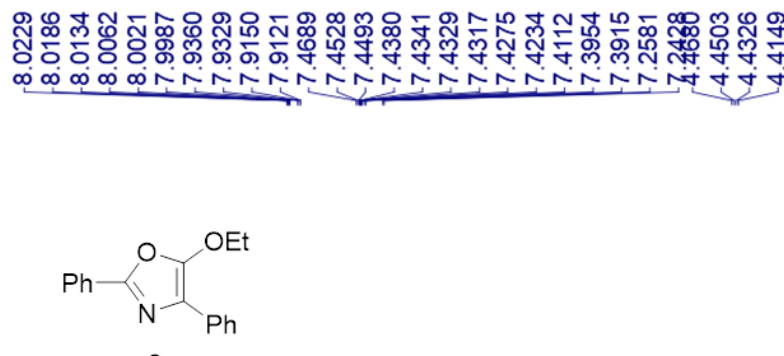

3a

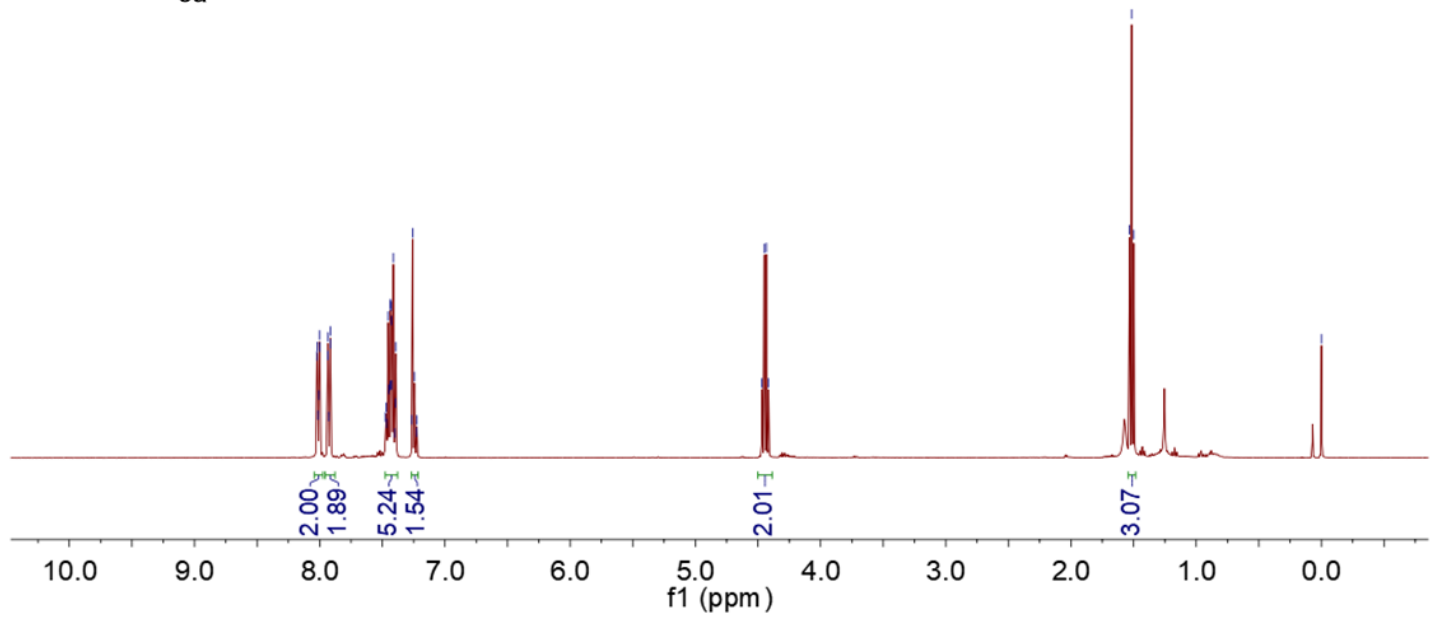

${ }^{13} \mathrm{C}$ NMR of 3a $\left(100 \mathrm{MHz}, \mathrm{CDCl}_{3}\right)$
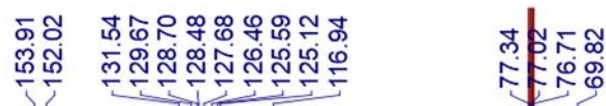

ํㅗำ

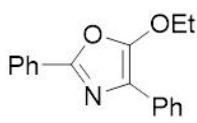

3a

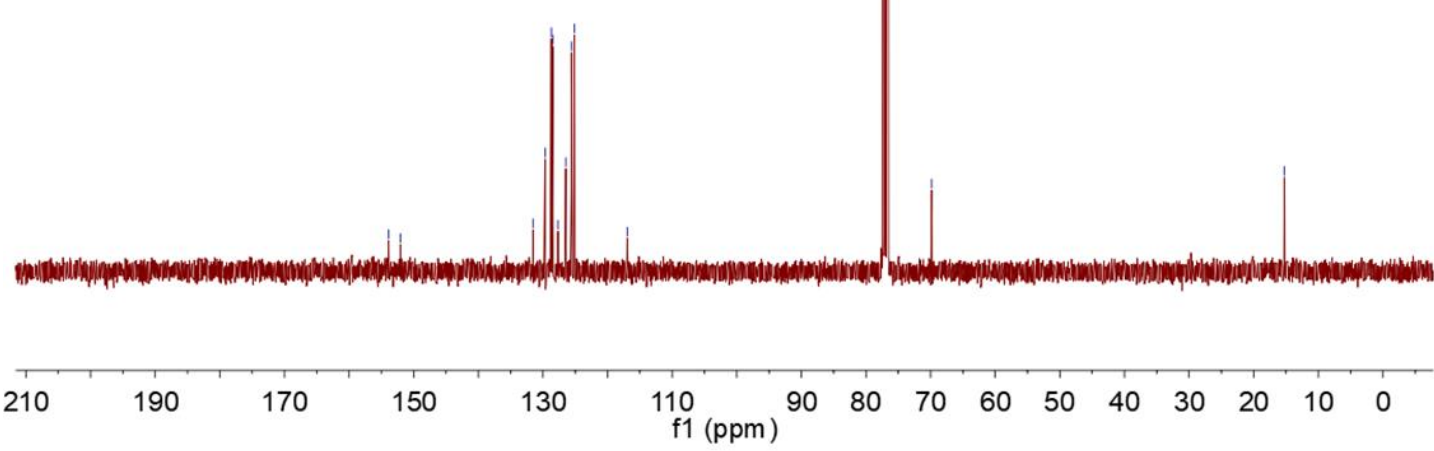


${ }^{1} \mathrm{H}$ NMR of $\mathbf{3 b}\left(400 \mathrm{MHz}, \mathrm{CDCl}_{3}\right)$

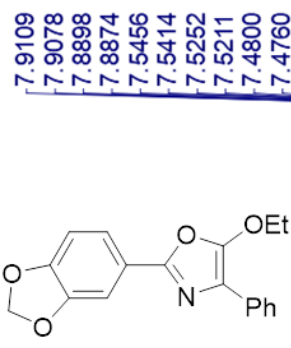

$3 b$

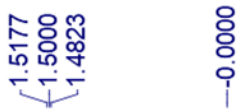

产高范宮

ن千+子

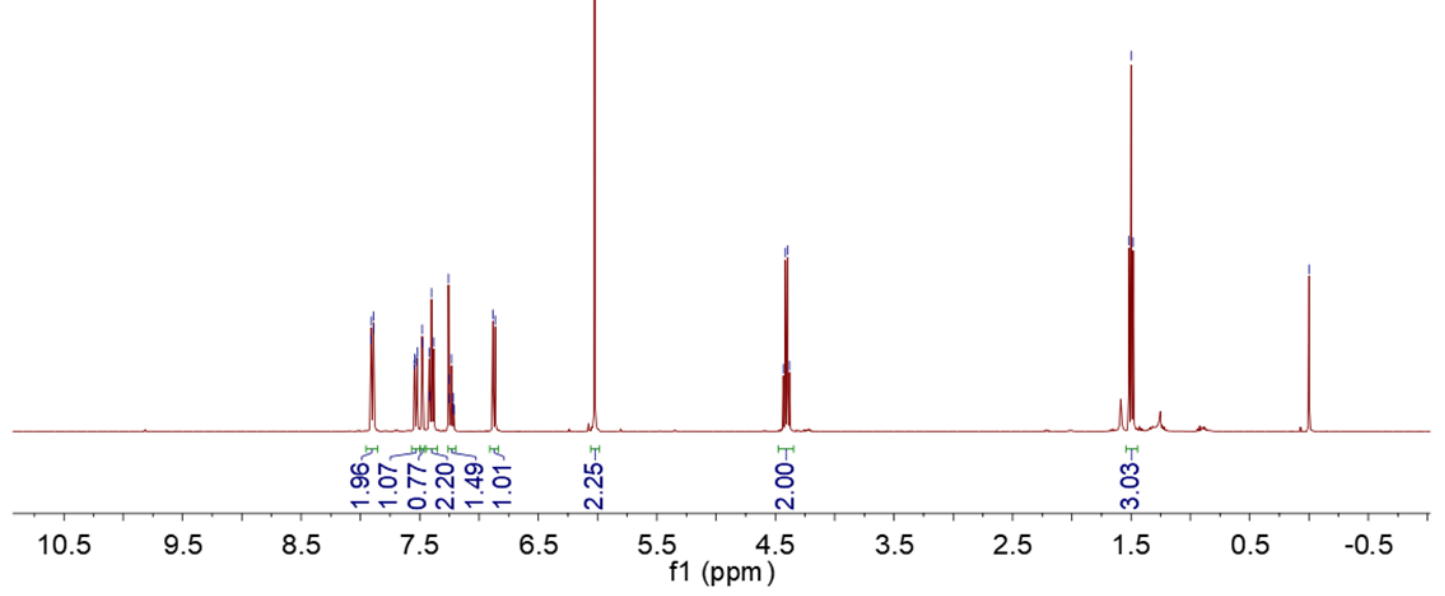

${ }^{13} \mathrm{C}$ NMR of $\mathbf{3 b}\left(100 \mathrm{MHz}, \mathrm{CDCl}_{3}\right)$

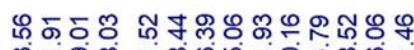

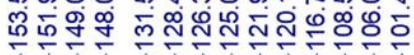

స్లిం\%ळ

ヘペツ

กิ

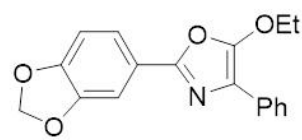

$3 b$

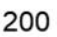

180

160

140

120

100
f1 $(p p m)$

80

60

40

20 
${ }^{1} \mathrm{H}$ NMR of $3 \mathbf{c}\left(400 \mathrm{MHz}\right.$, DMSO- $\left.d_{6}\right)$

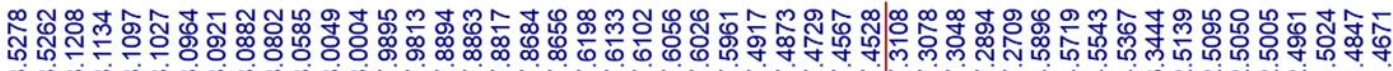

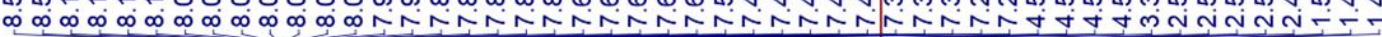

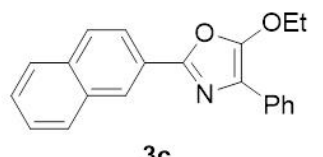

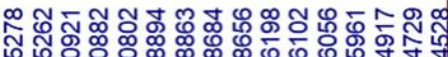

$\infty \infty \infty \infty \infty N N N$ NNNNNN

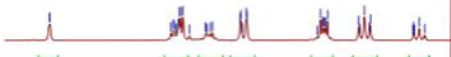

3c

\begin{tabular}{|c|c|c|}
\hline & & \\
\hline 8 & ô के & ్లో \\
\hline 8.5 & $\begin{array}{c}8.0 \\
\text { f1 }(\mathrm{ppm}\end{array}$ & 7.5 \\
\hline
\end{tabular}

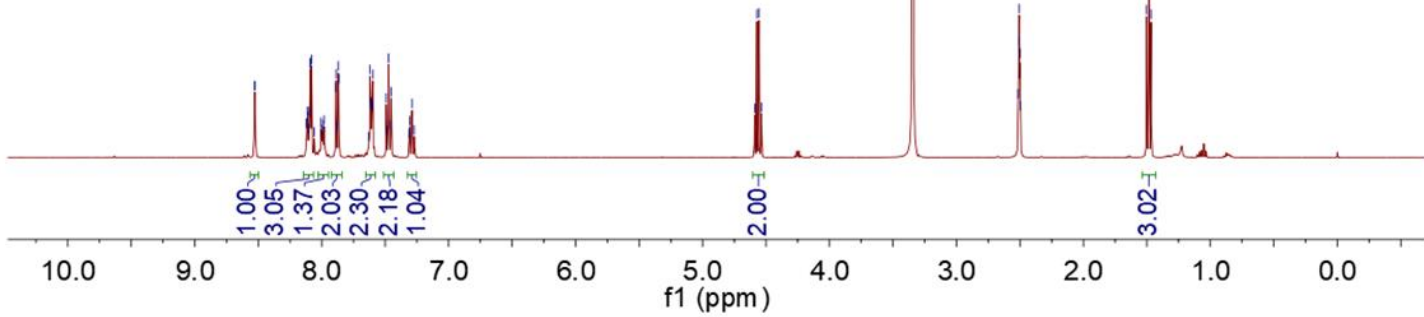

${ }^{13} \mathrm{C}$ NMR of $3 \mathbf{c}\left(100 \mathrm{MHz}, \mathrm{DMSO}-d_{6}\right)$

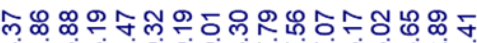

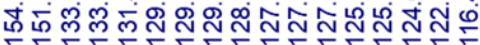
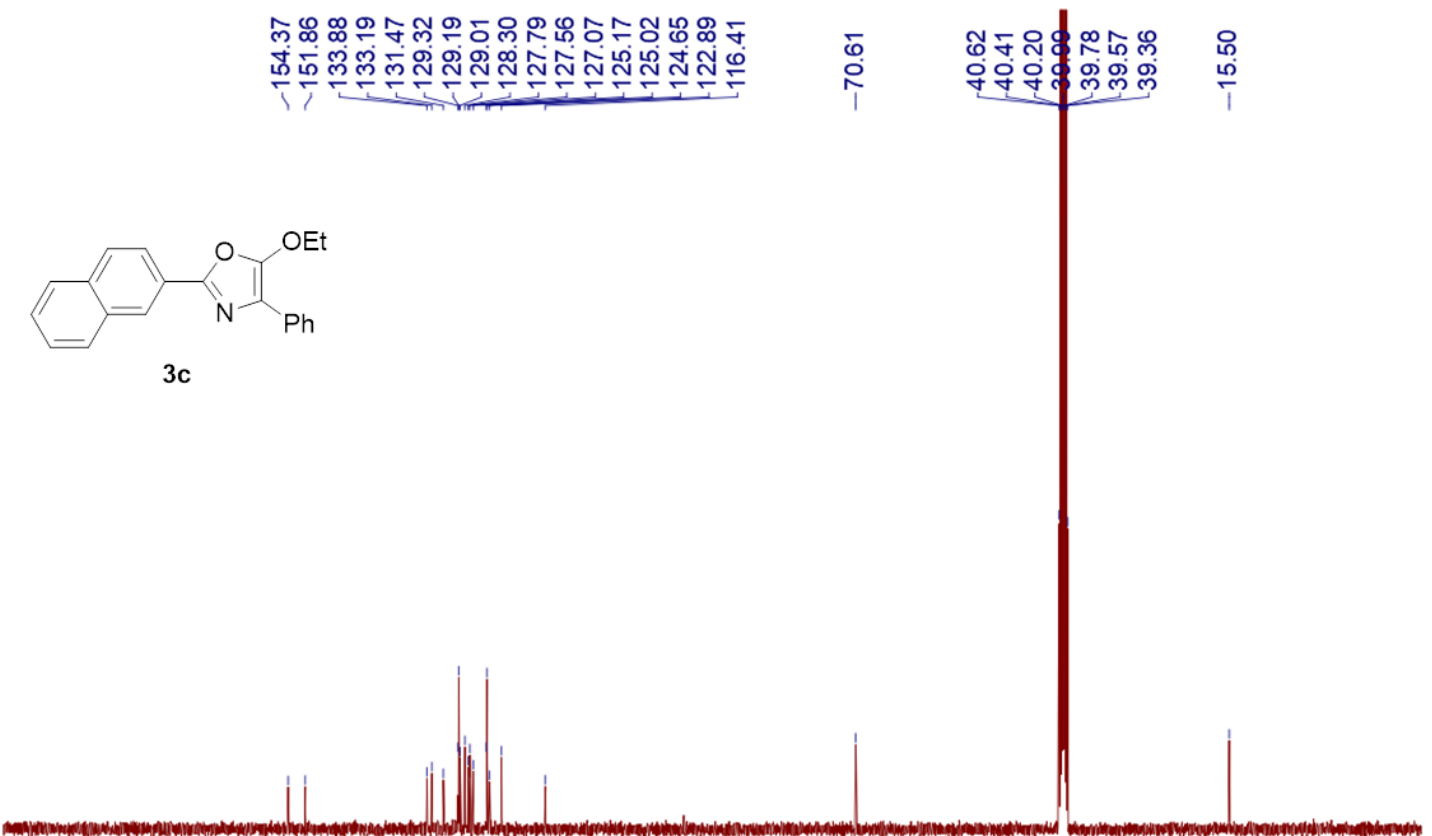

190

170

150

130

110

90

f1 (ppm)

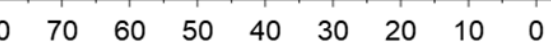


${ }^{1} \mathrm{H}$ NMR of 3d (400 MHz, DMSO- $\left.d_{6}\right)$

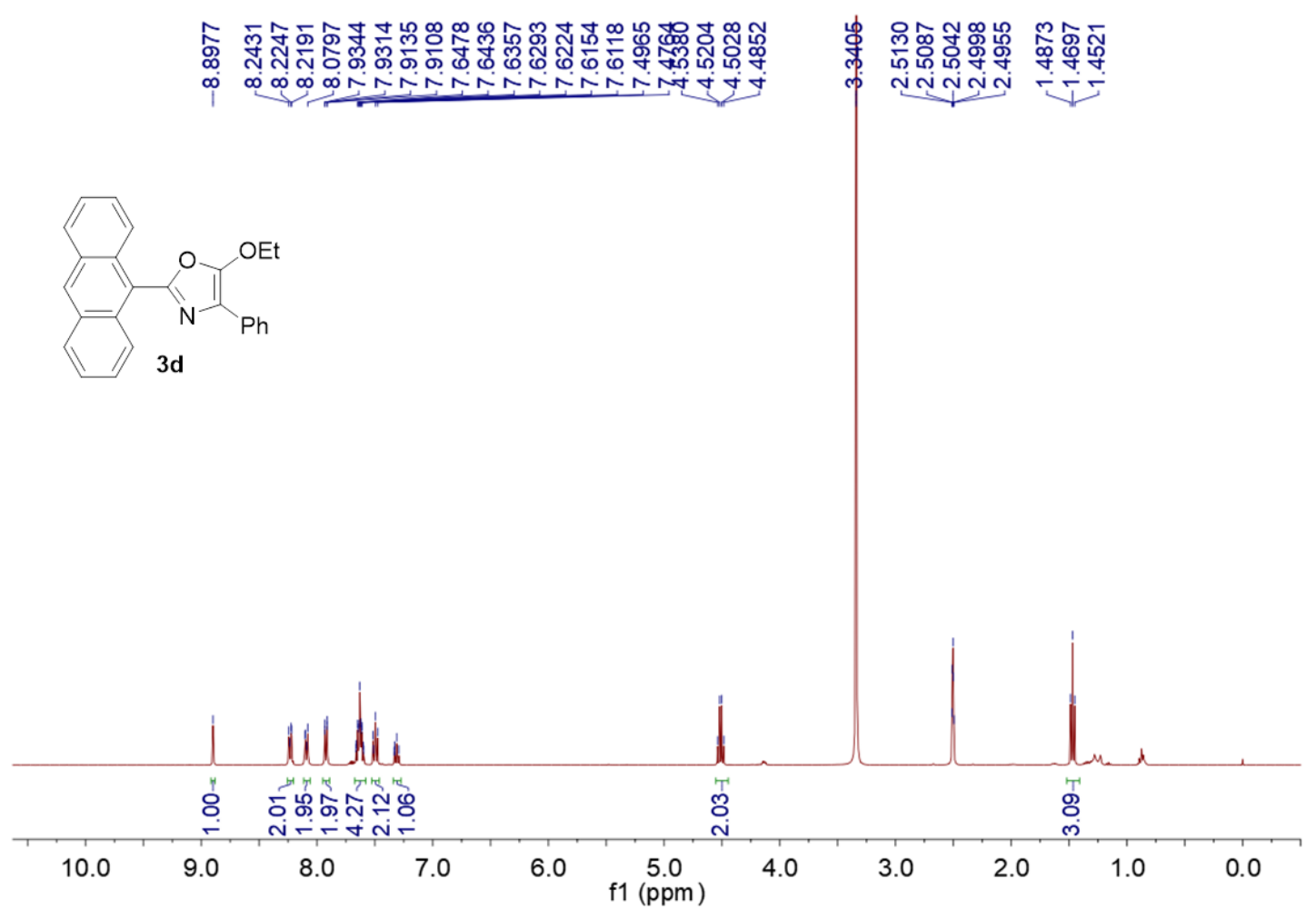

${ }^{13} \mathrm{C}$ NMR of $3 d\left(100 \mathrm{MHz}\right.$, DMSO- $\left.d_{6}\right)$

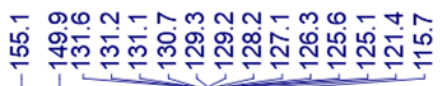

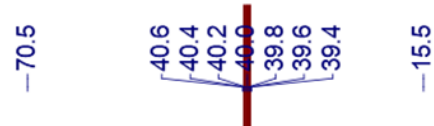
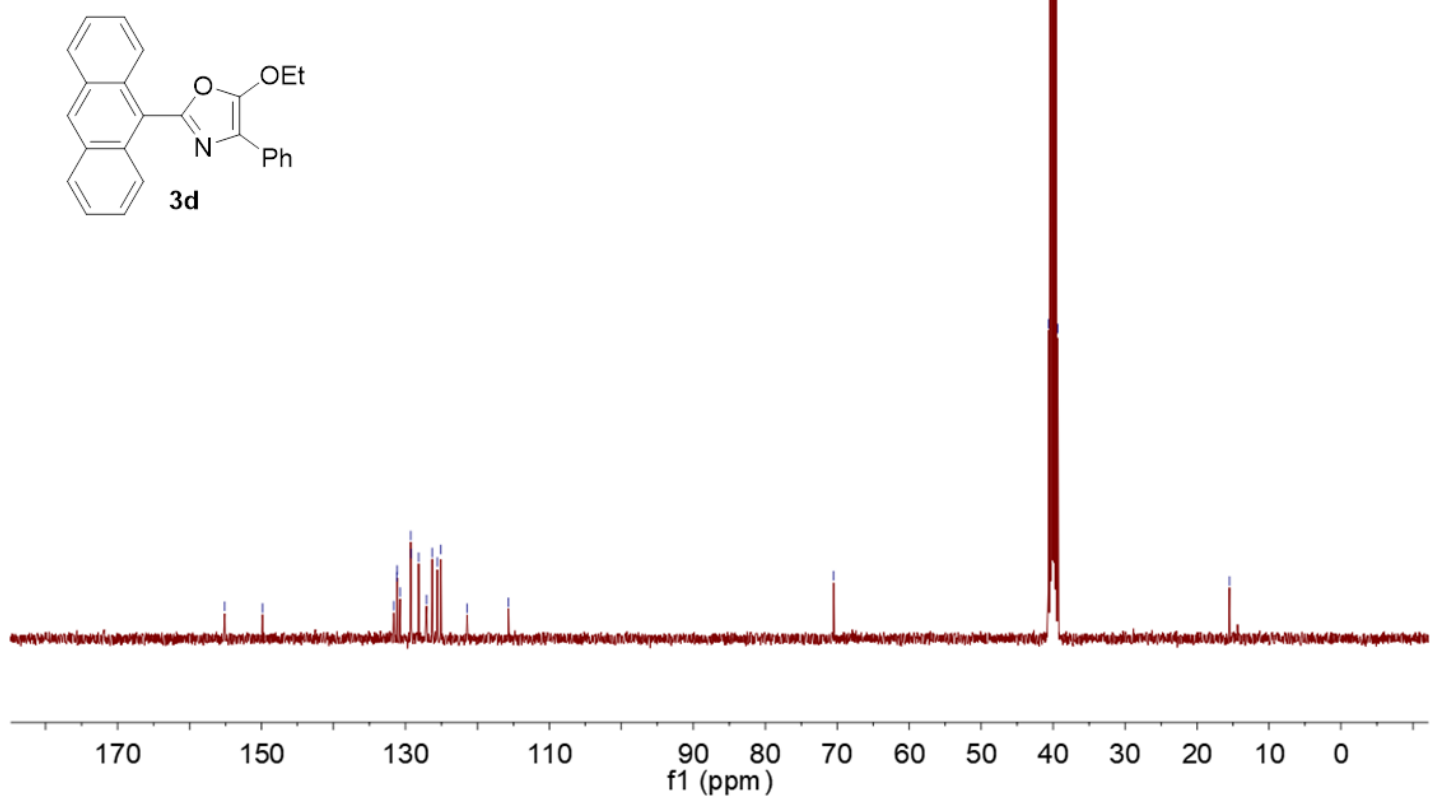
${ }^{1} \mathrm{H}$ NMR of $3 \mathbf{e}\left(400 \mathrm{MHz}, \mathrm{CDCl}_{3}\right)$

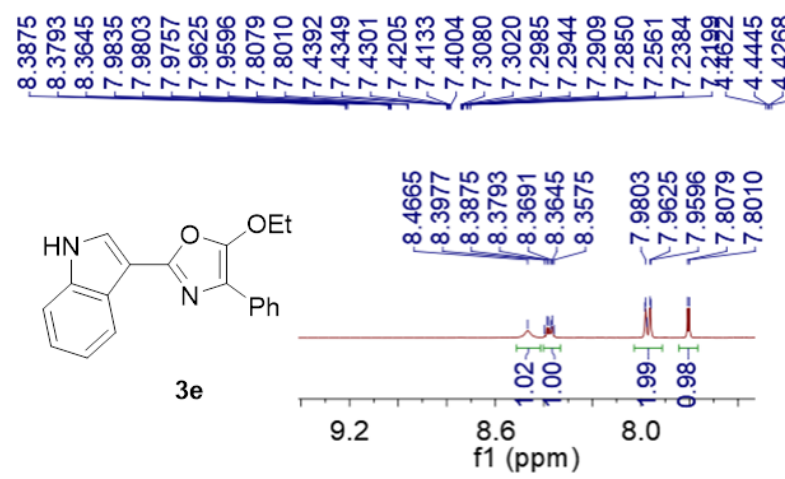

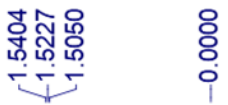

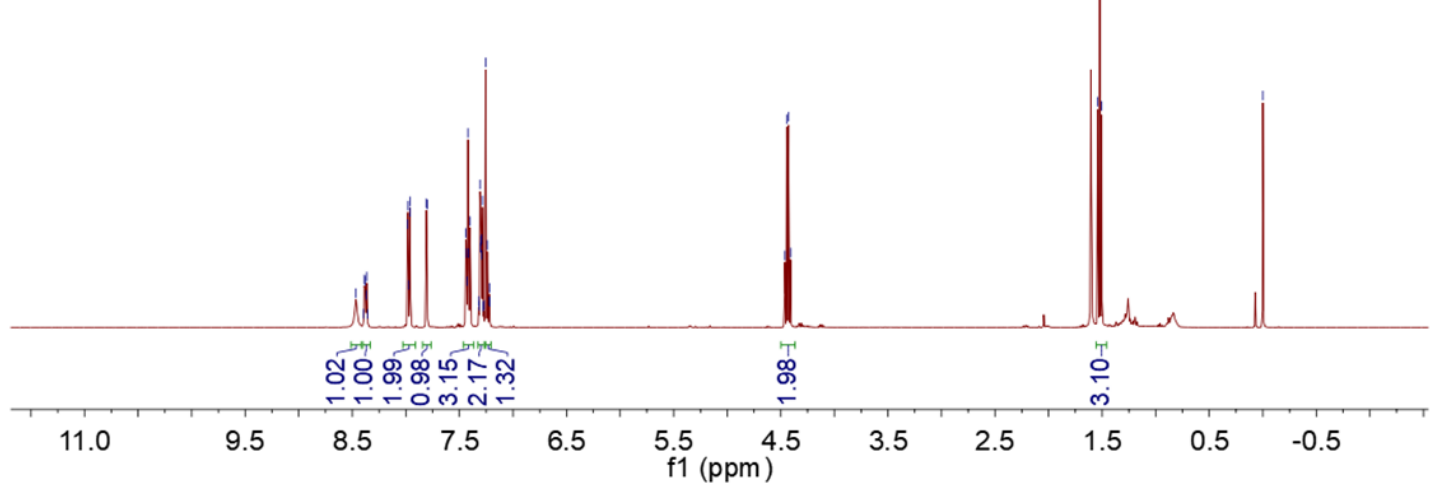

${ }^{13} \mathrm{C}$ NMR of $3 \mathbf{e}\left(100 \mathrm{MHz}, \mathrm{CDCl}_{3}\right)$

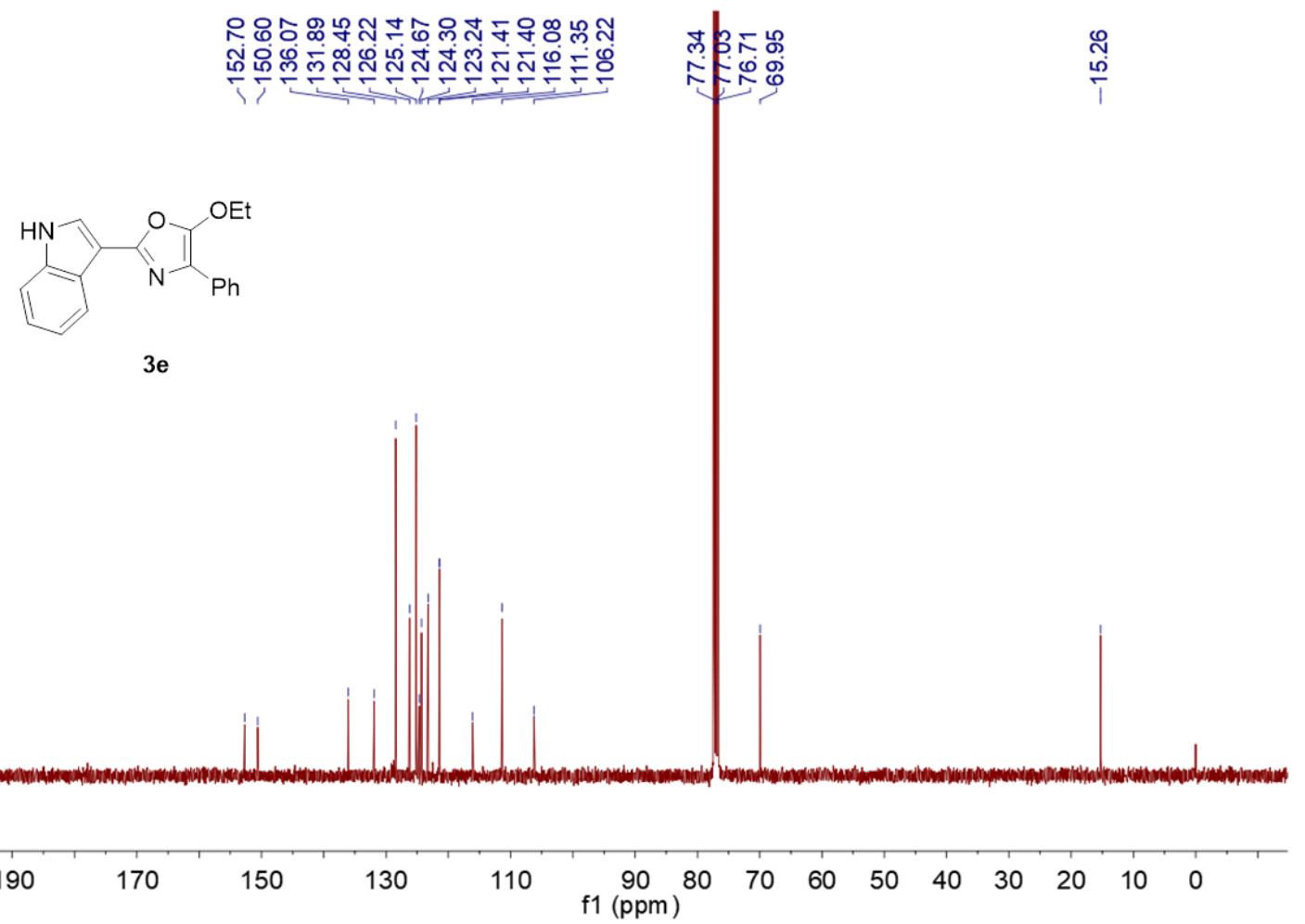


${ }^{1} \mathrm{H}$ NMR of $\mathbf{3 f}\left(400 \mathrm{MHz}, \mathrm{CDCl}_{3}\right)$
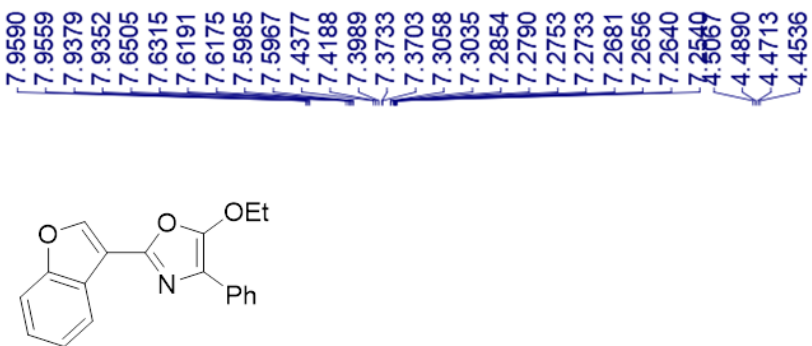

$3 f$

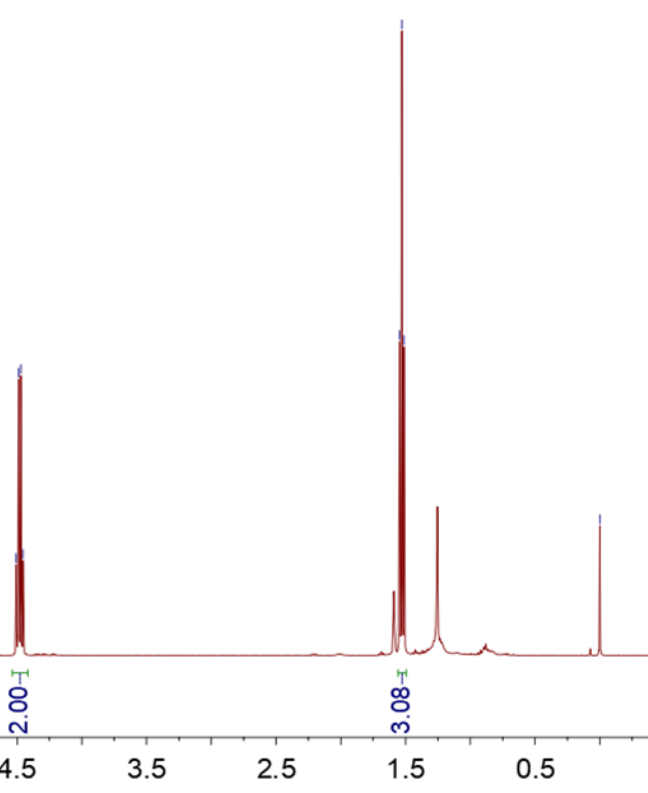

10.5
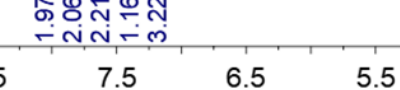

f1 (ppm)

${ }^{13} \mathrm{C}$ NMR of $3 \mathbf{f}\left(100 \mathrm{MHz}, \mathrm{CDCl}_{3}\right)$

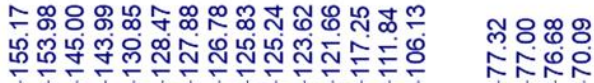

$\frac{N}{1}$

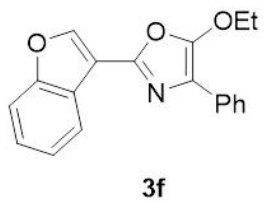

$3 f$

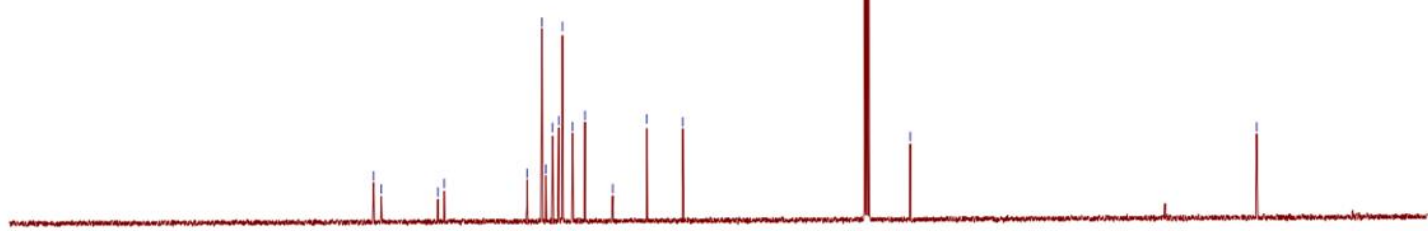

210

190

$170 \quad 150$

130

90 
${ }^{1} \mathrm{H}$ NMR of $\mathbf{3 g}\left(400 \mathrm{MHz}, \mathrm{CDCl}_{3}\right)$
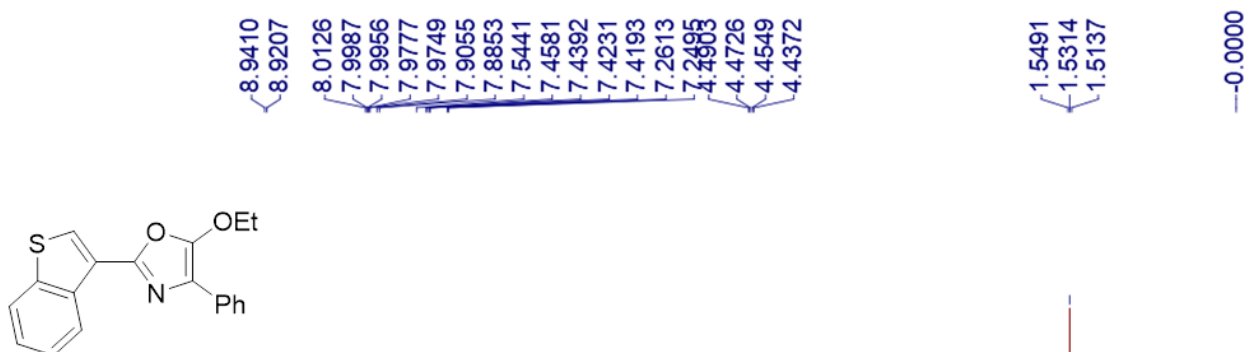

$3 g$

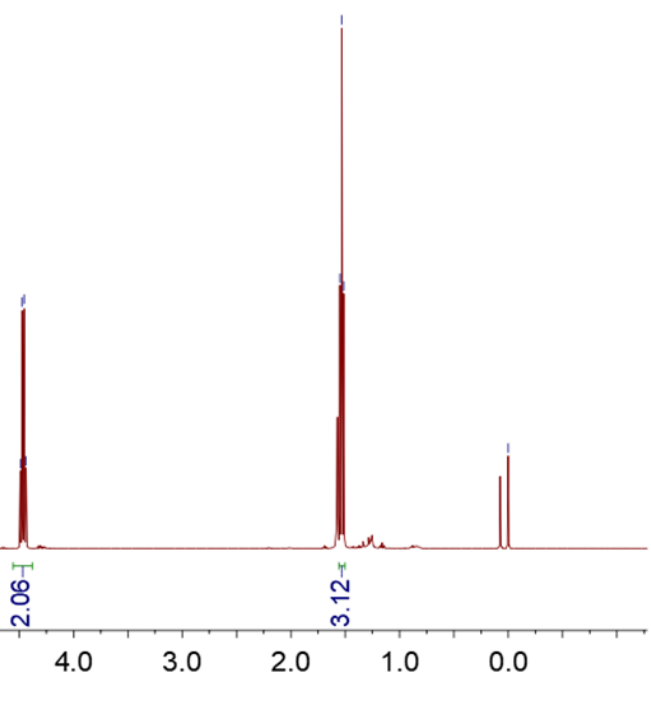

${ }^{13} \mathrm{C}$ NMR of $\mathbf{3 g}\left(100 \mathrm{MHz}, \mathrm{CDCl}_{3}\right)$

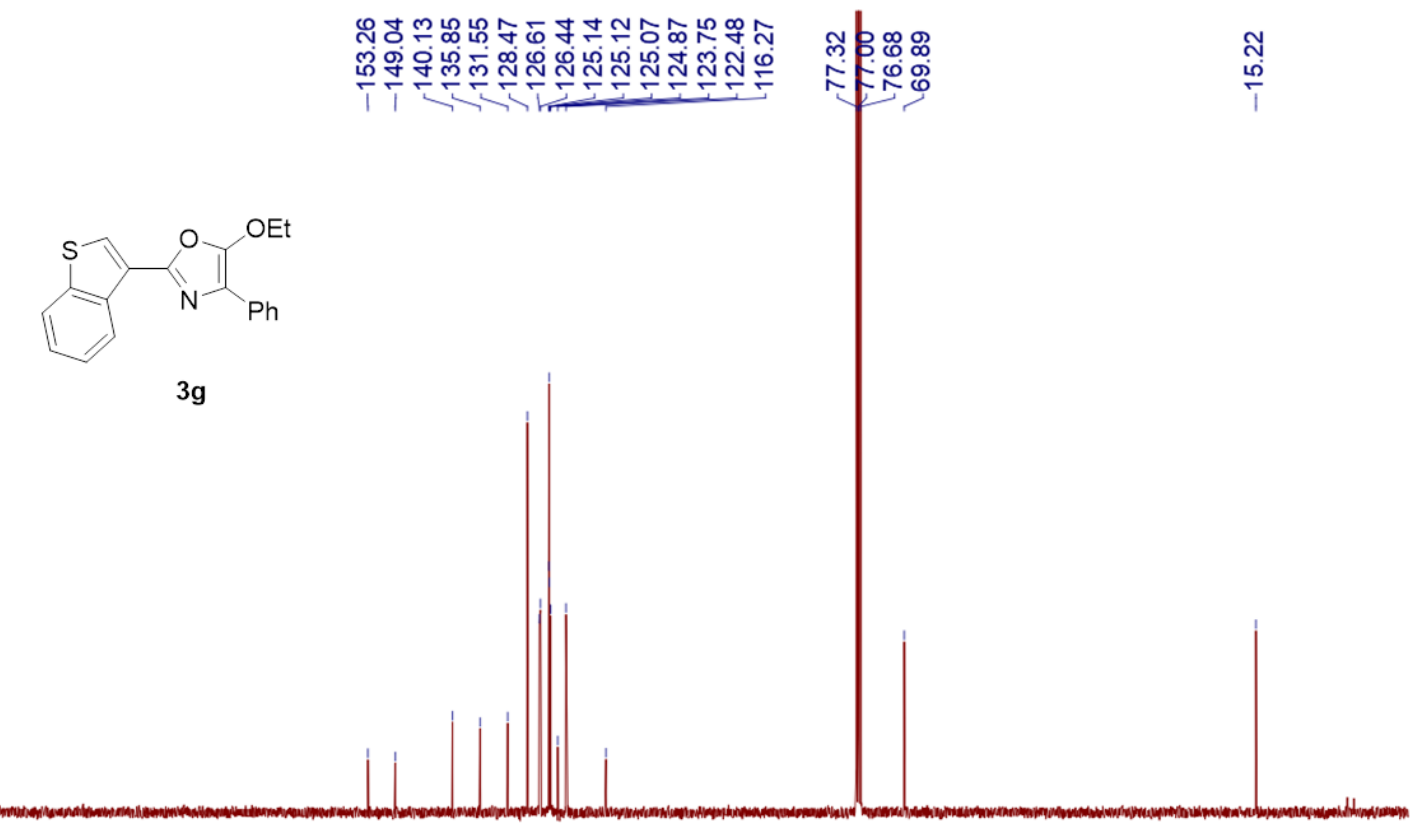

210

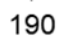

170

150

130

$90 \quad 80 \quad 70 \quad 60$

f1 (ppm) 
${ }^{1} \mathrm{H}$ NMR of $\mathbf{3 h}\left(400 \mathrm{MHz}, \mathrm{CDCl}_{3}\right)$
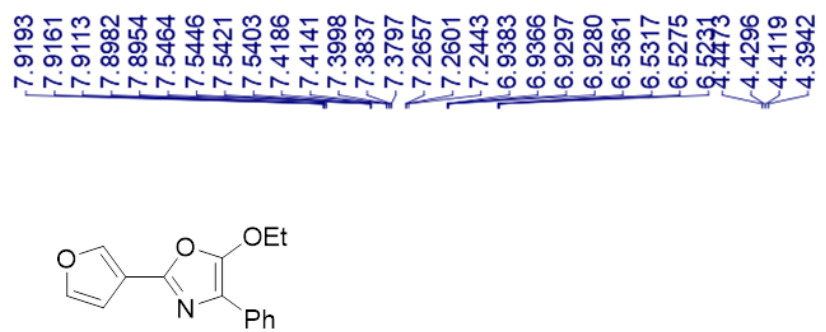

$3 \mathrm{~h}$

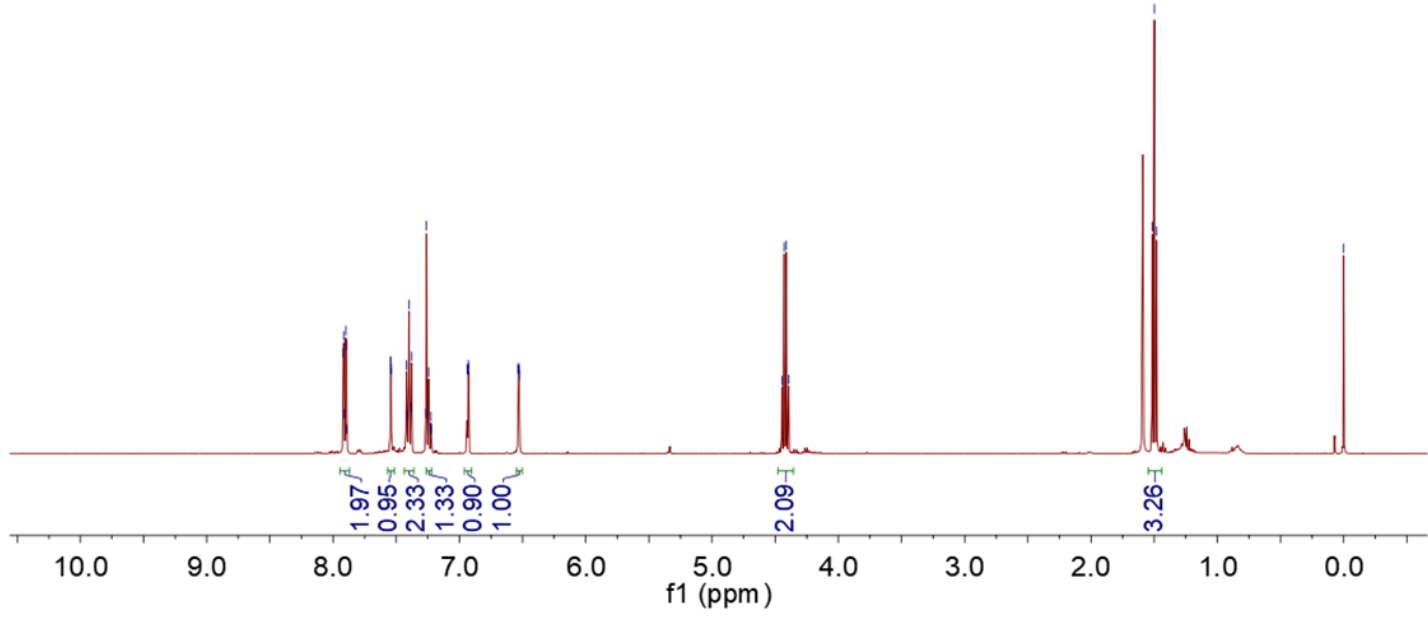

${ }^{13} \mathrm{C}$ NMR of $\mathbf{3 h}\left(100 \mathrm{MHz}, \mathrm{CDCl}_{3}\right)$

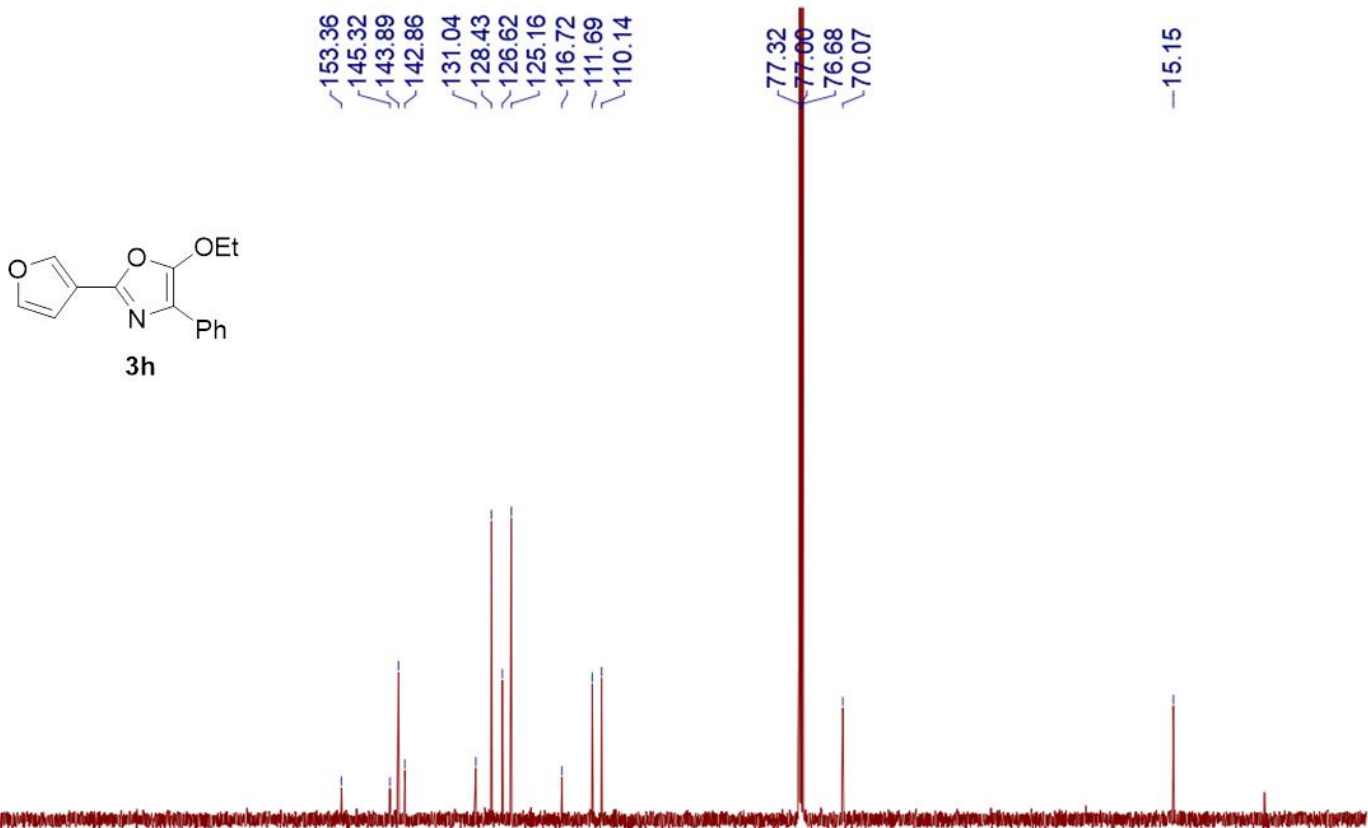


${ }^{1} \mathrm{H}$ NMR of $3 \mathbf{i}\left(400 \mathrm{MHz}, \mathrm{CDCl}_{3}\right)$

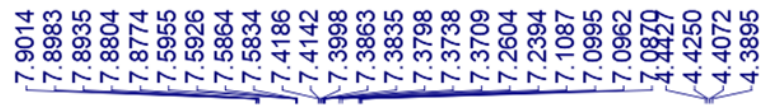

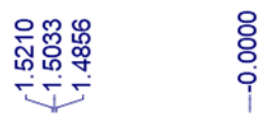

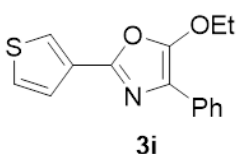

$3 i$

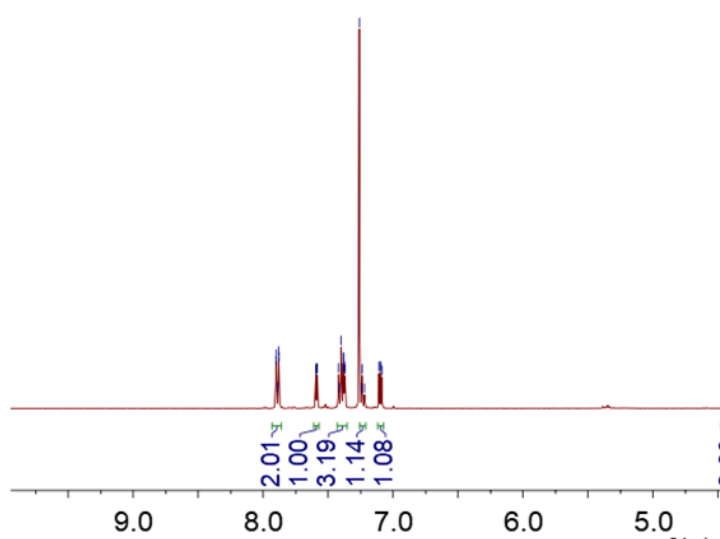

f1 (ppm)

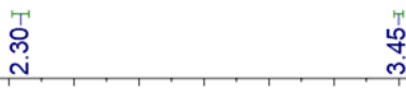

ֻे

${ }^{13} \mathrm{C}$ NMR of $3 \mathbf{i}\left(100 \mathrm{MHz}, \mathrm{CDCl}_{3}\right)$

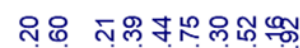

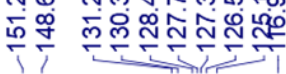

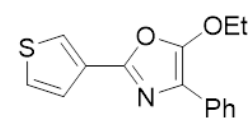

$3 i$

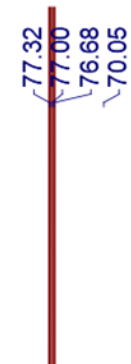

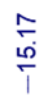

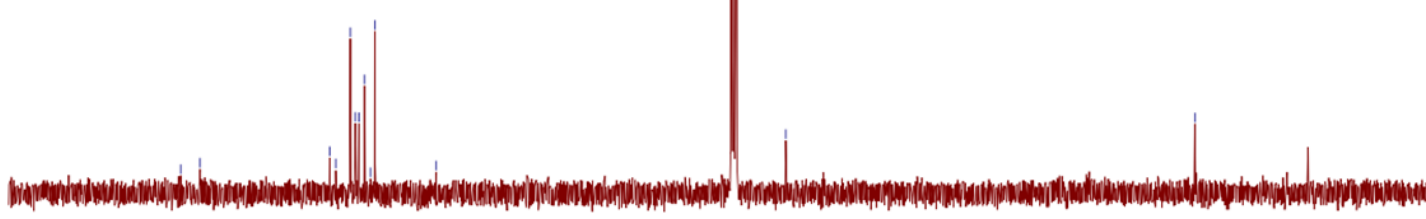

170

150

130

110

$90 \underset{\mathrm{f} 1(\mathrm{ppm})}{80} 70$

60

$50 \quad 40$

3020

$10 \quad 0 \quad-10$ 
${ }^{1} \mathrm{H}$ NMR of $\mathbf{3 j}\left(400 \mathrm{MHz}, \mathrm{CDCl}_{3}\right)$

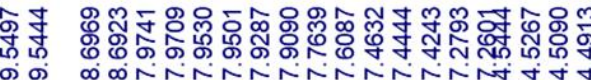

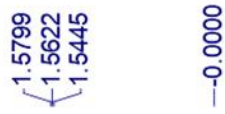

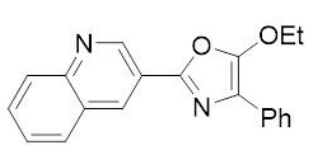

$3 \mathbf{j}$
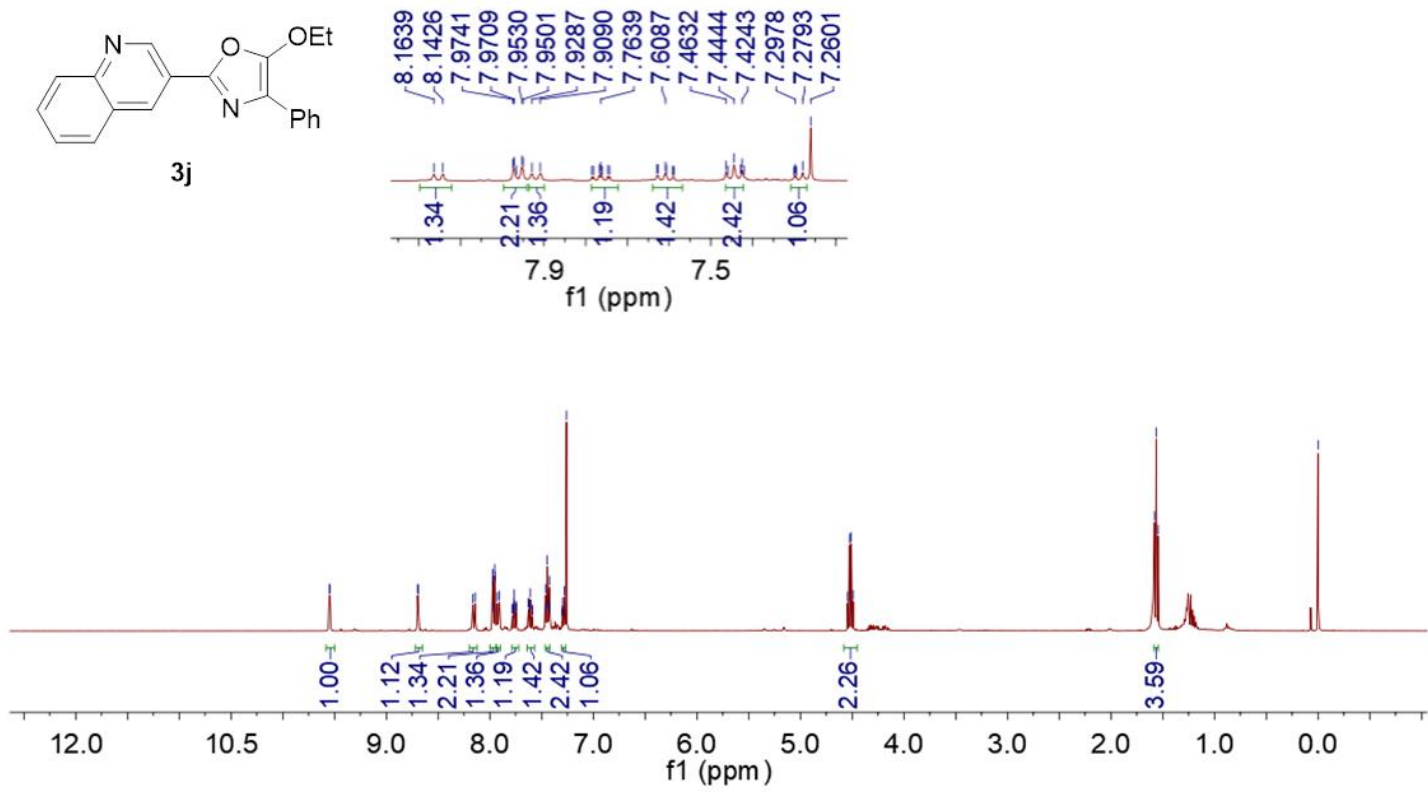

${ }^{13} \mathrm{C}$ NMR of $\mathbf{3 j}\left(100 \mathrm{MHz}, \mathrm{CDCl}_{3}\right)$
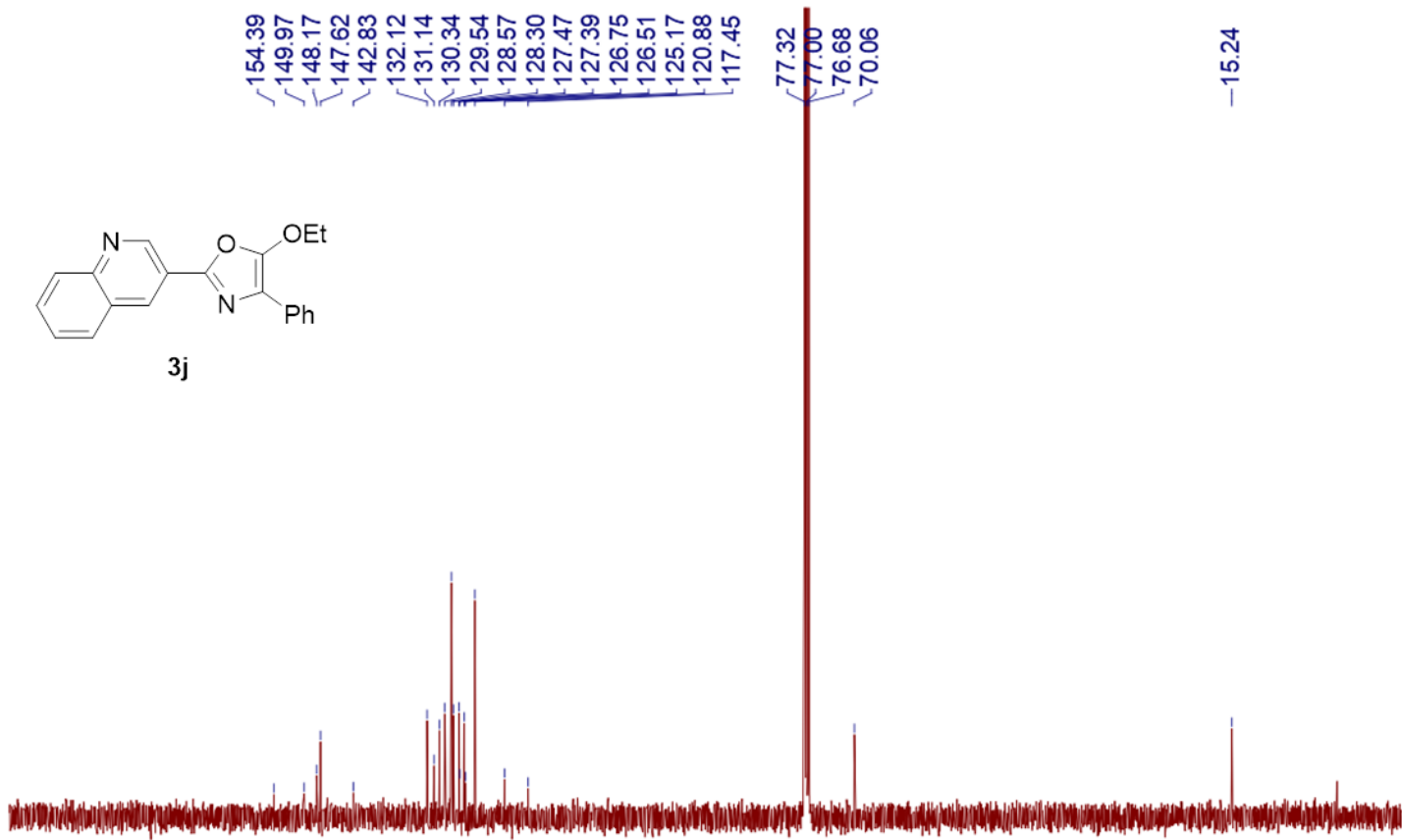

190

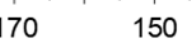

130

110

9080

$\begin{array}{lllllllll}70 & 60 & 50 & 40 & 30 & 20 & 10 & 0\end{array}$ 
${ }^{1} \mathrm{H} \mathrm{NMR}$ of 3k $\left(400 \mathrm{MHz}, \mathrm{CDCl}_{3}\right)$

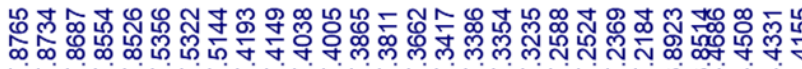

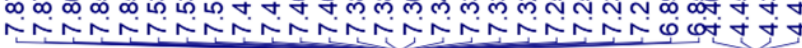

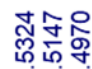

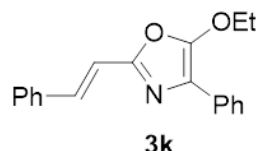

$3 k$

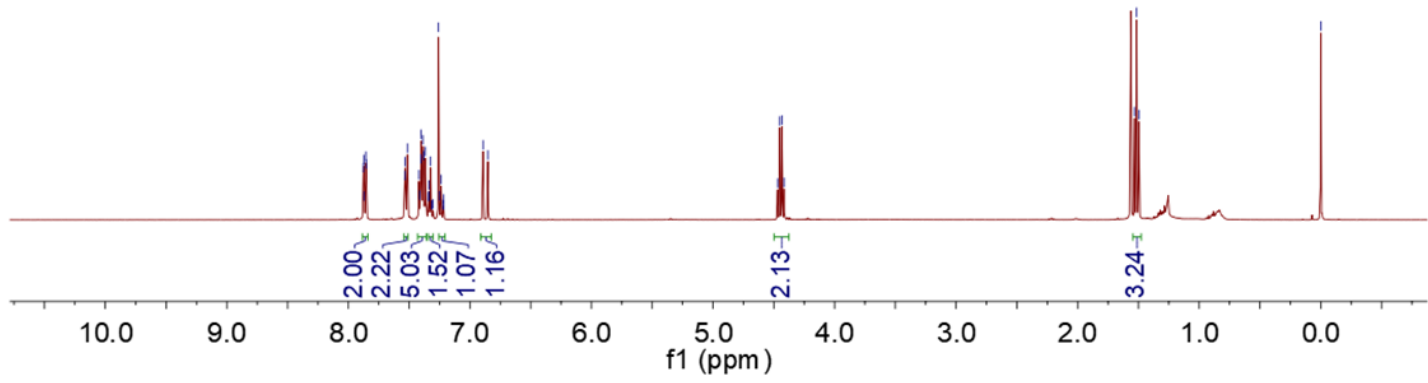

${ }^{13} \mathrm{C}$ NMR of 3k $\left(100 \mathrm{MHz}, \mathrm{CDCl}_{3}\right)$
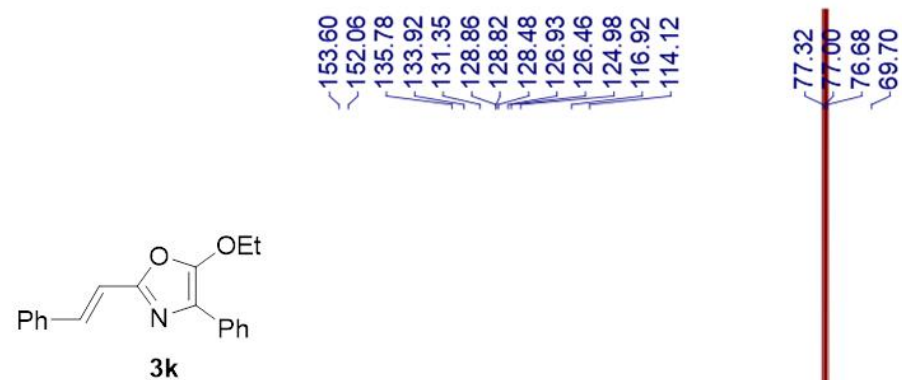

กิ

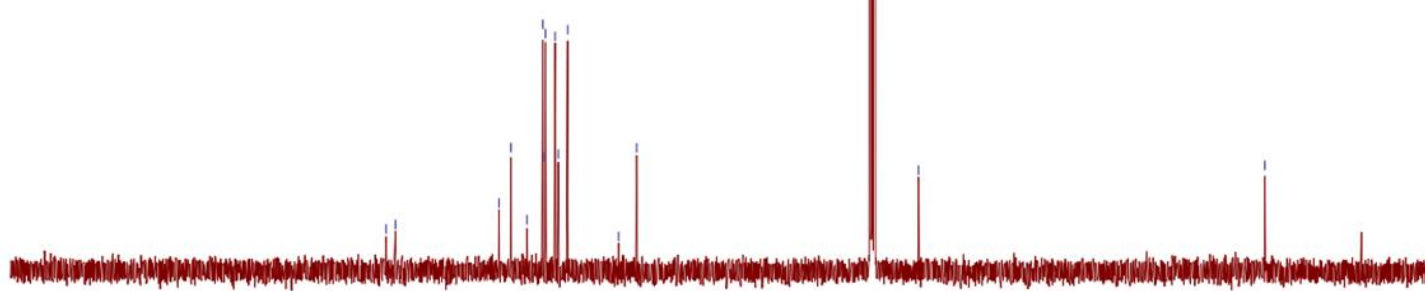

210 190 170 150 130 110 f1 (ppm) $\begin{array}{llllllllll}90 & 80 & 70 & 60 & 50 & 40 & 30 & 20 & 10 & 0\end{array}$ 
${ }^{1} \mathrm{H}$ NMR of $3 \mathbf{l}\left(400 \mathrm{MHz}, \mathrm{CDCl}_{3}\right)$
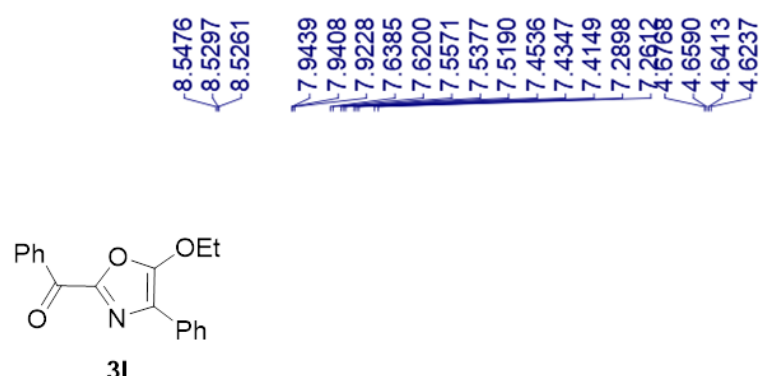

31

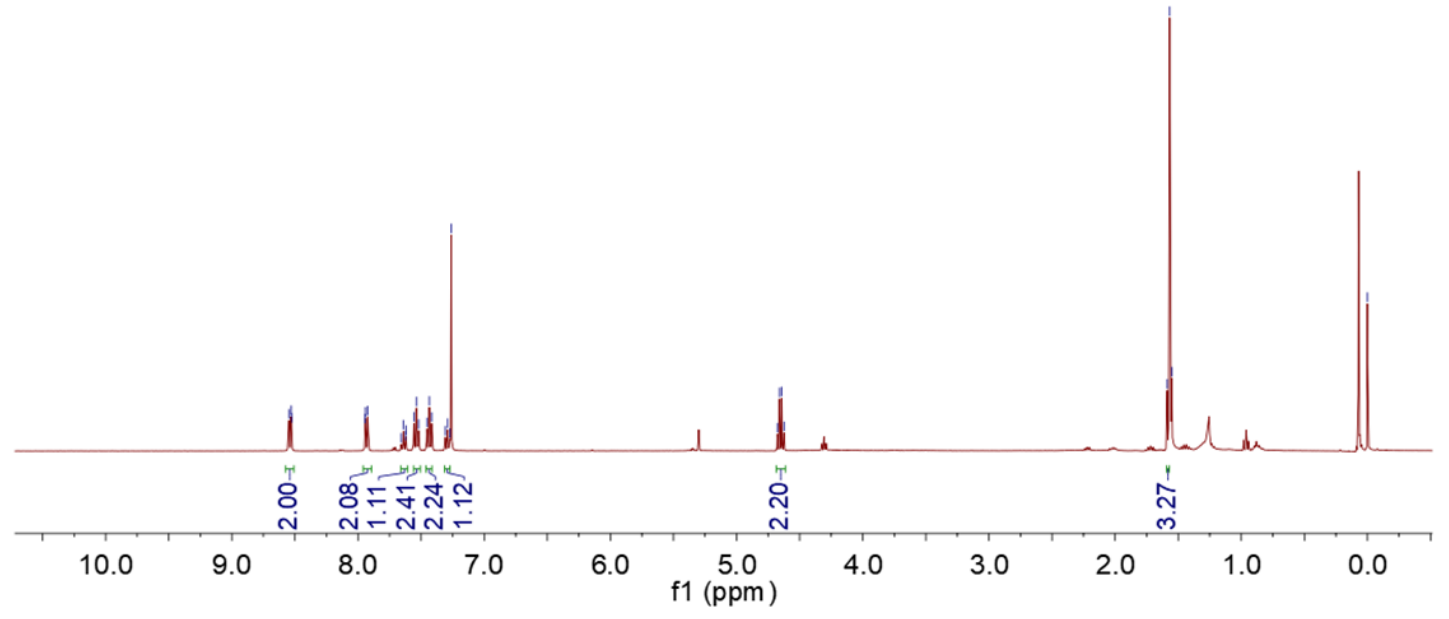

${ }^{13} \mathrm{C}$ NMR of $3 \mathrm{l}\left(100 \mathrm{MHz}, \mathrm{CDCl}_{3}\right)$

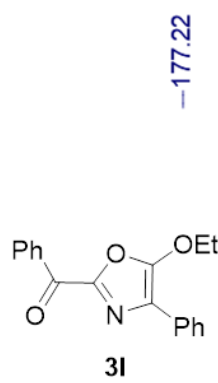

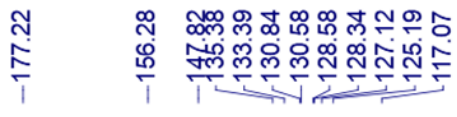

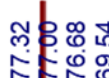

음

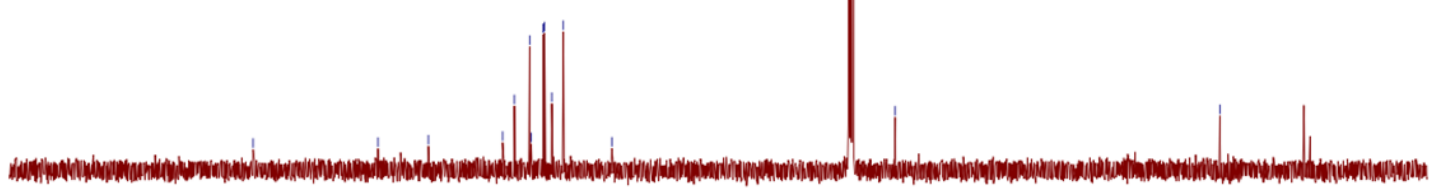


${ }^{1} \mathrm{H}$ NMR of $\mathbf{3 m}\left(400 \mathrm{MHz}, \mathrm{CDCl}_{3}\right)$

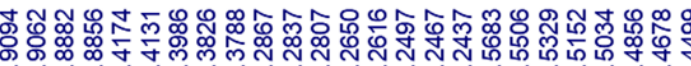

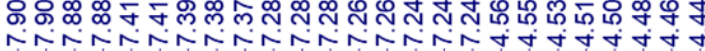

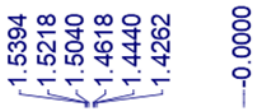

$\mathrm{EtO}_{2} \mathrm{C}-\mathrm{N}_{\mathrm{Ph}}^{\mathrm{OEt}}$

$3 m$

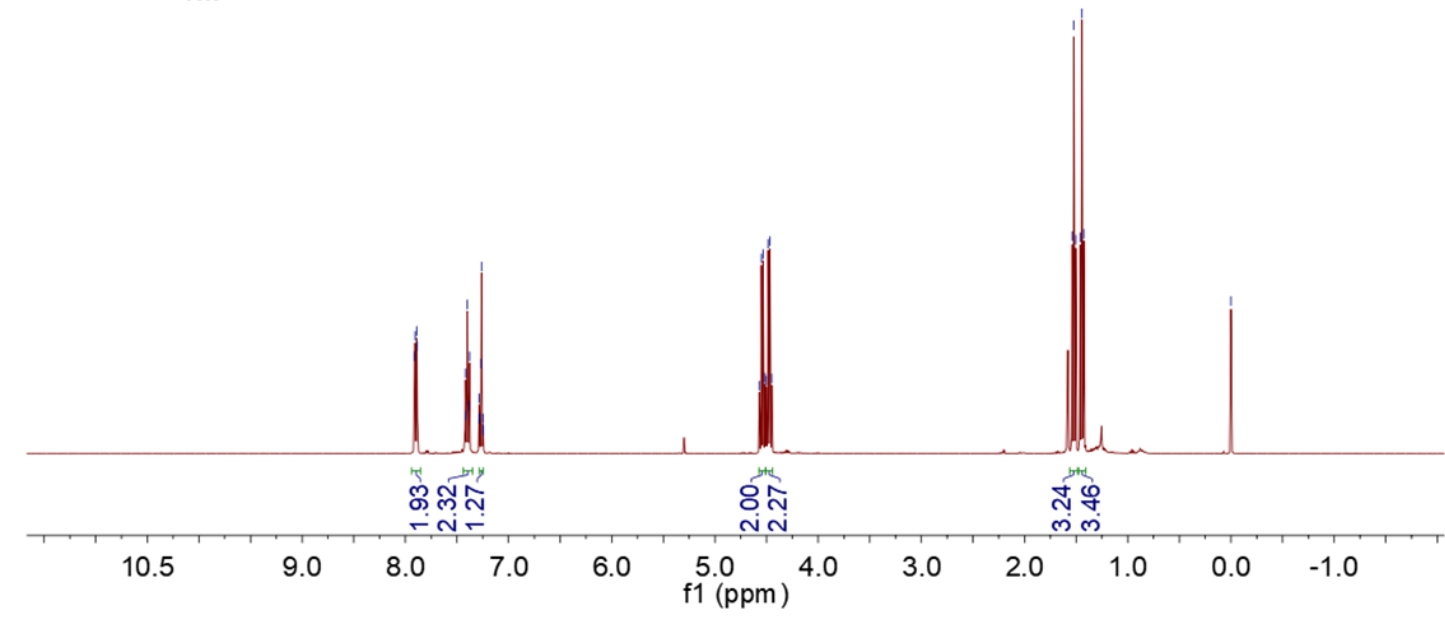

${ }^{13} \mathrm{C}$ NMR of $3 \mathbf{m}\left(100 \mathrm{MHz}, \mathrm{CDCl}_{3}\right)$

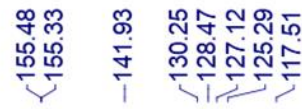

กิ:

$\varphi$ กิ

மீí

$\mathrm{EtO}_{2} \mathrm{C}$

$3 m$

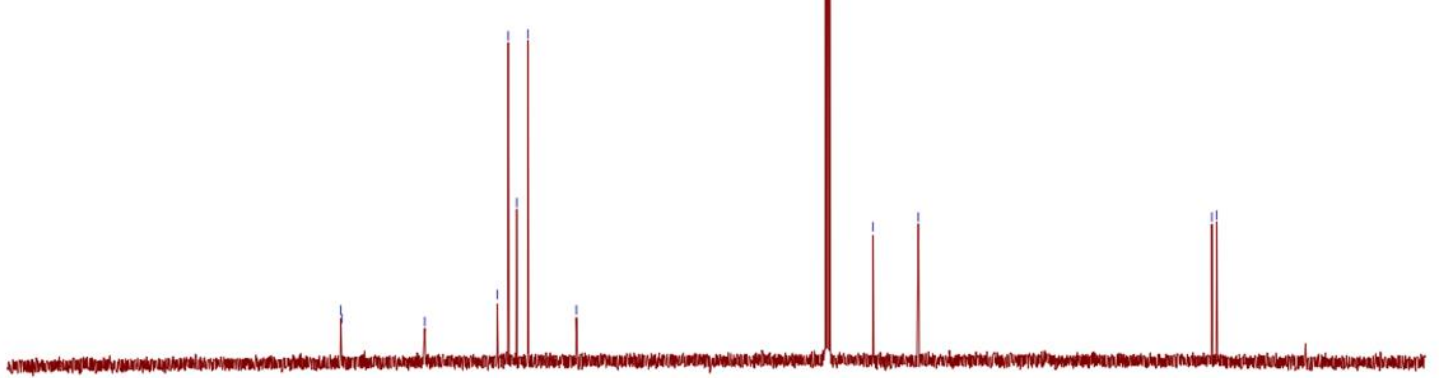

200

180

160

140

120

100

80

60

20

0

$1(\mathrm{ppm})$ 
${ }^{1} \mathrm{H}$ NMR of 3n $\left(400 \mathrm{MHz}, \mathrm{CDCl}_{3}\right)$

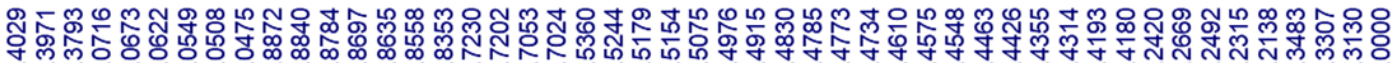

m
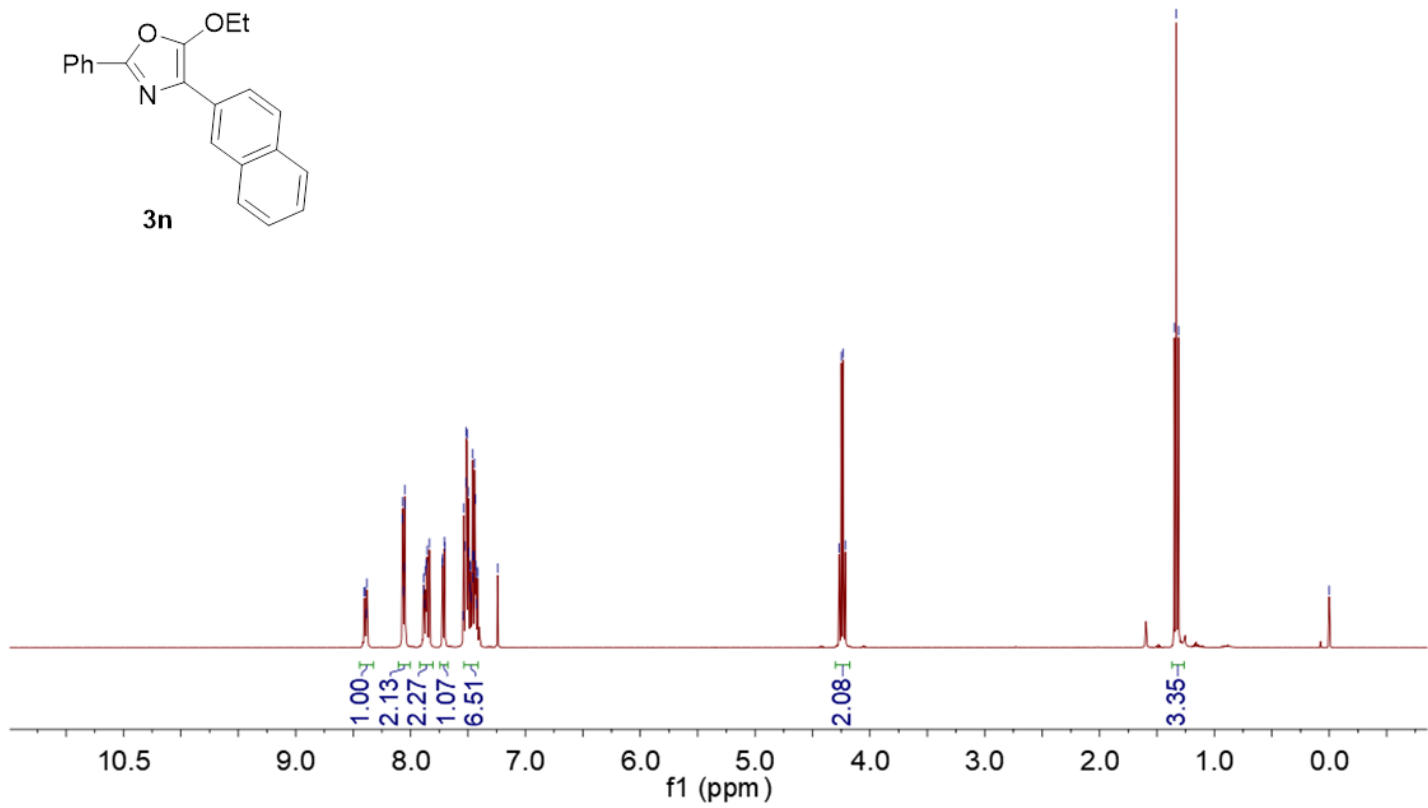

${ }^{13} \mathrm{C}$ NMR of $3 \mathbf{n}\left(100 \mathrm{MHz}, \mathrm{CDCl}_{3}\right)$

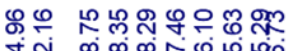

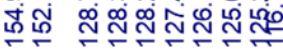

กิํำ용

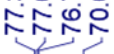

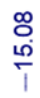
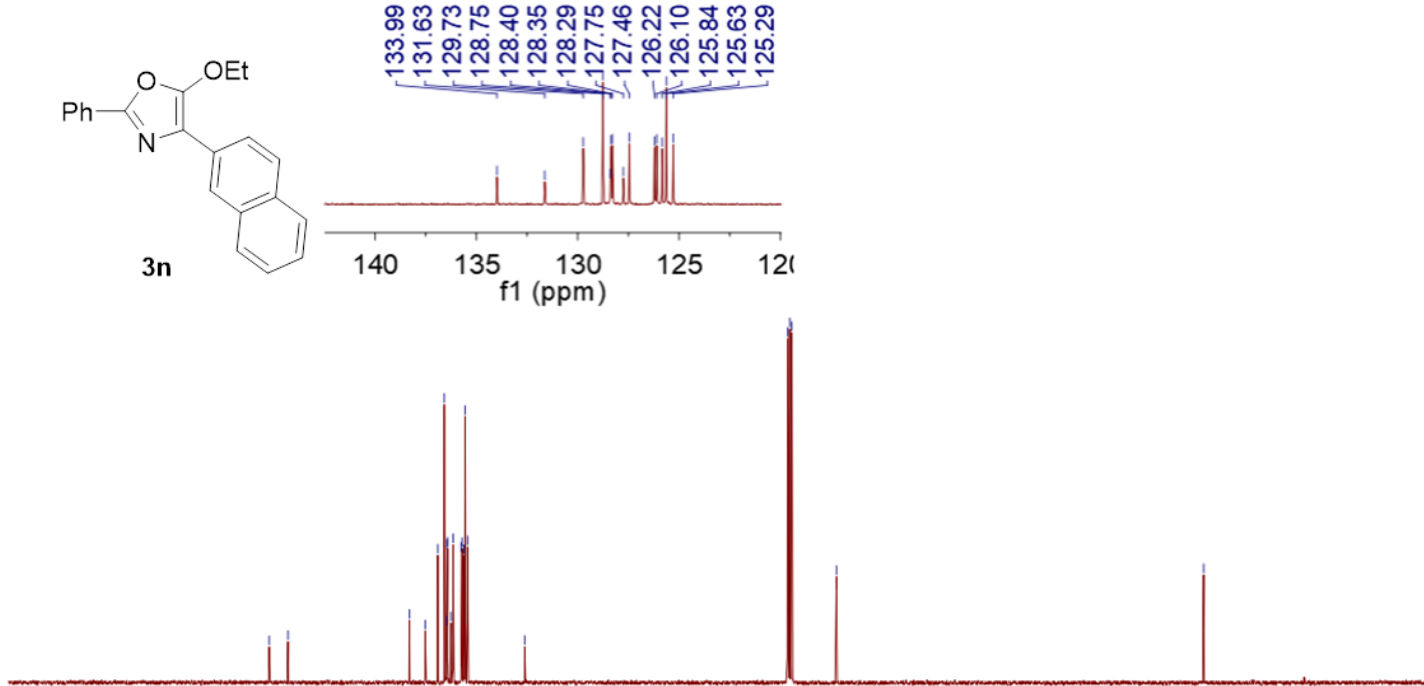

190

170

150

130

110

$\underset{91(\mathrm{ppm})}{90}$

$\begin{array}{llllllll}70 & 60 & 50 & 40 & 30 & 20 & 10 & 0\end{array}$ 
${ }^{1} \mathrm{H}$ NMR of $30\left(400 \mathrm{MHz}, \mathrm{CDCl}_{3}\right)$
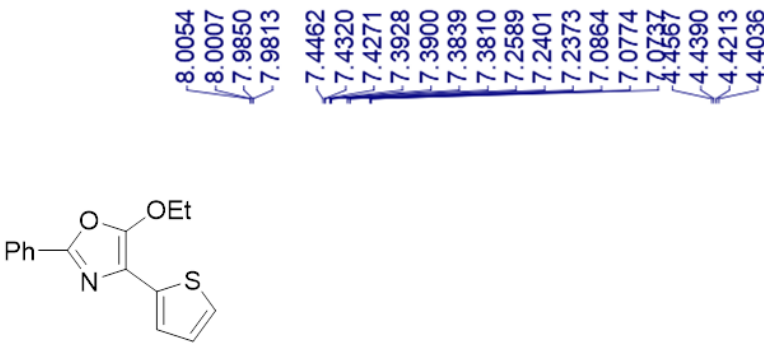

30

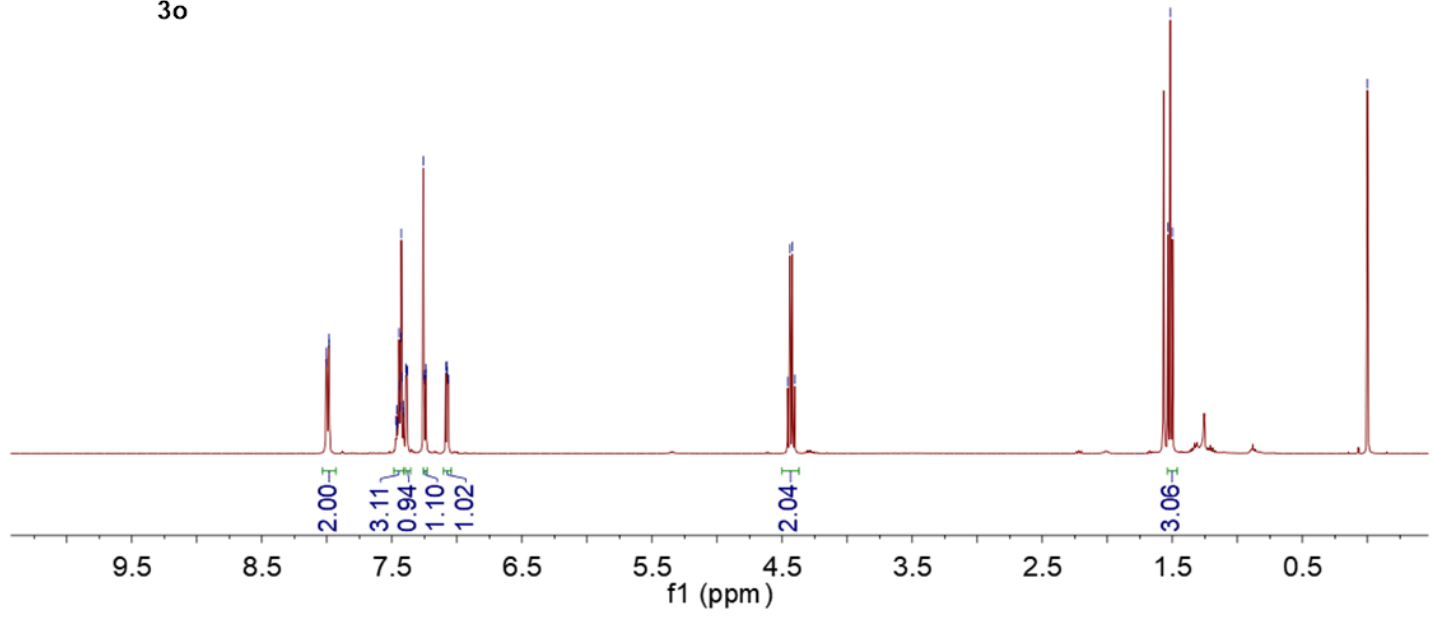

${ }^{13} \mathrm{C}$ NMR of $30\left(100 \mathrm{MHz}, \mathrm{CDCl}_{3}\right)$

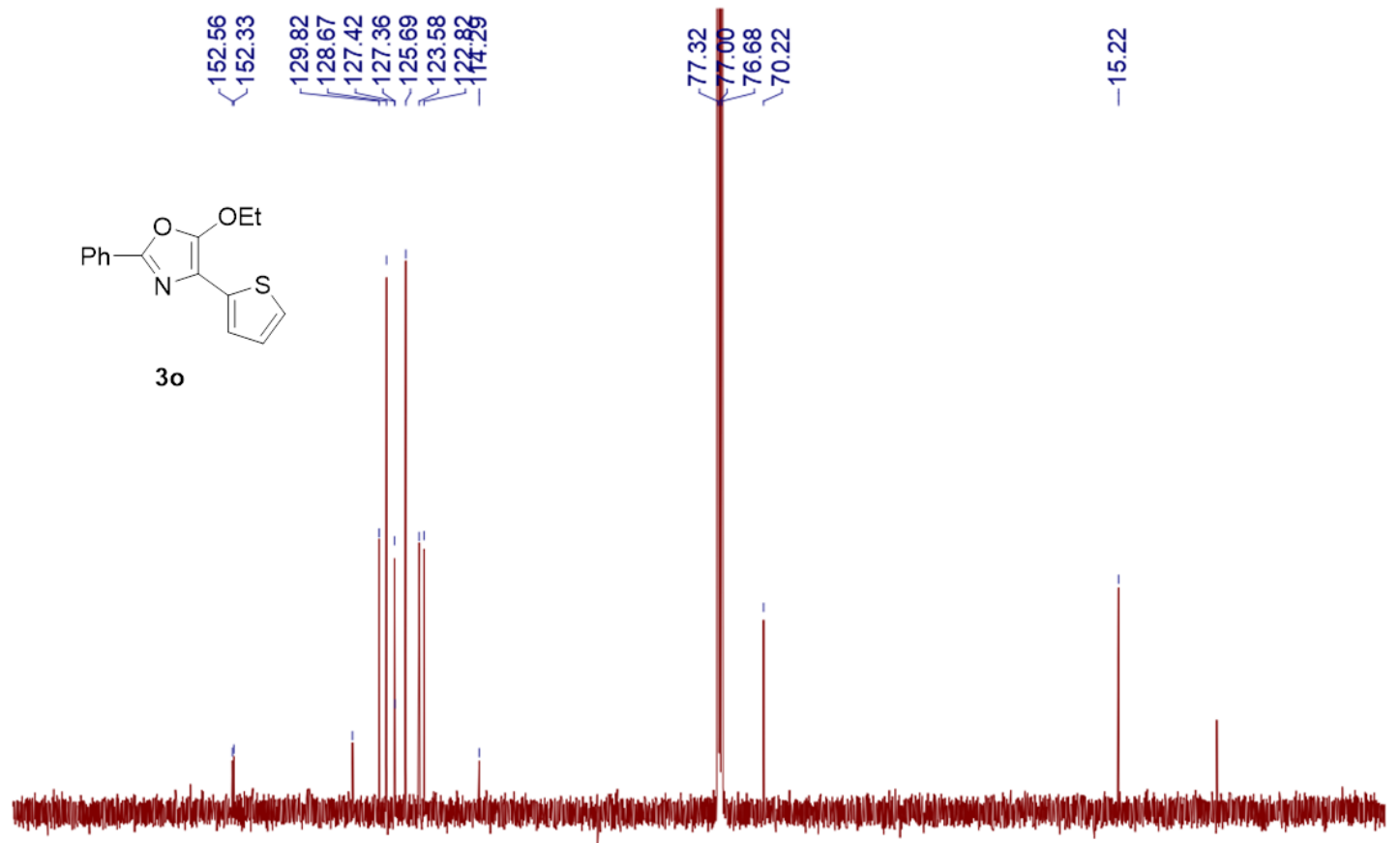

170

150

130

110

908070 
${ }^{1} \mathrm{H}$ NMR of 3p $\left(400 \mathrm{MHz}, \mathrm{CDCl}_{3}\right)$

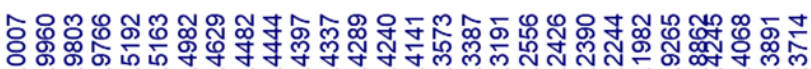

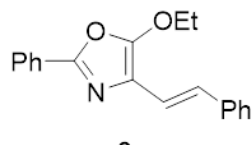

$3 p$

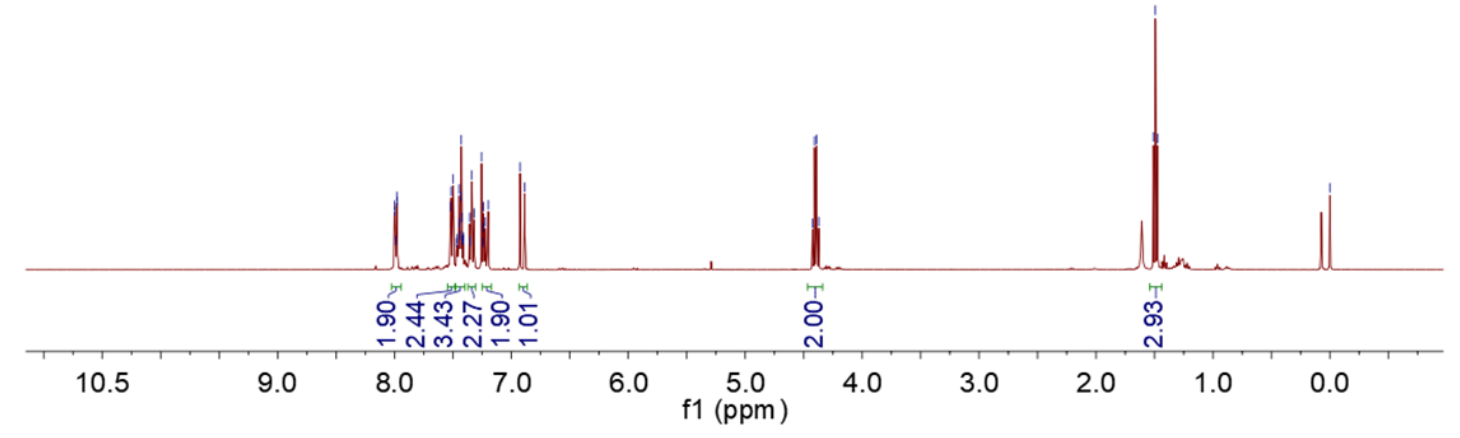

${ }^{13} \mathrm{C}$ NMR of 3p $\left(100 \mathrm{MHz}, \mathrm{CDCl}_{3}\right)$

นุด

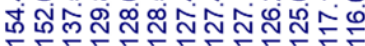

๙ู่

송ํㅇ

음

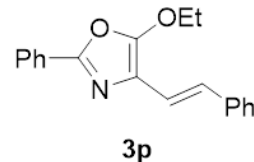

$3 p$

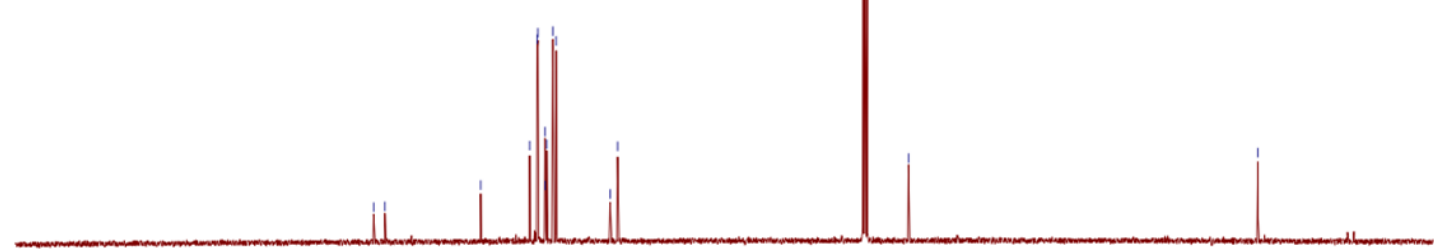

210

$190 \quad 170$

150

130

110

1 1 (ppm) 
${ }^{1} \mathrm{H}$ NMR of $\mathbf{3 q}\left(400 \mathrm{MHz}, \mathrm{CDCl}_{3}\right)$

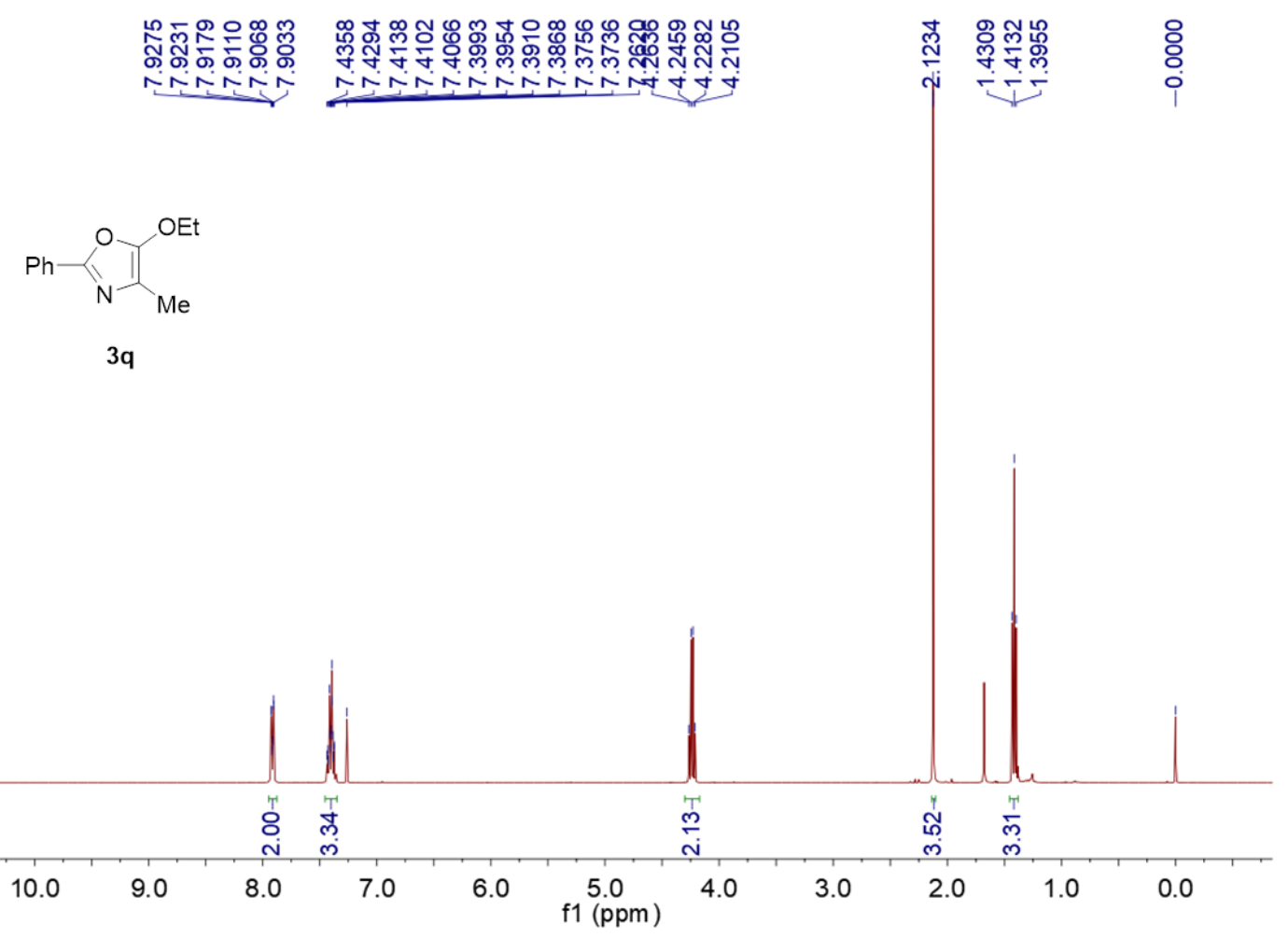

${ }^{13} \mathrm{C}$ NMR of 3q $\left(100 \mathrm{MHz}, \mathrm{CDCl}_{3}\right)$

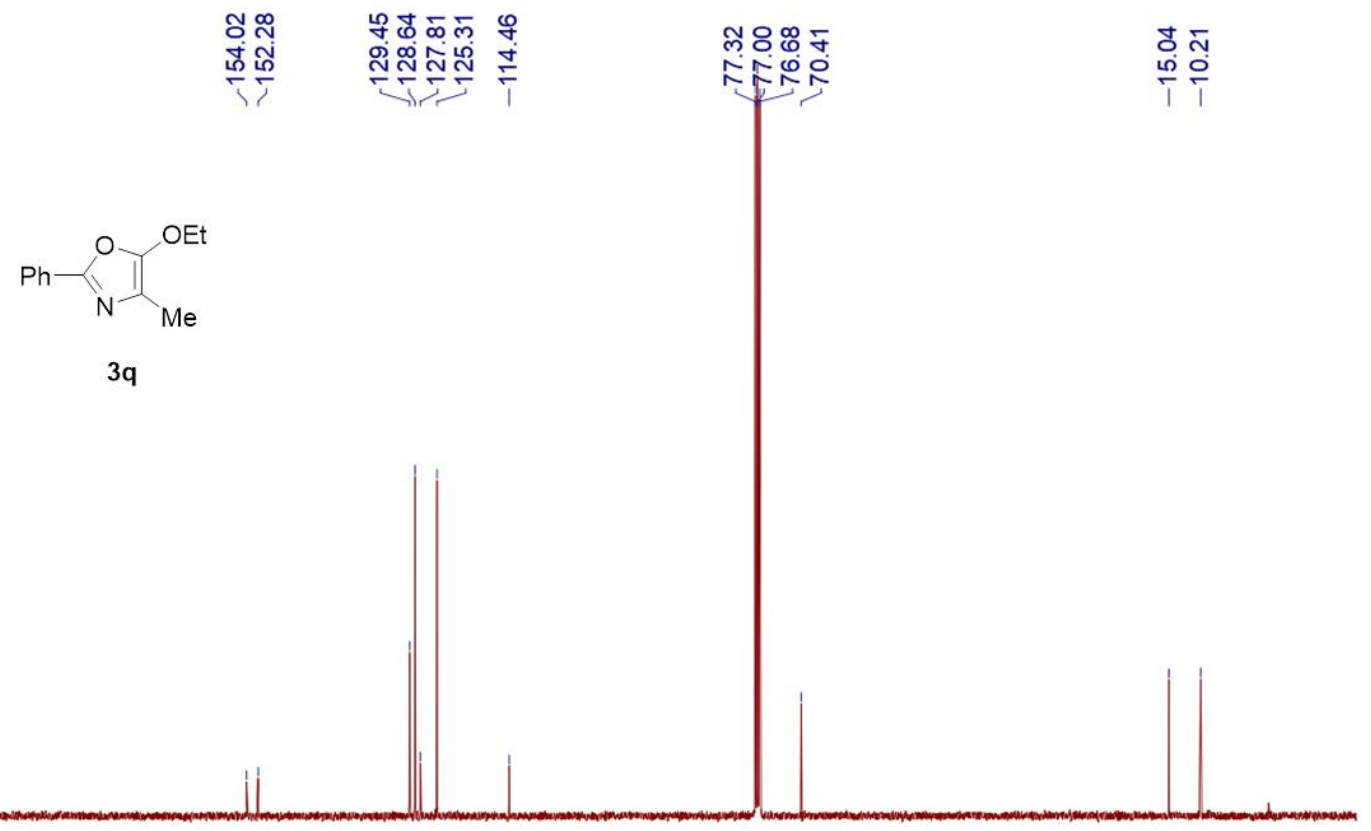

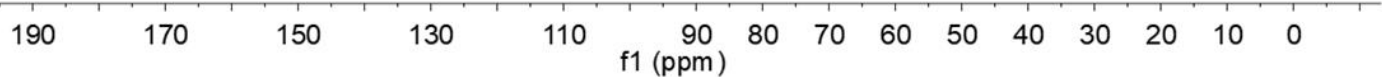


${ }^{1} \mathrm{H} \mathrm{NMR}$ of $\mathbf{3 r}\left(400 \mathrm{MHz}, \mathrm{CDCl}_{3}\right)$
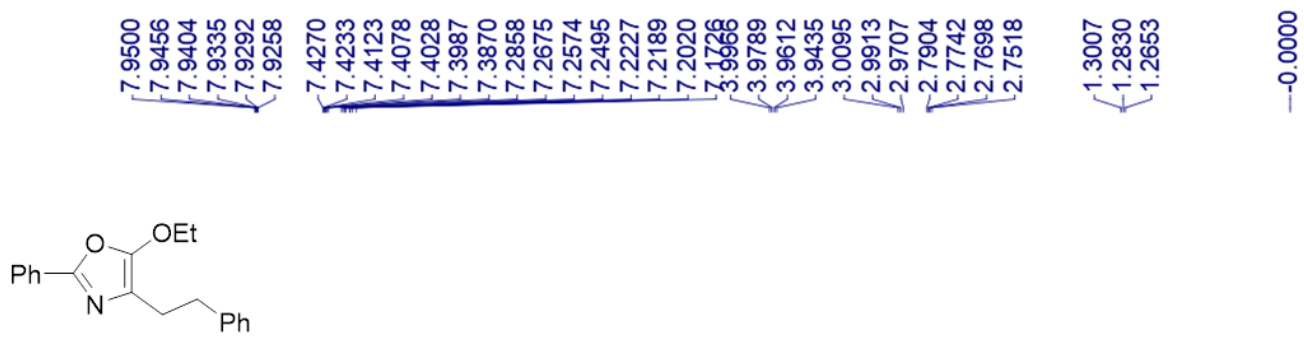

$3 r$

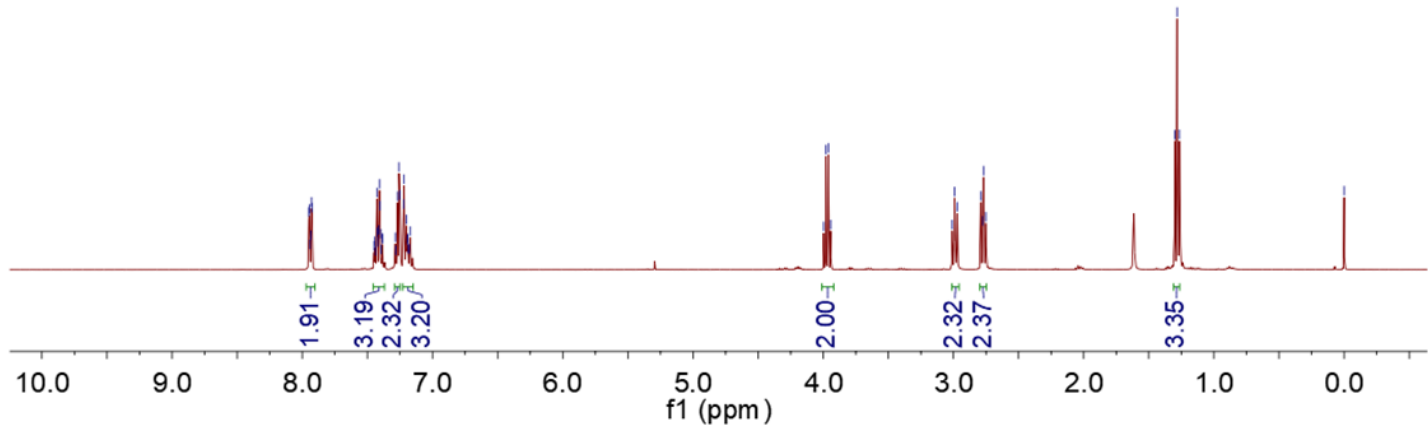

${ }^{13} \mathrm{C}$ NMR of $3 \mathbf{r}\left(100 \mathrm{MHz}, \mathrm{CDCl}_{3}\right)$

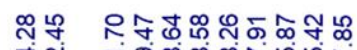

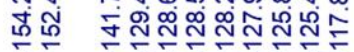

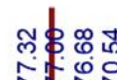

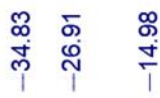

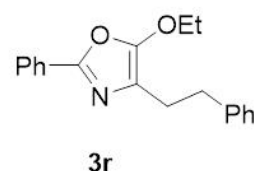

$3 r$

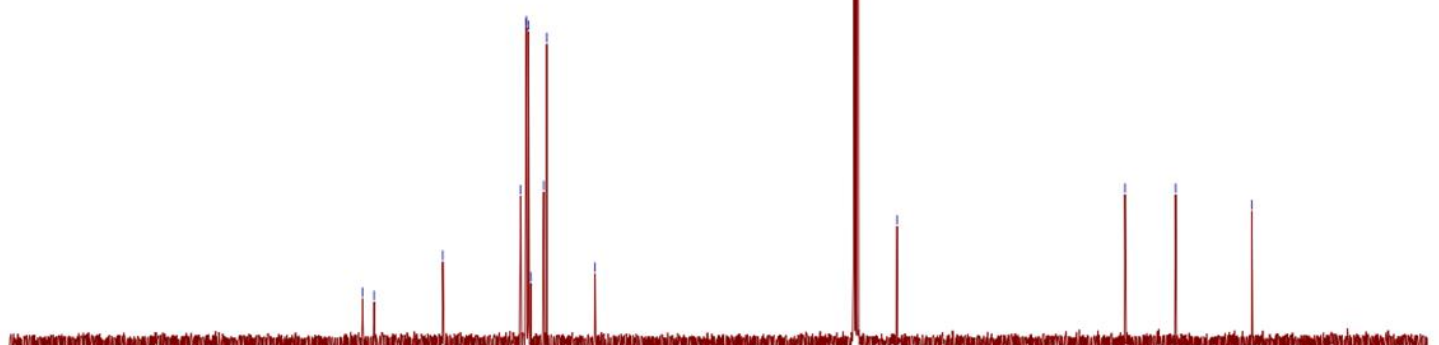

190

170

150

130

90

$\begin{array}{lllllllll}80 & 70 & 60 & 50 & 40 & 30 & 20 & 10 & 0\end{array}$ 
${ }^{1} \mathrm{H}$ NMR of $3 \mathbf{s}\left(400 \mathrm{MHz}, \mathrm{CDCl}_{3}\right)$

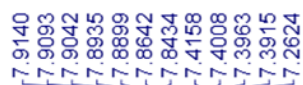

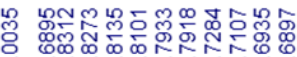

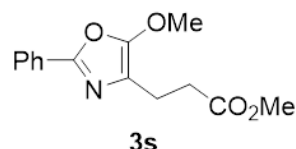

$3 \mathrm{~s}$

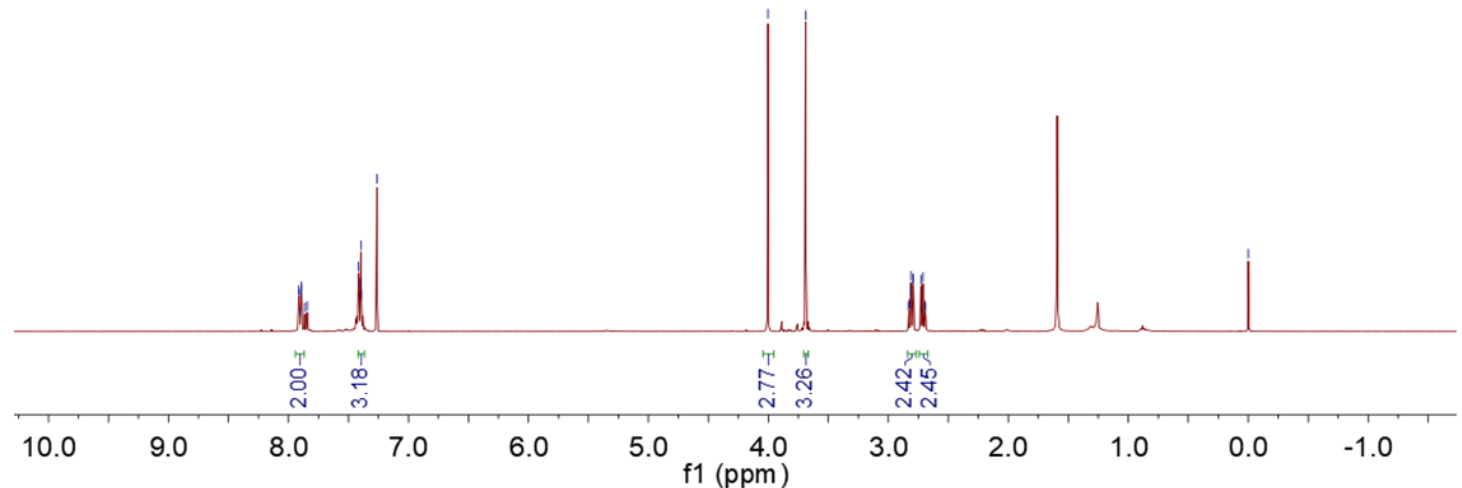

${ }^{13} \mathrm{C}$ NMR of $3 \mathbf{s}\left(100 \mathrm{MHz}, \mathrm{CDCl}_{3}\right)$

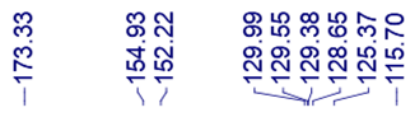

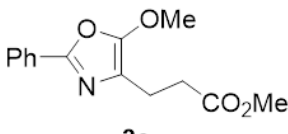

$3 s$
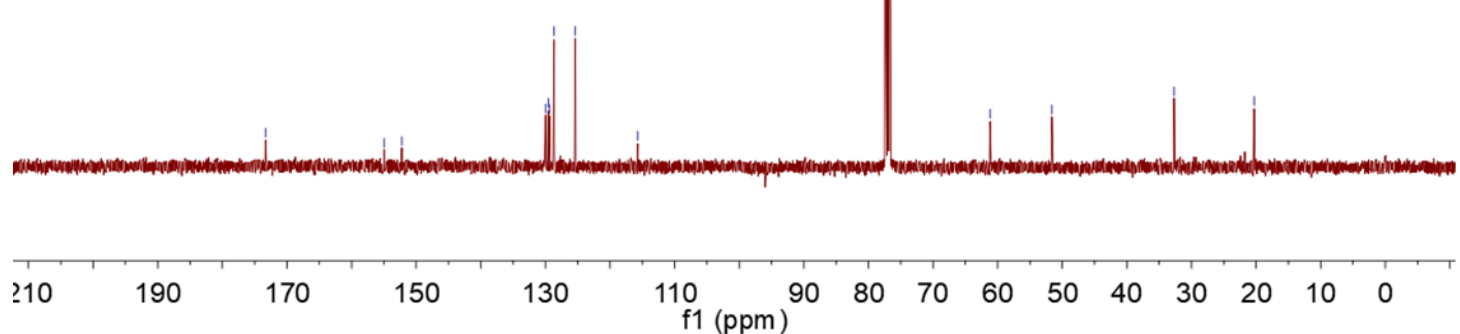
${ }^{1} \mathrm{H}$ NMR of $\mathbf{3 t}\left(400 \mathrm{MHz}, \mathrm{CDCl}_{3}\right)$

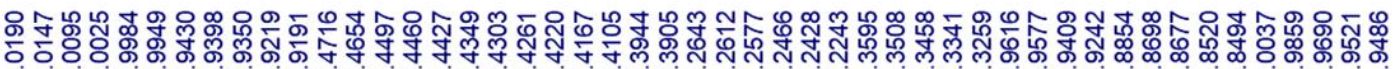
o
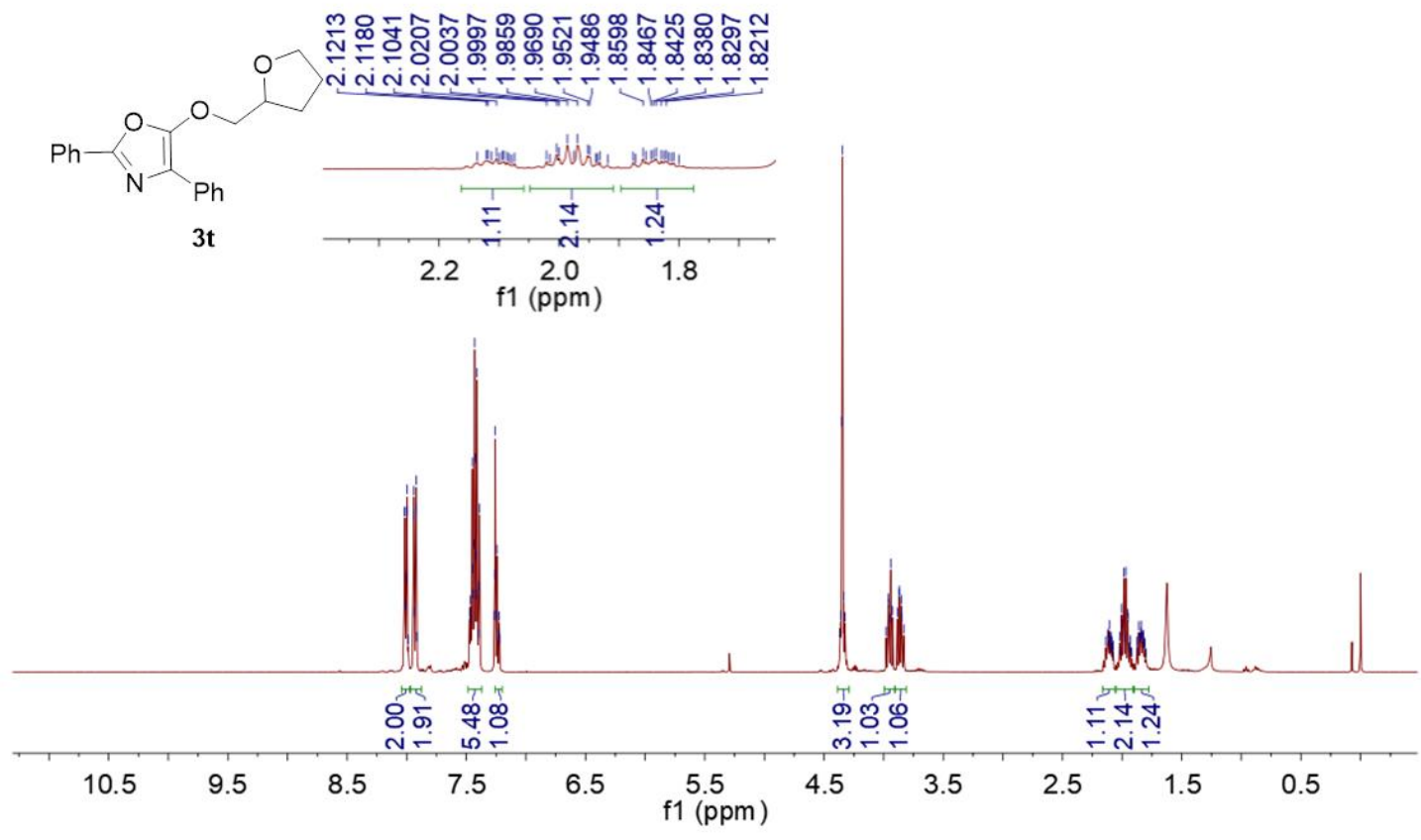

${ }^{13} \mathrm{C}$ NMR of $3 t\left(100 \mathrm{MHz}, \mathrm{CDCl}_{3}\right)$

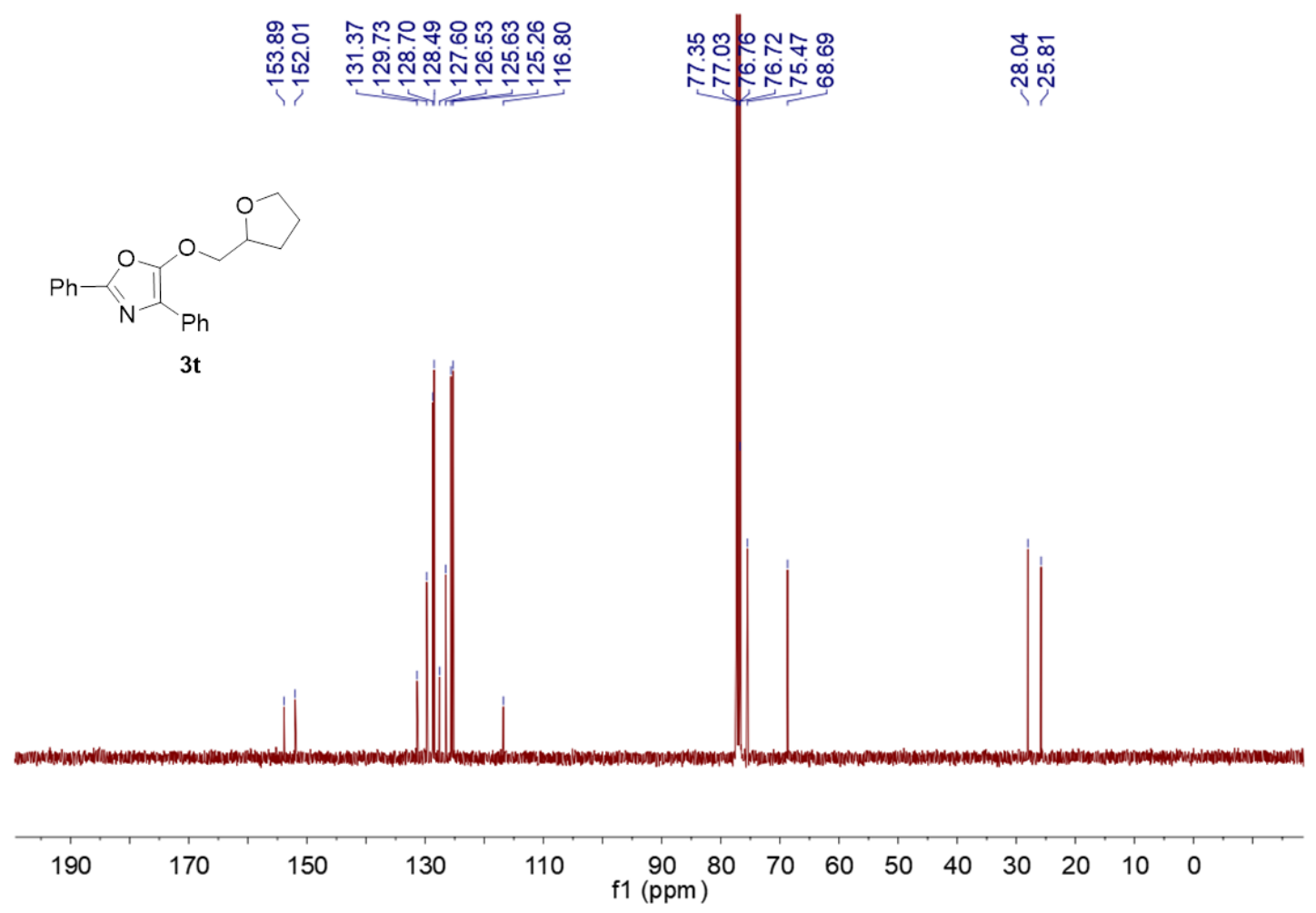


${ }^{1} \mathrm{H}$ NMR of $3 \mathbf{u}\left(400 \mathrm{MHz}, \mathrm{CDCl}_{3}\right)$

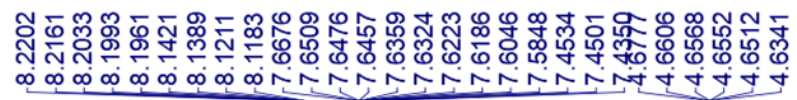

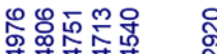

$\infty \infty_{\infty} \infty \infty_{\infty}$

ָิ

$\overbrace{\mathrm{Ph}}^{\mathrm{O}}$

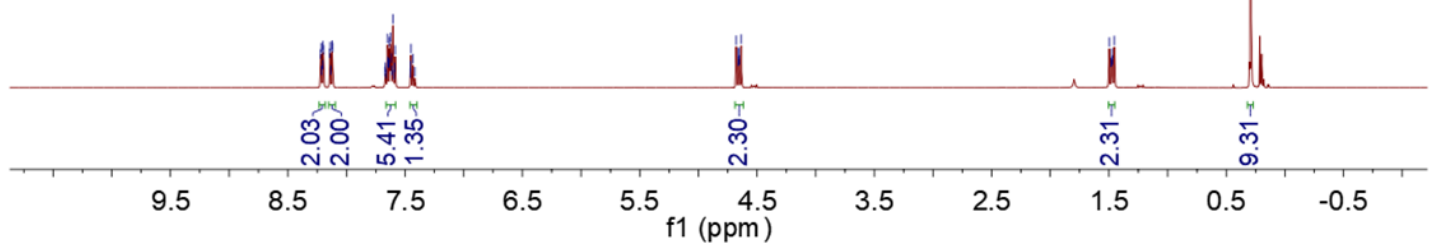

${ }^{13} \mathrm{C}$ NMR of $3 \mathbf{u}\left(100 \mathrm{MHz}, \mathrm{CDCl}_{3}\right)$

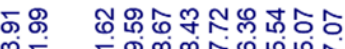

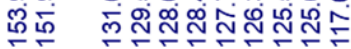

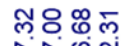

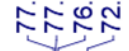

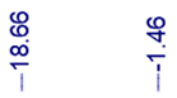



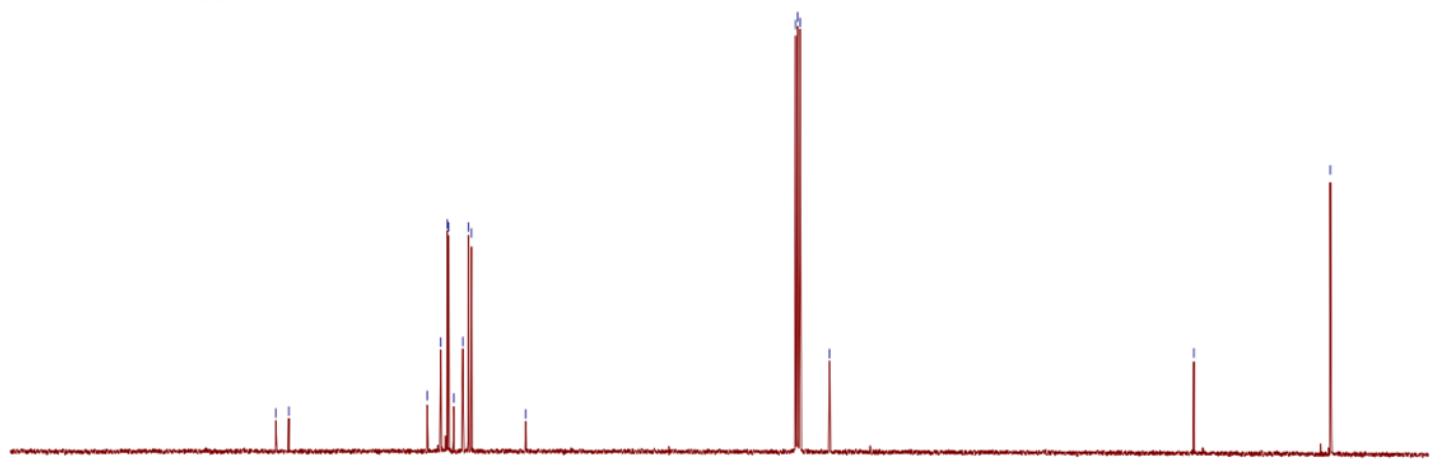

190

170

150

130

110

9080

$\begin{array}{llllllll}60 & 50 & 40 & 30 & 20 & 10 & 0\end{array}$

SI-50 
${ }^{1} \mathrm{H}$ NMR of $3 \mathbf{v}\left(400 \mathrm{MHz}\right.$, DMSO- $\left.d_{6}\right)$
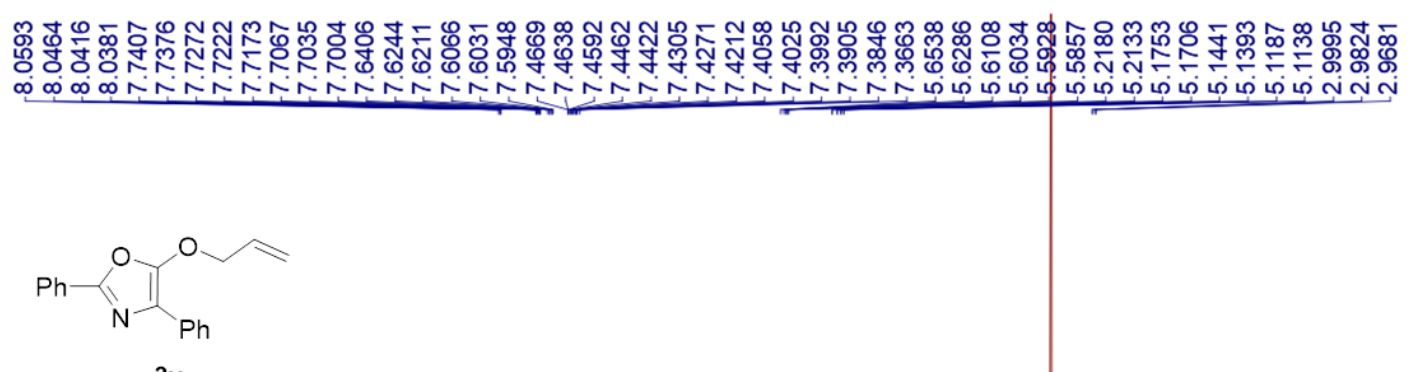

$3 v$

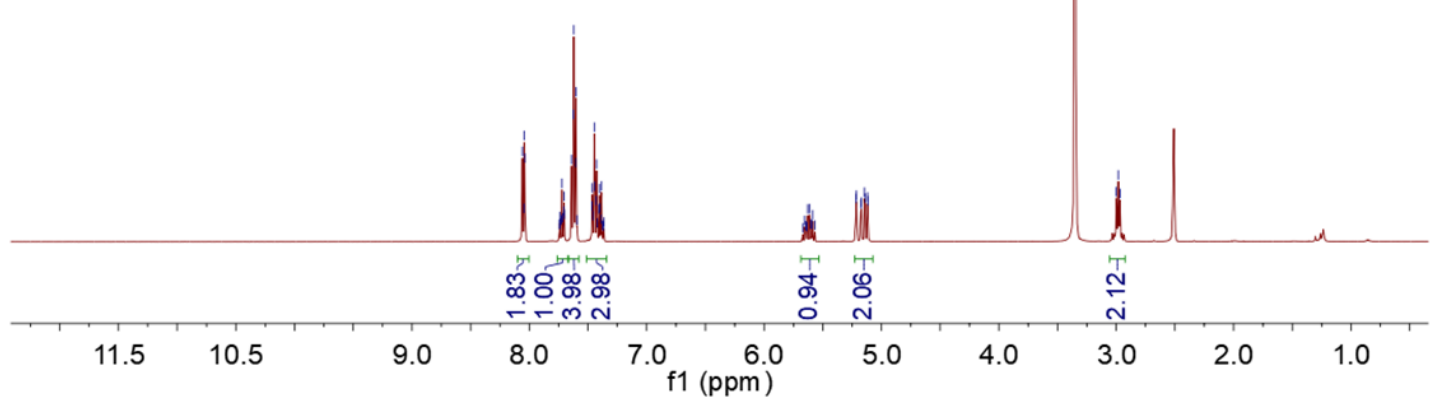

${ }^{13} \mathrm{C}$ NMR of $3 \mathbf{v}\left(100 \mathrm{MHz}, \mathrm{DMSO}-d_{6}\right)$

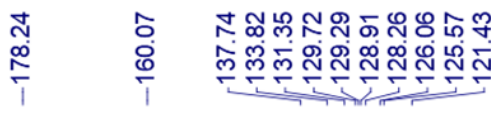

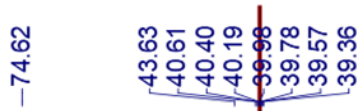

Ph

$3 v$

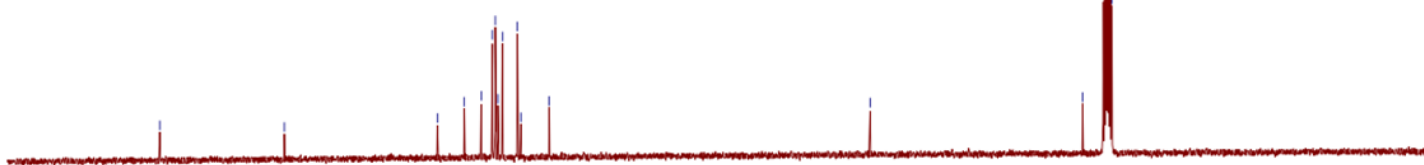

190

150

130

110

$\begin{array}{lllllllll}80 & 70 & 60 & 50 & 40 & 30 & 20 & 10 & 0\end{array}$ 
${ }^{1} \mathrm{H}$ NMR of 3w (400 MHz, DMSO- $d_{6}$ )

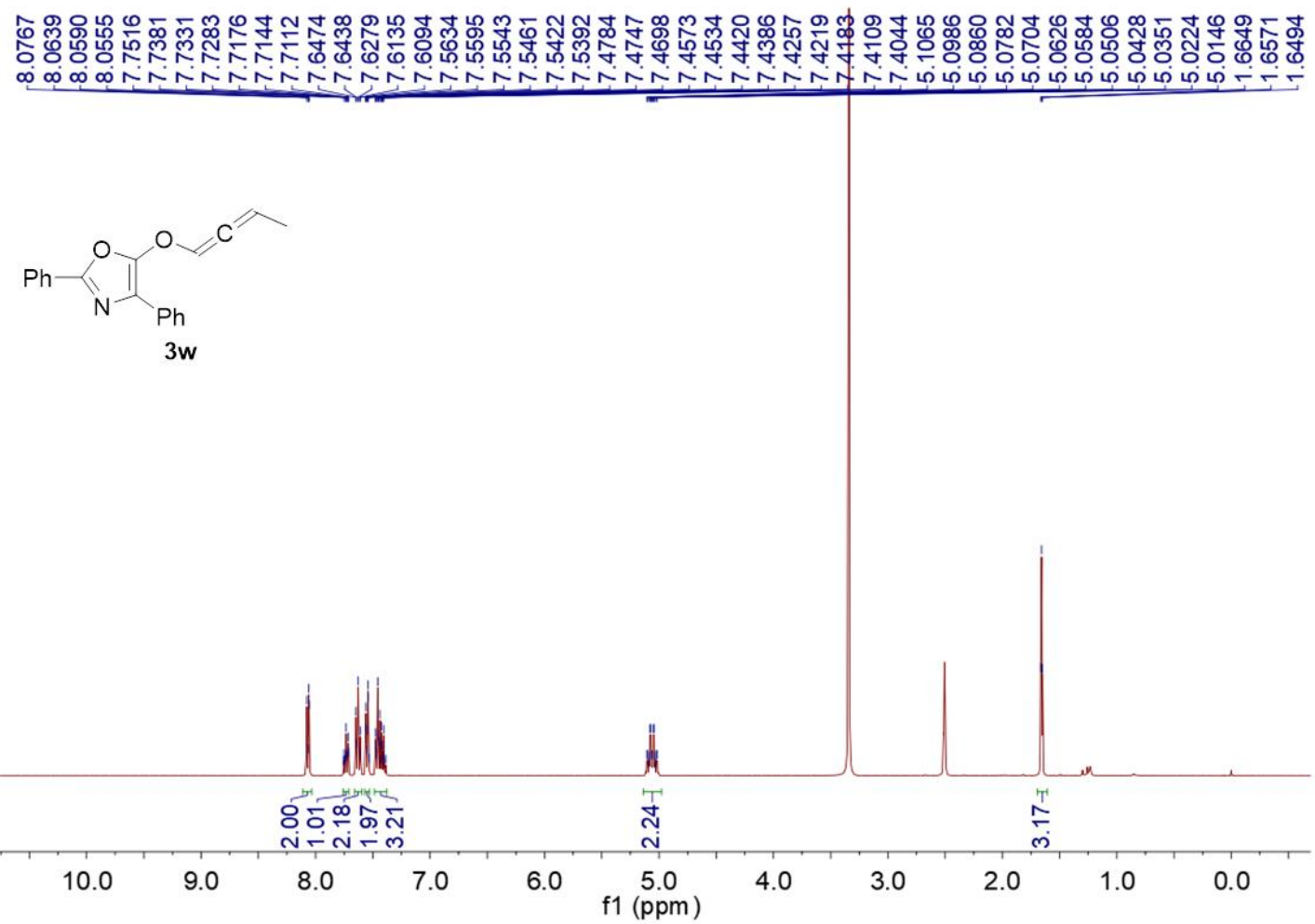

${ }^{13} \mathrm{C}$ NMR of 3w (100 MHz, DMSO- $\left.d_{6}\right)$

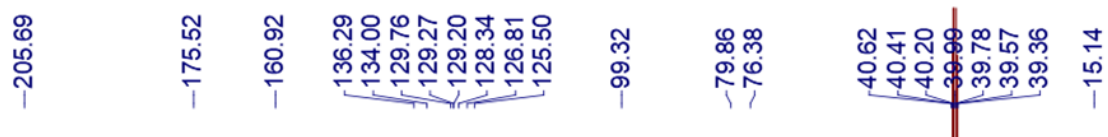<smiles>C/C=C\COc1oc(-c2ccccc2)nc1-c1ccccc1</smiles>

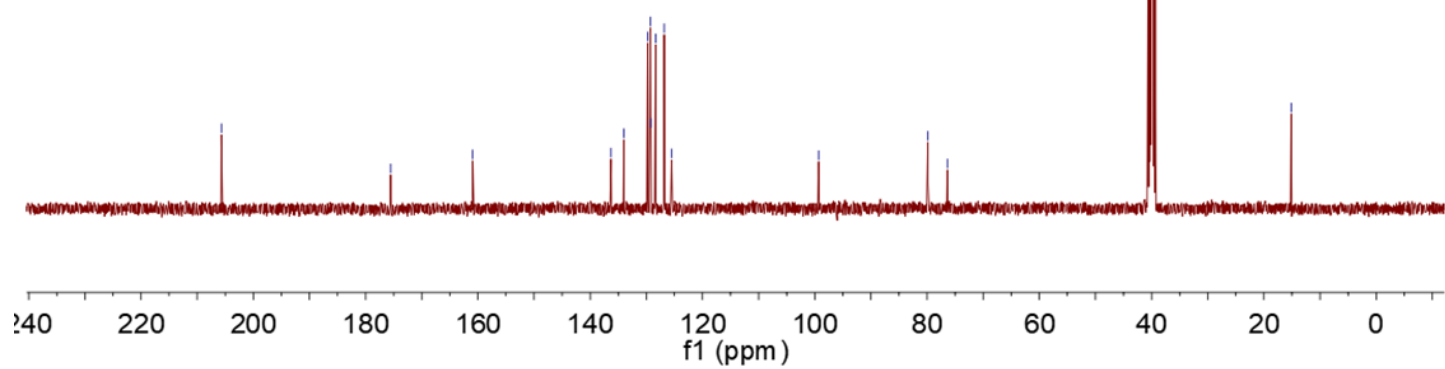


${ }^{1} \mathrm{H}$ NMR of $3 \mathbf{x}\left(400 \mathrm{MHz}, \mathrm{CDCl}_{3}\right)$

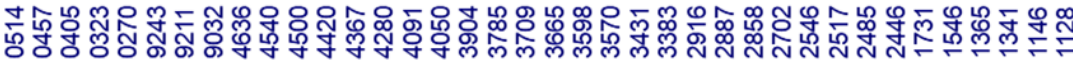

o 0 o m on<smiles>c1ccc(Oc2nc(-c3ccccc3)oc2-c2ccccc2)cc1</smiles>

$3 x$

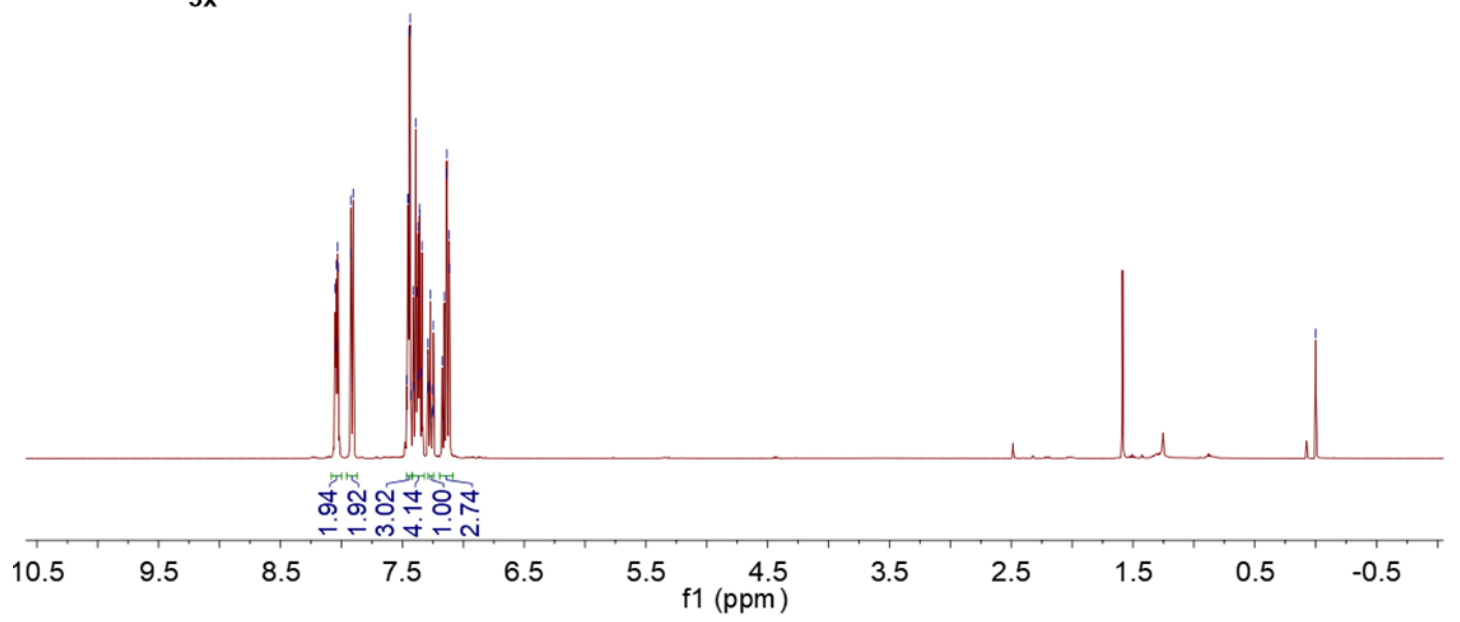

${ }^{13} \mathrm{C} \mathrm{NMR}$ of $3 \mathbf{x}\left(100 \mathrm{MHz}, \mathrm{CDCl}_{3}\right)$

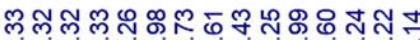

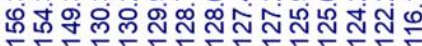

$\overbrace{\mathrm{Nh}}^{\mathrm{O}_{\mathrm{Ph}}}$

$3 x$

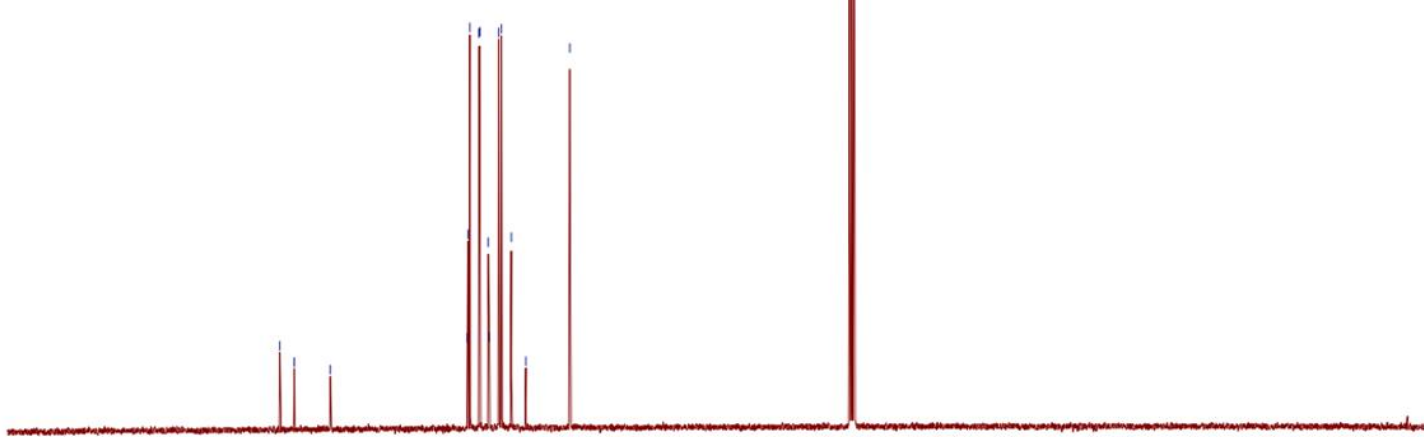

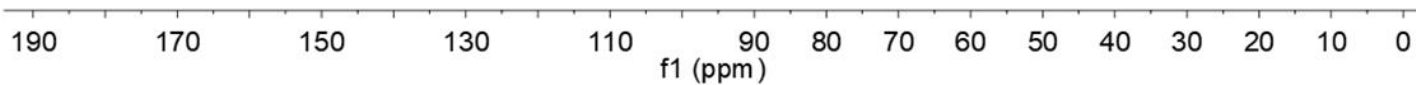


${ }^{1} \mathrm{H}$ NMR of $\mathbf{3 y}\left(400 \mathrm{MHz}, \mathrm{CDCl}_{3}\right)$

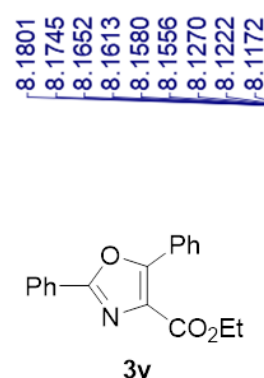

$3 y$

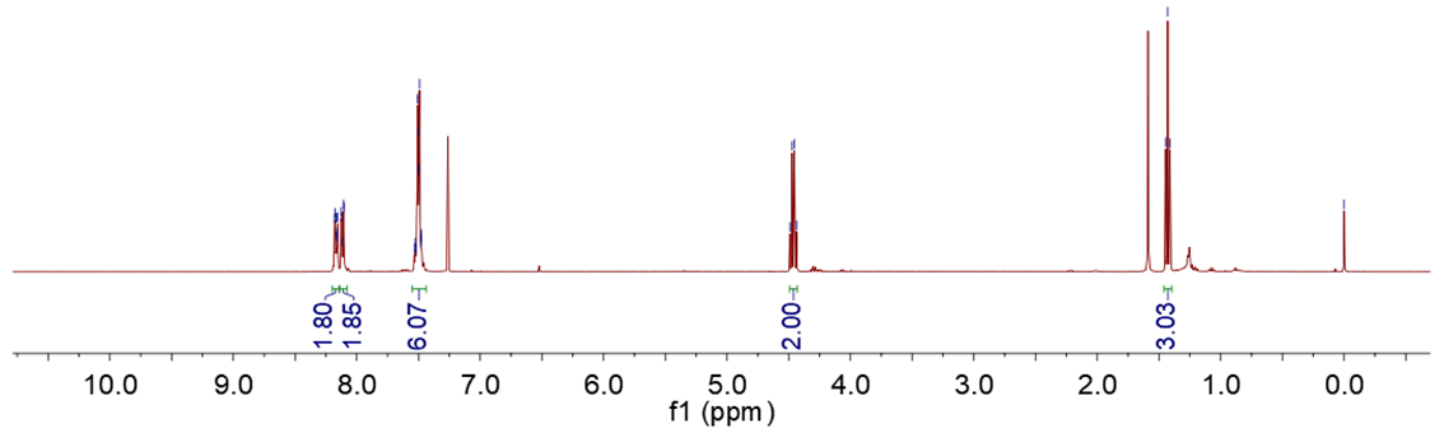

${ }^{13} \mathrm{C}$ NMR of $\mathbf{3 y}\left(100 \mathrm{MHz}, \mathrm{CDCl}_{3}\right)$
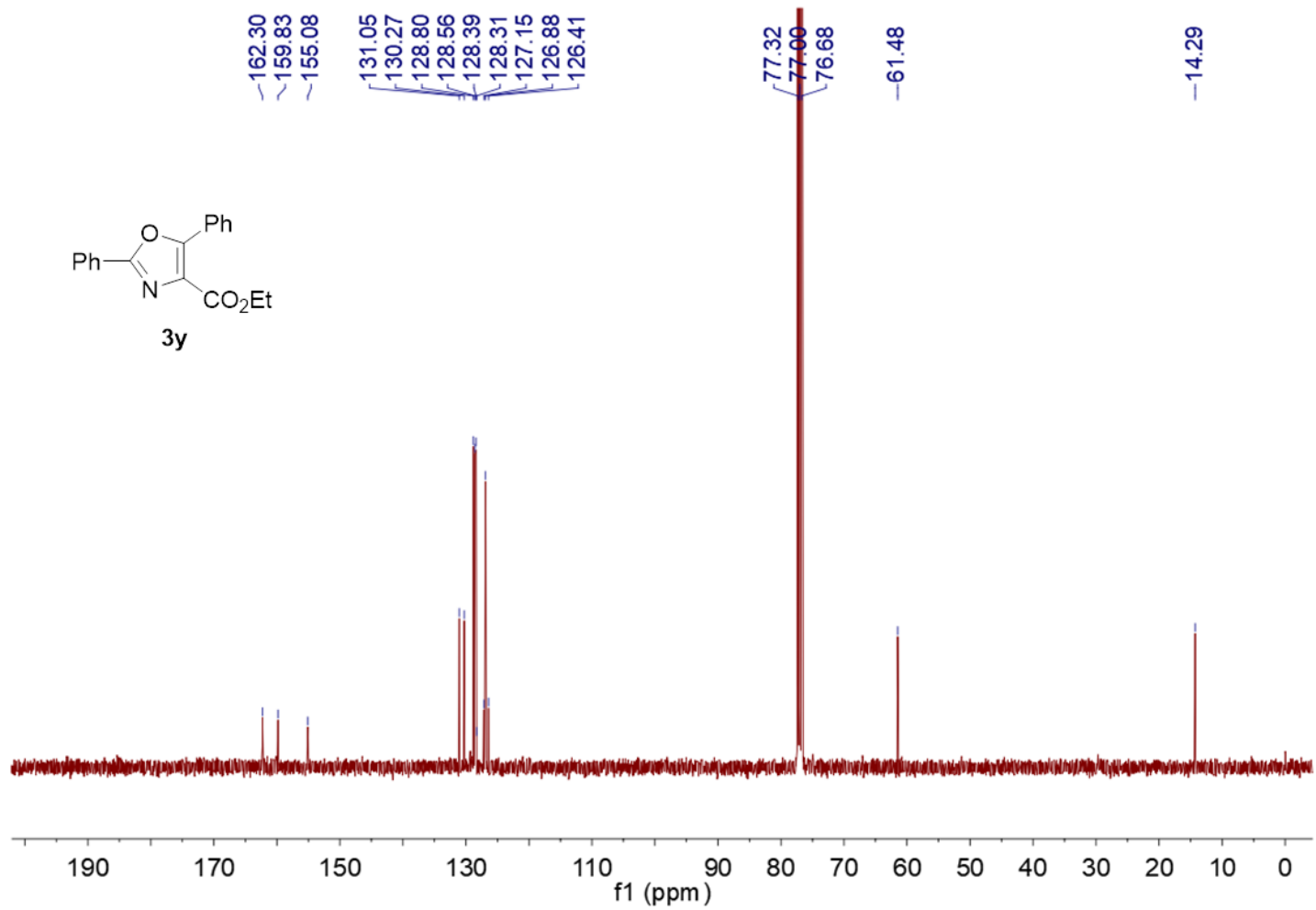
${ }^{1} \mathrm{H}$ NMR of $\mathbf{3 z}\left(400 \mathrm{MHz}, \mathrm{CDCl}_{3}\right)$

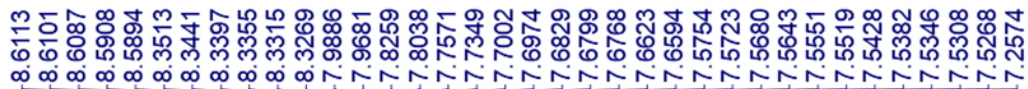

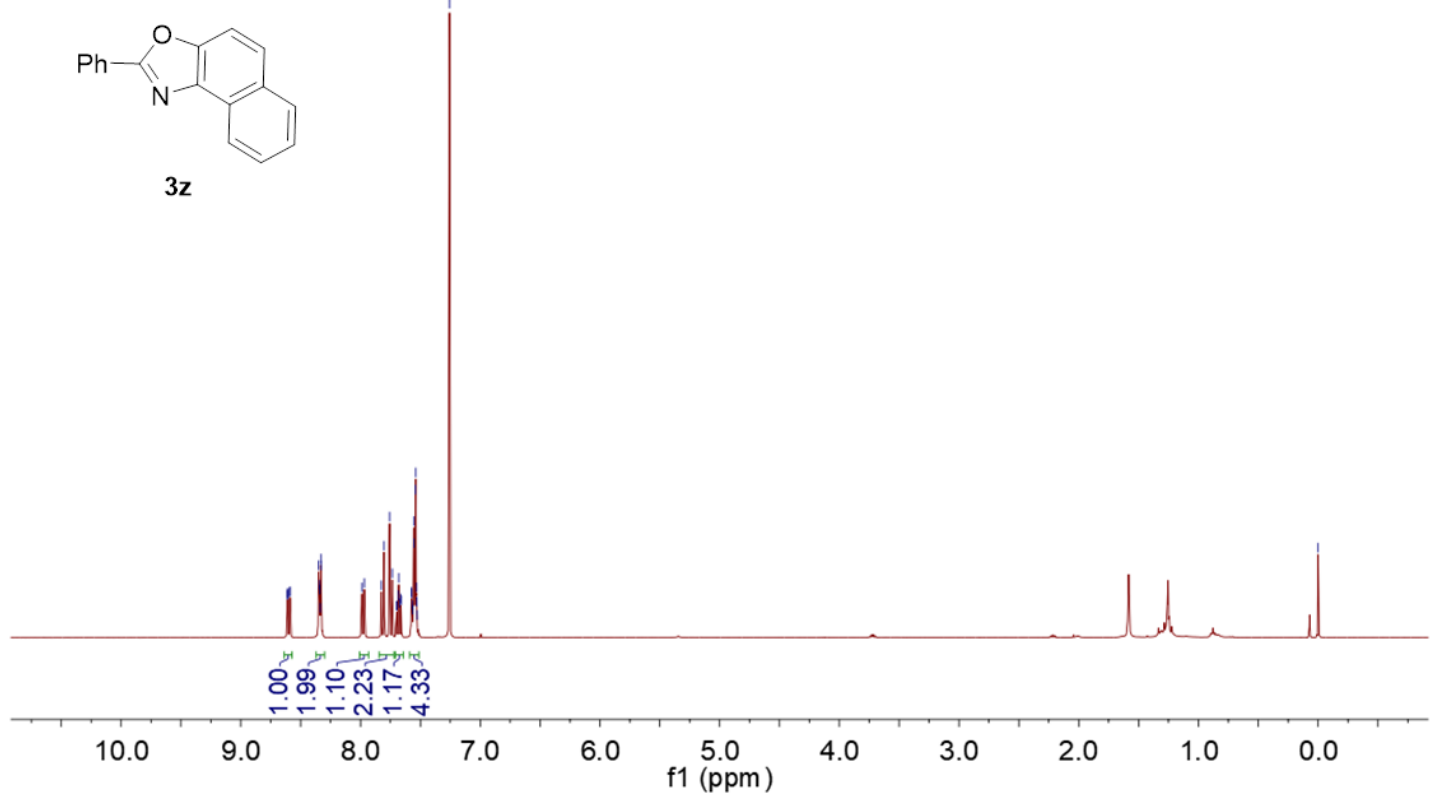

${ }^{13} \mathrm{C}$ NMR of $\mathbf{3 z}\left(100 \mathrm{MHz}, \mathrm{CDCl}_{3}\right)$

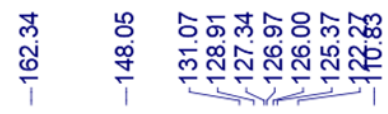

$\uparrow$ i

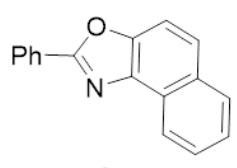

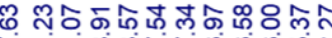

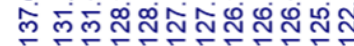

$3 z$

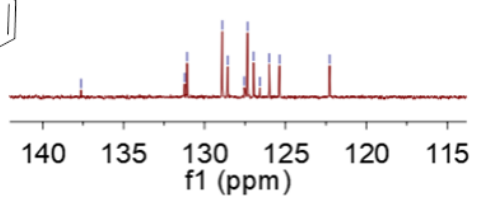

190

170

150

130

110

$90 \quad 80 \quad 70 \quad 60$

f1 (ppm) 
${ }^{1} \mathrm{H}$ NMR of $\mathbf{4 a}\left(400 \mathrm{MHz}, \mathrm{CDCl}_{3}\right)$
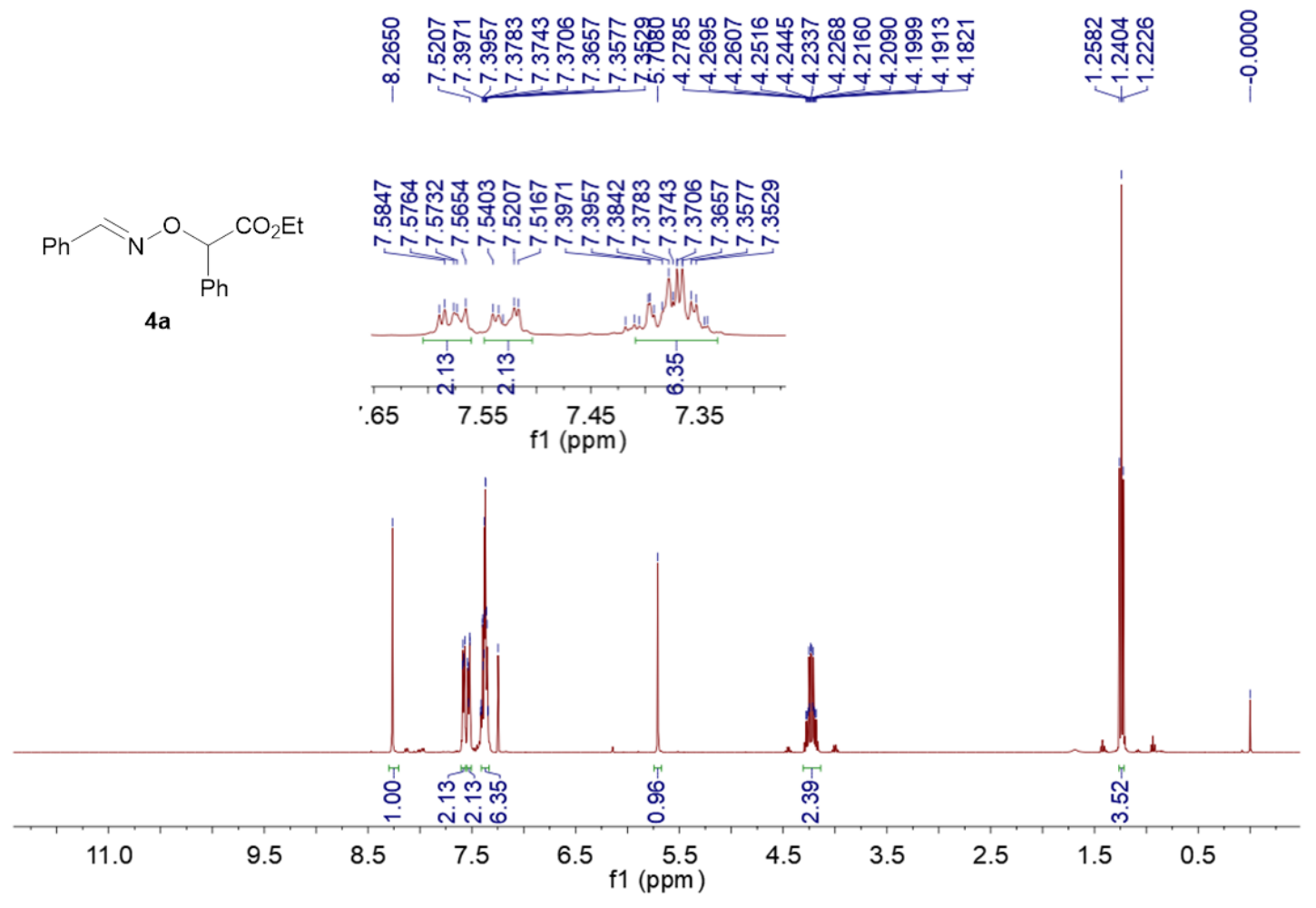

${ }^{13} \mathrm{C}$ NMR of $4 \mathbf{a}\left(100 \mathrm{MHz}, \mathrm{CDCl}_{3}\right)$

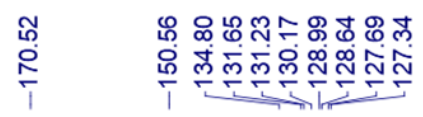

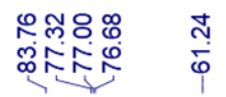

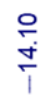

$\mathrm{Ph} \sim \mathrm{N}_{\mathrm{Ph}} \mathrm{CO}_{2} \mathrm{Et}$

4a

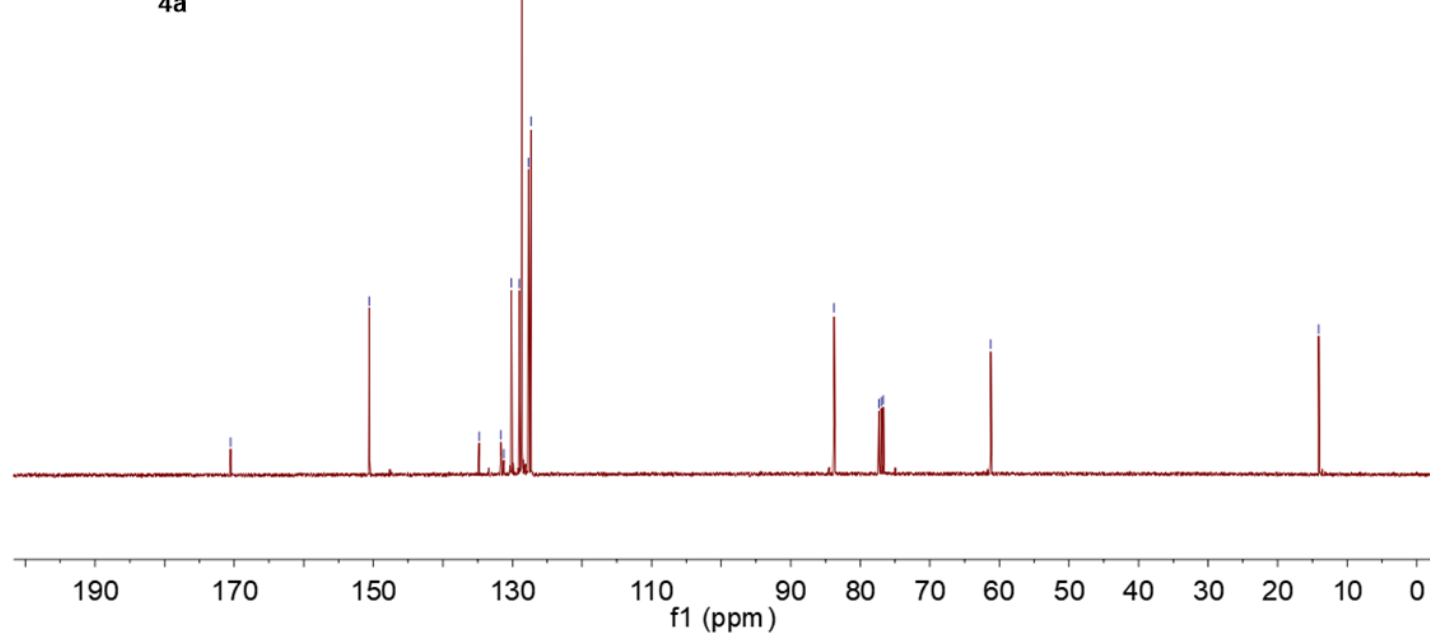


${ }^{1} \mathrm{H}$ NMR of $\mathbf{4 b}\left(400 \mathrm{MHz}, \mathrm{CDCl}_{3}\right)$

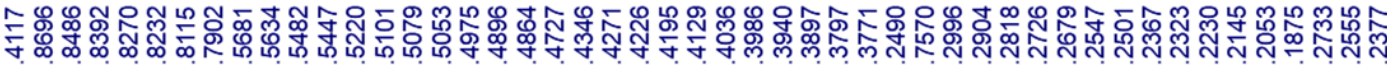

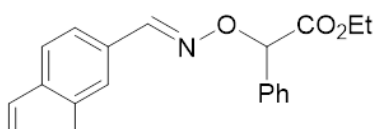

4b

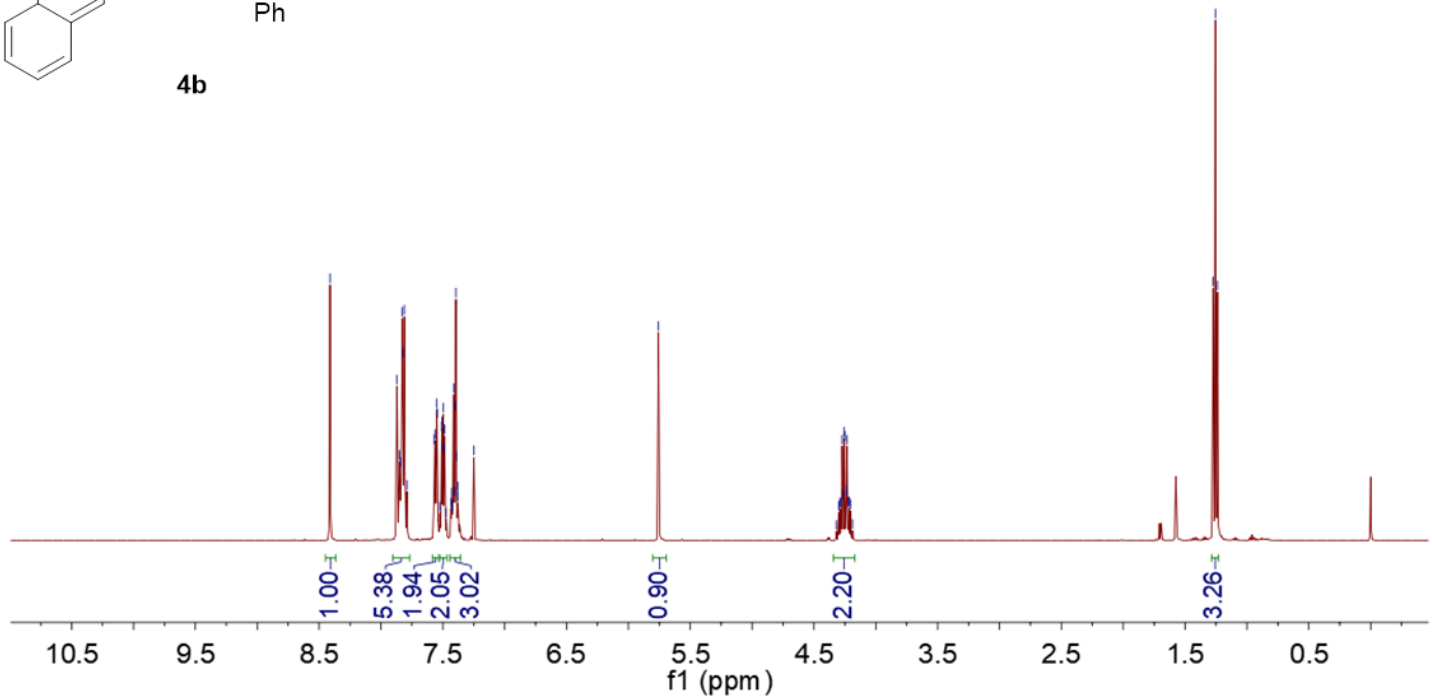

${ }^{13} \mathrm{C}$ NMR of $4 \mathbf{b}\left(100 \mathrm{MHz}, \mathrm{CDCl}_{3}\right)$

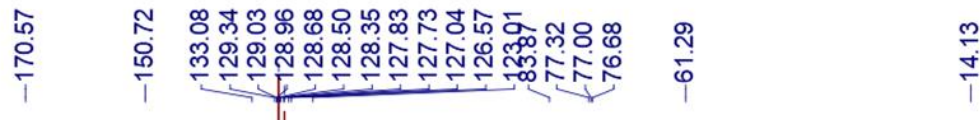

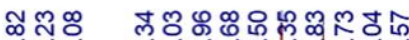

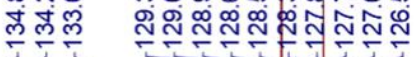

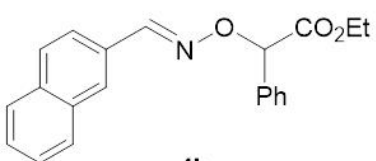

$4 b$

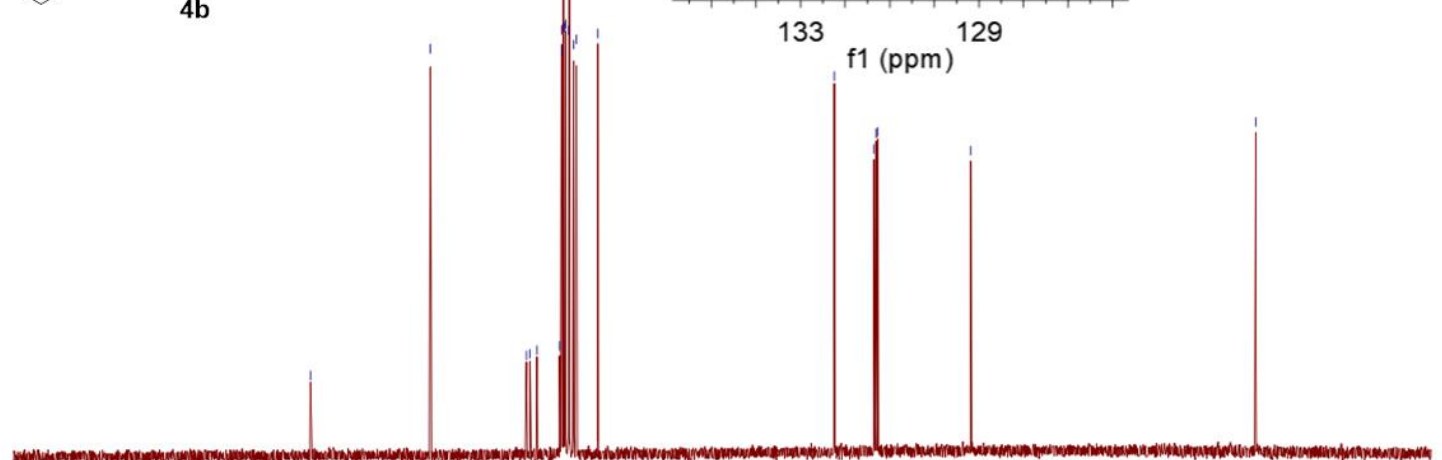

200

180

160

140

120

(ppm)

80

60

40

$20 \quad 0$ 
${ }^{1} \mathrm{H}$ NMR of $4 \mathbf{c}\left(400 \mathrm{MHz}, \mathrm{DMSO}-d_{6}\right)$

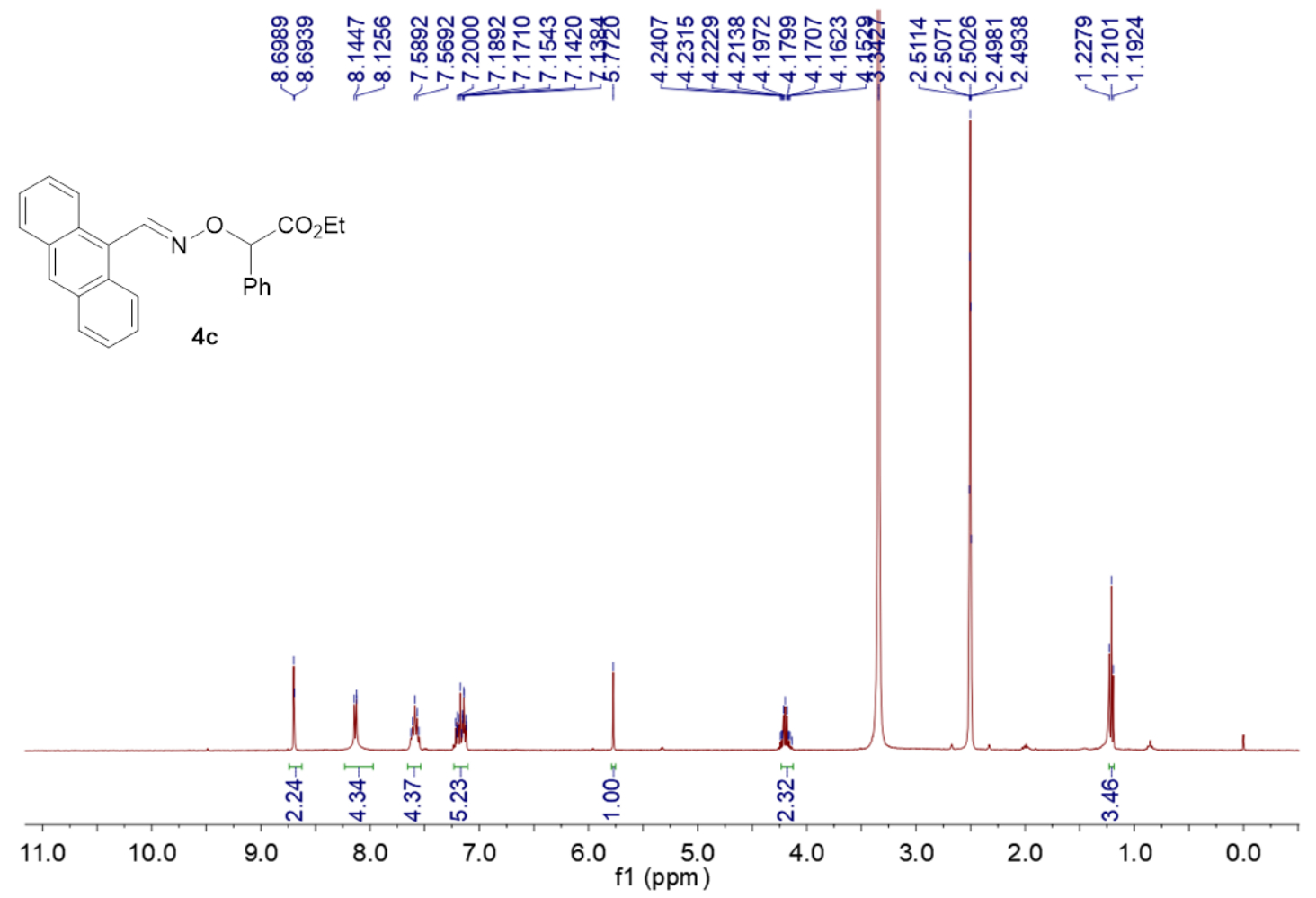

${ }^{13} \mathrm{C}$ NMR of $4 \mathbf{c}\left(100 \mathrm{MHz}\right.$, DMSO- $\left.d_{6}\right)$

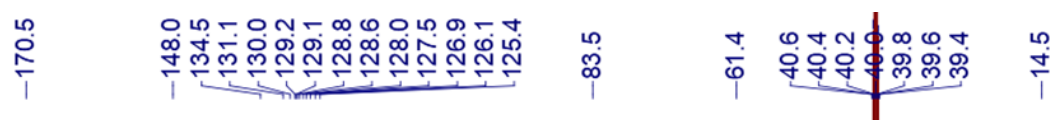

${ }_{4 c}^{\mathrm{Ph}} \mathrm{N}^{\mathrm{CO}_{2} \mathrm{Et}}$

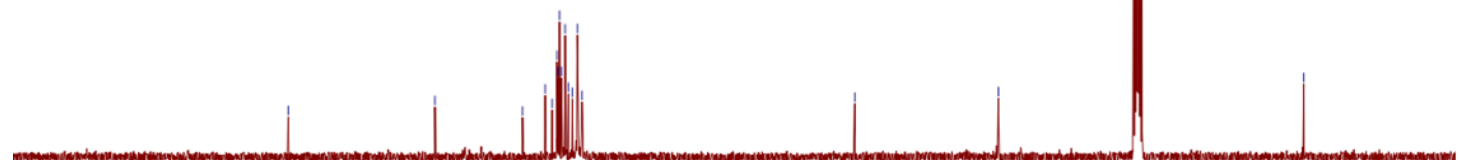

$\begin{array}{llllllllllllllllll}210 & 190 & 170 & 150 & 130 & \begin{array}{c}110 \\ \mathrm{f} 1(\mathrm{ppm})\end{array} & 90 & 80 & 70 & 60 & 50 & 40 & 30 & 20 & 10 & 0\end{array}$ 
${ }^{1} \mathrm{H}$ NMR of $4 \mathbf{d}\left(400 \mathrm{MHz}, \mathrm{CDCl}_{3}\right)$

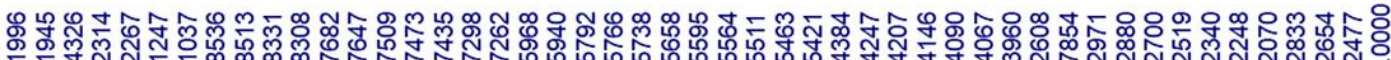

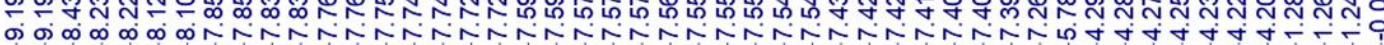

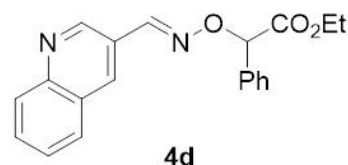

$4 d$

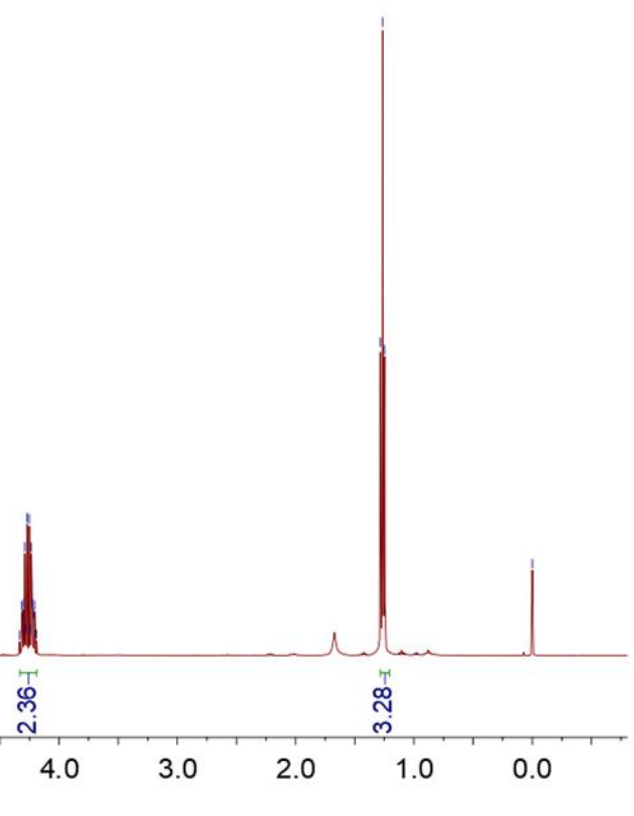

${ }^{13} \mathrm{C}$ NMR of $4 d\left(100 \mathrm{MHz}, \mathrm{CDCl}_{3}\right)$

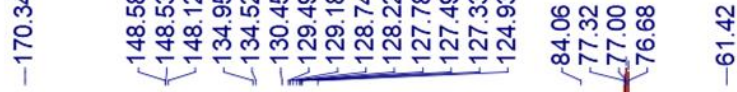

$\stackrel{m}{\stackrel{m}{\leftarrow}}$

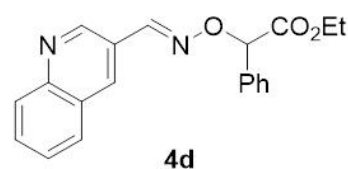

$4 d$

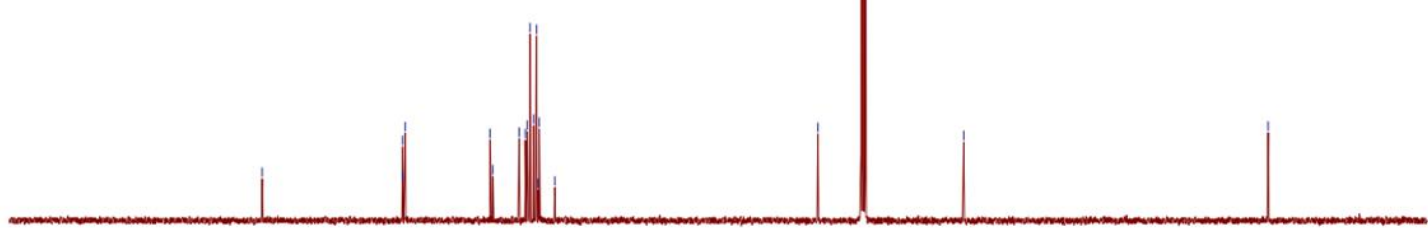


${ }^{1} \mathrm{H}$ NMR of $4 \mathbf{e}\left(400 \mathrm{MHz}, \mathrm{CDCl}_{3}\right)$

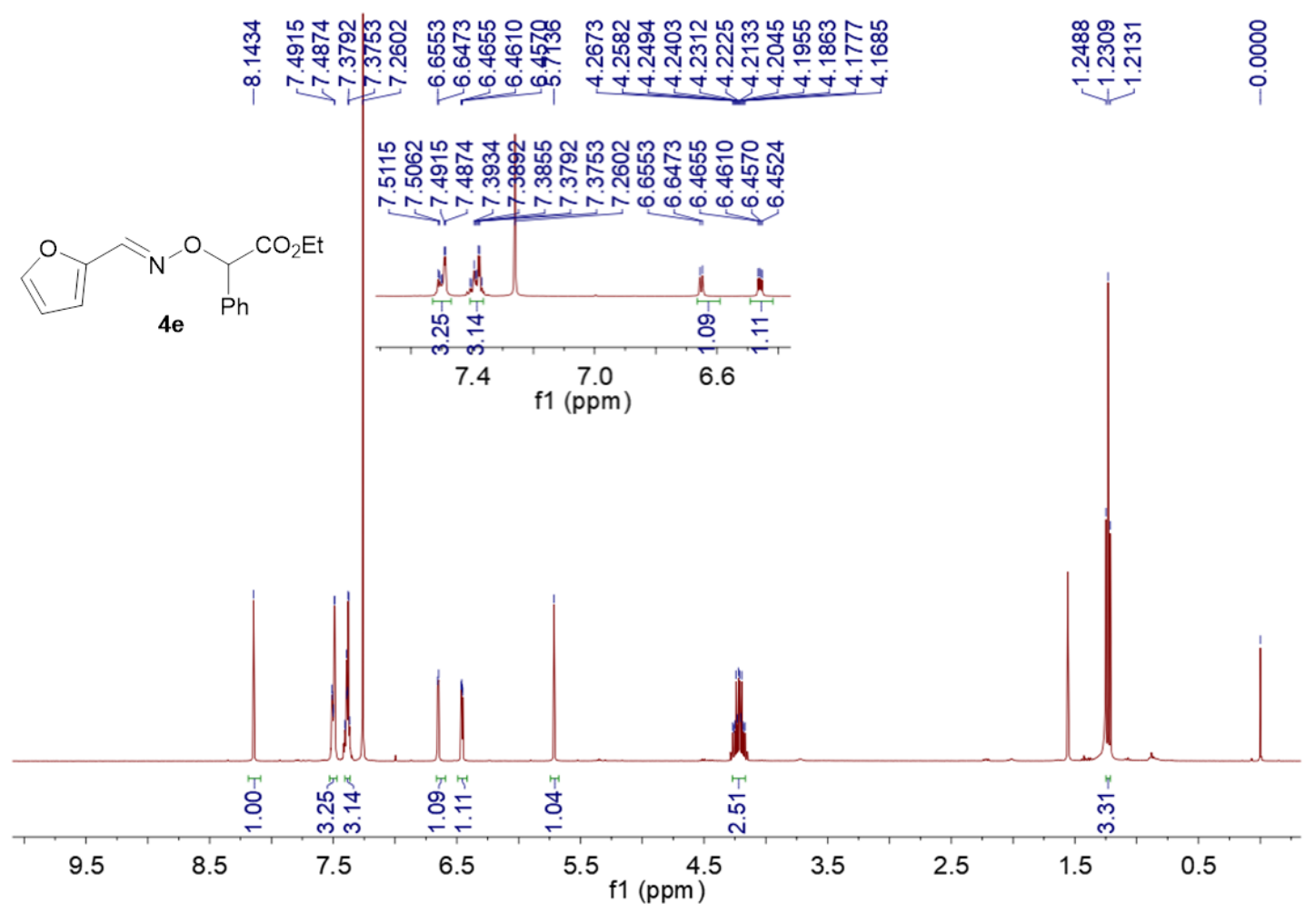

${ }^{13} \mathrm{C}$ NMR of $4 \mathbf{e}\left(100 \mathrm{MHz}, \mathrm{CDCl}_{3}\right)$
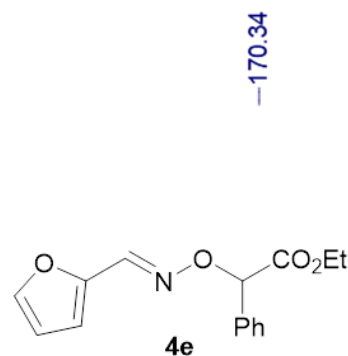

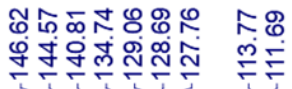

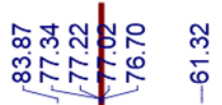

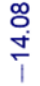

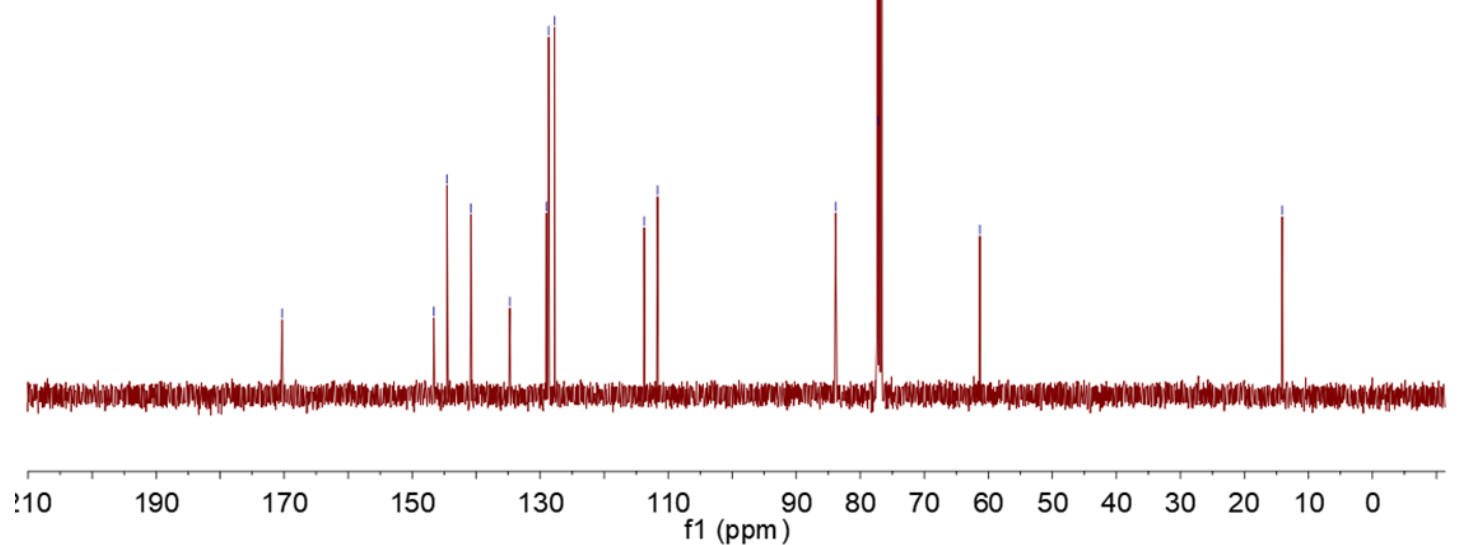


${ }^{1} \mathrm{H}$ NMR of $\mathbf{4 f}\left(400 \mathrm{MHz}, \mathrm{CDCl}_{3}\right)$

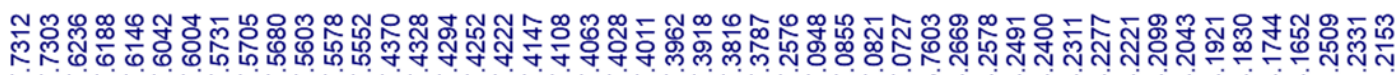

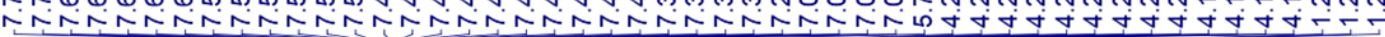
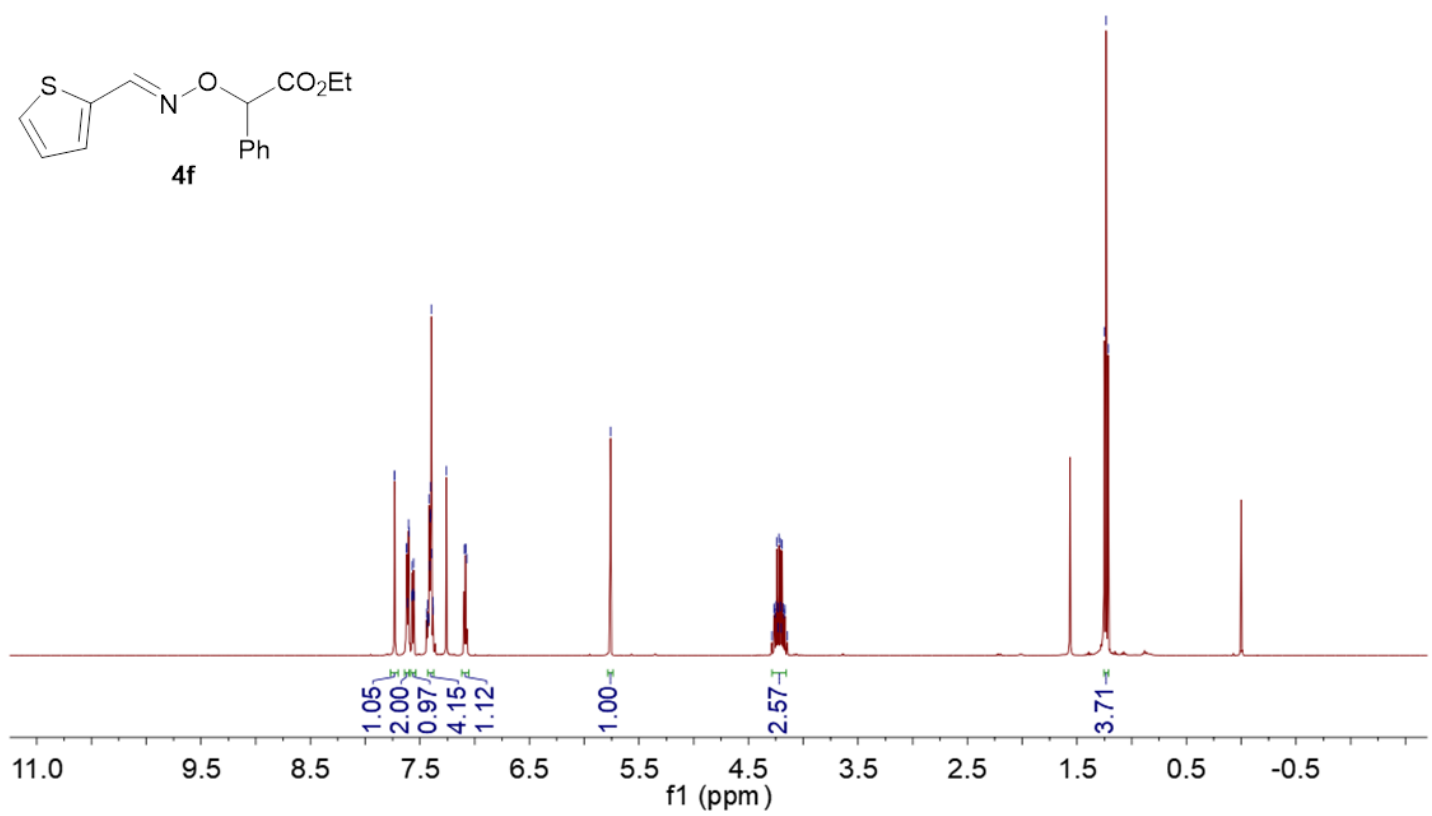

${ }^{13} \mathrm{C}$ NMR of $\mathbf{4 f}\left(100 \mathrm{MHz}, \mathrm{CDCl}_{3}\right)$

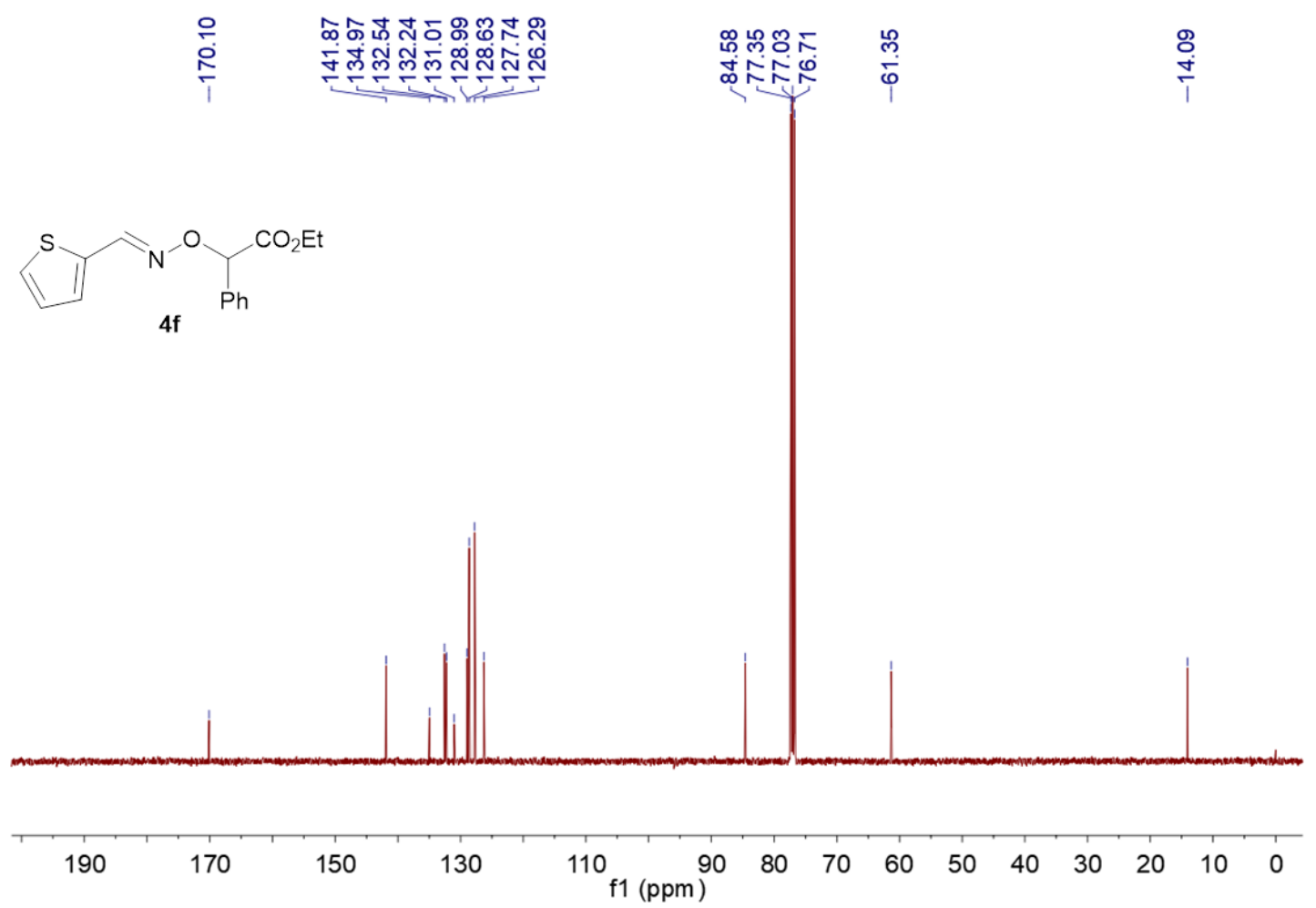


${ }^{1} \mathrm{H}$ NMR of $\mathbf{4 g}\left(400 \mathrm{MHz}, \mathrm{CDCl}_{3}\right)$

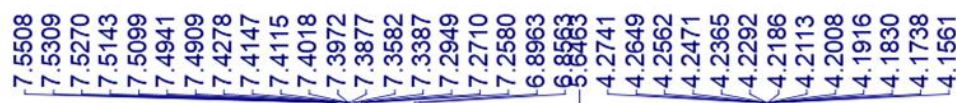

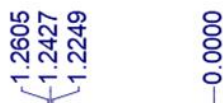
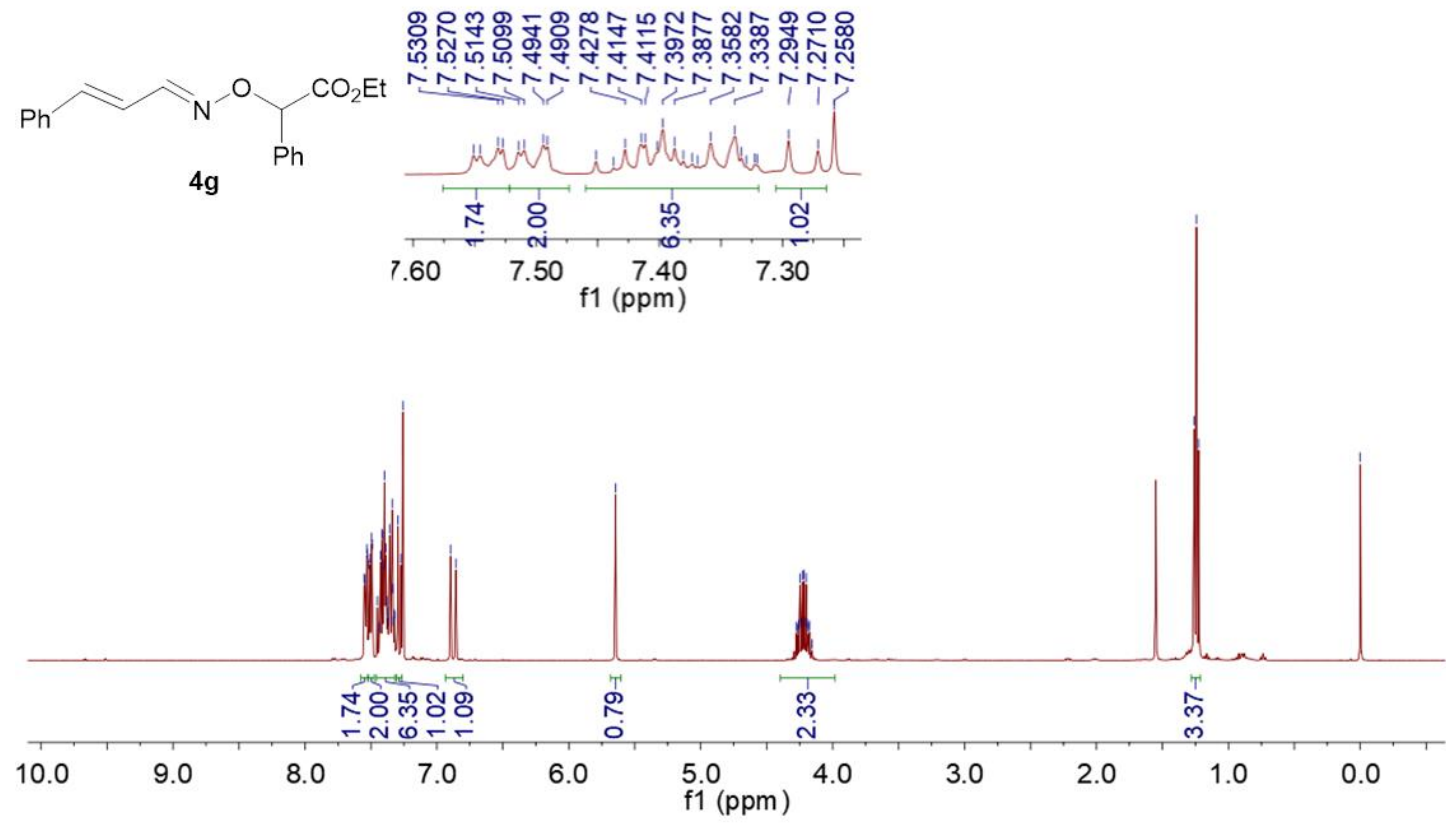

${ }^{13} \mathrm{C}$ NMR of $4 \mathbf{g}\left(100 \mathrm{MHz}, \mathrm{CDCl}_{3}\right)$

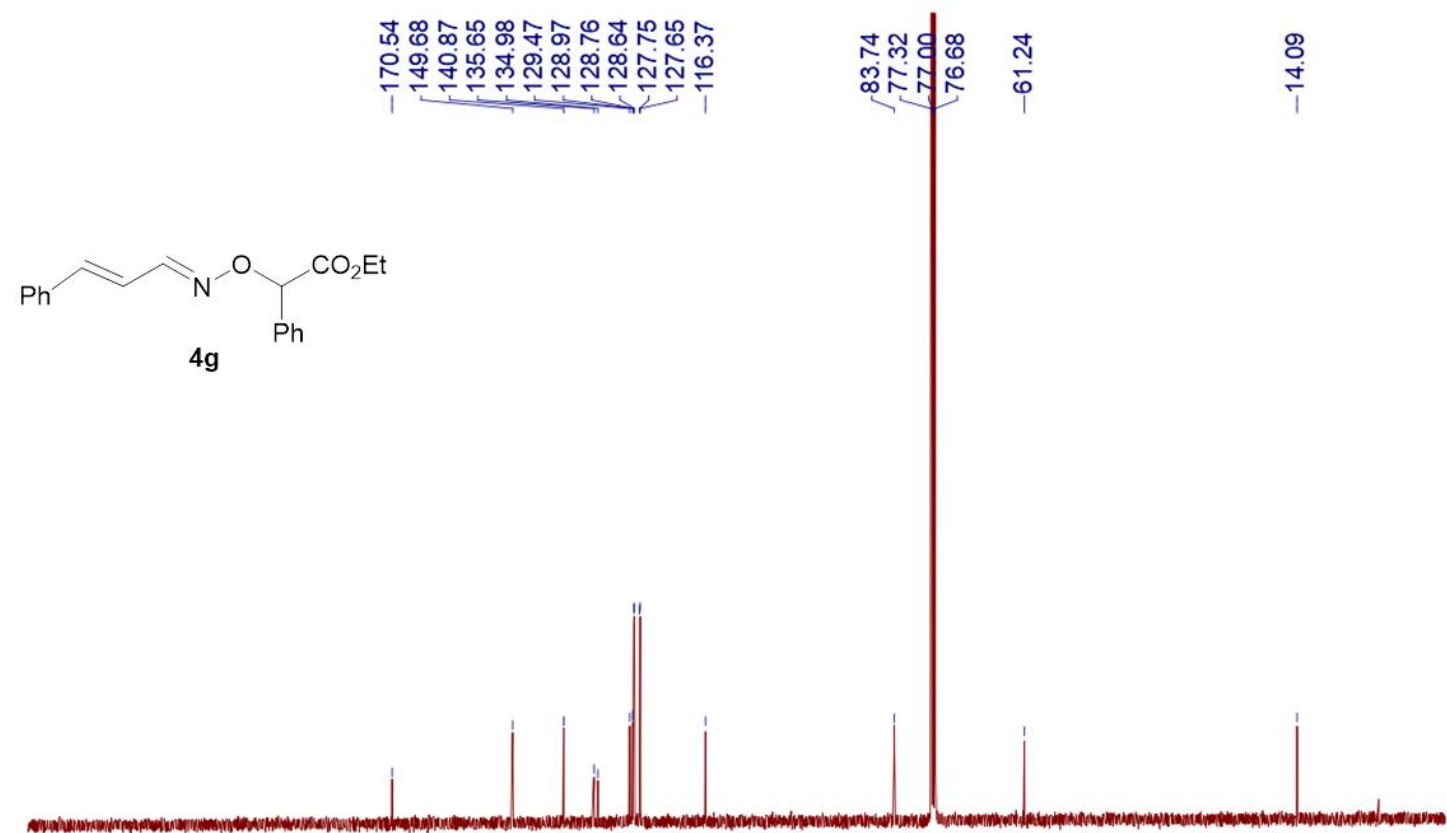

220

200

180

160

140

$120(\mathrm{fpm})$

80

60

$40 \quad 20 \quad 0$ 0 
${ }^{1} \mathrm{H}$ NMR of $\mathbf{4 h}\left(400 \mathrm{MHz}, \mathrm{CDCl}_{3}\right)$

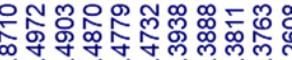

$\mathrm{CH}_{3} \mathrm{~N}_{\mathrm{Ph}}^{-} \mathrm{CO}_{2} \mathrm{Et}$

$4 \mathrm{~h}$

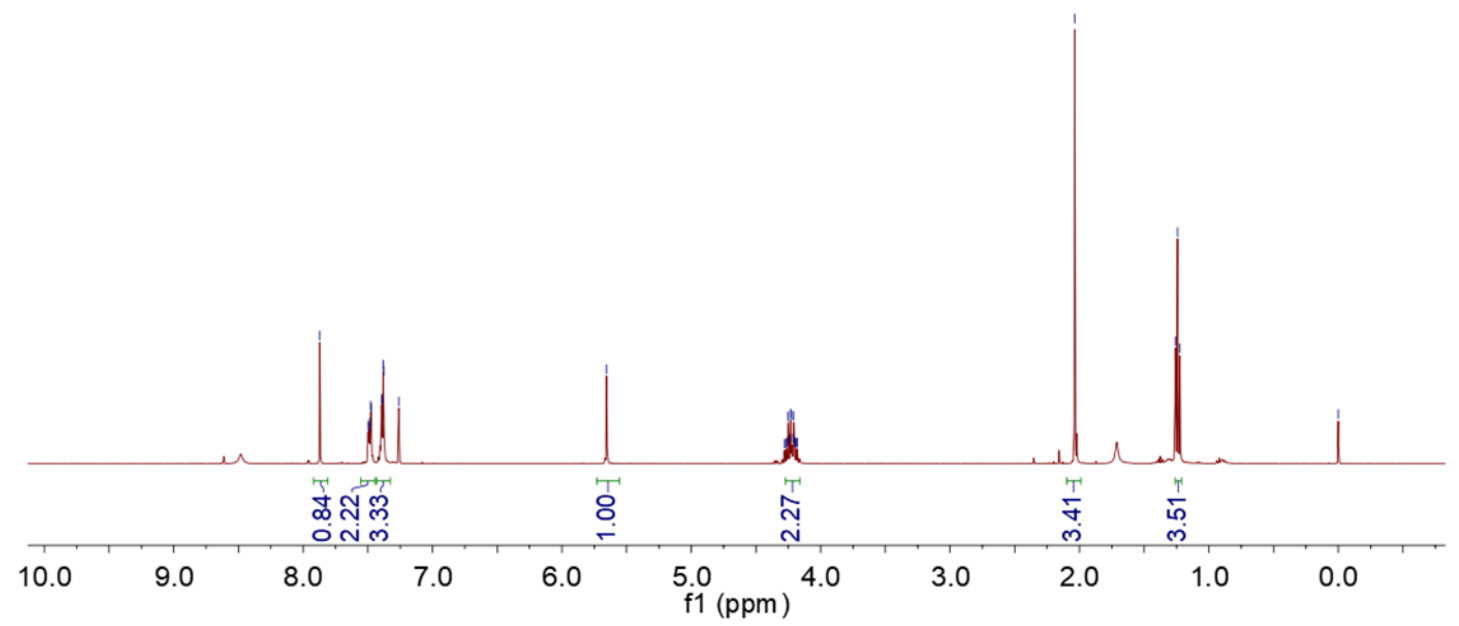

${ }^{13} \mathrm{C}$ NMR of $4 \mathbf{h}\left(100 \mathrm{MHz}, \mathrm{CDCl}_{3}\right)$

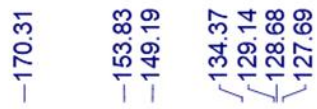

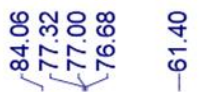

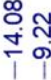

$\mathrm{CH}_{3} \mathrm{~N}^{-} \mathrm{Ph}^{\mathrm{O}} \mathrm{CO}_{2} \mathrm{Et}$

$4 \mathrm{~h}$

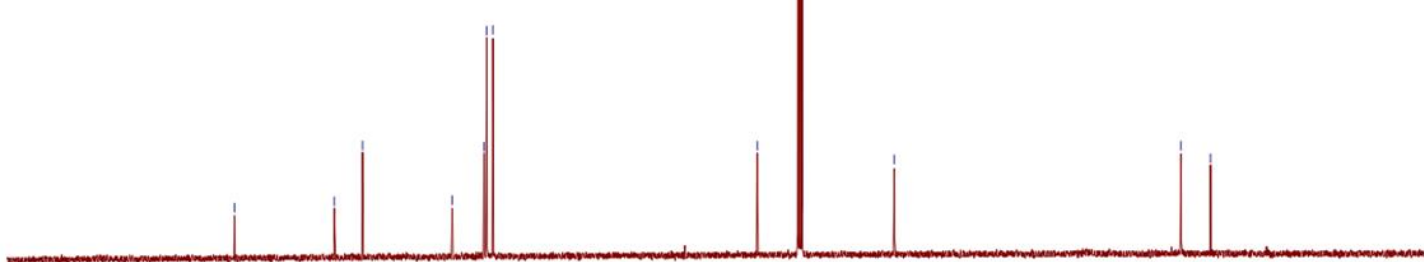

200

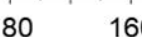

140

120

f1 (ppm)

60

$40 \quad 20$

$0 \quad-10$ 
${ }^{1} \mathrm{H}$ NMR of $4 \mathbf{i}\left(400 \mathrm{MHz}, \mathrm{CDCl}_{3}\right)$

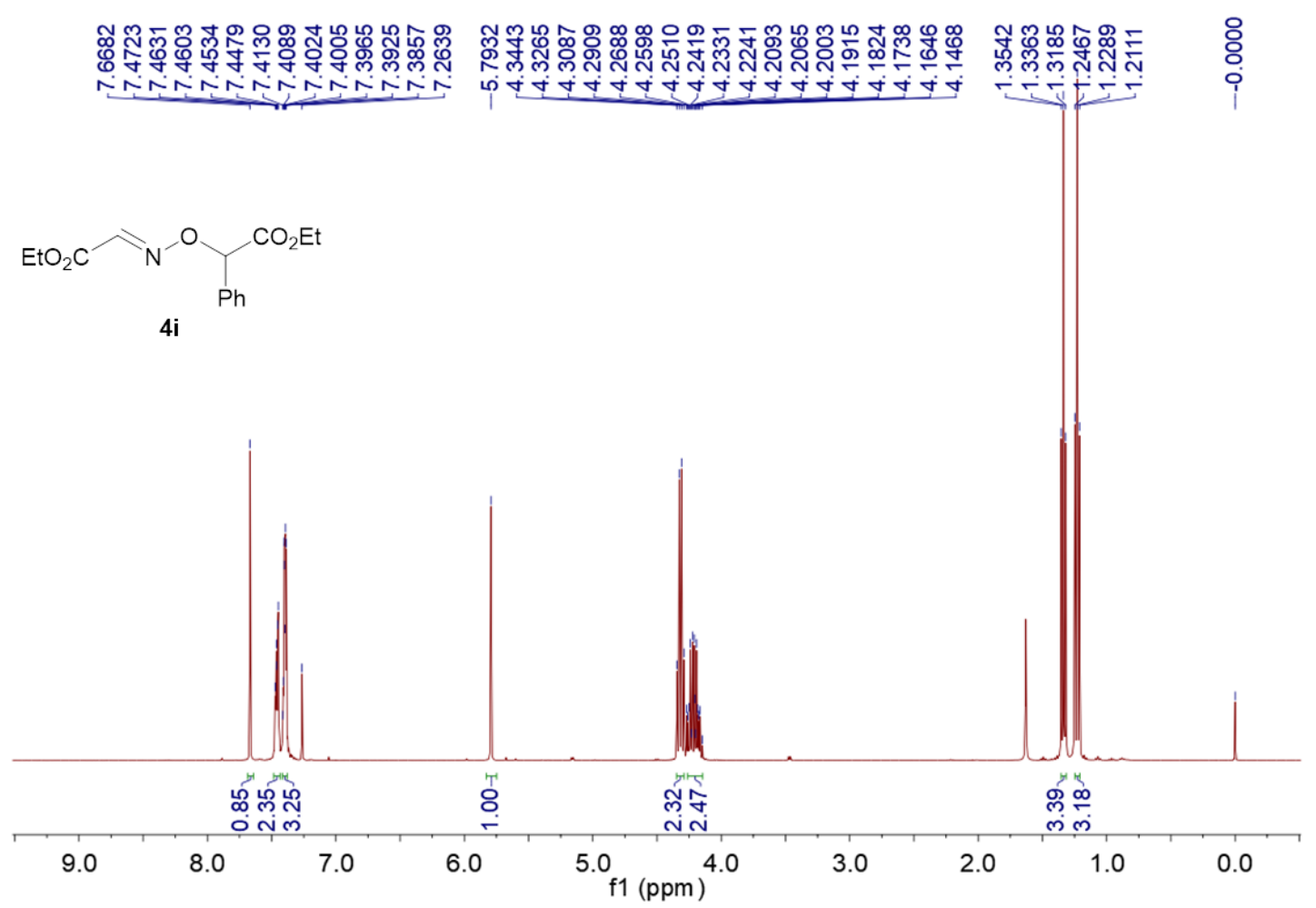

${ }^{13} \mathrm{C} \mathrm{NMR}$ of $4 \mathbf{i}\left(100 \mathrm{MHz}, \mathrm{CDCl}_{3}\right)$

\begin{tabular}{|c|c|c|c|}
\hline 웅 & 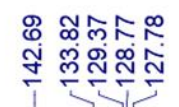 & 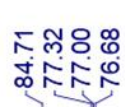 & க் \\
\hline
\end{tabular}

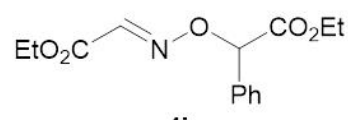

$4 \mathbf{i}$

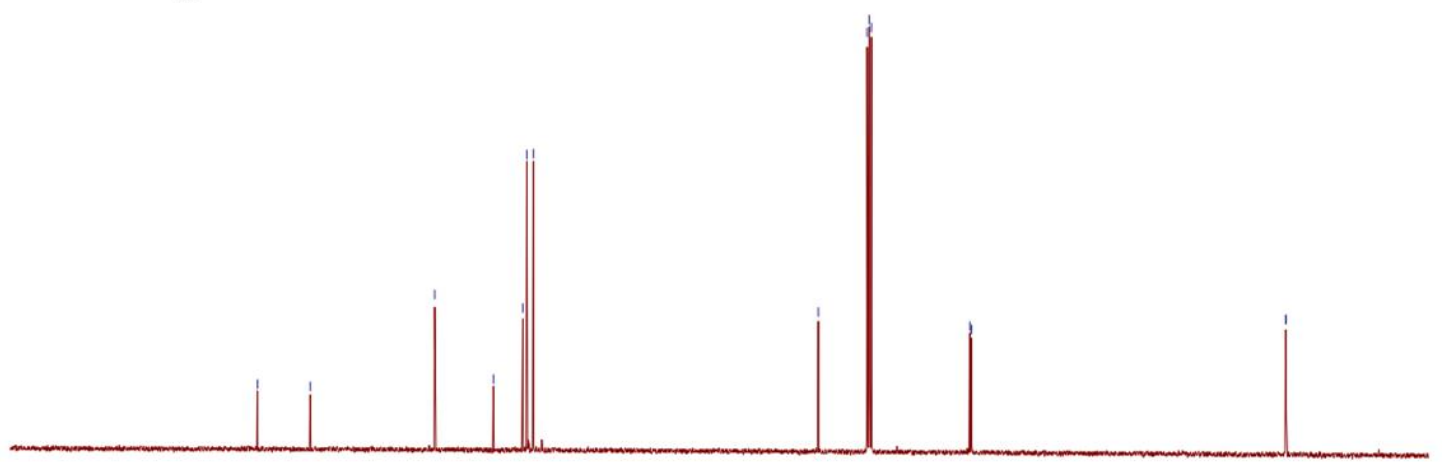


${ }^{1} \mathrm{H}$ NMR of $\mathbf{4 j}\left(400 \mathrm{MHz}, \mathrm{CDCl}_{3}\right)$

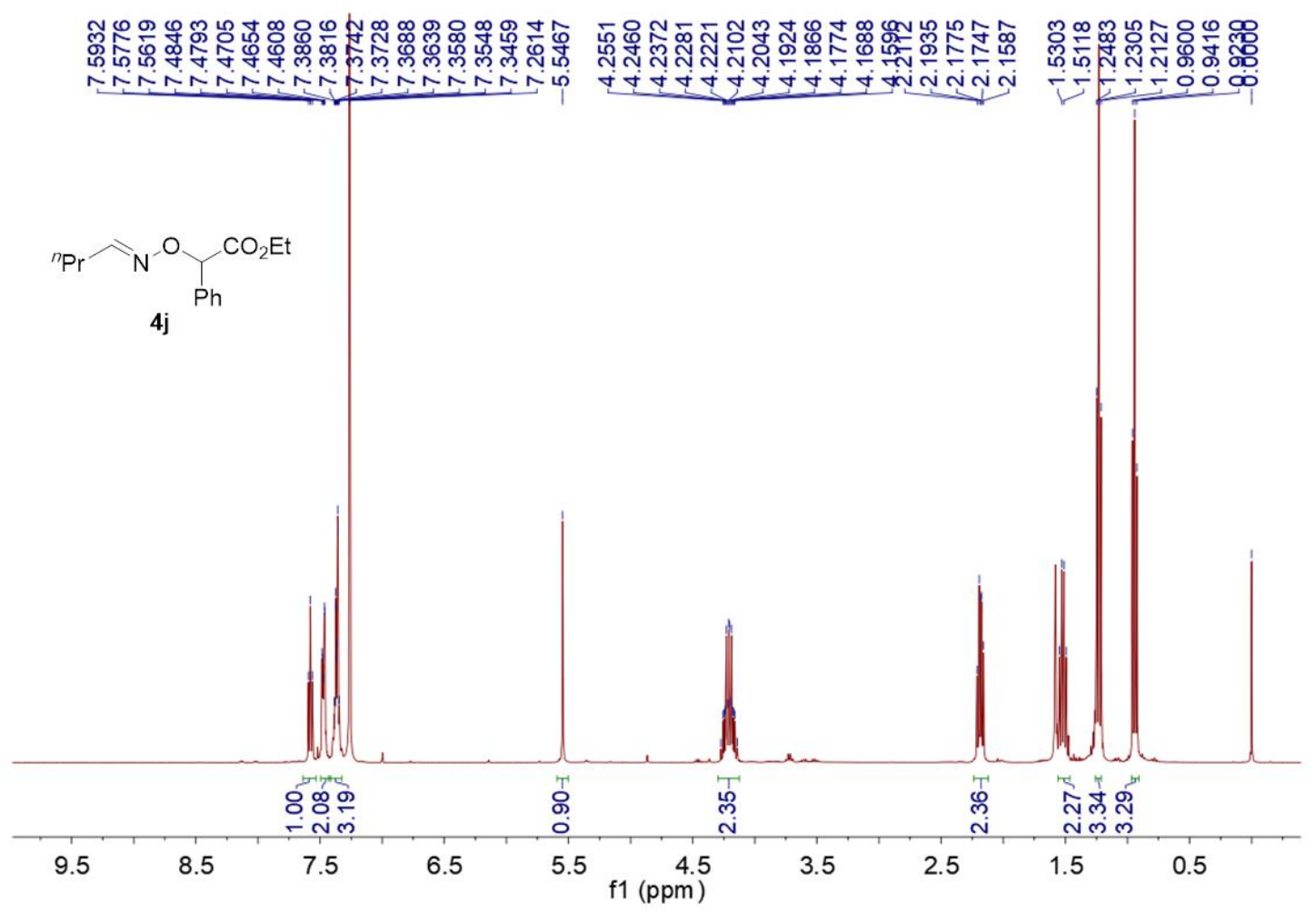

${ }^{13} \mathrm{C}$ NMR of $4 \mathbf{j}\left(100 \mathrm{MHz}, \mathrm{CDCl}_{3}\right)$

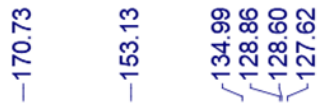

$$
\begin{aligned}
& { }^{n} \mathrm{Pr}{ }_{4 j}{ }_{\mathrm{N}}{ }^{\mathrm{O}} \mathrm{Ph}^{\mathrm{CO}_{2} \mathrm{Et}}
\end{aligned}
$$

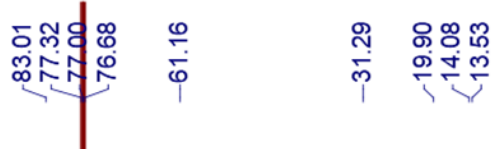

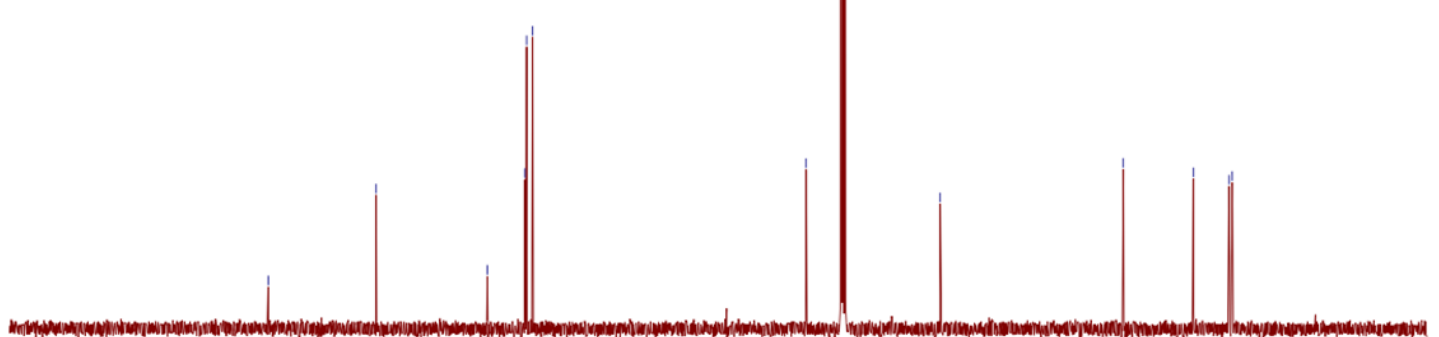

200

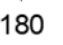

160

140

120

100

80

60

40

20

0 
${ }^{1} \mathrm{H}$ NMR of $4 \mathbf{k}\left(400 \mathrm{MHz}, \mathrm{CDCl}_{3}\right)$
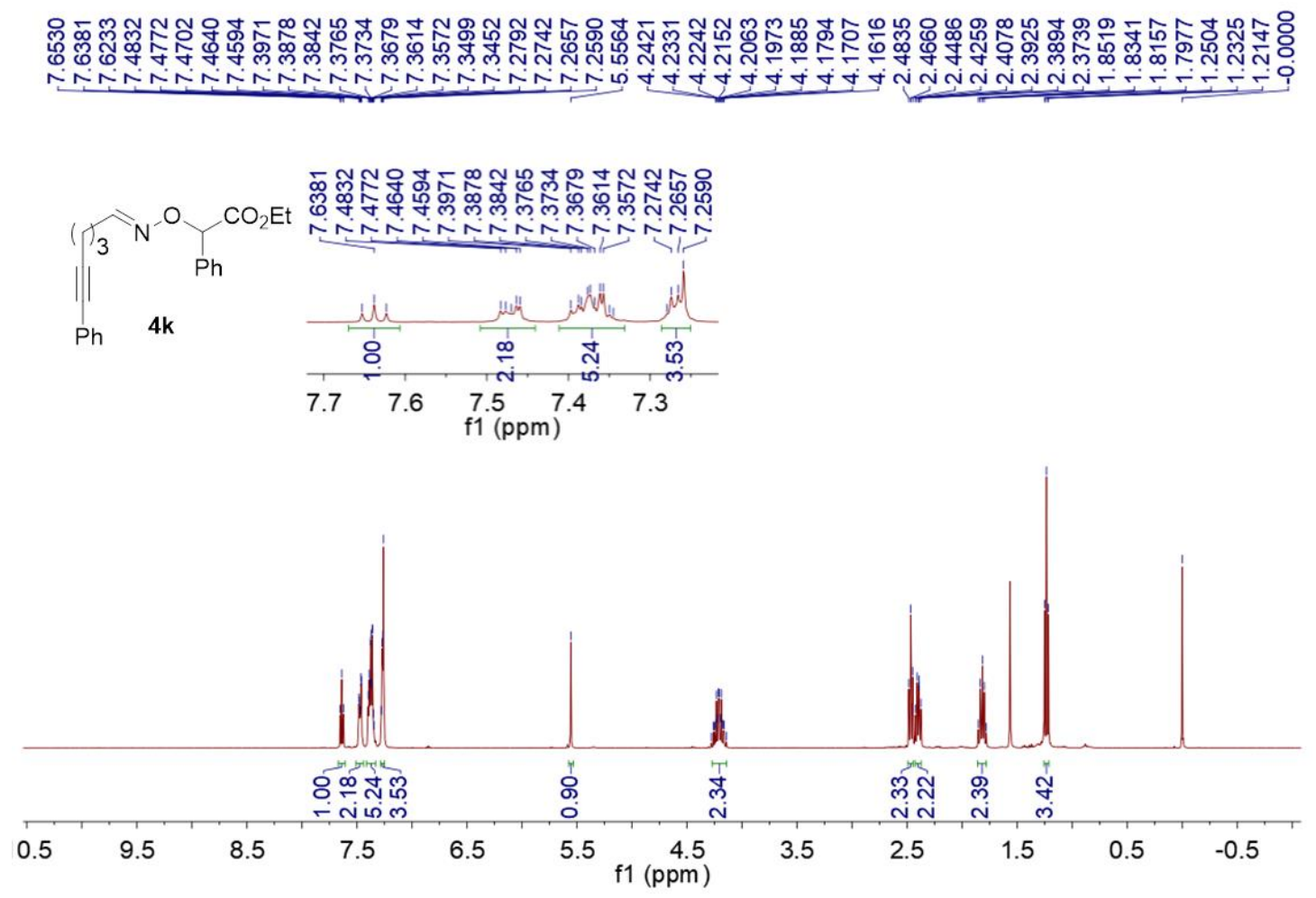

${ }^{13} \mathrm{C}$ NMR of $4 \mathbf{k}\left(100 \mathrm{MHz}, \mathrm{CDCl}_{3}\right)$

$$
\left.\right|_{\mathrm{Ph}} 4 \mathbf{k}
$$

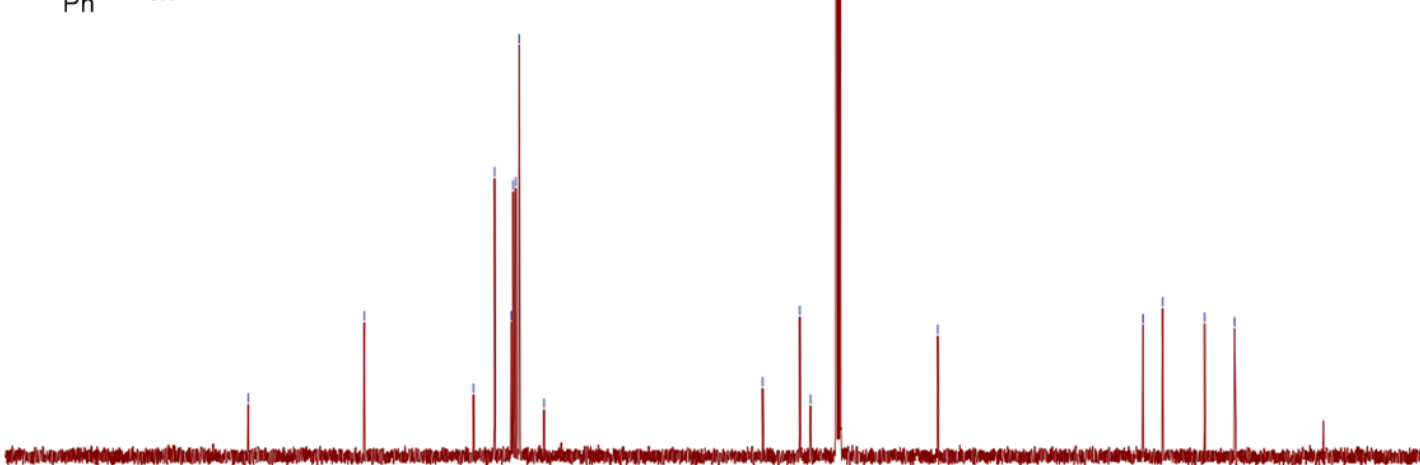

$\begin{array}{lllll}190 & 170 & 150 & 130 & 110 \quad \begin{array}{r}90 \\ \mathrm{f} 1(\mathrm{ppm})\end{array}\end{array}$

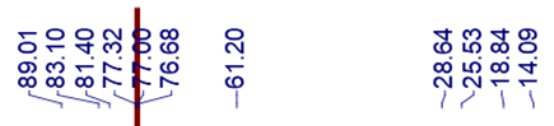


${ }^{1} \mathrm{H}$ NMR of $4 \mathbf{l}\left(400 \mathrm{MHz}, \mathrm{CDCl}_{3}\right)$

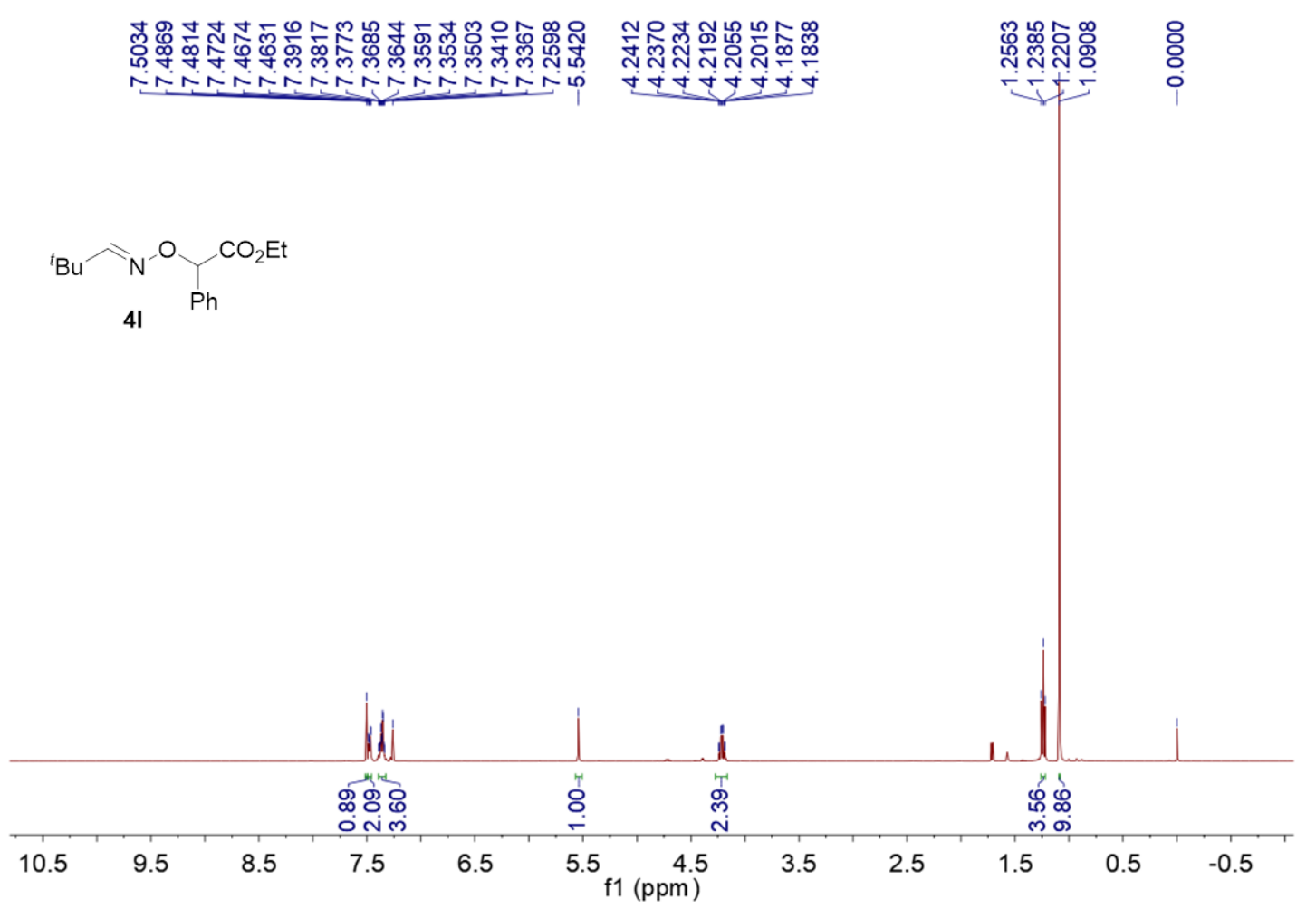

${ }^{13} \mathrm{C}$ NMR of $4 \mathbf{l}\left(100 \mathrm{MHz}, \mathrm{CDCl}_{3}\right)$

\begin{tabular}{|c|c|c|c|c|}
\hline 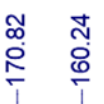 & 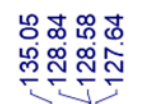 & 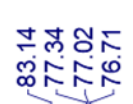 & $\stackrel{8}{\dot{\varphi}}$ & 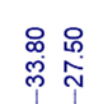 \\
\hline
\end{tabular}

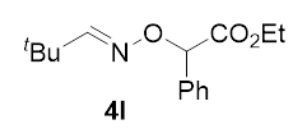

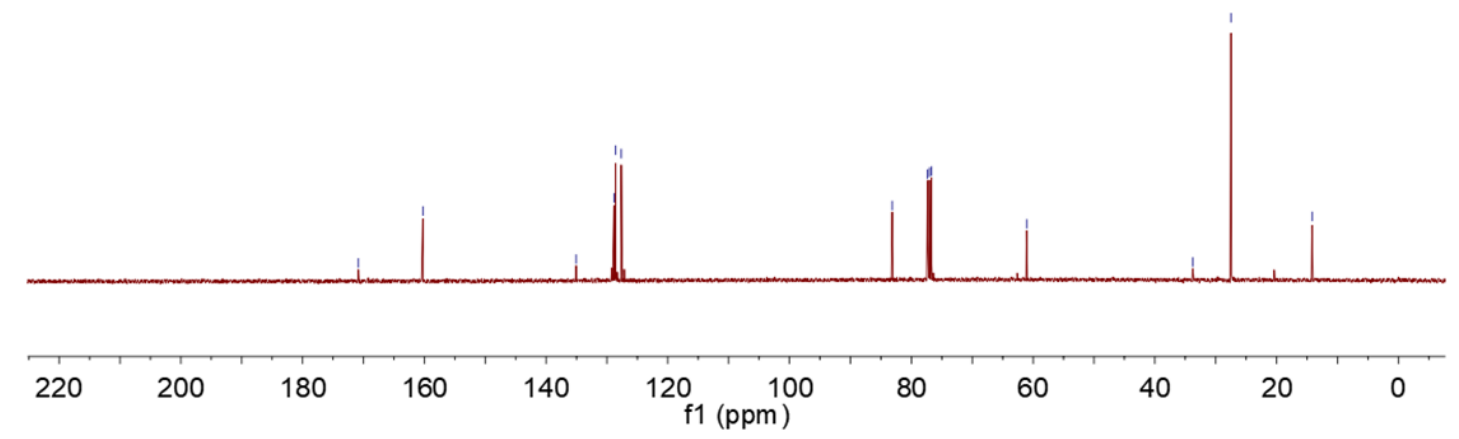


${ }^{1} \mathrm{H}$ NMR of $\mathbf{4 m}\left(400 \mathrm{MHz}, \mathrm{CDCl}_{3}\right)$

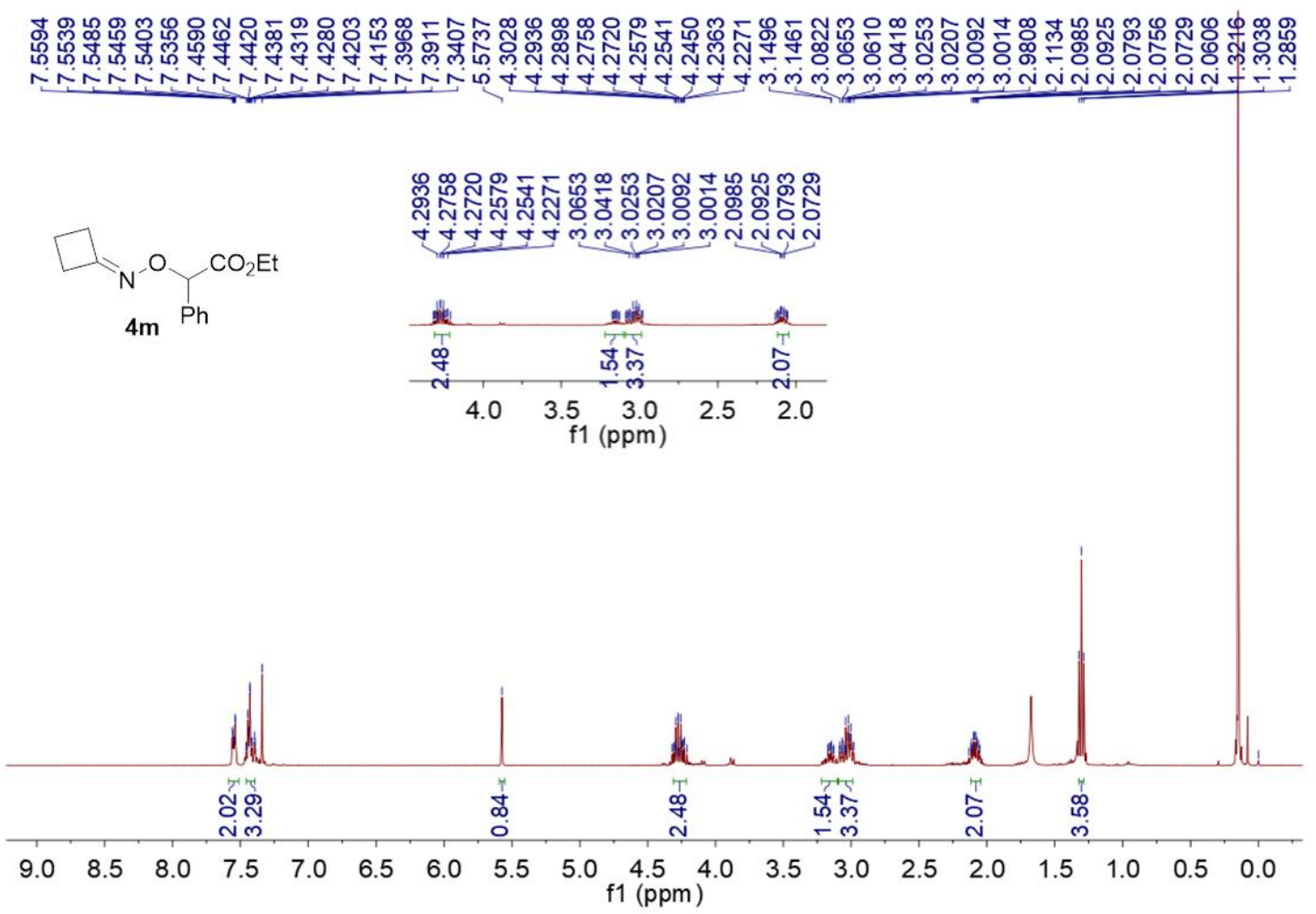

${ }^{13} \mathrm{C} \mathrm{NMR}$ of $4 \mathbf{m}\left(100 \mathrm{MHz}, \mathrm{CDCl}_{3}\right)$

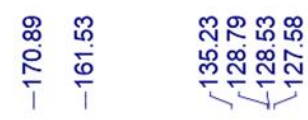

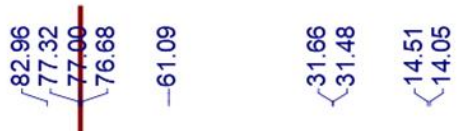

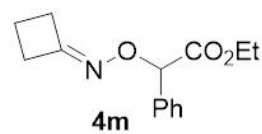

$4 \mathrm{~m} P \mathrm{Ph}$ 
${ }^{1} \mathrm{H}$ NMR of $4 \mathbf{n}\left(400 \mathrm{MHz}\right.$, DMSO- $\left.d_{6}\right)$

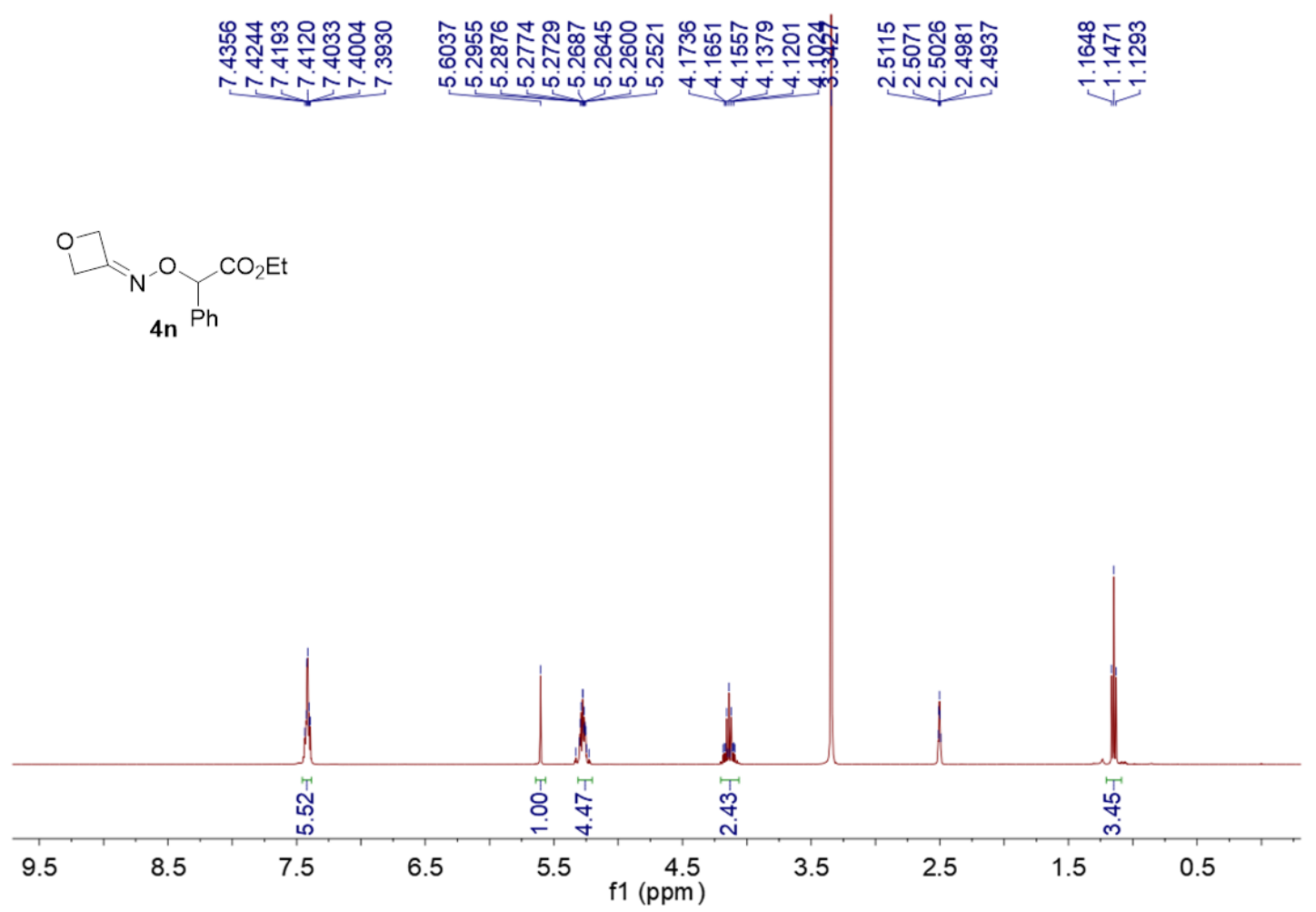

${ }^{13} \mathrm{C}$ NMR of $4 \mathbf{n}\left(100 \mathrm{MHz}\right.$, DMSO- $\left.d_{6}\right)$

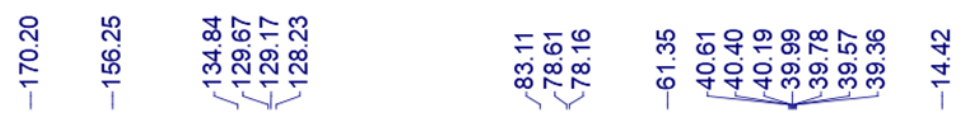
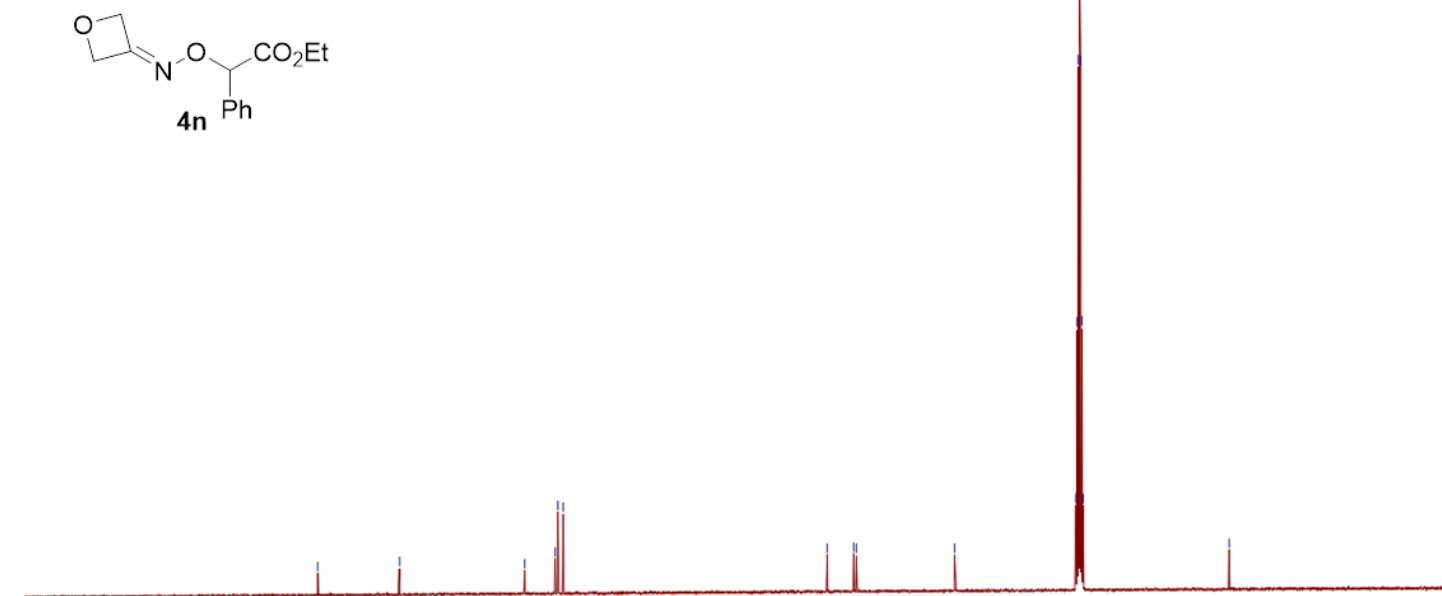

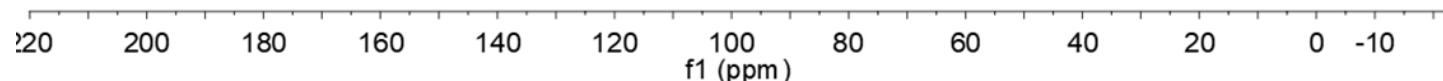


${ }^{1} \mathrm{H}$ NMR of $40\left(400 \mathrm{MHz}, \mathrm{CDCl}_{3}\right)$

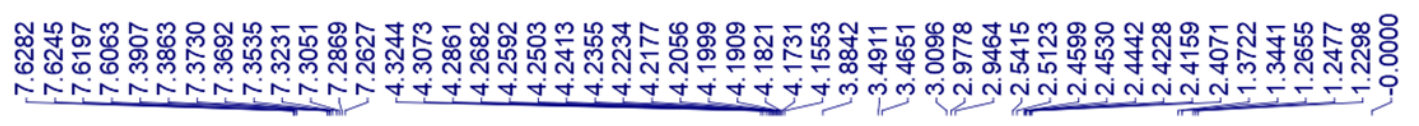

$\underbrace{\mathrm{BocN}}_{40} \mathrm{Ph}_{\mathrm{Ph}} \mathrm{CO}_{2} \mathrm{Et}$

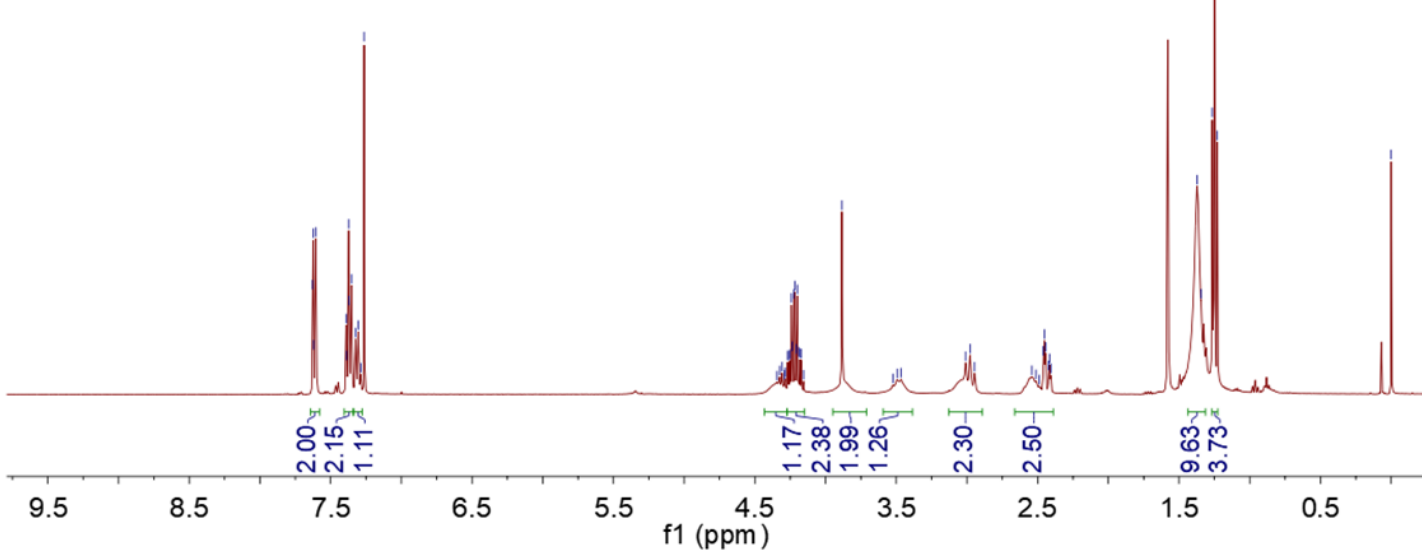

${ }^{13} \mathrm{C}$ NMR of $40\left(100 \mathrm{MHz}, \mathrm{CDCl}_{3}\right)$

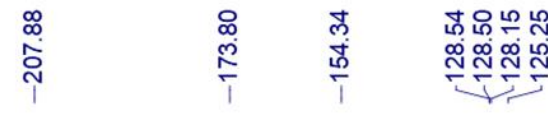

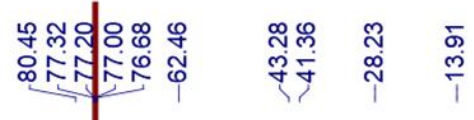<smiles>CCOC(=O)C(ON=C1CCN(C(=O)OC(C)(C)C)CC1)c1ccccc1</smiles>

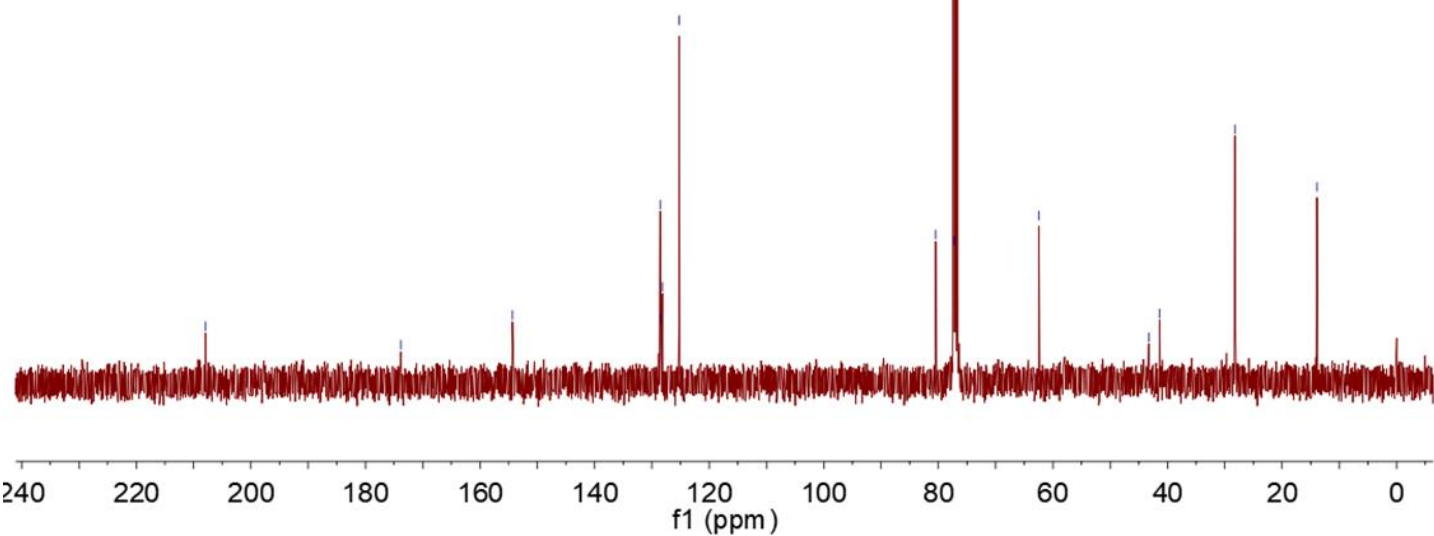


${ }^{1} \mathrm{H}$ NMR of $4 \mathbf{p}\left(400 \mathrm{MHz}, \mathrm{CDCl}_{3}\right)$

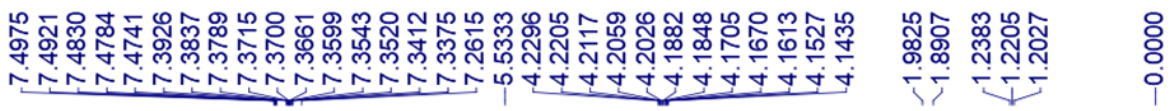

$\mathrm{Me} \overbrace{4 \mathrm{p}}^{\mathrm{Me}} \mathrm{Ph}_{\mathrm{N}}^{-} \mathrm{O}_{2} \mathrm{CO}_{2} \mathrm{Et}$

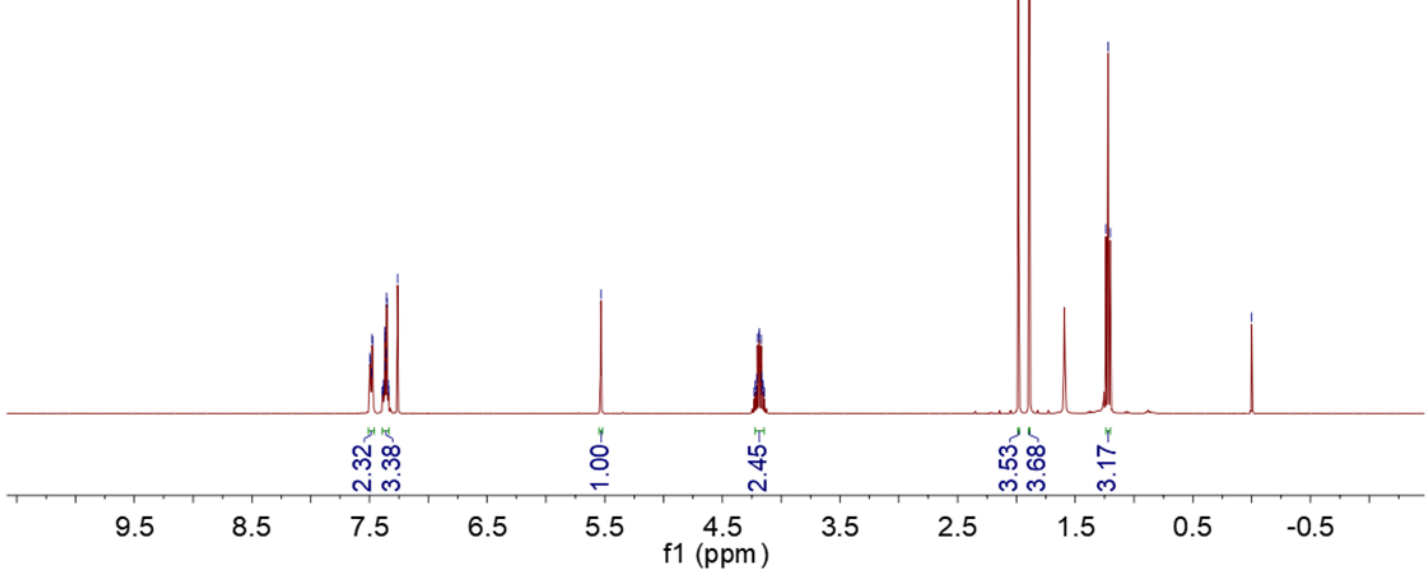

${ }^{13} \mathrm{C}$ NMR of $4 \mathbf{p}\left(100 \mathrm{MHz}, \mathrm{CDCl}_{3}\right)$

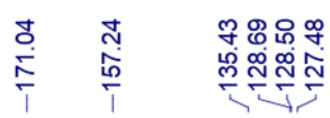

묘융

กั

$\mathrm{Me} \stackrel{\mathrm{N}}{\mathrm{Mp}}_{\mathrm{Ph}}^{\mathrm{O}} \mathrm{CO}_{2} \mathrm{Et}$

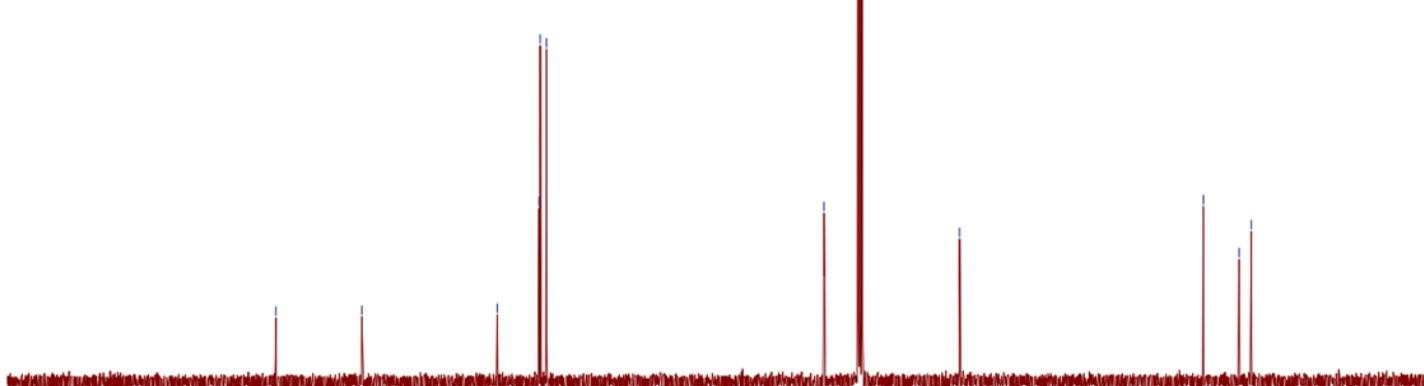

200

180

160

140

120

100

80

60

40

20 
${ }^{1} \mathrm{H}$ NMR of $\mathbf{4 q}\left(400 \mathrm{MHz}, \mathrm{CDCl}_{3}\right)$
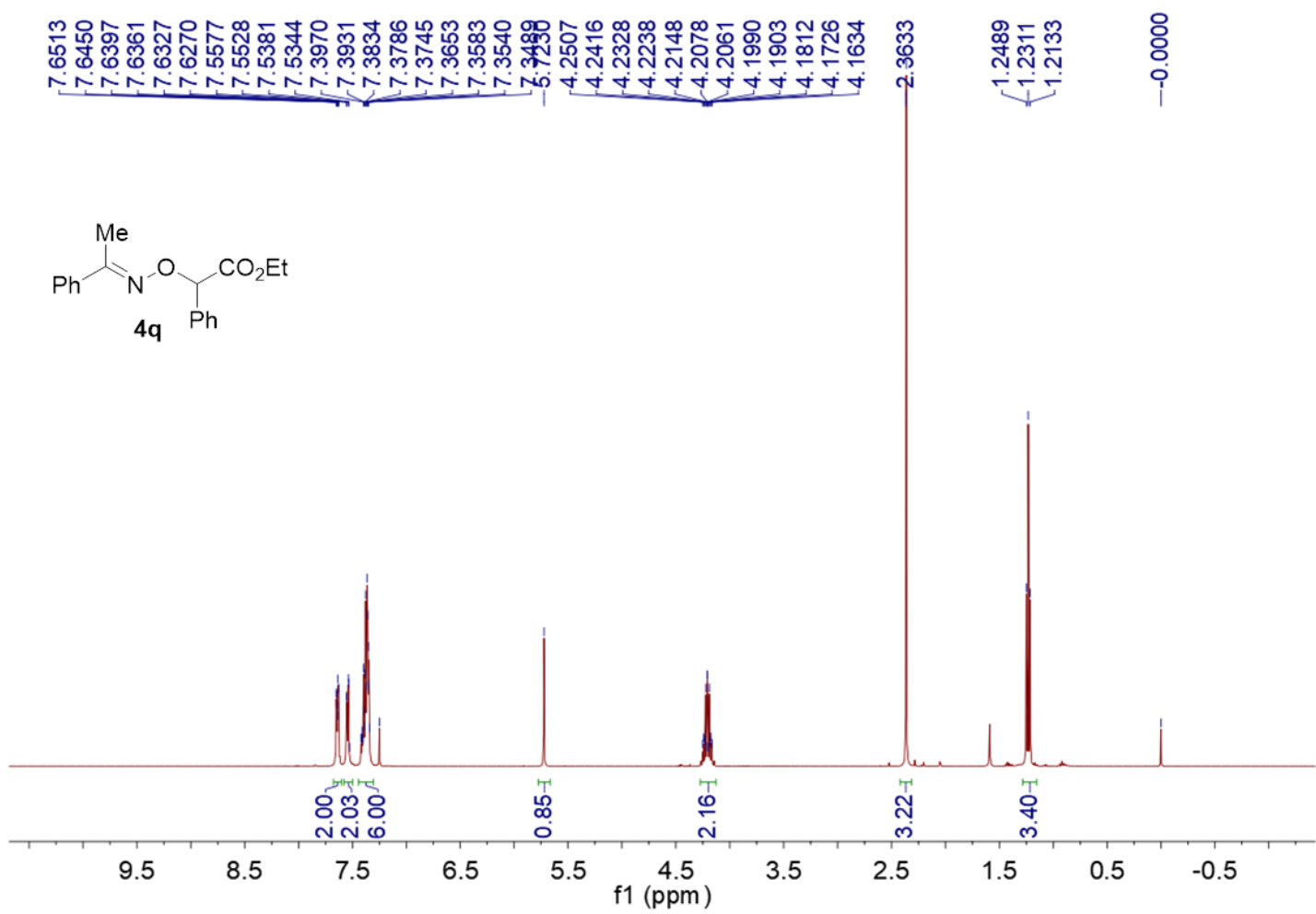

${ }^{13} \mathrm{C}$ NMR of $4 \mathbf{q}\left(100 \mathrm{MHz}, \mathrm{CDCl}_{3}\right)$
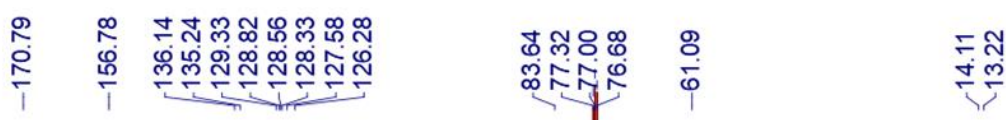

${ }_{\mathrm{Ph}}^{\mathrm{Me}} \overbrace{4 \mathrm{q}}^{\mathrm{Ph}} \mathrm{O}_{Y_{2}}^{\mathrm{CO}_{2} \mathrm{Et}}$

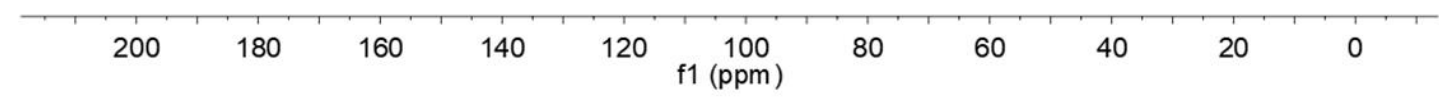


${ }^{1} \mathrm{H}$ NMR of $\mathbf{4 r}\left(400 \mathrm{MHz}, \mathrm{CDCl}_{3}\right)$

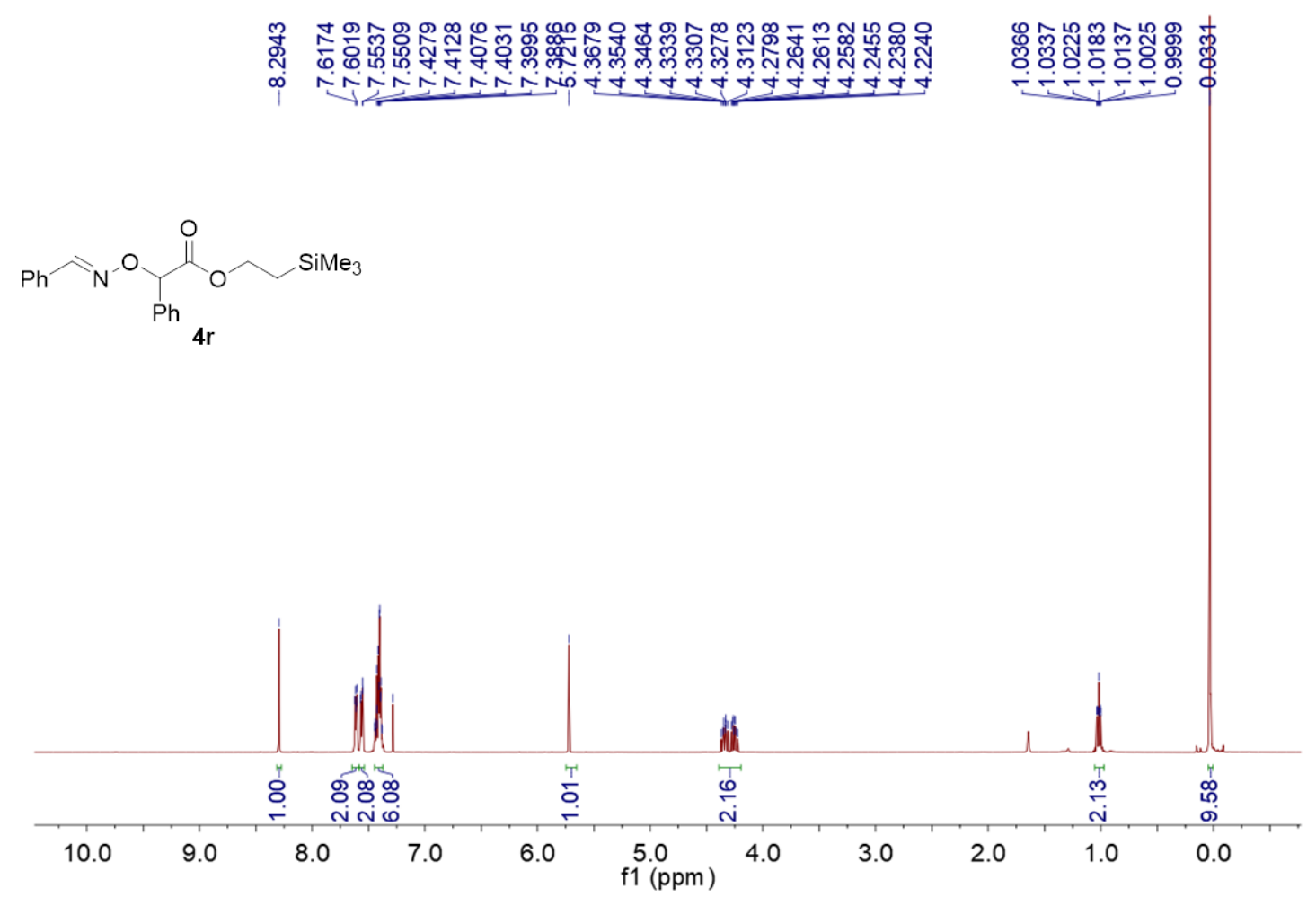

${ }^{13} \mathrm{C}$ NMR of $4 \mathbf{r}\left(100 \mathrm{MHz}, \mathrm{CDCl}_{3}\right)$

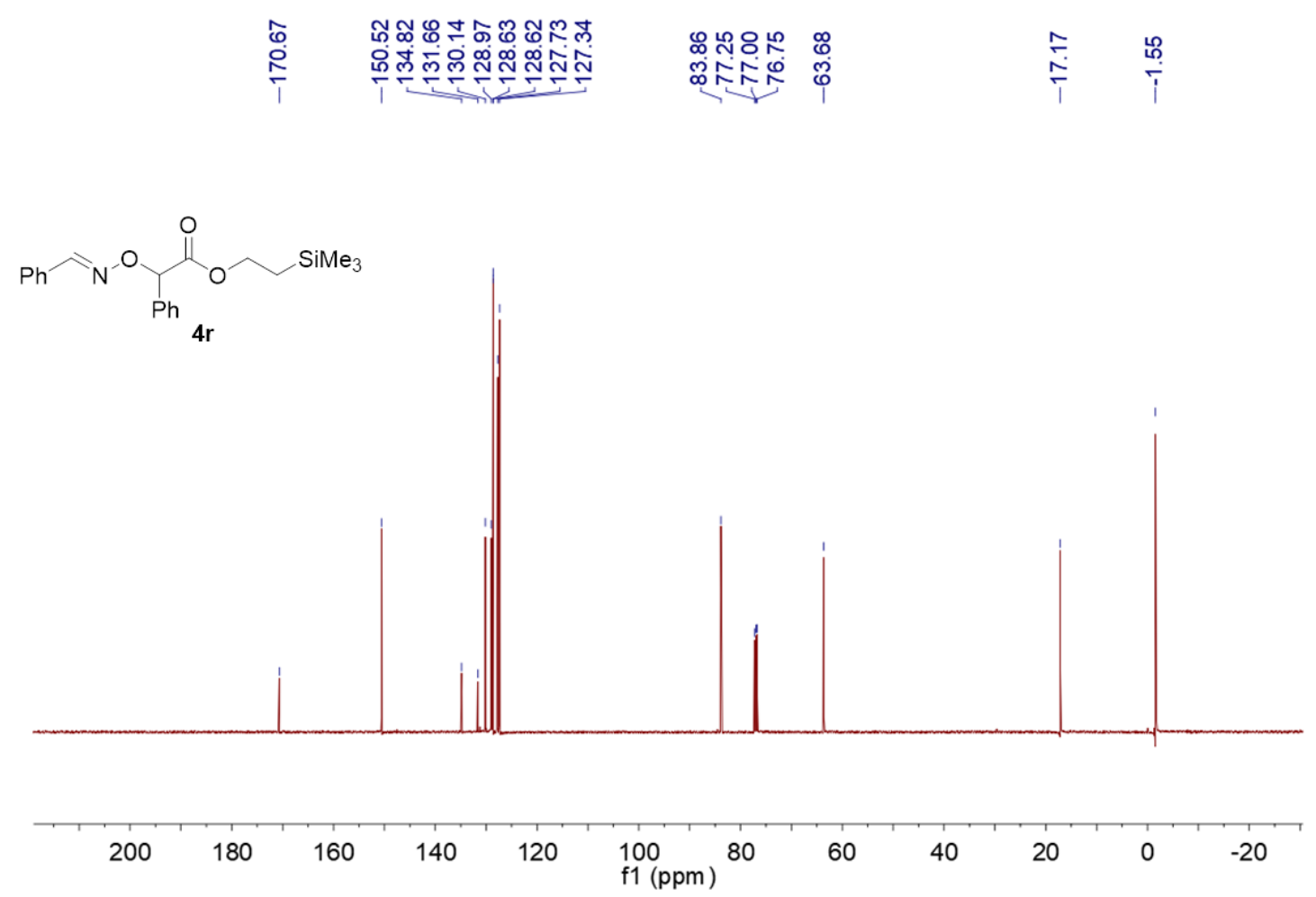


${ }^{1} \mathrm{H}$ NMR of $4 \mathbf{s}\left(400 \mathrm{MHz}, \mathrm{CDCl}_{3}\right)$

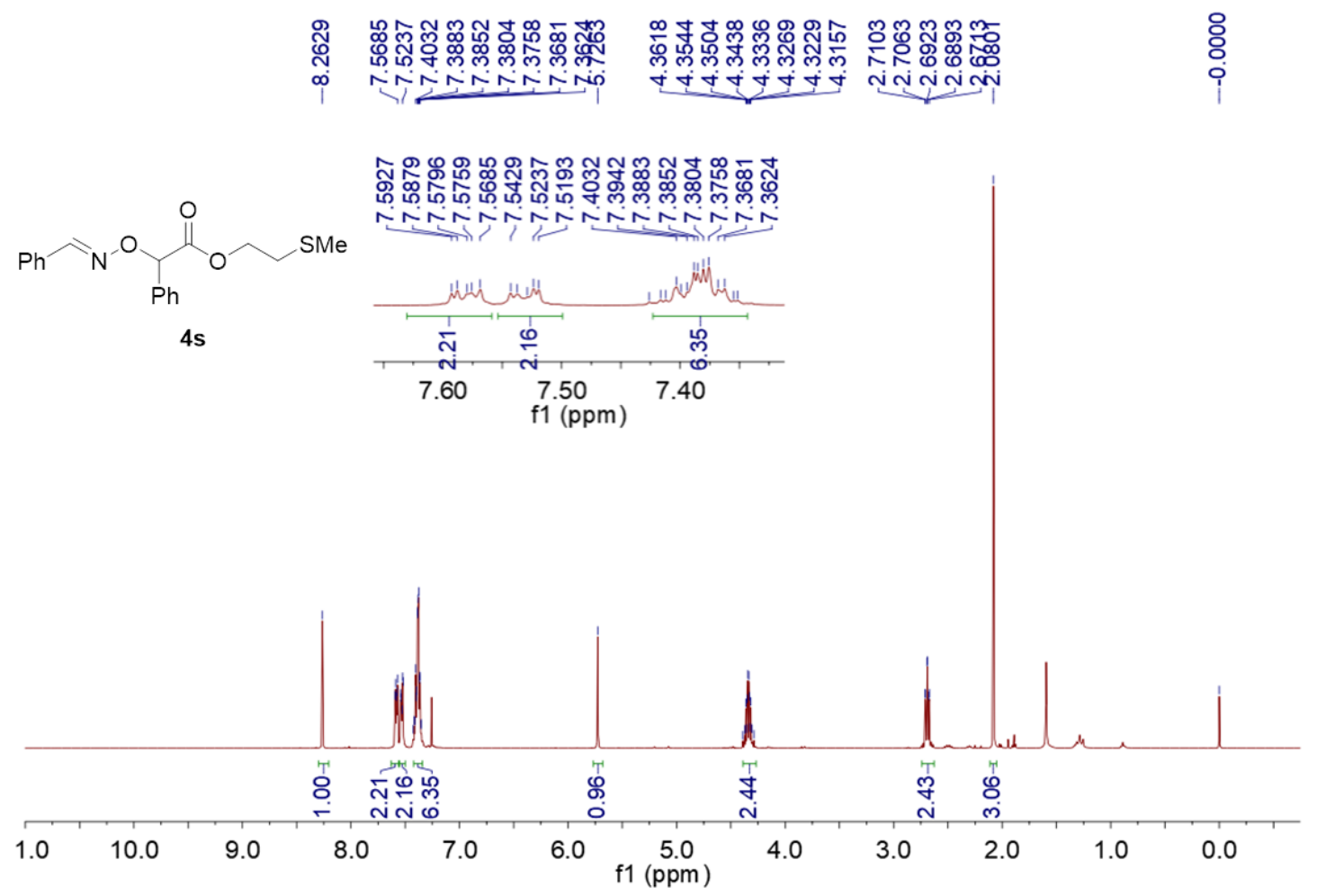

${ }^{13} \mathrm{C} \mathrm{NMR}$ of $4 \mathbf{s}\left(100 \mathrm{MHz}, \mathrm{CDCl}_{3}\right)$

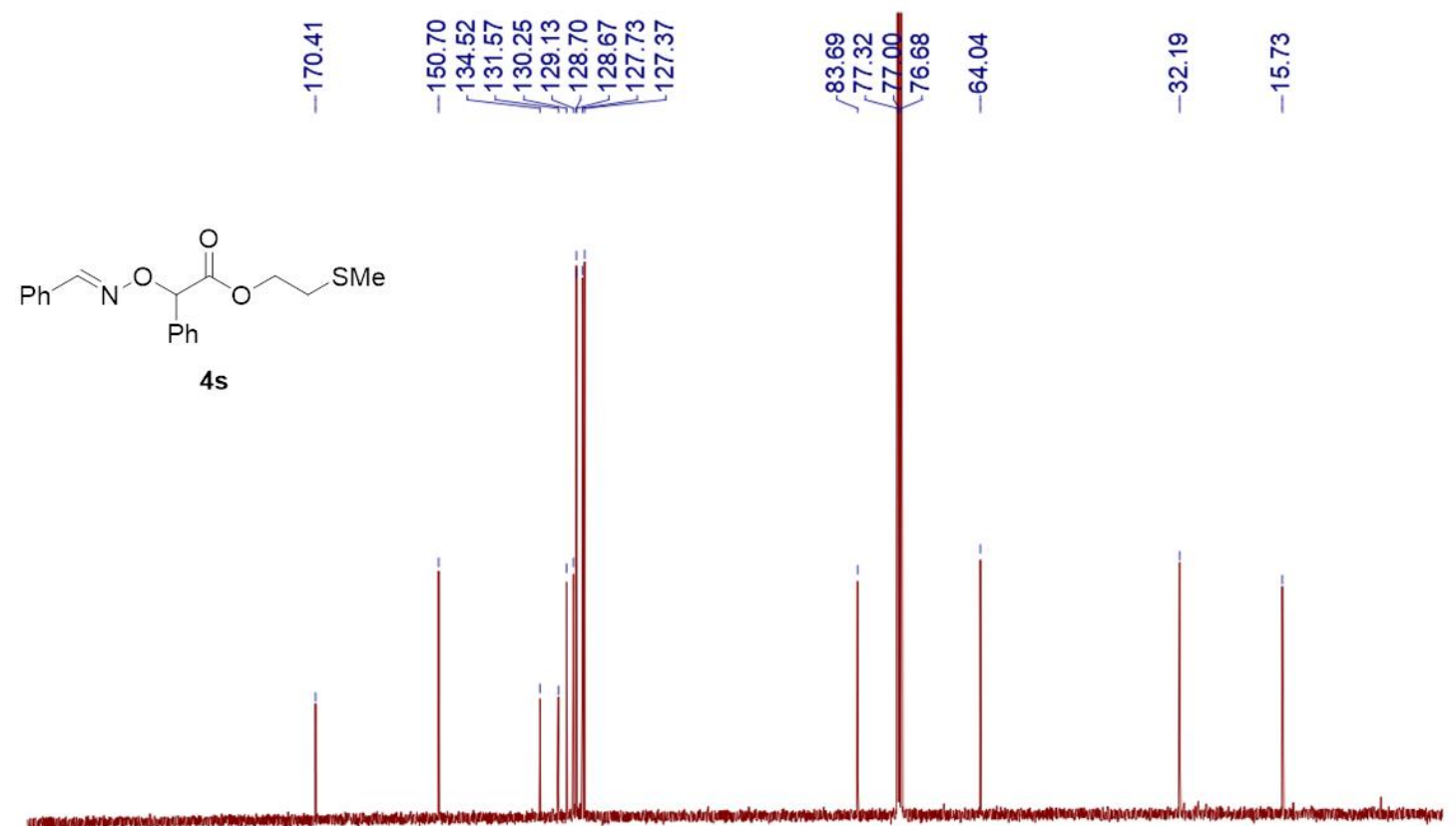

$200180 \quad 160 \quad 140 \quad 120 \begin{gathered}100 \\ \mathrm{f} 1(\mathrm{ppm})\end{gathered}$


${ }^{1} \mathrm{H}$ NMR of $\mathbf{4 t}\left(400 \mathrm{MHz}, \mathrm{CDCl}_{3}\right)$

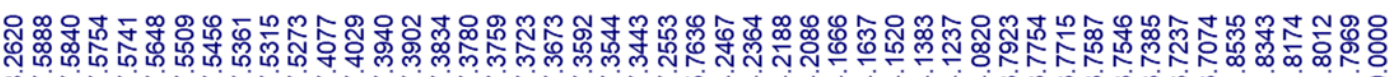

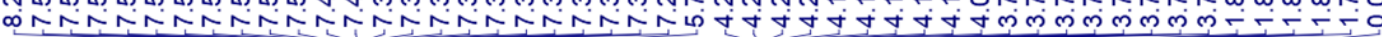

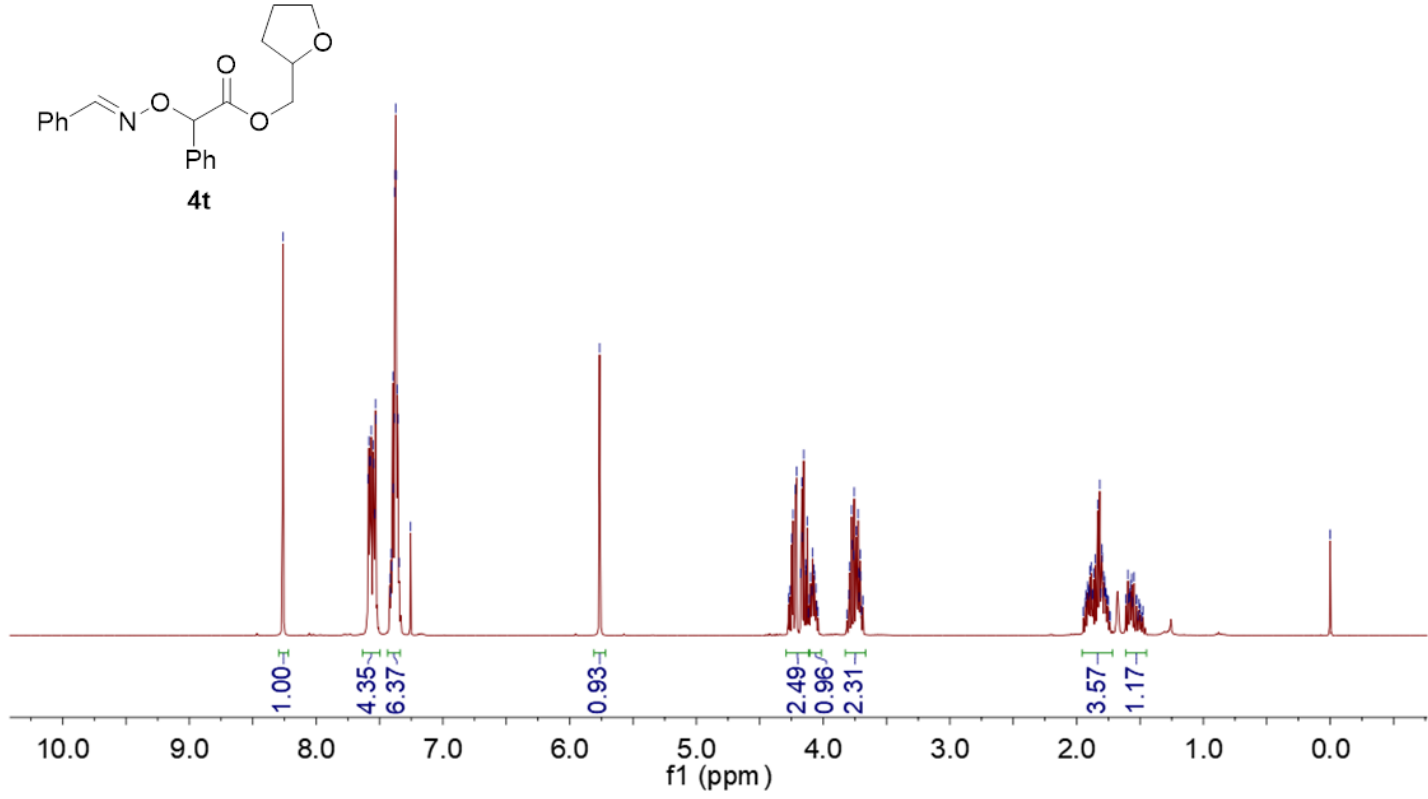

${ }^{13} \mathrm{C}$ NMR of $4 \mathrm{t}\left(100 \mathrm{MHz}, \mathrm{CDCl}_{3}\right)$

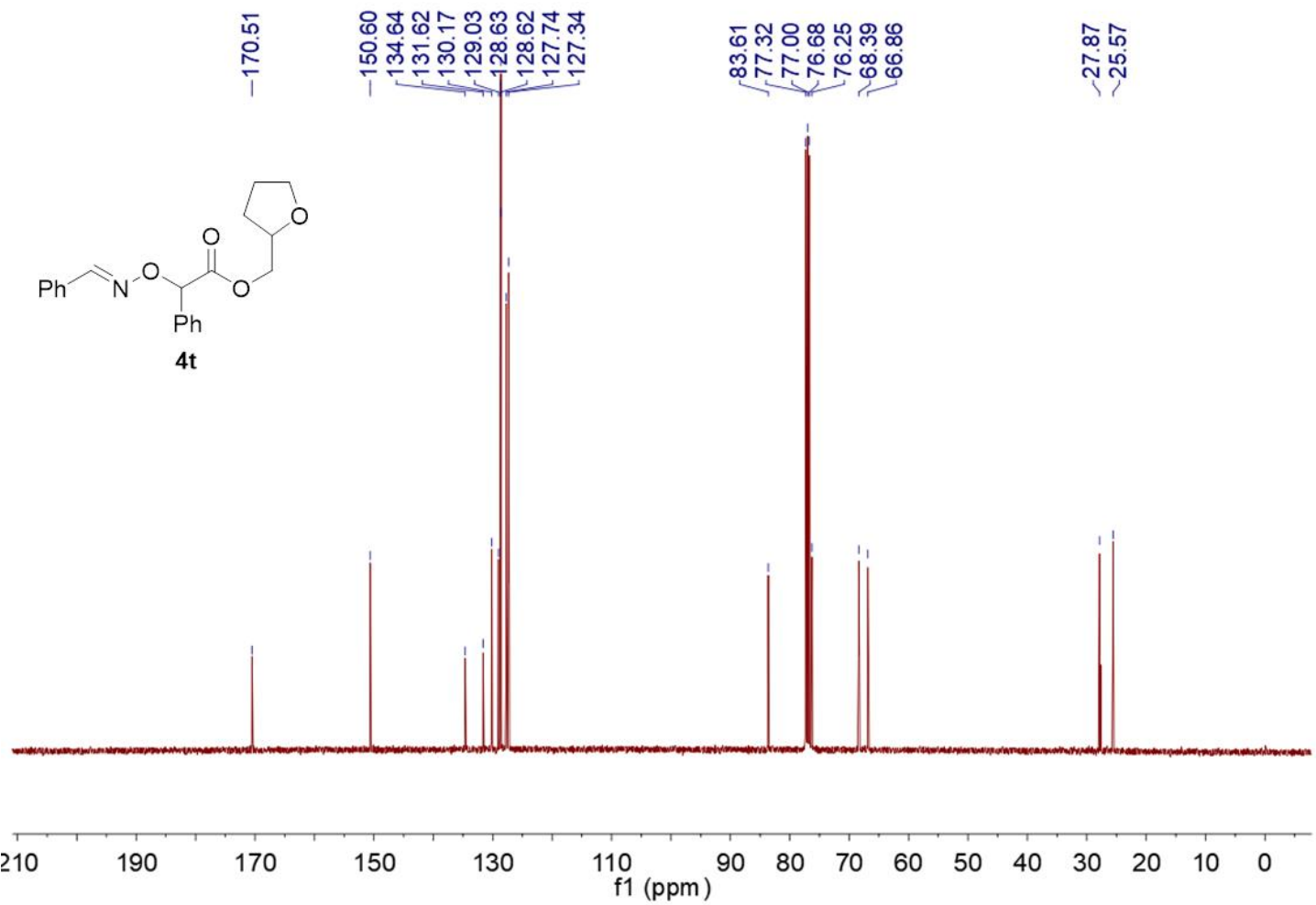


${ }^{1} \mathrm{H}$ NMR of $4 \mathbf{u}\left(400 \mathrm{MHz}, \mathrm{CDCl}_{3}\right)$

\section{ஜ

$\mathrm{Ph}_{\mathrm{Ph}} \curvearrowright \mathrm{N}_{\mathrm{O}}^{\mathrm{O}}$

$4 \mathrm{u}$

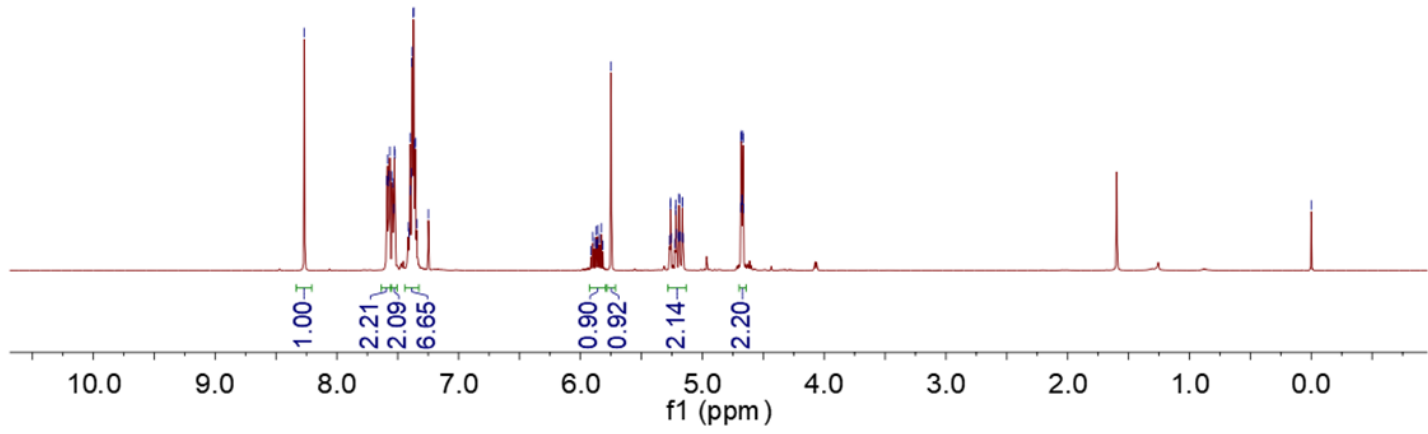

${ }^{13} \mathrm{C}$ NMR of $4 \mathbf{u}\left(100 \mathrm{MHz}, \mathrm{CDCl}_{3}\right)$
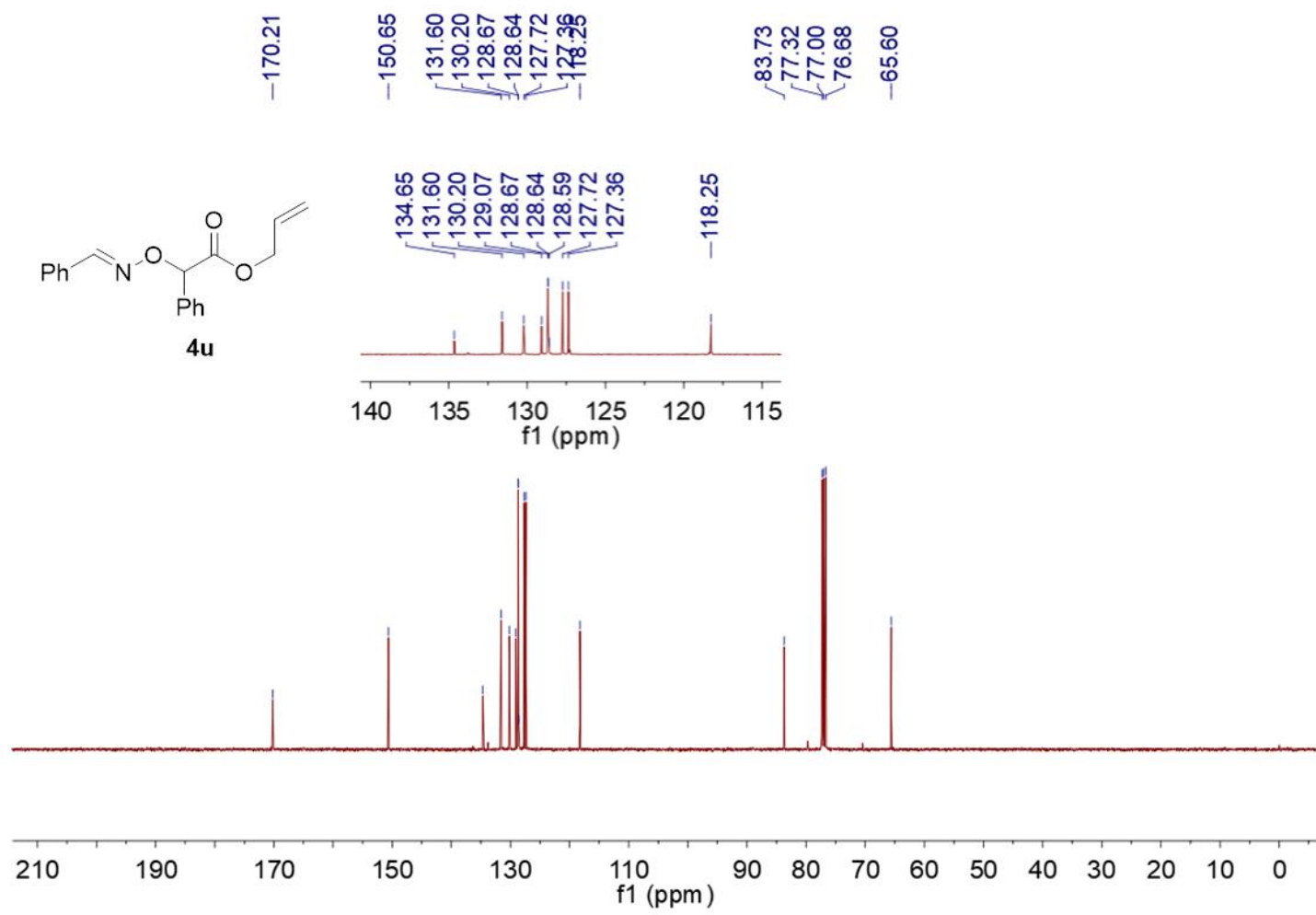
${ }^{1} \mathrm{H}$ NMR of $4 \mathbf{v}\left(400 \mathrm{MHz}, \mathrm{CDCl}_{3}\right)$
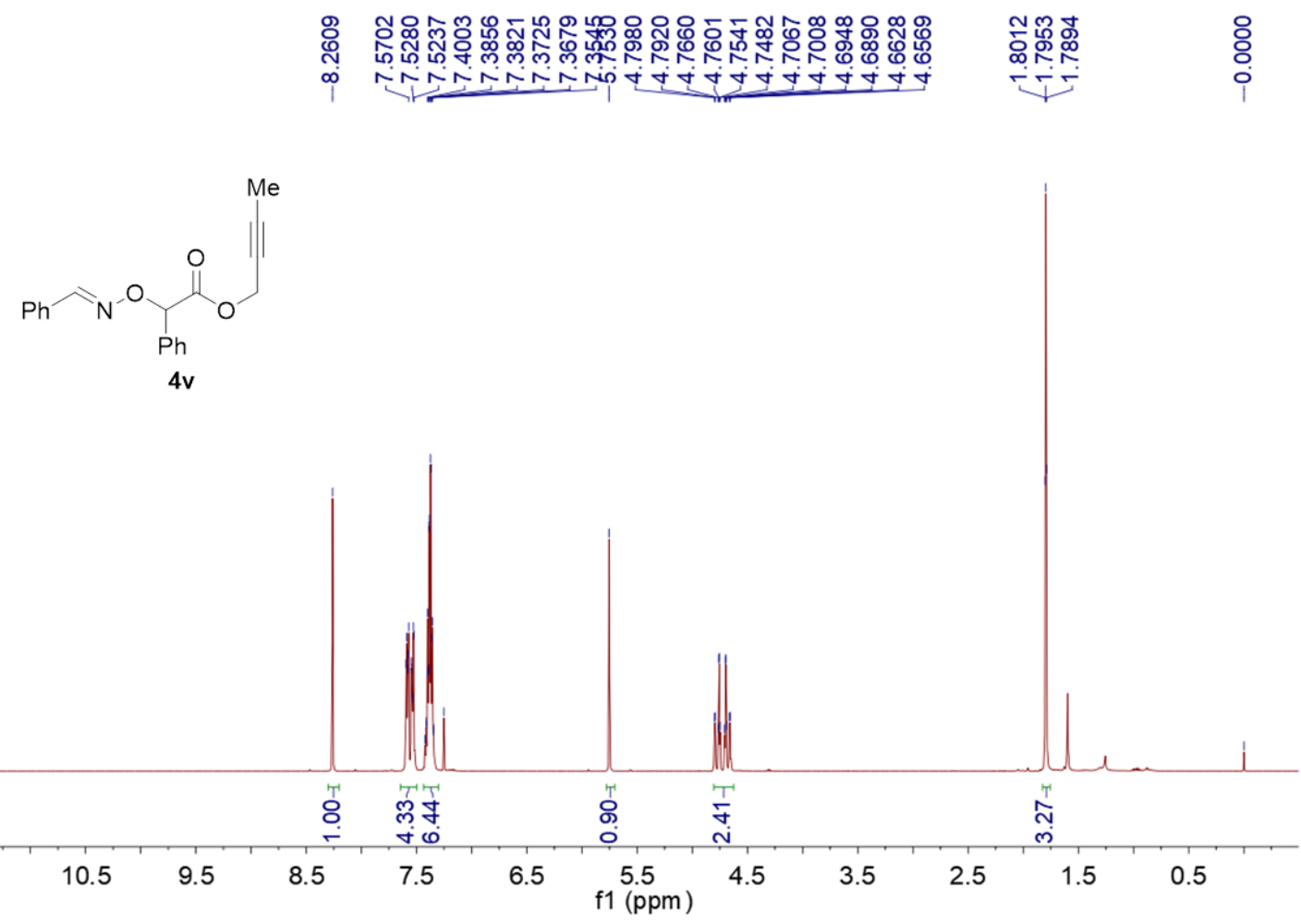

${ }^{13} \mathrm{C}$ NMR of $4 \mathbf{v}\left(100 \mathrm{MHz}, \mathrm{CDCl}_{3}\right)$
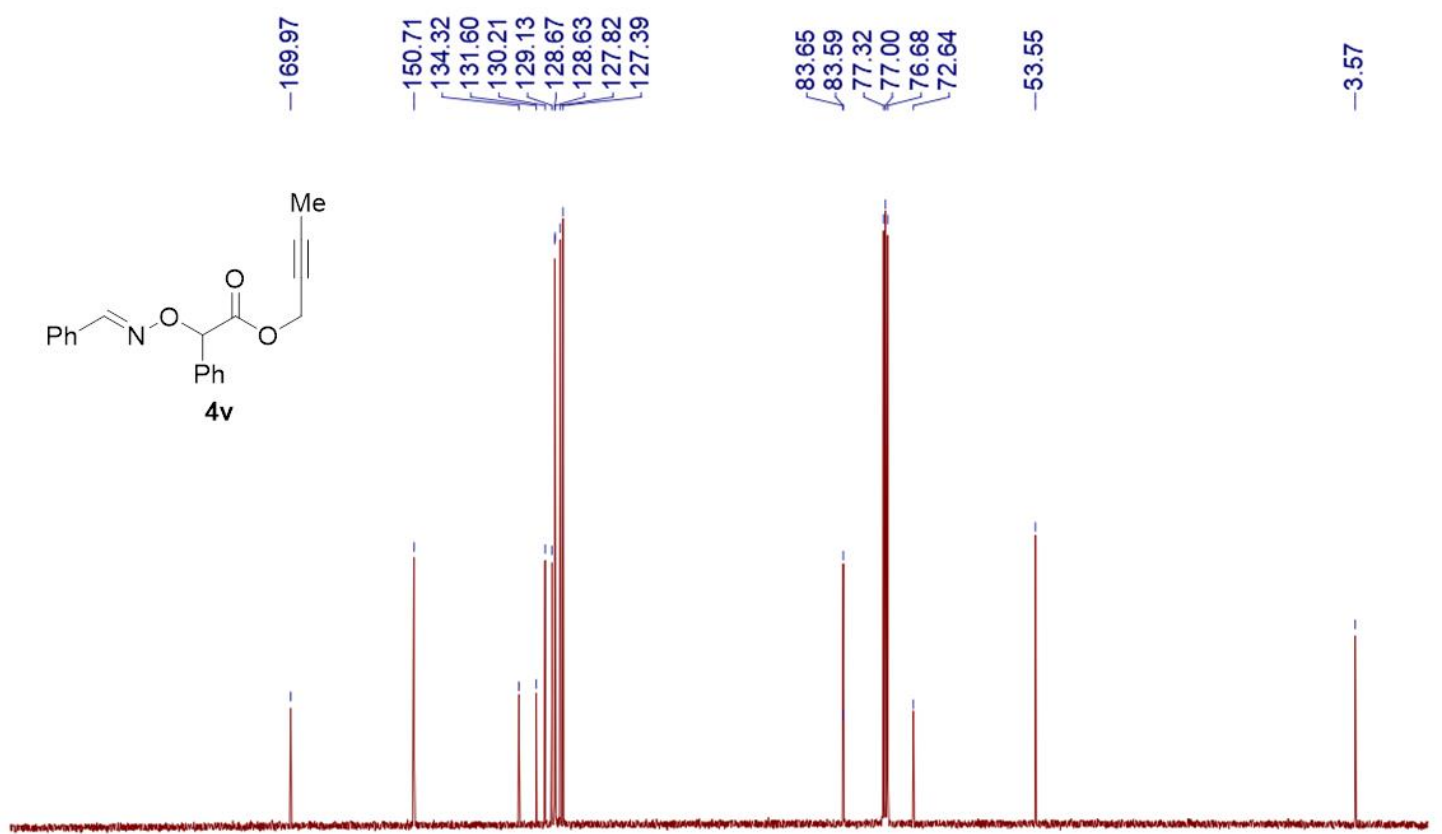

ஸึ?
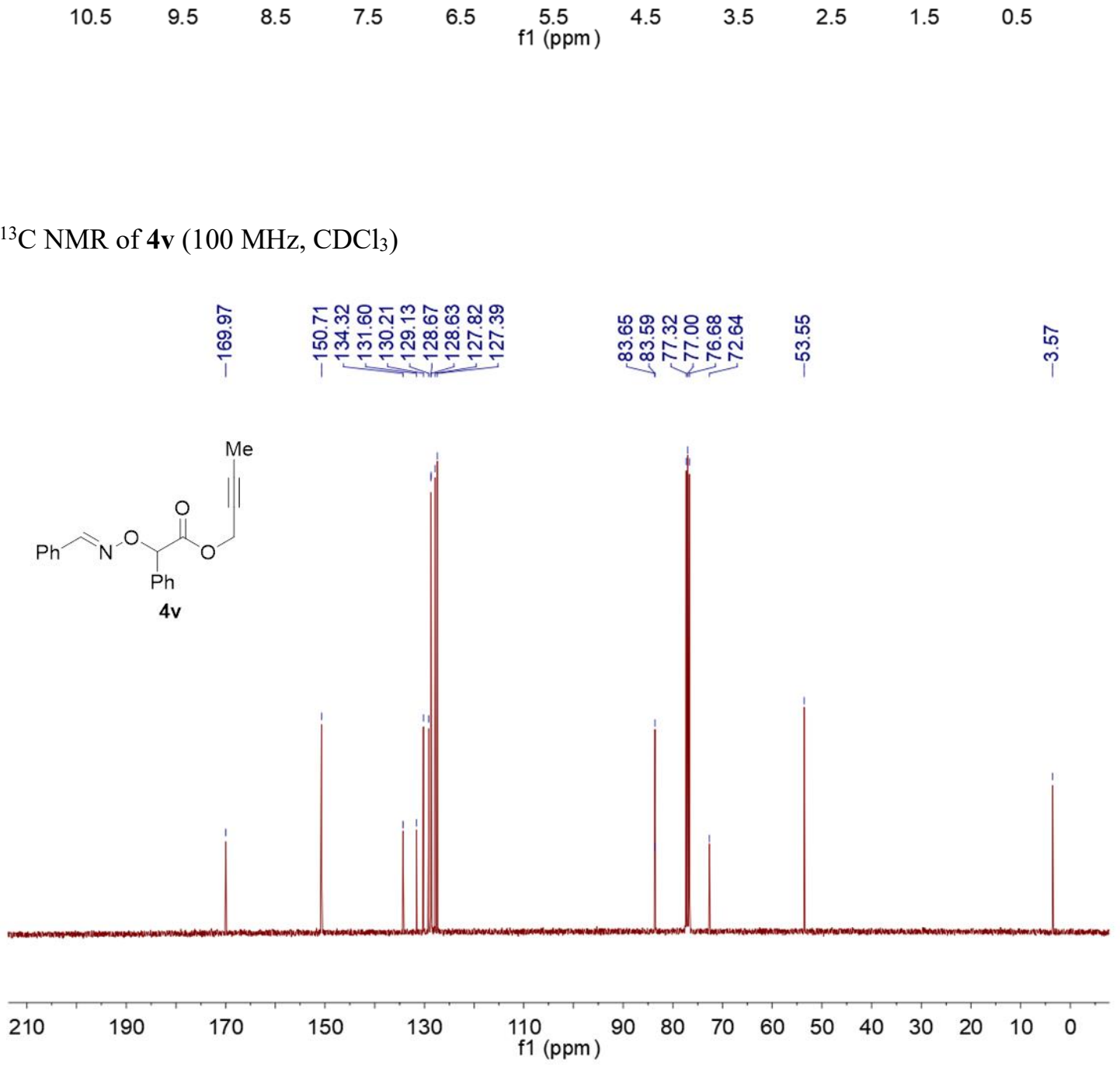
${ }^{1} \mathrm{H}$ NMR of $\mathbf{4 w}\left(400 \mathrm{MHz}, \mathrm{CDCl}_{3}\right)$

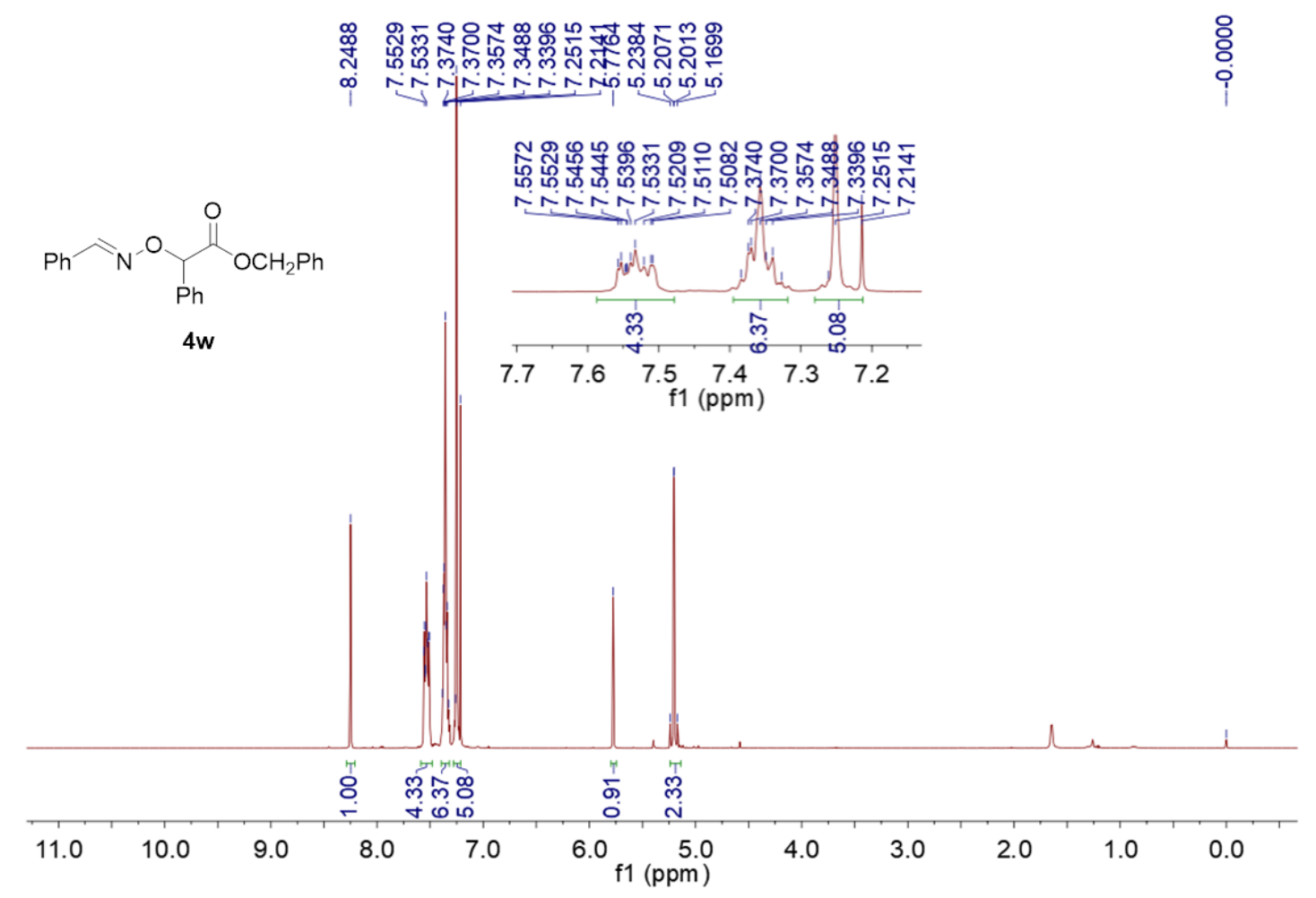

${ }^{13} \mathrm{C}$ NMR of $4 \mathbf{w}\left(100 \mathrm{MHz}, \mathrm{CDCl}_{3}\right)$

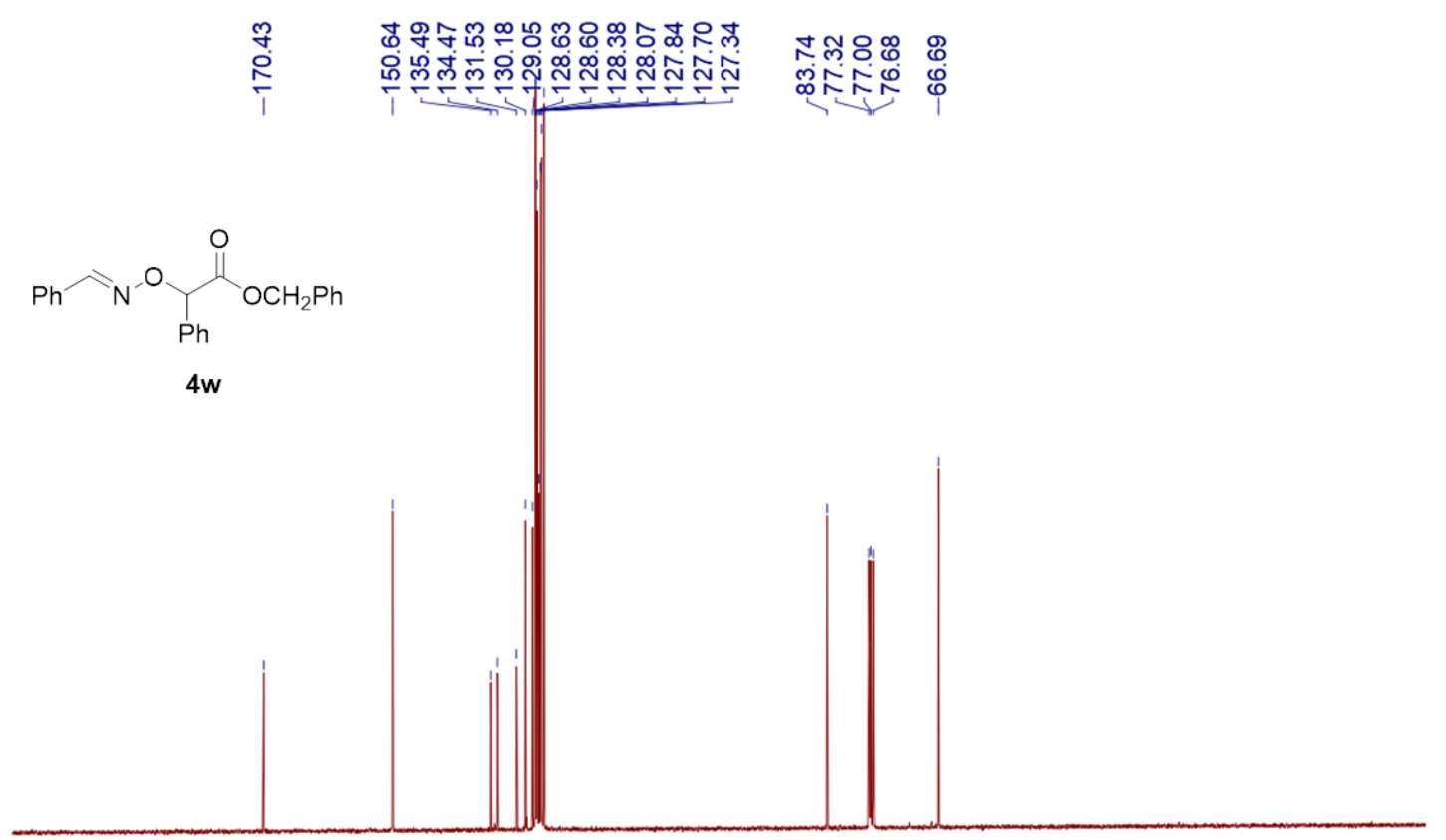

190

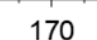

150

130

110

$90 \quad 80 \quad 70 \quad 60 \quad 50$

1 (ppm)

SI-78 
${ }^{1} \mathrm{H}$ NMR of $\mathbf{4 x}\left(400 \mathrm{MHz}, \mathrm{CDCl}_{3}\right)$

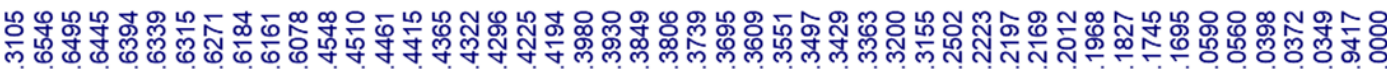

м

$\left.\mathrm{Ph}_{4 \mathbf{x}} \curvearrowright\right|_{\mathrm{Ph}} ^{\mathrm{O}} \mathrm{OPh}$

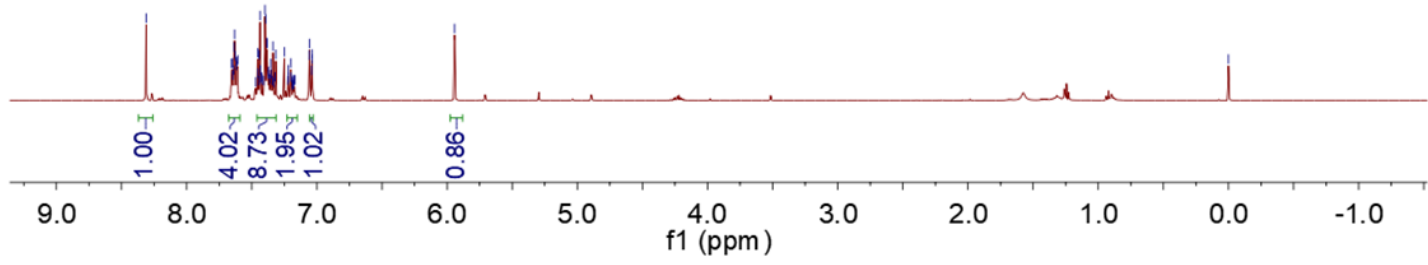

${ }^{13} \mathrm{C}$ NMR of $4 \mathbf{x}\left(100 \mathrm{MHz}, \mathrm{CDCl}_{3}\right)$

\begin{tabular}{ll}
\hline \\
0
\end{tabular}

$\mathrm{Ph}_{\mathrm{Ph}} \widehat{\mathrm{Ph}}_{\mathrm{Ph}}^{\mathrm{O}} \mathrm{H}_{\mathrm{OPh}}$

$4 x$

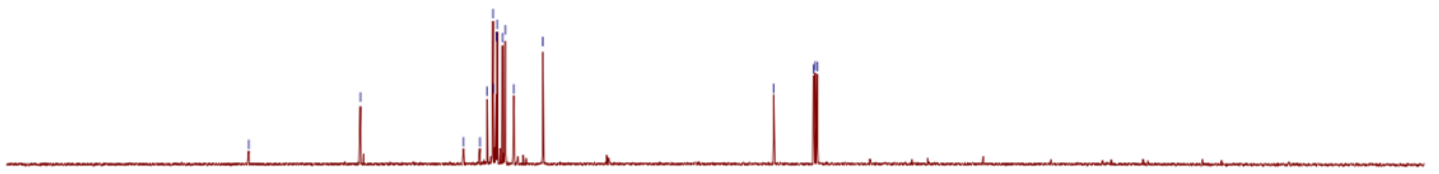

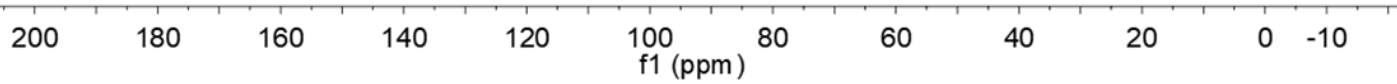


${ }^{1} \mathrm{H}$ NMR of $4 \mathbf{y}\left(400 \mathrm{MHz}, \mathrm{CDCl}_{3}\right)$

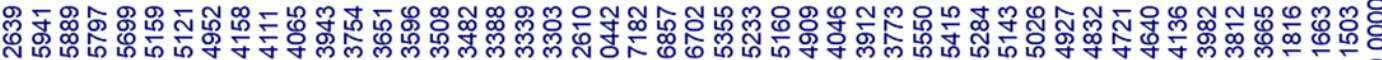

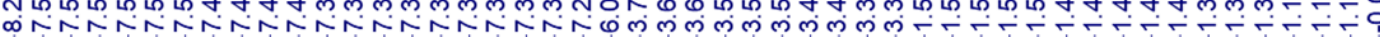<smiles>O=C(C(ON=Cc1ccccc1)c1ccccc1)N1CCCCC1</smiles>

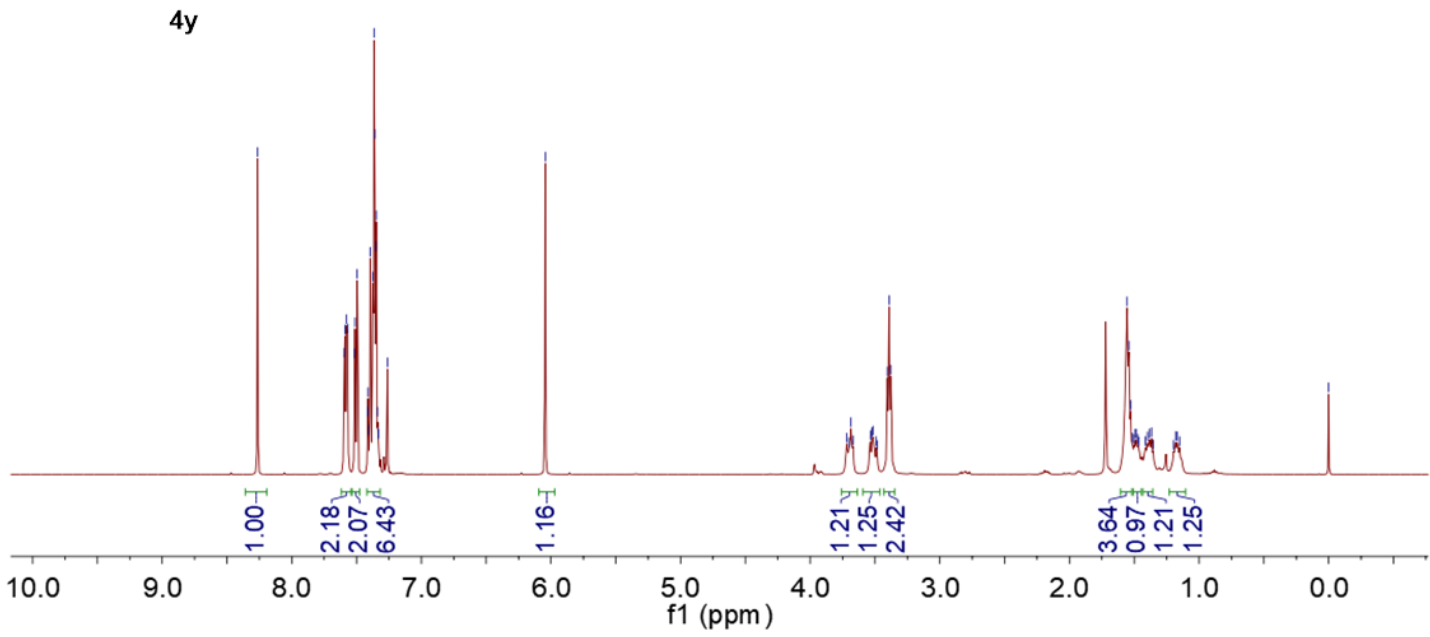

${ }^{13} \mathrm{C}$ NMR of $4 \mathbf{y}\left(100 \mathrm{MHz}, \mathrm{CDCl}_{3}\right)$
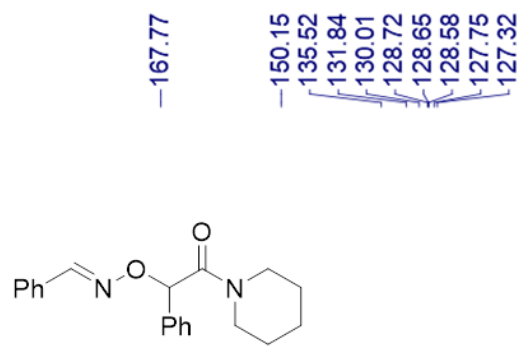

$4 y$

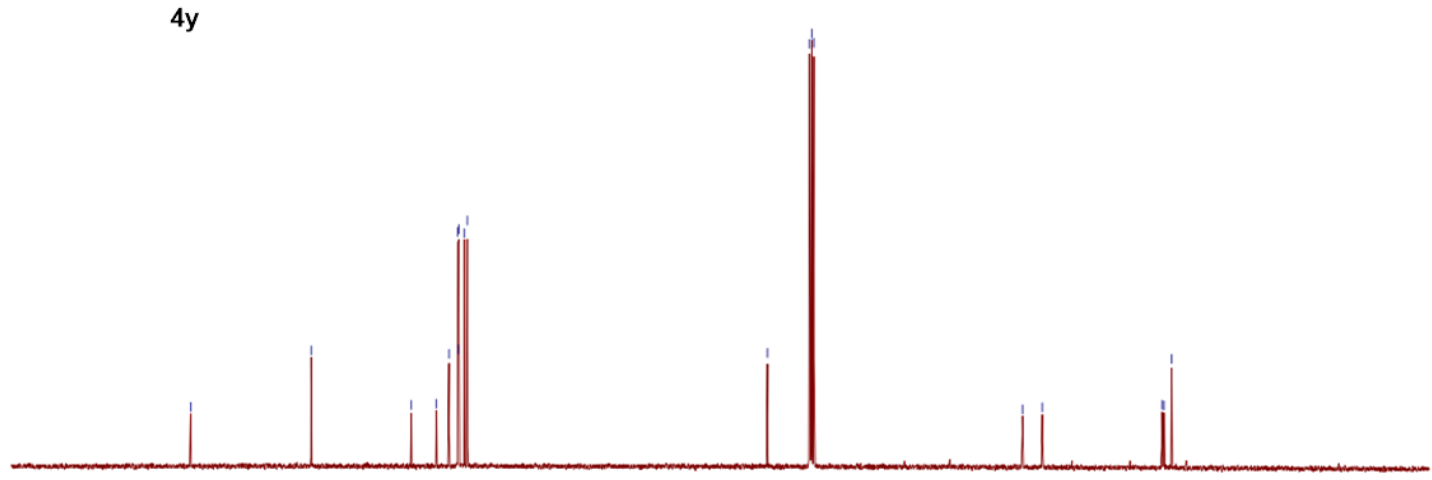

190

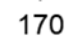

150

130

110

$90 \quad 8070$

f1 $(\mathrm{ppm})$ 
${ }^{1} \mathrm{H}$ NMR of $\mathbf{4 z}\left(400 \mathrm{MHz}, \mathrm{CDCl}_{3}\right)$

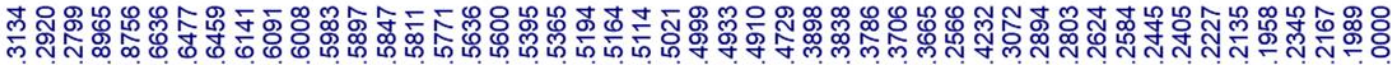

m monn

$$
\mathrm{Ph} \curvearrowright \mathrm{N}_{2 \text {-naphthyl }}^{-\mathrm{O}} \mathrm{CO}_{2} \mathrm{Et}
$$

$4 z$

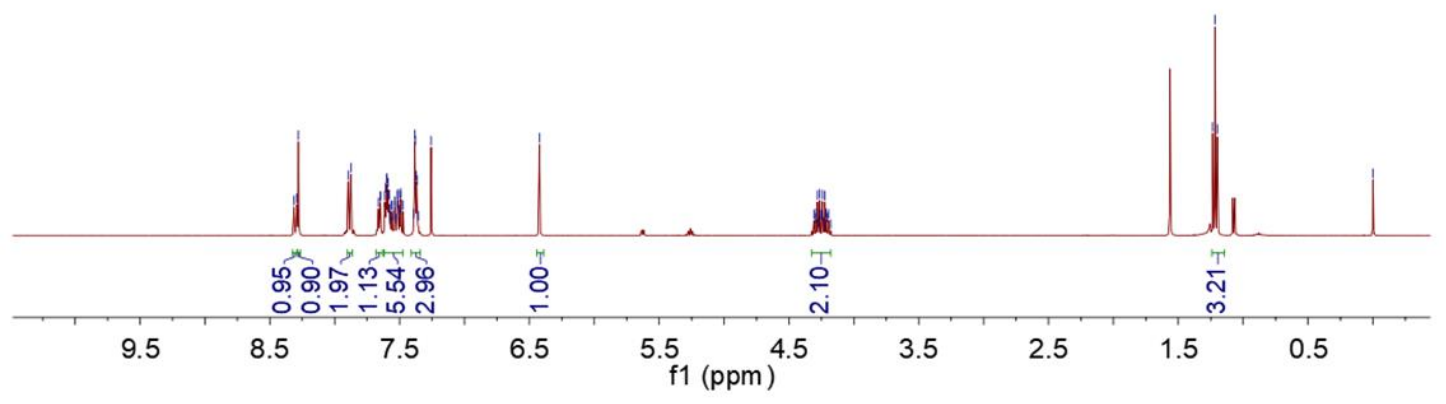

${ }^{13} \mathrm{C} \mathrm{NMR}$ of $\mathbf{4 z}\left(100 \mathrm{MHz}, \mathrm{CDCl}_{3}\right)$

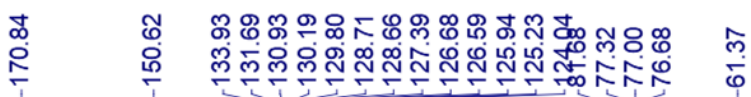

$\underset{+}{\stackrel{1}{\sim}}$

$$
\mathrm{Hz}^{2 \text {-naphthyl }}
$$

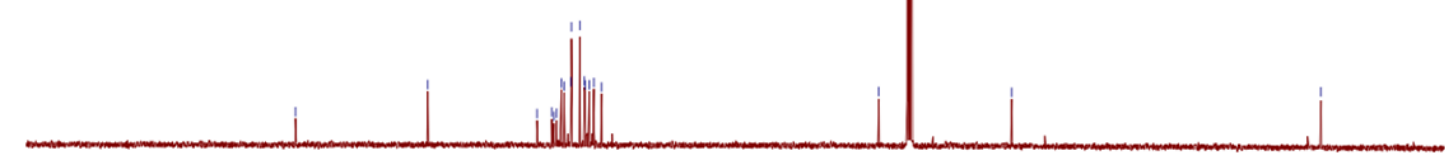

$\begin{array}{llllllllllllllllll}110 & 190 & 170 & 150 & 130 & \begin{array}{l}110 \\ \mathrm{f} 1(\mathrm{ppm})\end{array} & 90 & 80 & 70 & 60 & 50 & 40 & 30 & 20 & 10 & 0\end{array}$


11. 2D NMR spectra of 3v

DEPT 135 of $\mathbf{3 v}\left(100 \mathrm{MHz}, \mathrm{DMSO}-d_{6}\right)$

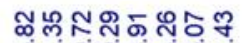

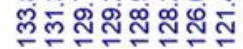

ஜ

-

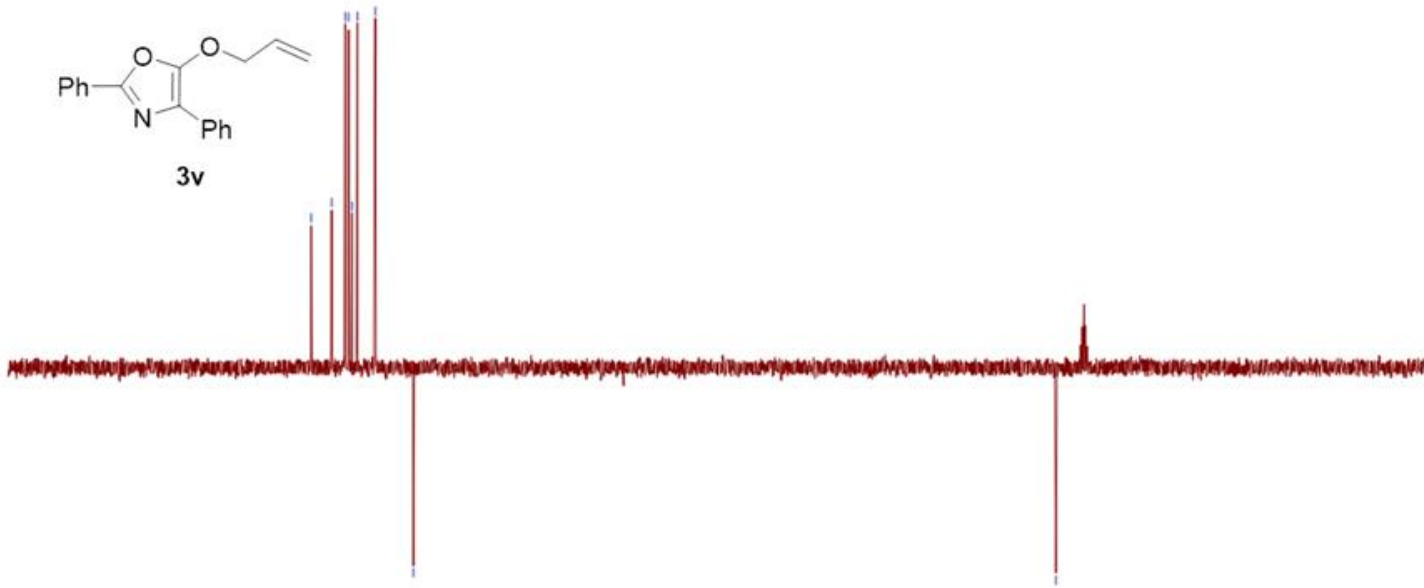

$\begin{array}{llllllllllllllllll}170 & 160 & 150 & 140 & 130 & 120 & 110 & 100 & \begin{array}{l}90 \\ \mathrm{f} 1(\mathrm{ppm})\end{array} & \begin{array}{l}80 \\ \mathrm{ppm}\end{array} & 60 & 50 & 40 & 30 & 20 & 10 & 0\end{array}$

DEPT 90 of $3 \mathbf{v}\left(100 \mathrm{MHz}\right.$, DMSO- $\left.d_{6}\right)$

กำกละกำ

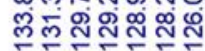

$\mathrm{Ph}-\mathrm{N}_{\mathrm{Ph}}^{\mathrm{O}}$

$3 v$

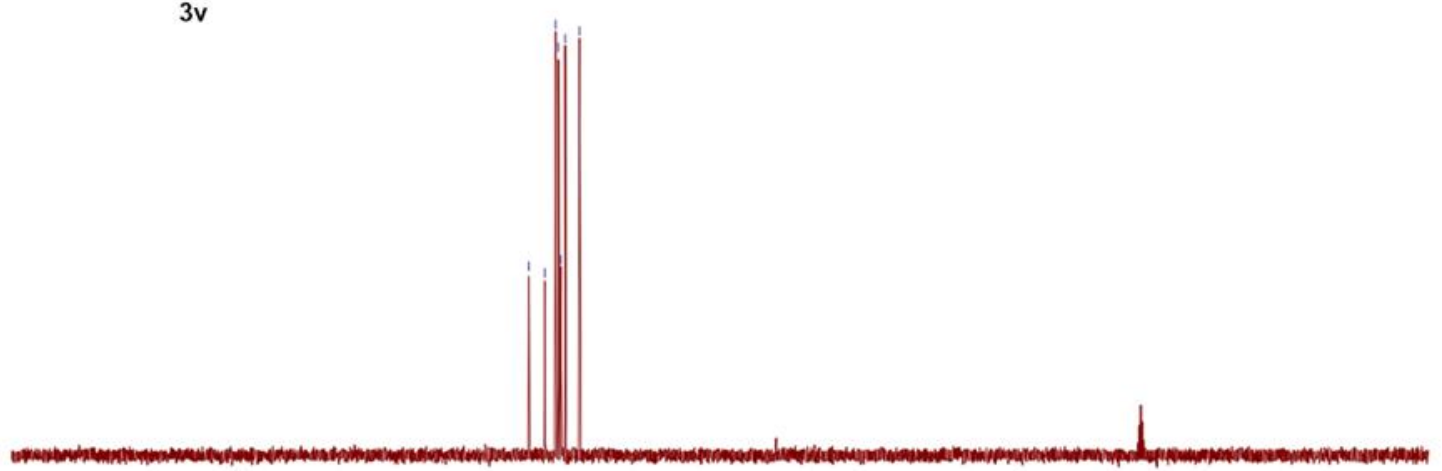

210

190

170

150

$130 \quad 110$

f1 (ppm)

$\begin{array}{llllllllll}90 & 80 & 70 & 60 & 50 & 40 & 30 & 20 & 10 & 0\end{array}$ 
COSY of $3 \mathbf{v}\left(400 \mathrm{MHz}, \mathrm{DMSO}-d_{6}\right)$

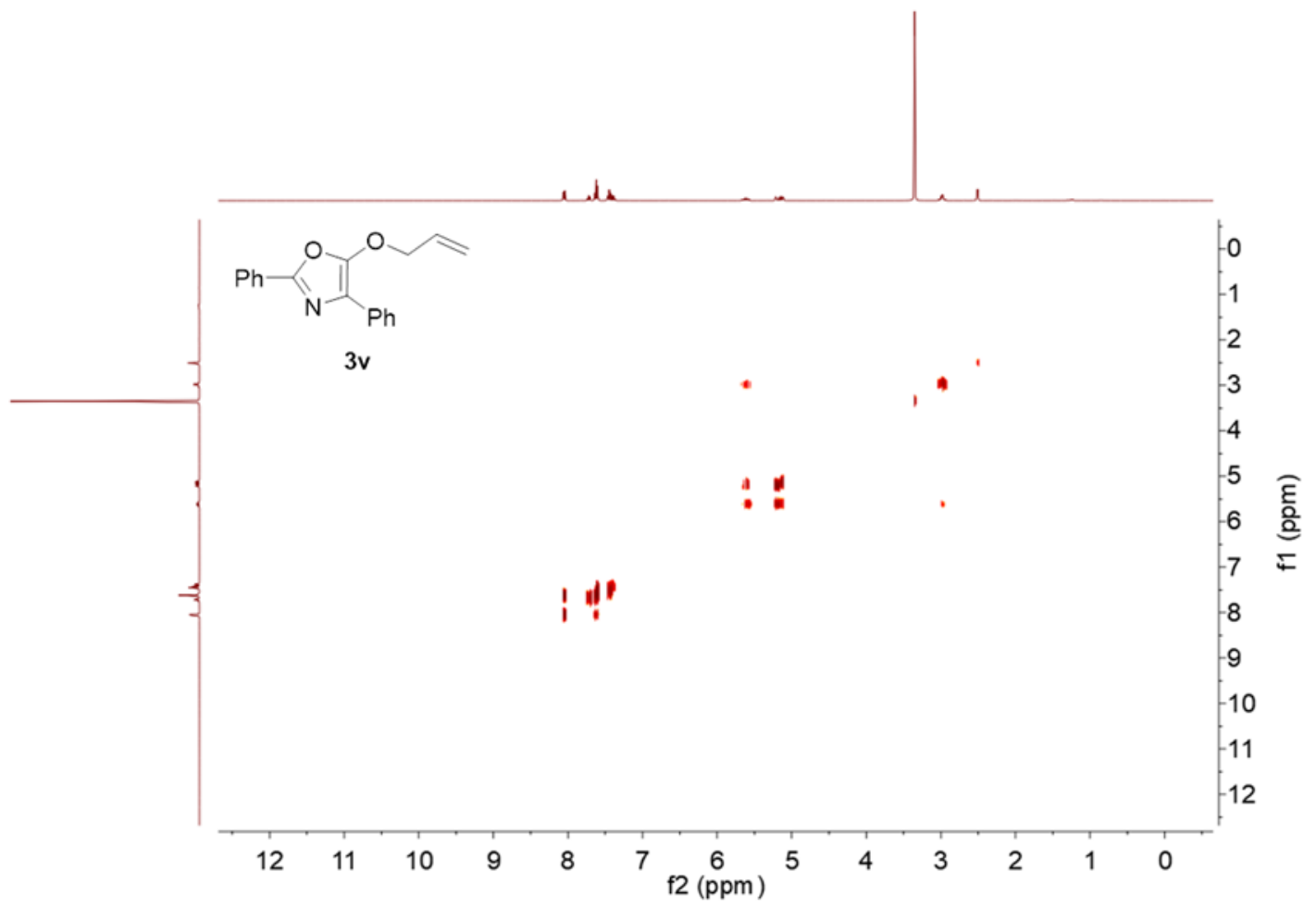

HMBC of 3v (400 MHz, DMSO- $\left.d_{6}\right)$

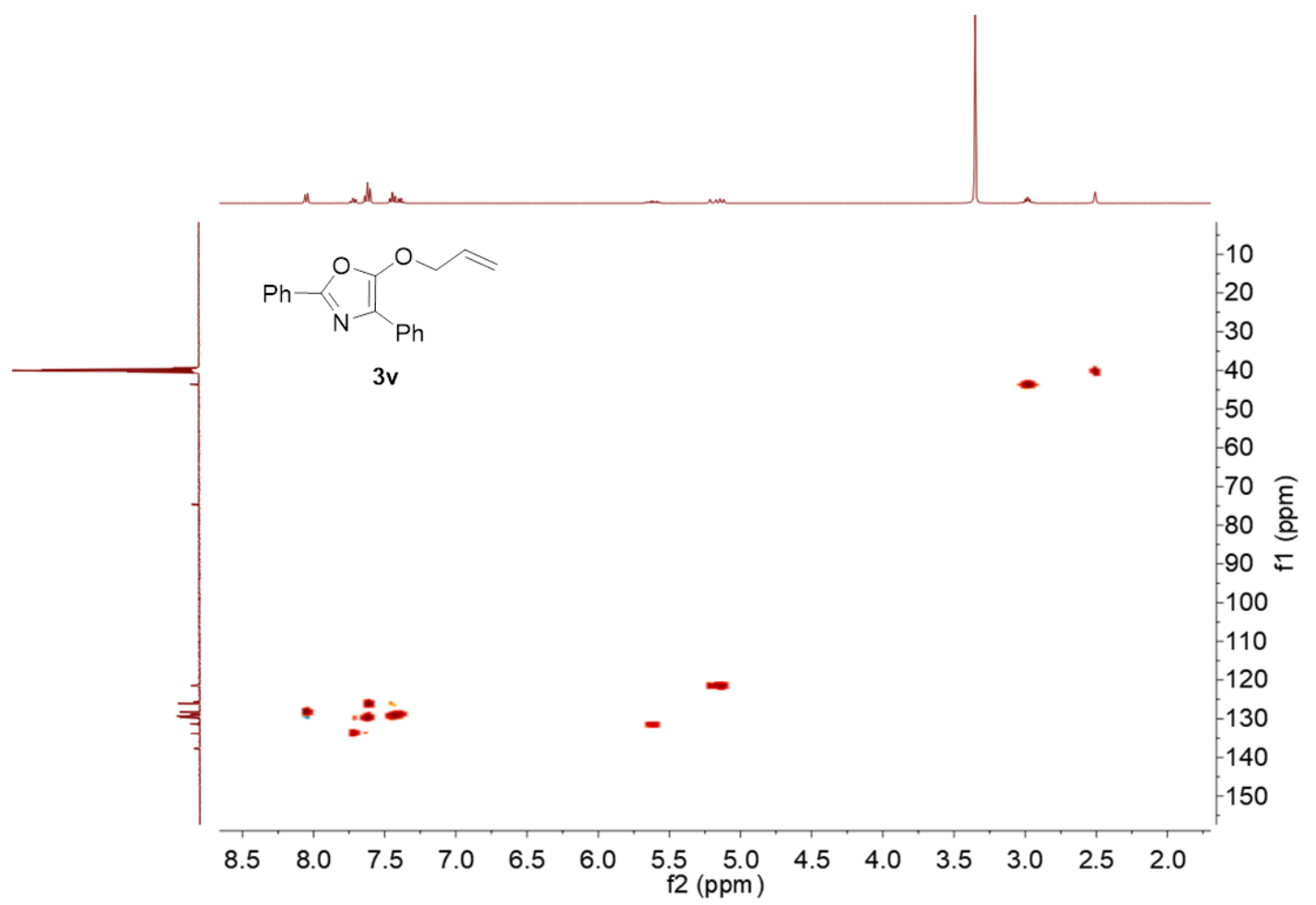


HSQC of $3 \mathbf{v}\left(400 \mathrm{MHz}, \mathrm{DMSO}-d_{6}\right)$

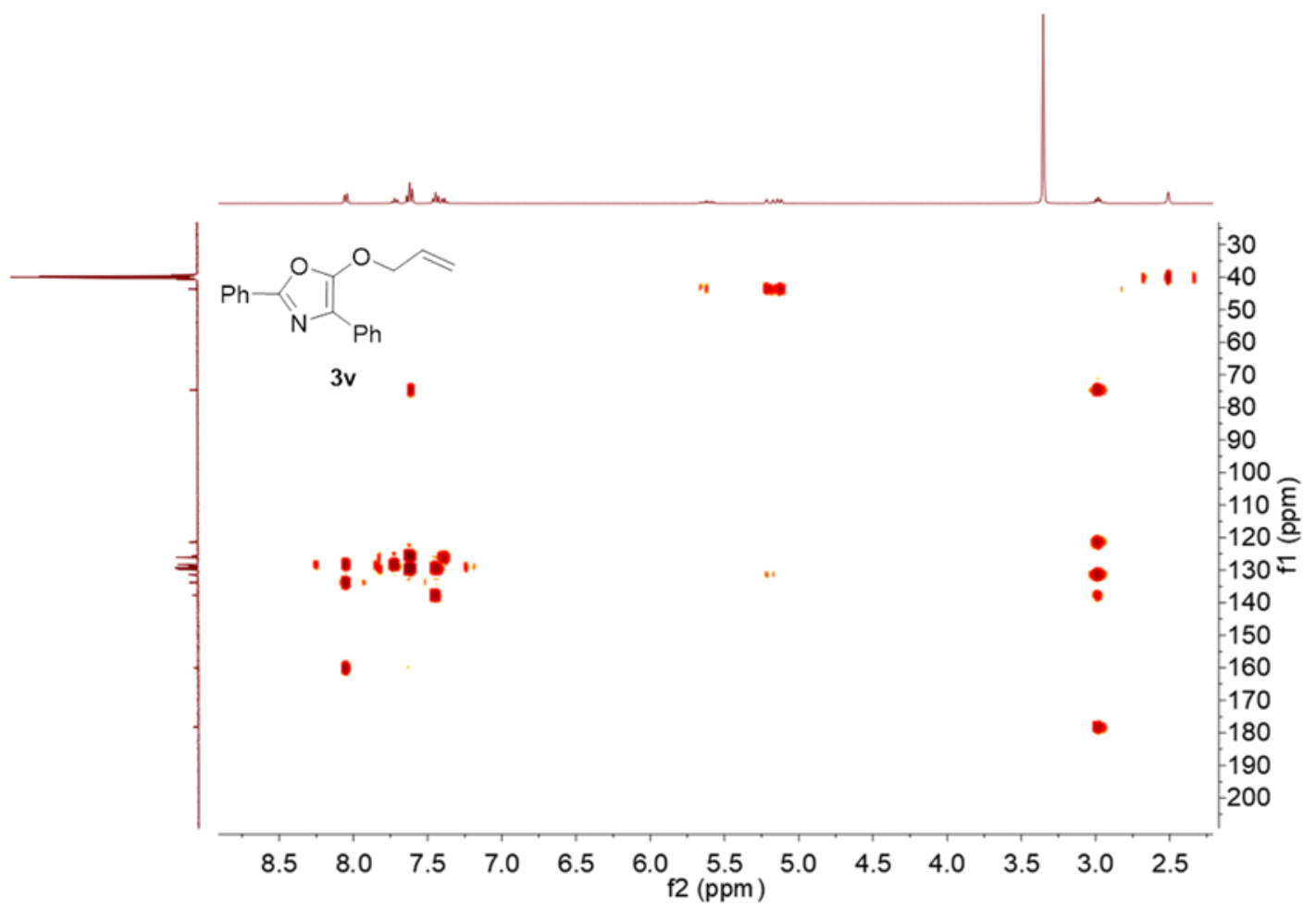

